\title{
SLIP-STACKING DYNAMICS FOR \\ HIGH-POWER PROTON BEAMS AT
}

FERMILAB

\author{
Jeffrey Eldred
}

Submitted to the faculty of the University Graduate School

in partial fulfillment of the requirements

for the degree

Doctor of Philosophy

in the Department of Physics,

Indiana University

December 2015 
Accepted by the Graduate Faculty, Indiana University, in partial fulfillment of the requirements for the degree of Doctor of Philosophy.

Doctoral Committee

Shyh-Yuan Lee, Ph.D., Committee Chair

Robert M. Zwaska, Ph.D.

Mark Messier, Ph.D.

Chen-Yu Liu, Ph.D.

Michael Berger, Ph.D.

$12 / 04 / 2015$ 
Copyright (c) 2015

Jeffrey Eldred 


\section{ACKNOWLEDGMENTS}

I will forever be indebted to my Indiana University doctoral advisor, Shyh-Yuan Lee, who believed in me and provided a foundational role in my accelerator physics education. His dedication, enthusiasm, and insight have made this work possible. I know the entire field of accelerator physics has felt the impact of his life's work and that all of us wish him the best of retirements.

Robert Zwaska, my Fermilab doctoral advisor, has been invaluable to my dissertation research and my development as a scientist. The most important part of any research effort is to identify which way to proceed and his leadership in this area is something for which I will always be grateful. Additionally, it is through his guidance that I have grown as a scientific writer and presenter.

Fermilab has felt like a place of remarkable opportunity for me and I know this is because of the hard work and collaborative spirit of the people who work there. I would particularly like to recognize Michael Backfish, Alexey Burov, Nathan Eddy, Brian Fellenz, Ioanis Kourbanis, Ao Liu, Peter Prieto, Kiyomi Seiya, Cheng-Yang Tan, Jayakar "Charles" Thangaraj, Susan Winchester, Michael Wren, and Ming-Jen Yang. I would like to give a special thanks to Phil Adamson, who played a critical role in my beam studies of slipstacking and also in my non-dissertation research on the Recycler electron cloud instability. I would also like to thank the Fermilab Main Control Room operators.

I have benefited from excellent educators, including Michael Berger and William Michael Snow, who each provided me a powerful foundation in graduate-level physics. Along 
with Michael Berger, Mark Messier and Chen-Yu Liu were kind enough to agree to be my committee members. I would also like to acknowledge Patricia Vahle, Ilya Spitkovsky, Irina Novikova, Stepan Stepanyan, and David Douglas for the research mentorship that they provided me early in my career. I would also like to personally thank the teachers of the Newport News public school system, who will always be in my heart.

I would like to thank those who have supported me by enriching my life. I feel fortunate to have many supportive friends, near and far, too numerous to name and each dear to me. I would also like to thank my siblings, Amber, Lindsey, and Tyler. You have always encouraged me. I would like to thank my parents, Christine and Stephen. Thank you for cultivating my love of learning and instilling in me my best qualities. Thank you for supporting my education. Lastly I would like to thank my beloved Emily. You are the partner I needed in this challenging time of my life. You understand me like no one else and it's wonderful that we have found each other. 


\author{
Jeffrey Eldred
}

\title{
SLIP-STACKING DYNAMICS FOR HIGH-POWER PROTON BEAMS AT
}

\section{FERMILAB}

Slip-stacking is a particle accelerator configuration used to store two particle beams with different momenta in the same ring. The two beams are longitudinally focused by two radiofrequency $(\mathrm{RF})$ cavities with a small frequency difference between them. Each beam is synchronized to one RF cavity and perturbed by the other RF cavity. Fermilab uses slip-stacking in the Recycler as to double the power of the $120 \mathrm{GeV}$ proton beam in the Main Injector.

This dissertation investigates the dynamics of slip-stacking beams analytically, numerically and experimentally. In the analytic analysis, I find the general trajectory of stable slip-stacking particles and identify the slip-stacking parametric resonances. In the numerical analysis, I characterize the stable phase-space area and model the particle losses. In particular, I evaluate the impact of upgrading the Fermilab Booster cycle-rate from $15 \mathrm{~Hz}$ to $20 \mathrm{~Hz}$ as part of the Proton Improvement Plan II (PIP-II). The experimental analysis is used to verify my approach to simulating slip-stacking loss. I design a study for measuring losses from the longitudinal single-particle dynamics of slip-stacking as a function of RF cavity voltage and RF frequency separation. 
I further propose the installation of a harmonic RF cavity and study the dynamics of this novel slip-stacking configuration. I show the harmonic RF cavity cancels out parametric resonances in slip-stacking, reduces emittance growth during slip-stacking, and dramatically enhances the stable phase-space area. The harmonic cavity is expected to reduce slip-stacking losses to far exceed PIP-II requirements. These results raise the possibility of extending slip-stacking beyond the PIP-II era.

Shyh-Yuan Lee, Ph.D., Committee Chair

Robert M. Zwaska, Ph.D.

Mark Messier, Ph.D.

Chen-Yu Liu, Ph.D.

Michael Berger, Ph.D. 


\section{CONTENTS}

1 SINGLE-RF LONGITUDINAL DYNAMICS 1

$1.1 \mathrm{RF}$ Accelerating Cavities $\ldots \ldots \ldots \ldots \ldots \ldots$

1.2 Derivation of Longitudinal Motion . . . . . . . . . . . . . . 3

1.3 Longitudinal Equations of Motion . . . . . . . . . . . . . 6

1.4 Synchrotron Oscillation Motion . . . . . . . . . . . . . 8

1.5 Slipping Particles Motion . . . . . . . . . . . . . . . 10

1.6 Acceleration \& Focusing Motion . . . . . . . . . . . . . . . . . . . 11

1.7 Summary . . . . . . . . . . . . . . . . . . . . . 14

2 FERMILAB ACCELERATOR COMPLEX 15

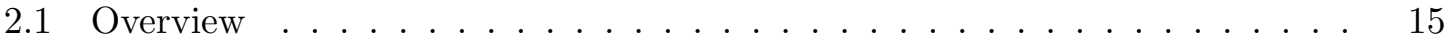

2.2 Injector \& $\operatorname{Linac} \ldots \ldots \ldots \ldots \ldots$

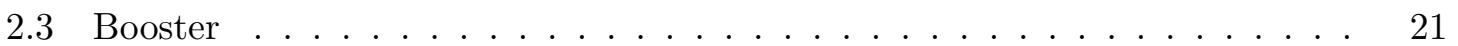

2.4 Recycler \& Main Injector . . . . . . . . . . . . . . . . 26

2.5 PIP-II \& PIP-III . . . . . . . . . . . . . . . . . . . . . . . . . . 32

3 SLIP-STACKING DYNAMICS $\quad 36$

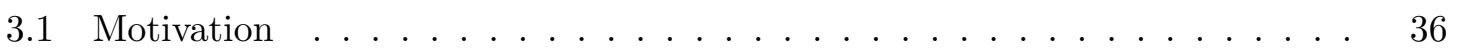

3.2 Slip-stacking Accumulation Scheme . . . . . . . . . . . . . . 38

3.3 Independent Buckets Approximation . . . . . . . . . . . . . . . . 40 
3.4 General Perturbative Solution . . . . . . . . . . . . . . . . . . 42

3.5 Stability Maps and Area Factors . . . . . . . . . . . . . . . . 46

3.6 Injection Efficiency of Gaussian Beams . . . . . . . . . . . . . . . 51

3.7 Recycler Slip-stacking Constraints _. . . . . . . . . . . . . . 56

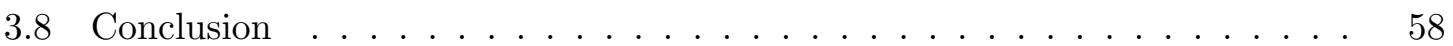

4 SLIP-STACKING LOSS MEASUREMENTS 60

4.1 Tomography Measurements of Beam Distribution . . . . . . . . . . . . . 60

4.2 Overview of Slip-stacking Beam Loss Study . . . . . . . . . . . . . . . 61

4.3 Loss Study Results . . . . . . . . . . . . . . . . . . . . . . 64

4.4 Simulation of Loss Study . . . . . . . . . . . . . . . . . . 70

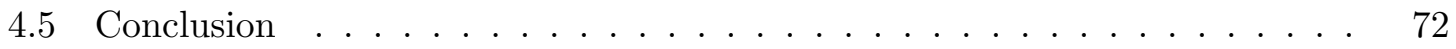

5 HARMONIC SLIP-STACKING DYNAMICS

5.1 Motivation . . . . . . . . . . . . . . . . 75

5.2 Slip-stacking with Harmonic RF f . . . . . . . . . . . . . . 76

5.3 Stability Maps and Area Factors _. . . . . . . . . . . . . . . 78

5.4 Poincaré Maps . . . . . . . . . . . . . . . . . . . . . . . . . 83

5.5 Injection Efficiency of Gaussian Beams . . . . . . . . . . . . . . . . . . 84

5.6 Mitigation of Emittance Growth _ . . . . . . . . . . . . . 89

5.7 Chapter Conclusion . . . . . . . . . . . . . . . . . 94

5.8 Dissertation Conclusion _. . . . . . . . . . . . . . . . . . . 94

$\begin{array}{ll}\text { Appendices } & 96\end{array}$

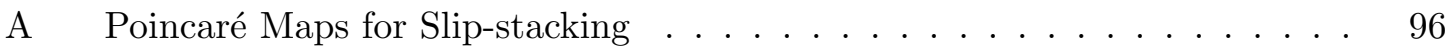

B Slip-stacking Perturbative Solution _. . . . . . . . . . . 106

C Stability Maps for Slip-stacking . . . . . . . . . . . . . . . . 113 
D Stability Maps for Harmonic Slip-stacking _ . . . . . . . . . . . . 123

E Poincaré Maps for Harmonic Slip-stacking _ . . . . . . . . . . . . . 133

Bibliography

CV 


\section{CHAPTER 1}

\section{Single-RF Longitudinal Dynamics}

In this chapter, we provide an introduction to longitudinal dynamics in particle accelerators. Slip-stacking is a complex problem in longitudinal dynamics that is best understood as a perturbation on the single-RF system that is presented in this chapter.

\subsection{RF Accelerating Cavities}

Particle beams are accelerated in high-energy machines by devices known as radiofrequency (RF) accelerating cavities. RF cavities are resonant devices used to trap an oscillating electromagnetic field which acts on the charged particle beam via the Lorentz force. RF cavities trap electromagnetic waves only at discrete frequencies (eigenfrequencies), with corresponding spatiotemporal distributions (eigenmodes). Typically, RF cavities are only operated at the lowest order mode, known as the fundamental or $\mathrm{TM}_{010}$ mode, with higher order modes being unwanted deviations from simple longitudinal acceleration. We will use the term RF frequency $\omega_{r f}=2 \pi f_{r f}$ to refer specifically to the accelerating mode.

Fig. 1.1 shows a simple RF cavity operating in the $\mathrm{TM}_{010}$ mode. The electric field is parallel (or antiparallel) to the motion of the beam and the magnetic field is perpendicular and rotationally symmetric. In a purely cylindrical cavity, the RF frequency is determined by the geometry of the cylinder. However the ferrite tuner is electromagnetic coupled to the body of the cavity and it can be used to adjust the RF frequency of the cavity. A 
bias current is applied through the ferrite tuner which adjusts the magnetic permeability of the ferrite rings and consequently shifts the RF frequency as needed. Other RF cavities change the resonant frequency with mechanical tuners. The electromagnetic waves generate heat due to the surface resistance of the RF cavity and this heat is compensated by cooling elements that are not shown in Fig. 1.1.

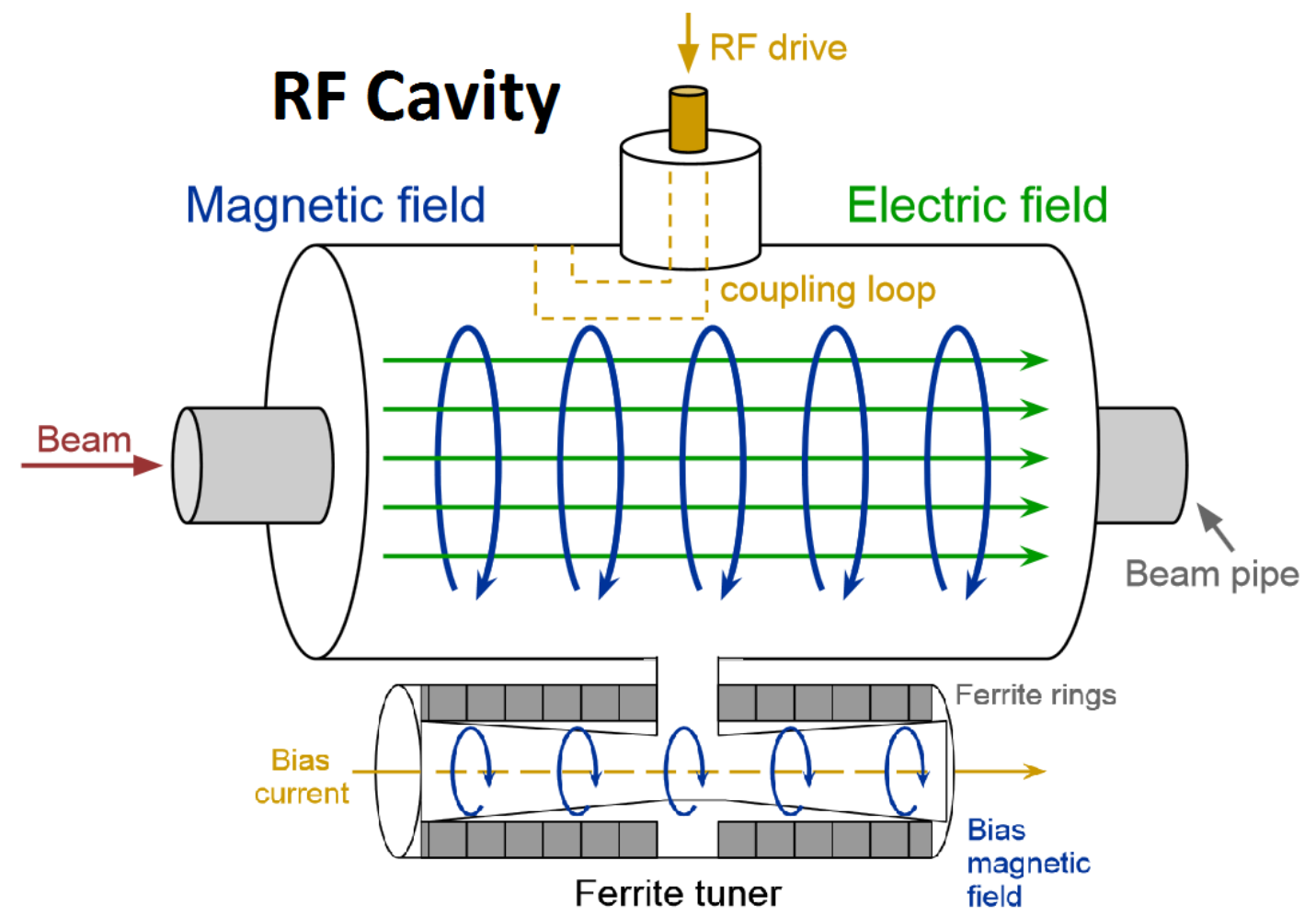

Figure 1.1: A diagram of a simplified RF cavity. [1]

High-voltage RF electronics are used to amplify a sinusoidal signal and drive the RF cavity. From an electronic perspective, the RF cavity can be approximated by the RLC resonant circuit shown in Fig. 1.2. The corresponding resonance frequency is given by

$$
f_{r f}=\frac{1}{2 \pi \sqrt{L_{e q} C_{e q}}}
$$

where $L_{e q}$ is equivalent inductance and $C_{e q}$ is the equivalent capacitance. Another important parameter is the quality factor of the cavity, known as $Q$, which is the ratio of energy stored 
to energy dissipated per cycle. From the circuit model, the Q-factor is given by:

$$
Q=R_{s h} \frac{C_{e q}}{L_{e q}}
$$

where $R_{s h}$ is the shunt impedance. As shown in Fig. 1.2, a higher $Q$-factor corresponds to a sharper the frequency resonance.
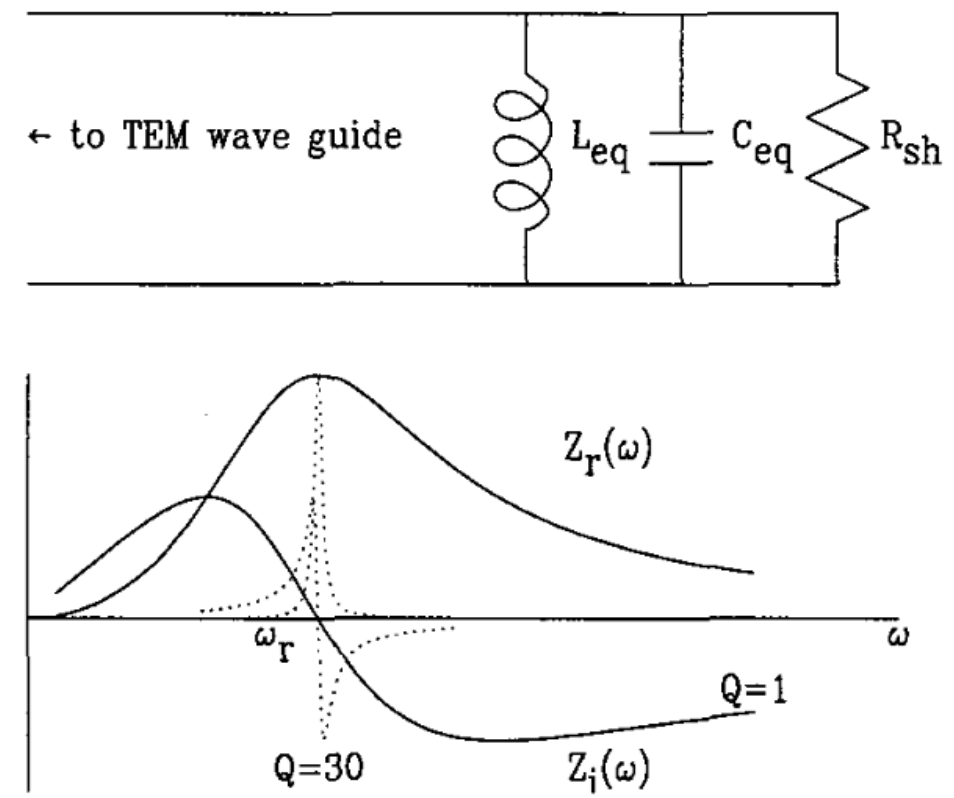

Figure 1.2: (top) An equivalent RLC circuit corresponding to a resonant RF cavity. (bottom) The complex impedance of the RLC circuit for $Q=1$ and $Q=30$. [2]

\subsection{Derivation of Longitudinal Motion}

Here we derive the longitudinal motion of charged particles accelerated by an RF cavity. For now, we consider only the single-particle motion and then we relate our results to the ensemble of particles that make up a charged particle beam. The longitudinal coordinates of a charged particle are a position coordinate $\phi$ and a momentum coordinate $\delta$. The $\phi$ coordinate is the arrival time of a charged particle relative to the oscillation of RF wave in the cavity. The $\delta$ coordinate is the fractional deviation of the particle momentum from a 
reference momentum.

The acceleration of a charged particle passing through a time-varying accelerating field is given by

$$
\Delta E=q V \frac{\beta c}{d} \int_{-d / 2 \beta c}^{d / 2 \beta c} \sin \left(\omega_{r f} t+\phi\right) d t
$$

where $q$ is the charge of particle, $V$ is the RF cavity voltage, $\beta c$ is the velocity of the particle, $d$ is the length of the accelerating gap in the RF cavity, and $\phi$ is the phase corresponding to the arrival time of the incoming particle. The integral given in Eq. 1.3 is trivial to solve:

$$
\Delta E=q V(\sin \chi / \chi) \sin (\phi)
$$

where $\chi=\omega_{r f} d / 2 \beta c$. The term $\sin \chi / \chi$ is referred to as the transit time factor and hereafter we use the effective cavity voltage $V(\sin \chi / \chi) \rightarrow V$.

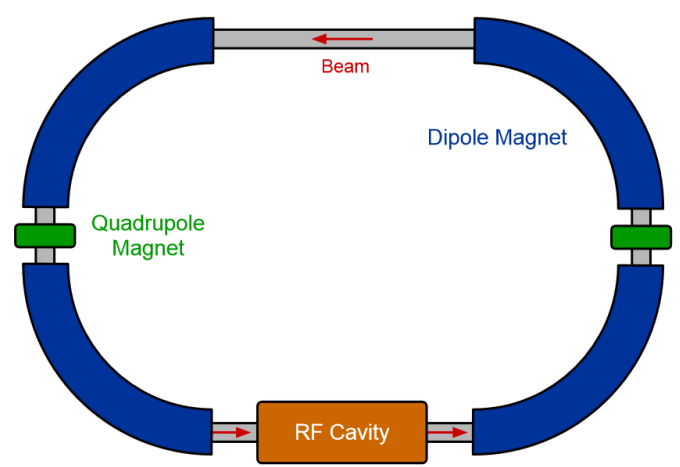

Figure 1.3: A simplified particle accelerator ring. The dipole magnets curve the beam so that it can be accelerated many times by the same RF cavity. The quadrupole magnets transversely focus the beam so it does not diverge into the beampipe wall as it is transported. [1]

In a cyclic particle accelerator a particle beam is directed along a closed curve and passes through the same sequence of beamline elements each revolution. Fig. 1.3 shows a diagram of a simple ring, where dipoles are used to bend the beam in a loop, quadrupoles are used to focus the beam transversely, and an RF cavity is used to accelerate the beam. In order 
for an RF cavity to act coherently on a particle over many passes, the RF frequency $f_{r f}$ of the particle must be near a harmonic multiple $h$ of the revolution frequency $f_{\text {rev }}$. When $f_{r f}=h f_{r e v}$ we refer to this as the reference frequency and the corresponding momentum of the particle as the synchronous momentum $p_{0}$. We define our momentum coordinate $\delta$ as the fractional deviation from the synchronous momentum:

$$
\delta \equiv \frac{p-p_{0}}{p_{0}}
$$

Using Eq. 1.4 and Eq. 1.5 we write the change in the delta coordinate per unit time.

$$
\begin{aligned}
& \dot{\delta}=\frac{\dot{p}}{p_{0}}=\frac{\dot{E}}{\beta^{2} E_{0}}=f_{\text {rev }} \frac{\Delta E}{\beta^{2} E_{0}}=f_{\text {rev }} \frac{q V}{\beta^{2} E_{0}} \sin (\phi) \\
& \dot{\delta}=f_{\text {rev }} V_{\delta} \sin (\phi)
\end{aligned}
$$

where $V_{\delta}=\frac{q V}{\beta^{2} E_{0}}$ is the maximum fractional change in the reference momentum within a single revolution.

The revolution period depends on the particle momentum and determines the change in the phase $\phi$ during each revolution. The phase-slip factor $\eta$ is defined to be the linear dependence of the revolution period on particle momentum:

$$
\frac{T-T_{r e v}}{T_{r e v}} \approx 0+\frac{1}{T_{r e v}} \frac{\partial T}{\partial \delta} \delta=\eta \delta
$$

Recall that we've defined the reference momentum such that $T=T_{\text {rev }}$ when $\delta=0$. Particles at greater momentum take less time to travel the same path length but generally take longer path lengths through particle accelerators. Consequently, the phase-slip factor $\eta$ can be positive or negative but will generally increase with energy:

$$
\eta=\frac{1}{T_{r e v}} \frac{\partial T}{\partial \delta}=\frac{1}{C} \frac{\partial C}{\partial \delta}-\frac{1}{\beta} \frac{\partial \beta}{\partial \delta}=\frac{1}{\gamma_{T}^{2}}-\frac{1}{\gamma^{2}}
$$

where $C$ is the path length of the particle beam in one revolution and is referred to as the circumference. The parameter $\gamma_{T}$ is calculated from the properties of the accelerator lattice 
and corresponds to the energy at which the phase-slip factor is zero. A synchrotron is a cyclic particle accelerator that is designed to operate with a nonzero phase-slip factor. For a non-accelerating beam we take $\eta$ to be a given constant parameter.

Using Eq. 1.7 and taking $\eta$ to be constant, we can write the change in the $\phi$-coordinate as a function of the $\delta$-coordinate:

$$
\begin{aligned}
& \dot{\phi}=f_{r e v} \Delta \phi=2 \pi f_{r e v} \frac{\Delta T}{T_{r f}}=2 \pi f_{r e v} h \frac{\Delta T}{T_{r e v}} \\
& \dot{\phi}=2 \pi f_{r e v} h \eta \delta
\end{aligned}
$$

\subsection{Longitudinal Equations of Motion}

Taking Eq. 1.6 and Eq. 1.9 together, we find the complete equations of motion in the form of coupled first-order differential equations:

$$
\dot{\delta}=f_{r e v} V_{\delta} \sin (\phi), \dot{\phi}=2 \pi f_{r e v} h \eta \delta
$$

The corresponding second-order equation of motion is

$$
\ddot{\phi}=-\omega_{s}^{2} \sin (\phi)
$$

where

$$
\omega_{s}=2 \pi f_{r e v} \sqrt{\frac{V_{\delta} h|\eta|}{2 \pi}}
$$

The frequency of small oscillations $\omega_{s}$ is referred to as the synchrotron frequency. We have adopted the convention that if $\eta>0$ then $\phi \rightarrow \phi+\pi$ so that the stable fixed point is always obtained at $\phi=0$ and the unstable fixed point is always found at $\phi=\pi$. Clearly Eq. 1.11 describes a system isomorphic to the simple pendulum. Fig. 1.4 shows the trajectories of particles governed by Eq. 1.10.

For small $\phi$, Eq. 1.11 has a stable solution known as a synchrotron oscillation

$$
\phi=\rho \sin \left(\omega_{s} t+\psi\right)
$$




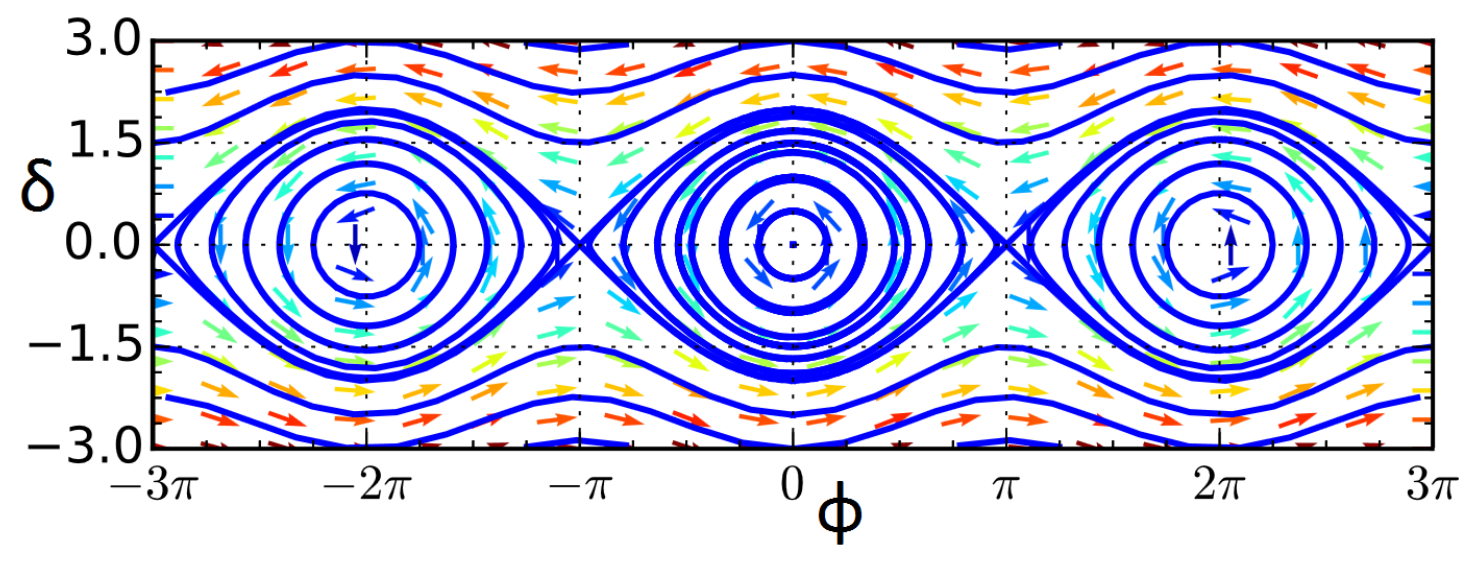

Figure 1.4: Several phase-space trajectories for the synchrotron motion (blue lines) alongside the instantaneous phase-space motion (arrows). Here, $\eta<0$.

where the amplitude $\rho$ and the initial phase $\psi$ are set by initial conditions.

The Hamiltonian corresponding to Eq. 1.10 is given by

$$
H=\pi f_{r e v} h|\eta| \delta^{2}+f_{r e v} V_{\delta}[1-\cos (\phi)]
$$

The sign of Eq. 1.14 has been fixed (for positive and negative $\eta$ ) so that $H$ is nonnegative and is zero only at the stable fixed point. The separatrix $\left(H=2 f_{r e v} V_{\delta}\right)$ is given by

$$
\delta= \pm \sqrt{\frac{V_{\delta}}{\pi h|\eta|}} \sqrt{1+\cos (\phi)}= \pm \frac{2}{h|\eta|} \frac{\omega_{s}}{\omega_{\text {rev }}} \cos \left(\frac{\phi}{2}\right)
$$

Taking the integral over the separatrix, the total stable phase-space area can be calculated $(\phi \cdot \delta$ units $):$

$$
\mathcal{A}_{0}=2 \int_{-\pi}^{\pi} \frac{2}{h|\eta|} \frac{\omega_{s}}{\omega_{\text {rev }}} \cos \left(\frac{\phi}{2}\right) d \phi=\frac{16}{h|\eta|} \frac{\omega_{s}}{\omega_{\text {rev }}}
$$

To obtain the phase-space area in $\mathrm{eV} \cdot \mathrm{s}$, one should multiply this quantity by the reference momentum $p_{0}$ and divide by the RF frequency $2 \pi f_{r f}$.

The region within the separatix is known as the RF bucket. Particles within this region of phase-space stay within this region of phase-space. The average momentum and phase of particles in the RF bucket can be changed be by adiabatically changing the fixed point 
of the bucket. The collection of particles that share the same RF bucket are referred to as a bunch. A particle beam is composed of many discrete bunches, one arriving after another, all with similar momentum.

The size of a particle bunch in phase-space is described by quantity known as the longitudinal emittance, which calculated using the product of the RMS momentum spread with the RMS temporal spread:

$$
\epsilon=\pi \sigma_{p} \sigma_{T}=\pi \frac{p_{0}}{2 \pi f_{r f}} \sigma_{\delta} \sigma_{\phi}
$$

A beam with a smaller longitudinal emittance is preferable because it facilitates the task of transporting the beam in the RF bucket. Nonlinear effects can distort or reduce the effective RF bucket area.

\subsection{Synchrotron Oscillation Motion}

In this section we examine the longitudinal dynamics of particles within the RF bucket. First we will show the shift in the synchrotron oscillation frequency with the synchrotron oscillation amplitude of a particle. Next we will show a perturbative solution to oscillatory particle trajectories.

The synchrotron oscillation period can be calculated as a function of maximum oscillation phase $\hat{\phi}$ by manipulating Eq. 1.11:

$$
\begin{gathered}
-\omega_{s}^{2} \sin (\phi)=\frac{d}{d t}\left[\frac{d \phi}{d t}\right]=\frac{d}{d \phi}\left[\frac{d \phi}{d t}\right] \frac{d \phi}{d t}=\frac{1}{2} \frac{d}{d \phi}\left[\left(\frac{d \phi}{d t}\right)^{2}\right] \\
\frac{d t}{d \phi}=\left[\int-2 \omega_{s}^{2} \sin (\phi) d \phi\right]^{-1 / 2}=\frac{1}{\sqrt{2} \omega_{s}} \frac{1}{\sqrt{\cos (\phi)-\cos (\hat{\phi})}} \\
T=\frac{4}{\sqrt{2} \omega_{s}} \int_{0}^{\hat{\phi}} \frac{d \phi}{\sqrt{\cos (\phi)-\cos (\hat{\phi})}}=T_{0} \frac{2}{\pi} K\left[\sin \left(\frac{\hat{\phi}}{2}\right)\right]
\end{gathered}
$$

where $T_{0}$ is the synchrotron period of small oscillations $T_{0}=2 \pi / \omega_{s}$ and $K$ is the complete

elliptic integral of the first kind $K[k]=\int_{0}^{\pi / 2} \frac{d u}{\sqrt{1-k^{2} \sin ^{2}(u)}}$. 
To further understand how the large oscillation trajectory differs from the small oscillation trajectory, we can expand the system perturbatively. For small $\phi$, Eq. 1.11 becomes:

$$
\ddot{\phi}=-\omega_{s}^{2} \sum_{k=0}^{\infty} \frac{(-1)^{k}}{(2 k+1) !} \phi^{2 k+1} \approx-\omega_{s}^{2}\left(\phi-\frac{1}{6} \phi^{3}+\ldots\right)
$$

Using the Poincare-Lindstedt method (see Ch. 2 of [3]), we can obtain a perturbative solution for Eq. 1.19 given by:

$$
\phi \approx \rho \sin \left(\omega_{s}(1+\sigma) t+\psi\right)+A_{3} \sin \left(3 \omega_{s}(1+\sigma) t+3 \psi\right)+\ldots
$$

For brevity let $s_{n} \equiv \sin \left(n \omega_{s}(1+\sigma) t+n \psi\right)$. We put Eq. 1.20 into Eq. 1.19 to obtain:

$$
\begin{aligned}
& \ddot{\phi} \approx-\omega_{s}^{2}\left[\left(\rho s_{1}+A_{3} s_{3}\right)-\frac{1}{6}\left(\rho^{3} s_{1}^{3}\right)\right] \\
& \ddot{\phi} \approx-\omega_{s}^{2}\left[\left(\rho s_{1}+A_{3} s_{3}\right)-\frac{1}{6}\left(\frac{3}{4} \rho^{3} s_{1}-\frac{1}{4} \rho^{3} s_{3}\right)\right]
\end{aligned}
$$

Applying the second-order derivation to Eq. 1.20 and setting the two sides equal, we obtain two equations:

$$
\begin{gathered}
-\omega_{s}^{2}(1+\sigma)^{2} \rho s_{1}=-\omega_{s}^{2}\left(\rho-\frac{1}{8} \rho^{3}\right) s_{1} \\
-9 \omega_{s}^{2}(1+\sigma)^{2} A_{3} s_{3}=-\omega_{s}^{2}\left(A_{3}-\frac{1}{24} \rho^{3}\right) s_{3}
\end{gathered}
$$

In Eq. $1.23 \sigma$ is negligible and we obtain:

$$
A_{3}=-\frac{1}{192} \rho^{3}
$$

We subtract $-\omega_{s} \rho s_{1}$ from each side of Eq. 1.22, hold $\sigma^{2}$ negligible, and solve for $\sigma$ :

$$
\sigma=-\frac{1}{16} \rho^{2}
$$

If we perform this expansion to higher orders of $\rho$ we can obtain terms with higher odd harmonics of $\omega_{s}$, obtain more precise calculations of the coefficients to these terms, and obtain a more precise calculation of the synchrotron tune shift $\sigma$. 


\subsection{Slipping Particles Motion}

In this section we examine the longitudinal dynamics of particles outside of the RF bucket by calculating the pertubative solution to the particle trajectories.

Particles with trajectories outside of the separatrix slip with respect to the RF bucket. The motion of these particles can be studied by writing Eq. 1.11 in a moving reference frame:

$$
\begin{aligned}
& \phi=\Omega t+\phi_{\circ}+\theta \\
& \ddot{\theta}=-\omega_{s}^{2} \sin \left(\Omega t+\phi_{\circ}+\theta\right) \\
& \ddot{\theta}=-\omega_{s}^{2}\left[\sin \left(\Omega t+\phi_{\circ}\right) \cos (\theta)+\cos \left(\Omega t+\phi_{\circ}\right) \sin (\theta)\right]
\end{aligned}
$$

For small $\theta$, we expand Eq. 1.26 perturbatively to study the oscillatory motion of the slipping particle:

$$
\begin{aligned}
& \ddot{\theta}=-\omega_{s}^{2}\left[\sum_{k=0}^{\infty} \frac{(-1)^{k}}{(2 k) !} \theta^{2 k} \sin \left(\Omega t+\phi_{\circ}\right)+\sum_{k=0}^{\infty} \frac{(-1)^{k}}{(2 k+1) !} \theta^{2 k+1} \cos \left(\Omega t+\phi_{\circ}\right)\right] \\
& \ddot{\theta} \approx-\omega_{s}^{2}\left[\sin \left(\Omega t+\phi_{\circ}\right)+\theta \cos \left(\Omega t+\phi_{\circ}\right)\right]
\end{aligned}
$$

The perturbative solution to Eq. 1.29 is of the form:

$$
\theta \approx B_{1} \sin \left(\Omega t+\phi_{\circ}\right)+B_{2} \sin \left(2 \Omega t+2 \phi_{\circ}\right)+\ldots
$$

For brevity let $S_{n} \equiv \sin \left(n \Omega t+n \phi_{\circ}\right)$ and $C_{n} \equiv \cos \left(n \Omega t+n \phi_{\circ}\right)$. We put Eq. 1.31 into Eq. 1.29 to obtain:

$$
\ddot{\theta} \approx-\omega_{s}^{-2}\left[S_{1}+B_{1} S_{1} C_{1}\right]
$$

We split Eq. 1.32 into two equations:

$$
\begin{aligned}
-\Omega^{2} B_{1} S_{1} & =-\omega_{s}^{-2} S_{1} \\
-4 \Omega^{2} B_{2} S_{2} & =-\frac{1}{2} \omega_{s}^{-2} B_{1} S_{2}
\end{aligned}
$$


From Eq. 1.33 we obtain:

$$
B_{1}=\left(\frac{\omega_{s}}{\Omega}\right)^{2}
$$

From Eq. 1.34 and Eq. 1.35 we obtain:

$$
B_{2}=\frac{1}{8}\left(\frac{\omega_{s}}{\Omega}\right)^{2} B_{1} \approx \frac{1}{8}\left(\frac{\omega_{s}}{\Omega}\right)^{4}
$$

The perturbation can be expanded to higher orders and the coefficients of $B_{n}$ are of the

order $\left(\frac{\omega_{s}}{\Omega}\right)^{2 n}$. The perturbation for small $\theta$ is better expressed as a perturbation of small $\left(\frac{\omega_{s}}{\Omega}\right)^{2}$. If the slipping particle trajectory is close to the separatrix then $\Omega$ is small and the nonlinear oscillatory motion is large. For a particle far from the reference momentum, the force from the RF cavity does not add up coherently and the motion approaches that of a coasting beam.

The motion of stable slip-stacking particles, which will be described in Chapter 3, is a combination of synchrotron motion and slipping motion.

\subsection{Acceleration \& Focusing Motion}

Particle beams are accelerated by gradually changing the resonant frequency of the RF cavities. The momentum of the stable particles change to match the new reference momentum. For a linearly increasing RF frequency, we transform the coordinates into the accelerating reference frame:

$$
\dot{\delta}=f_{\text {rev }} V_{\delta}\left[\sin (\phi)-\sin \left(\phi_{s}\right)\right], \dot{\phi}=2 \pi f_{r e v} h \eta \delta
$$

The stable fixed point for these equations of motions is $\phi=\phi_{s}, \delta=0$, which corresponds to a particle whose momentum exactly follows the reference momentum and whose phase changes each revolution to exactly match the change in the RF frequency. The separatrix changes depending on $\phi_{s}$ and this region of phase-space is referred to as the running $\mathrm{RF}$ 
bucket. The greater the magnitude of $\phi_{s}$, the greater the acceleration and the smaller the RF bucket.

Fig. 1.5 shows the motion of particles inside and outside the running RF bucket. Particles within the separatrix remain synchronized with the RF and accelerate linearly. Particles outside the separatrix, however, are not accelerated and deviate increasingly from the accelerating reference momentum. The transversely bending and focusing magnetic elements of the particle accelerator ring increase in field strength to follow the accelerating beam and consequently the particles that are not accelerated are lost. The total range of momentum that a particle accelerator ring can support due to transverse dynamics is known as the momentum aperture of that ring. The total range of momentum that an RF bucket can store simultaneously is known as the momentum acceptance.

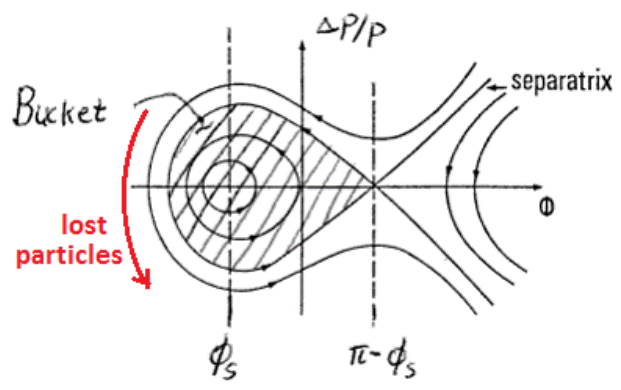

Figure 1.5: Phase space trajectories associated with the running bucket. Only particles inside the separatrix follow the accelerating reference frame.

In [2], the running bucket area factor $\alpha_{b}\left(\phi_{s}\right)$ gives the ratio between the stable phasespace area of the running bucket and the stationary bucket:

$$
\alpha_{b}\left(\phi_{s}\right)=\frac{\mathcal{A}_{b}}{\mathcal{A}_{0}}=\frac{1}{4 \sqrt{2}} \int_{\phi_{u}}^{\pi-\phi_{s}}\left|\cos (\phi)+\cos \left(\phi_{s}\right)-\left(\pi-\phi-\phi_{s}\right) \sin \left(\phi_{s}\right)\right|^{1 / 2} d \phi
$$

where $\phi_{u}$ is found from the transcendental equation

$$
\cos \left(\phi_{u}\right)+\phi_{u} \sin \left(\phi_{s}\right)=-\cos \left(\phi_{s}\right)+\left(\pi-\phi_{s}\right) \sin \left(\phi_{s}\right)
$$


The running bucket area factor is plotted in Fig. 1.6 and is approximated with

$$
\alpha_{b}\left(\phi_{s}\right) \approx \frac{1-\sin \left(\phi_{s}\right)}{1+\sin \left(\phi_{s}\right)}
$$

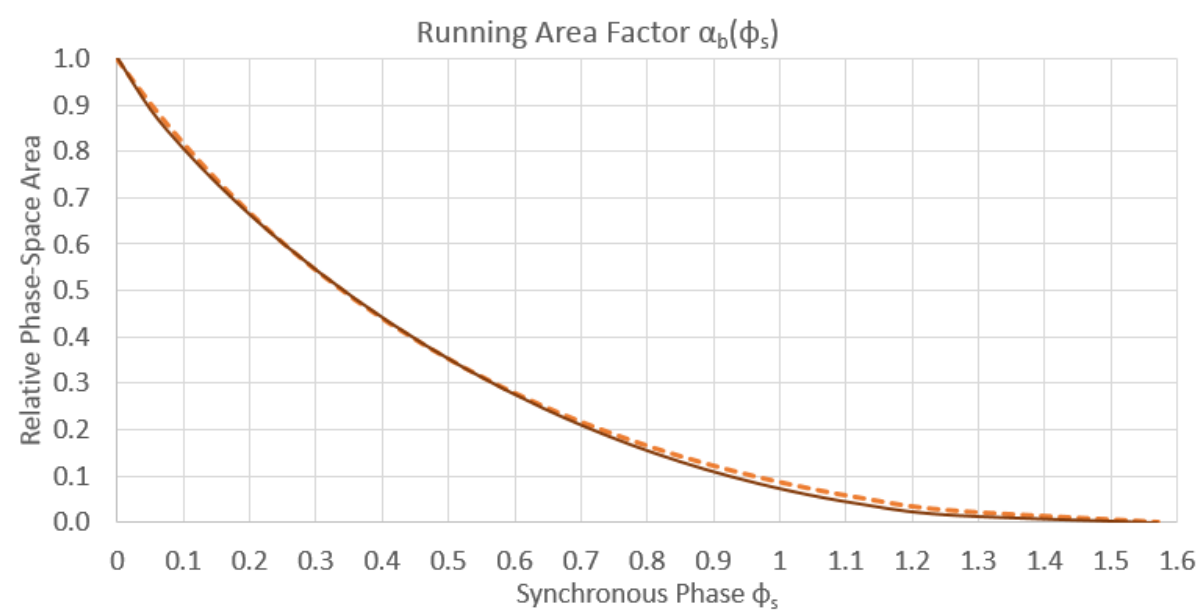

Figure 1.6: The ratio between the running bucket area and the corresponding stationary bucket area, as a function of the synchronous phase $\phi_{s}$ of the running bucket. The exact value is shown in a dark orange line and the approximate value is shown in a dashed orange.

The parameters $f_{r e v}, V_{\delta}$, and $\eta$ in Eq. 1.37 generally change with acceleration but and can be treated adiabatically so long as they change slowly compared to the synchrotron frequency. This approximation breaks down when $\eta$ is small because the focusing is weaker and this is referred to as quasi-isosynchronous condition. In the quasi-isosynchronous case, the dependency of the revolution frequency on $\eta$ is expanded to include the second order term:

$$
\frac{T-T_{r e v}}{T_{\text {rev }}} \approx 0+\frac{1}{T_{\text {rev }}} \frac{\partial T}{\partial \delta} \delta+\frac{1}{T_{\text {rev }}} \frac{\partial^{2} T}{\partial \delta^{2}} \frac{\delta^{2}}{2}=\eta_{0} \delta+\eta_{1} \delta^{2}
$$

When a particle accelerator crosses transition energy, where $\eta=0$, it can be a significant source of longitudinal emittance growth. At transition energy, acceleration is still occurs but there is no linear longitudinal focusing force. After crossing transition energy, the longitudinal focusing force will have changed orientation and the stable fixed point will have 
changed by $\pi$. The process complicates particle motion and decoherence in the ensemble of particles increases the longitudinal emittance.

\subsection{Summary}

In this chapter we show that longitudinal dynamics of single fixed-frequency RF cavity is identical to that of simple pendulum. The separatrix of the simple pendulum separates the stable phase-space area from the unstable phase-space area. The stable particles are in the RF bucket and the unstable particles are slipping with respect to the RF bucket. The ensemble of particles that share the same RF bucket are referred to as a bunch and the size

of a bunch is measured by its longitudinal emittance. In order to change the energy of a particle bunch the RF frequency must be changed gradually and only the particles inside the separatrix will be accelerated successfully. 


\section{CHAPTER 2}

\section{Fermilab Accelerator Complex}

In this chapter, I provide an overview of the Fermilab proton accelerator complex. Although the rest of the dissertation focuses on the dynamics of slip-stacking, it is important to understand the role that slip-stacking plays in delivering high-intensity proton beams for the lab. Slip-stacking occurs in the Fermilab Recycler, receives beam from the Fermilab Booster, and delivers beam to the Fermilab Main Injector. In order to increase $120 \mathrm{GeV}$ proton beam power at Fermilab, it is essential to reduce losses from slip-stacking in the Recycler. This chapter also provides a brief introduction to some of the accelerator concepts not covered in the previous chapter. Lastly, this chapter provides an up-to-date overview of the status of the proton accelerator complex that helps clarify the challenges for future upgrades in proton beam power.

\subsection{Overview}

The overarching research priority for the proton accelerator complex at Fermilab is to increase the proton beam power, the number of protons accelerated (to a given energy) per unit time. Increases in beam power come with increasingly strict limits on the highenergy proton loss rate in order to keep nuclear activation of the particle accelerator below acceptable limits [4-6]. Proton beam power at Fermilab is advanced in three sequential stages of accelerator upgrades, referred to as the Proton Improvement Plan (PIP), PIP-II, 
and PIP-III. PIP is mostly complete, PIP-II is designed in detail [7], and PIP-III is still being envisioned. Over the course of this chapter, the PIP, PIP-II, and PIP-III upgrades will be described.

Fig. 2.1 shows the proton accelerator complex at Fermilab. The beam begins from an $H^{-}$ion source and is rapidly accelerated to a kinetic energy of $400 \mathrm{MeV}$ using the Linac (linear accelerator). The $400 \mathrm{MeV} H^{-}$beam is injected into the Fermilab Booster by using a carbon foil to strip away the two electrons and this $400 \mathrm{MeV}$ proton beam is accumulated in the Booster. The Booster accelerates this beam up to an energy of $8 \mathrm{GeV}$, where it can be used immediately for a fixed-target experiment or transferred to a larger accelerator ring. The 8-GeV fixed-target experiments include the low-energy neutrino program [8], the muon program $[9,10]$, and the test-beam facilities MCenter and MTest.

The Booster has $84 \mathrm{RF}$ buckets and the sequence of bunches occupying these buckets are collectively referred to as a Booster batch. The Recycler and Main Injector each have $588 \mathrm{RF}$ buckets and have a circumference seven times that of the Booster. At $8 \mathrm{GeV}$, the three rings have the same RF frequency of approximately $53 \mathrm{MHz}$ and the Booster beam is transferred to the Recycler bucket-to-bucket.

The Recycler is used to accumulate multiple Booster batches and that beam is extracted to the Main Injector when the Recycler cannot accumulate any more. The Main Injector accelerates that $8-\mathrm{GeV}$ beam to $120 \mathrm{GeV}$ and delivers it to a high-energy fixed target experiment. The $120-\mathrm{GeV}$ beam either goes to carbon target for the Neutrinos at Main Injector (NuMI) beamline [11-13] or a hydrogen-deuterium target for the SeaQuest experiment [14]. The NOvA experiment, a long-baseline neutrino oscillation measurement using the NuMI beamline, is currently the primary user of the Fermilab proton complex.

Fig. 2.2 shows how batches are accumulated in the Recycler before acceleration in the Main Injector. Six batches fill up the available azimuthal space of the Recycler, but slip- 


\section{Fermilab Accelerator Complex}

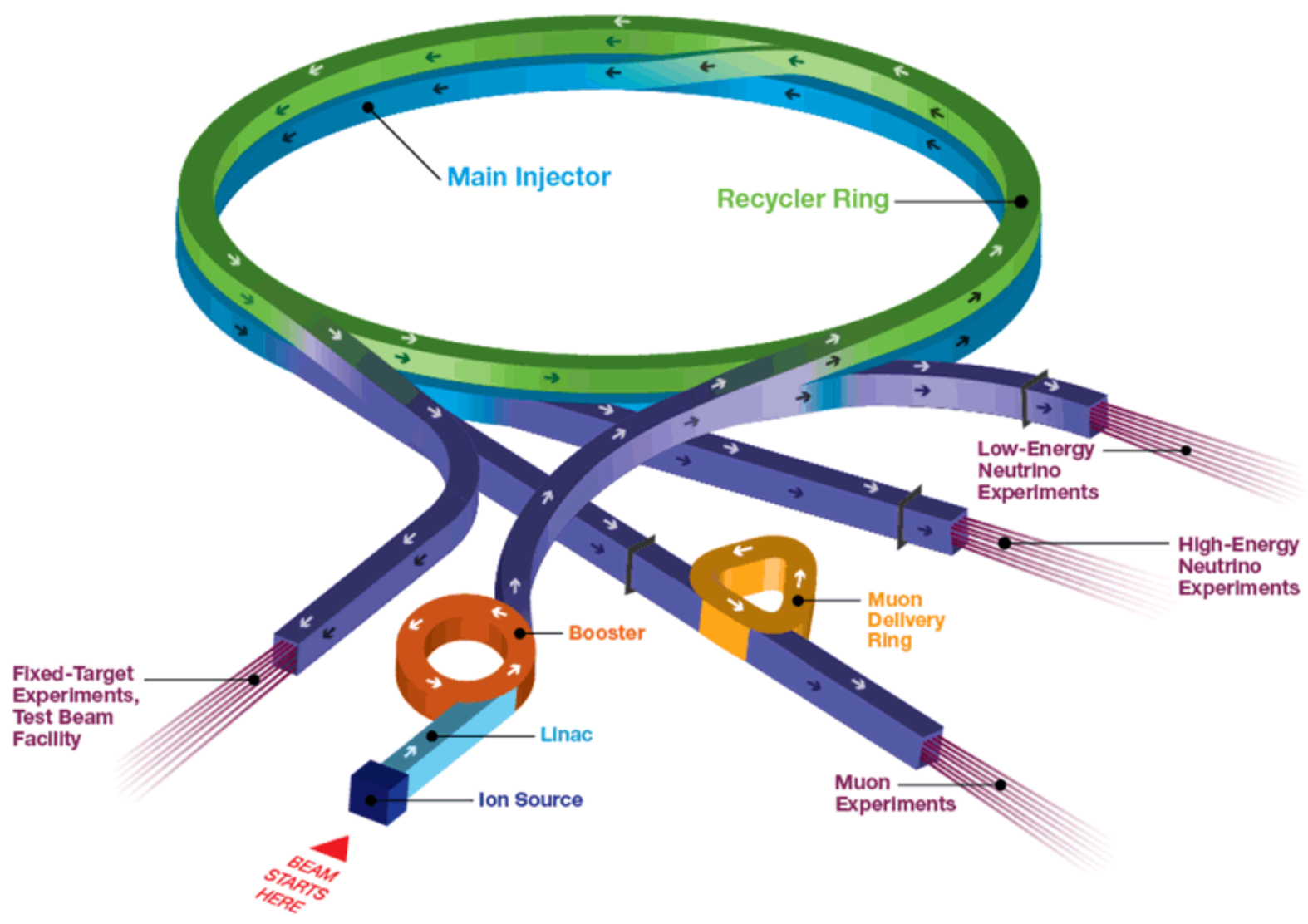

Figure 2.1: Conceptual diagram of the particle accelerators, storage rings, transfer lines, and experiments of the Fermilab proton accelerator complex. 
stacking enables twelve batches to be stored in the Recycler by employing two beams that have different momenta but overlap azimuthally. A detailed description of this accumulation scheme is found in the next chapter. The Booster cycle-rate is $15 \mathrm{~Hz}$ which means that every $66 \mathrm{~ms}$ it produces a new $8-\mathrm{GeV}$ Booster batch and it takes 0.8 seconds to accumulate 12 batches in the Recycler. The Main Injector cycle currently takes 1.33 seconds, taking half that time to accelerate the $8-\mathrm{GeV}$ beam to $120 \mathrm{GeV}$ and half to ramp-down its magnets to the field strength compatible with the next $8-\mathrm{GeV}$ beam. The Main Injector cycle time does not require time for beam accumulation because the beam accumulation occurs in the Recycler while the Main Injector ramps. Slip-stacking in the Recycler is currently under commissioning [15] but has already broken the world record in neutrino beam power [16]. Fig. 2.3 shows the current progress on the $700 \mathrm{~kW}$ beam power upgrade.

\subsection{Injector \& Linac}

The $H^{-}$portion of the accelerator complex consists of an injector and a Linac. The injector supplies an initial source of $H^{-}$ions using a magnetron-based source and accelerates that beam to an energy of $725-760 \mathrm{keV}$. In 1968, a Cockcroft-Walton injector was installed which used a series of voltage stages to place the magnetron source $\sim-750 \mathrm{kV}$ relative to ground and use DC for the initial acceleration [18]. In 2012, however as part of the PIP upgrade the Cockcroft-Walton injector was replaced with a new injector based on design from Brookhaven National Laboratory (BNL). The new injector uses a Radiofrequency Quadrupole (RFQ) to accelerate and focus the $H^{-}$beam to $750 \mathrm{keV}$ with $200 \mathrm{MHz} \mathrm{RF}$ voltage [19-21]. Attached to the RFQ injector are two identical magnetron-based sources. The second one may be used when the performance of the first one falters [22]. Fig. 2.4 shows a diagram of the RFQ injector. The Low-Energy Beam Transport (LEBT) has two transversely focusing solenoids that are used to transport the beam from the the magnetron 


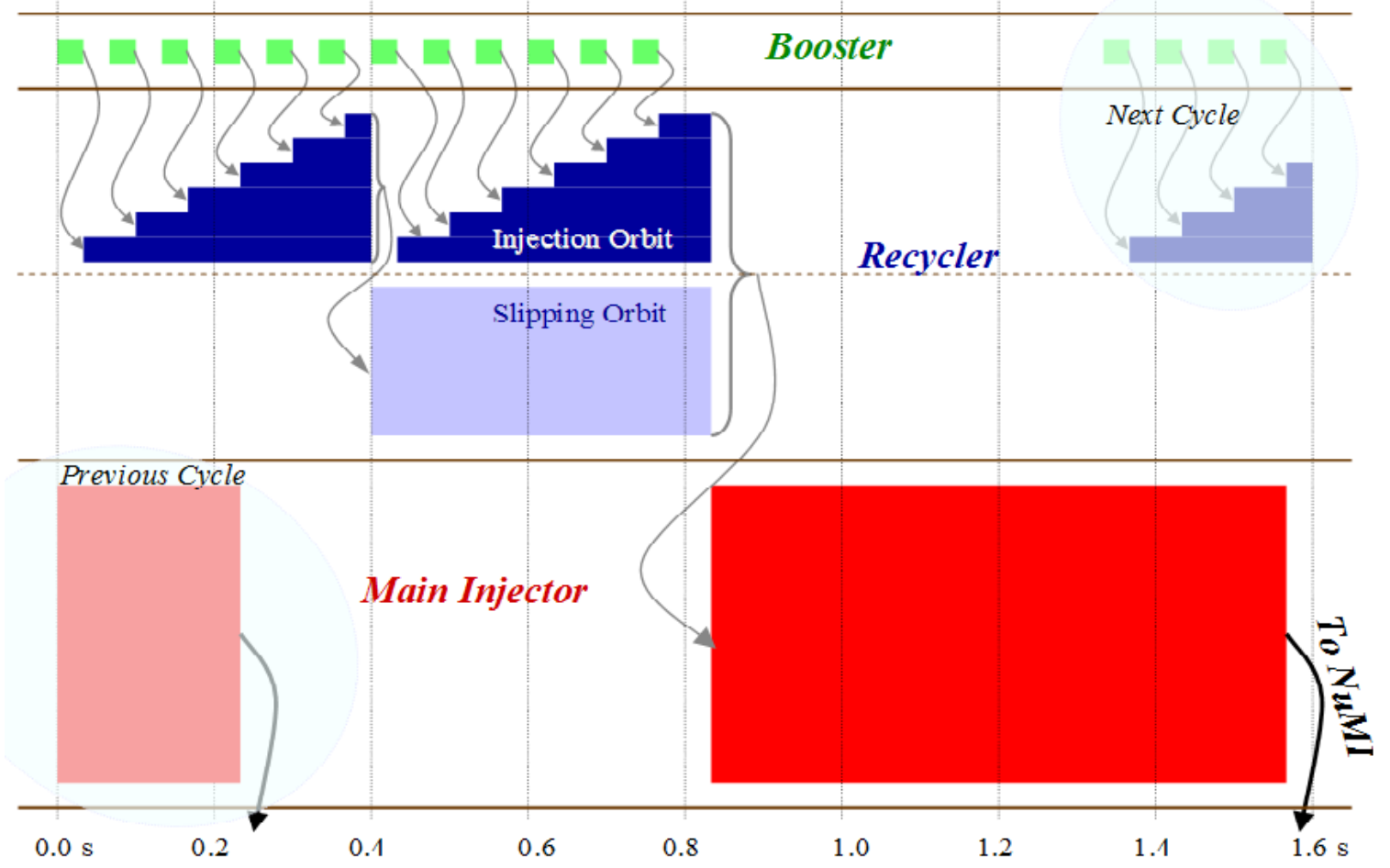

Figure 2.2: The diagram shows the beam intensity in each ring on the vertical axis and time on the horizontal axis. The Recycler accumulates Booster batches while the Main Injector completes it ramp cycle. [17] 


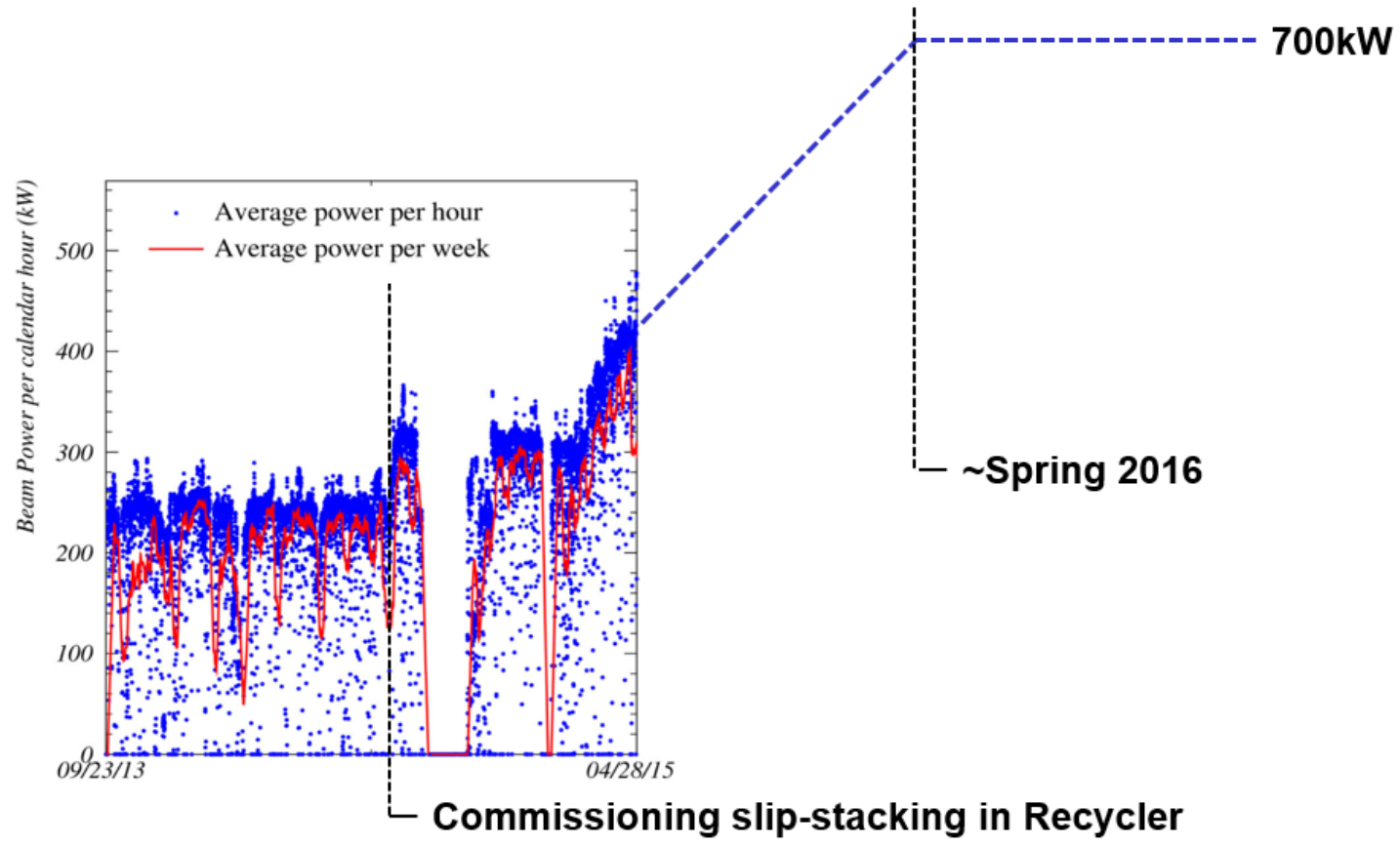

Figure 2.3: Beam power has improved during the initial stages of the commissioning the Recycler, which is expected to be completed this spring. [15]

into the RFQ [23]. Designed to match the beam from the RFQ to the Linac, the MediumEnergy Beam Transport (MEBT) is composed of a doublet of quadrupoles for focusing the beam and an RF cavity for bunching the beam [23].

The Linac accelerates the $750 \mathrm{keV} H^{-}$beam to $116.5 \mathrm{MeV}$ using a $75 \mathrm{~m}$ sequence of five drift-tube linac (DTL) modules and accelerates from 116.5 MeV to $400 \mathrm{MeV}$ using a $64 \mathrm{~m}$ sequence of seven side-coupled linac (SCL) modules. Each linac module is composed of many accelerating cells which are coupled together and a driven by the same input signal. The coupling between the accelerating cells ensures that each cell has a $\pi / 2$ phase-shift and the arrival time of the beam coincide with the accelerating portion of the RF mode. The $H^{-}$beam moves semi-relativistically, so the cell length must also be designed so that the transit time matches that $\pi / 2$ phase-shift between cells. The DTL modules use a 201.24 MHz signal driven by a 5MW power amplifer and the SCL modules use a $804.96 \mathrm{MHz}$ signal 


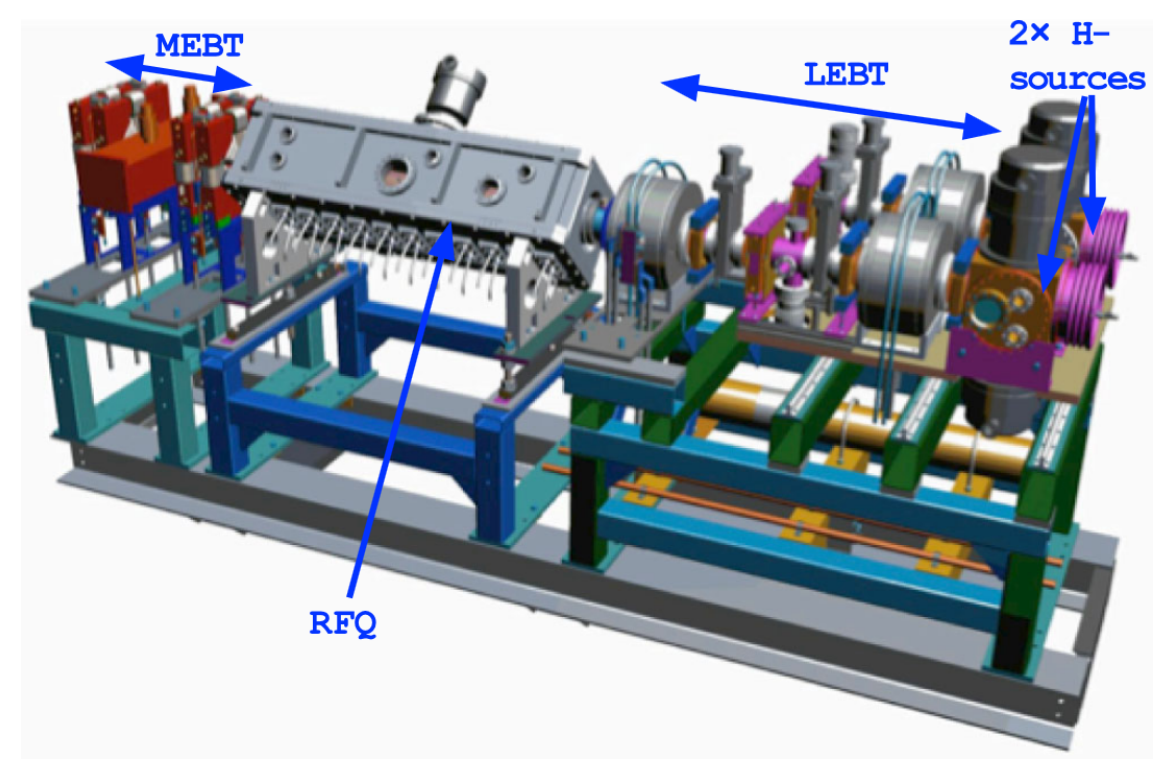

Figure 2.4: Model of the new RFQ-Injector. The beam begins in one of two $H^{-}$magnetrons, travels to the RFQ through the LEBT solenoids, accelerates to $750 \mathrm{keV}$ in the RFQ, and matches to the Linac with the MEBT. [19]

driven by a $12 \mathrm{MW}$ Kylstron amplifier. There are significant concerns about the long-term reliability of the DTL modules [24] and the entire Linac is slated to be replaced as part of the PIP-II upgrade [7].

\subsection{Booster}

Some key parameters of the Fermilab Booster RF system and magnetic lattice are given in Table 2.1. The Booster lattice is composed of 24 nearly identical magnetic cells that each include four bending magnets and a drift region (used for RF cavities or other accelerator elements). Fig. 2.5 shows the "FDOODFO" sequence of magnets contained in each of the 24 cells and Fig. 2.6 shows the two types of Booster bending magnets. Magnets of this type are referred to as gradient dipole magnets, because there is a strong dipole field changing in magnitude with horizontal displacement. The strong dipole field bends the beam around the arc and the quadrupole component of this field (adjacent magnets alternating in 


\begin{tabular}{|c|c|c|c|}
\hline Circumference & $473.8 \mathrm{~m}$ & Number of Magnetic Cells & 24 \\
\hline Number of Protons & $4.3 \times 10^{12}$ & Bending Magnets per Cell & 4 \\
\hline Energy & $0.4-8 \mathrm{GeV}$ & Magnetic Elements in Cell & FDOODFO \\
\hline Frequency & $37.77-52.81 \mathrm{MHz}$ & Bending Magnet Length & $2.9 \mathrm{~m}$ \\
\hline Cycle-rate & $15 \mathrm{~Hz}$ & Standard Cell Length & $19.74 \mathrm{~m}$ \\
\hline Harmonic Number & 84 & Maximum Horizontal $\beta$ & $33.7 \mathrm{~m}$ \\
\hline Transition gamma & 5.45 & Maximum Vertical $\beta$ & $20.5 \mathrm{~m}$ \\
\hline Maximum Voltage & $1 \mathrm{MV}$ & Maximum Dispersion & $3.2 \mathrm{~m}$ \\
\hline Number of Cavities & 19 & Betatron Tunes & 6.7 \\
\hline
\end{tabular}

Table 2.1: RF and magnet parameters of the Booster ring. [25, 26]

sign) focuses the beam transversely. The number of transverse oscillations per revolution, known as the betatron tune, is picked carefully to minimize parametric resonances that are driven by nonlinearities and field errors. The ring is generally dispersive, causing the higher momentum particles to travel along outer orbits relative to the lower momentum particles.

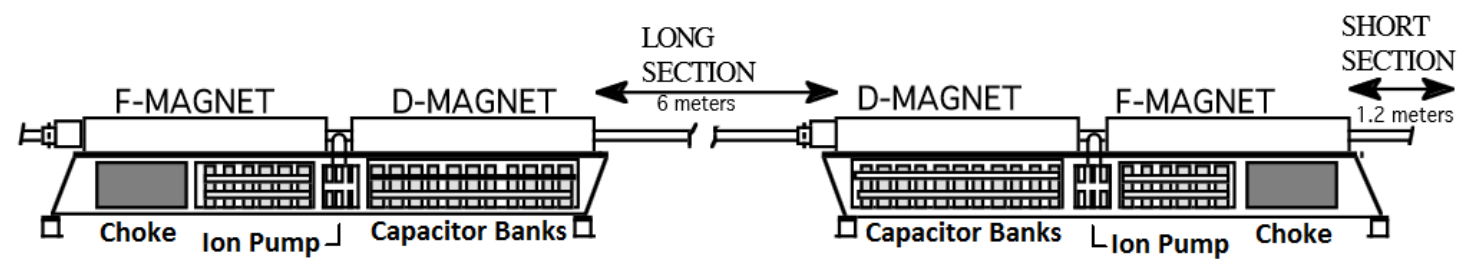

Figure 2.5: One of Twenty-Four magnet cells that bend and transversely focus the beam. [25]

Fig. 2.7 shows how the carbon foil is used to strip away the electrons from the $H^{-}$ion beam and leave a proton beam injected into the Booster [25]. The limiting factor on proton bunch intensity in the Booster is the loss-rate at that bunch-intensity. In particular, the space-charge forces of the high-intensity proton bunch change the transverse focusing of the beam. Consequently, there is a betatron tune spread that makes it more difficult to avoid transverse parametric resonances. Fig. 2.8 from [27] shows the increased particle loss and transverse size as more beam is injected. 

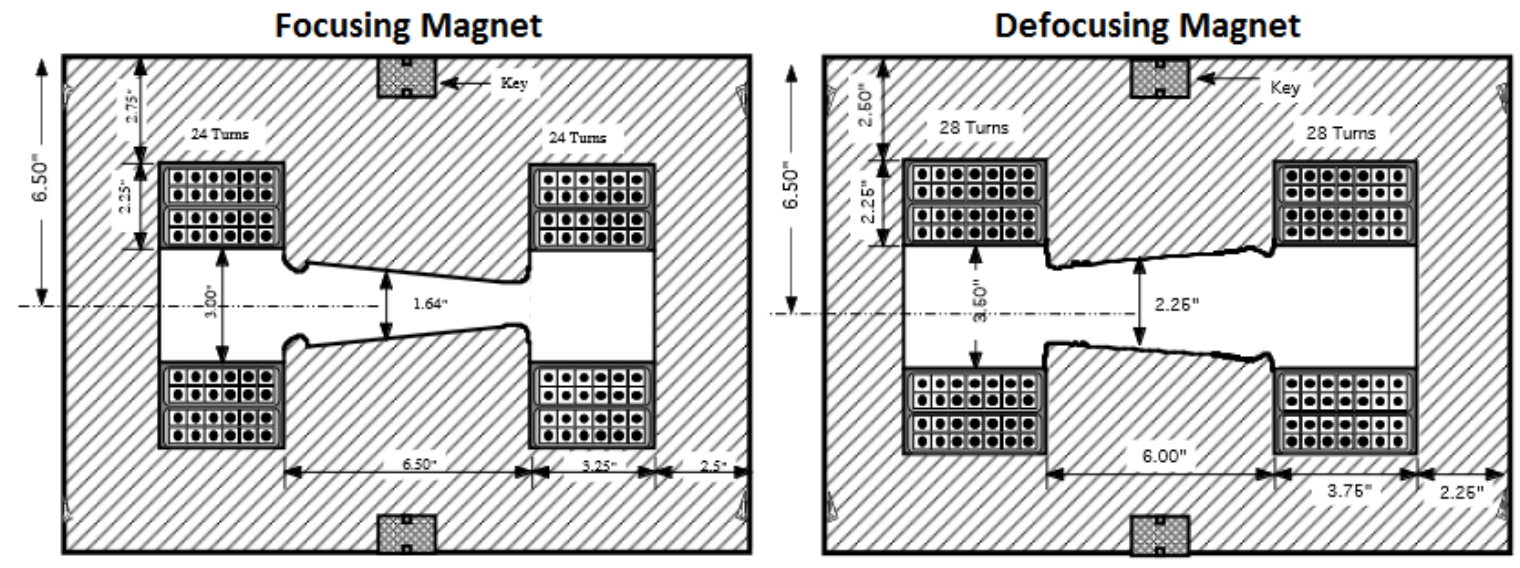

Figure 2.6: The gradient dipole magnets that horizontally focus and vertically defocus the beam (left) and the gradient dipole magnets that horizontally defocus and vertically focus the beam (right). The effect of both magnets in sequence results in a net focusing in the transverse plane. [25]

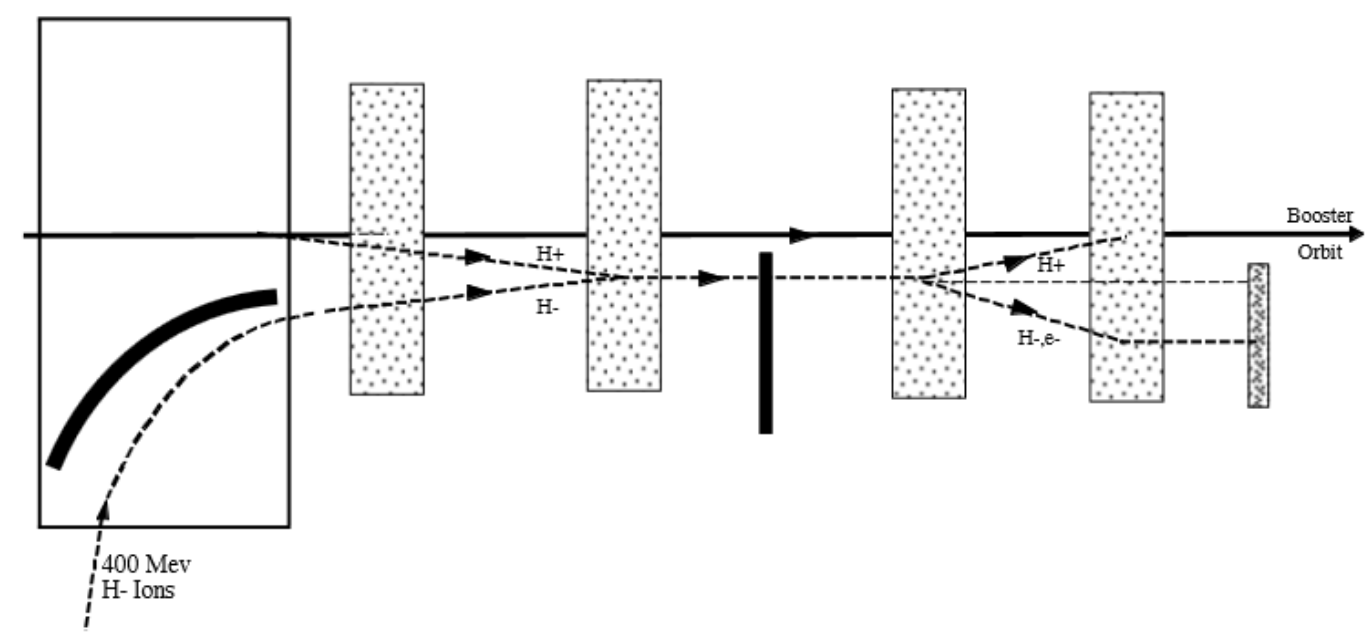

Figure 2.7: The $H^{-}$ion beam is injected so it coincides with the stored proton beam at the carbon stripping foil. The remaining $H^{-}$ion beam is directed into a beam dump. [25] 

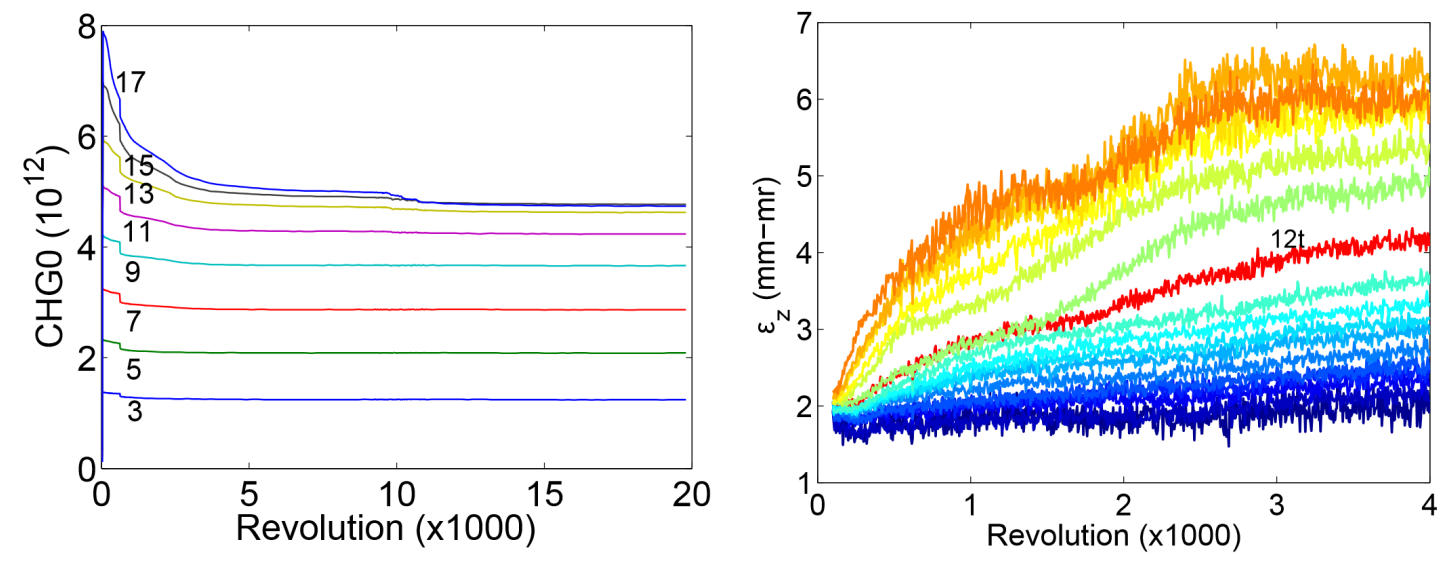

Figure 2.8: (left) Total number of protons stored in the Booster over time. Each line represents a different number of revolutions used to initially fill the Booster. (right) Normalized vertical emittance (size in vertical phase-space) as a function of time and the number of revolutions used to fill the Booster. [27]

The Booster gradient dipole magnets modulate in magnitude every $15 \mathrm{~Hz}$, following a sinusoidal waveform (with a DC offset). The entire magnetic lattice is set up as a resonant circuit with current flowing between the magnets and a capacitor banks every $15 \mathrm{~Hz}[25]$. The dipole field is not constant during either injection or extraction from the Booster. Currently the beam from the $805 \mathrm{MHz}$ Linac is injected when the dipole field is minimal and there is no RF focusing applied in the Booster. After the injected beam debunches, the total RF voltage is increased adiabatically. The increase in total RF voltage is accomplished using a technique called paraphasing [25] in which two sets of RF cavities are powered with opposite phases and the total RF voltage increases as the phases are brought together. An improved injection scheme is under development where the beam will be injected before the dipole field minimum and no debunching will be used before RF capture [6,26].

The Booster has $1955 \mathrm{kV}$ cavities that provide a total accelerating voltage of $1 \mathrm{MV}$. The Booster RF cavities have ferrite tuners that allow the RF frequency to sweep from 37.77 $\mathrm{MHz}$ to $52.81 \mathrm{MHz}$ and accelerate the beam each cycle. However the RF cavities are 40 years 
old and the pace of operating at $15 \mathrm{~Hz}$ generates heating that undermines reliability [28]. Therefore, as part of the PIP project [29] these RF cavities are being refurbished and their water-cooling systems are being upgraded. Simultaneously replacement RF cavities are being tested $[29,30]$ because the current RF cavities are not expected to last beyond 2025 [24]. As part of the PIP-II upgrade, there is the proposal to increase the Booster cycle-rate to $20 \mathrm{~Hz}$ which will require greater $\mathrm{RF}$ voltage and $\mathrm{RF}$ power dissipation. The $20 \mathrm{~Hz}$ Booster has implications for slip-stacking, which are discussed in the chapters that follow, and also increases the beam power available for $8-\mathrm{GeV}$ experiments.

A second harmonic cavity is being developed for the Booster [31] that will increase the injection capture efficiency and also aid in transition crossing in the Booster [32]. Crossing the transition energy at $5.1 \mathrm{GeV}$ is a major source of beam loss in the Booster and also a source of longitudinal emittance growth that can lead to losses in the Recycler and Main Injector. Another proposed transition crossing scheme requires a temporary increase in Booster RF voltage known as a voltage jump [33,34].

The rapid acceleration of the Booster beam causes it to have a large momentum spread relative to its temporal spread. To inject the Booster beam efficiently into the Recycler for slip-stacking, it is desirable to rotate the beam in longitudinal phase-space so that it has a smaller momentum spread. The Booster bunch rotation is performed via quadrupole excitation of the synchrotron oscillation [35]. The quadrupole excited is based on a voltage modulation technique developed at BNL [36] and previously studied at the Indiana University Cyclotron Facility [37]. The RF voltage is modulated at twice the synchrotron frequency and this drives a longitudinal quadrupole resonance. The longitudinal equation of motion is given by:

$$
\ddot{\phi}=-\omega_{s}^{2}\left[1+\Lambda \sin \left(2 \omega_{s} t\right)\right] \sin (\phi)
$$

Every quarter synchrotron period the bunch alternates between a state with a minimized 
temporal spread and a minimized momentum spread. Every half synchrotron period the distortion becomes more extreme and significant nonlinearities are observed. Fig. 2.9 shows a simulated progression of the Booster bunch rotation process for $25 \%$ amplitude modulation. The Booster bunch rotation is actively tuned to minimize losses and the tuning parameters depend on bunch intensity and beam quality.
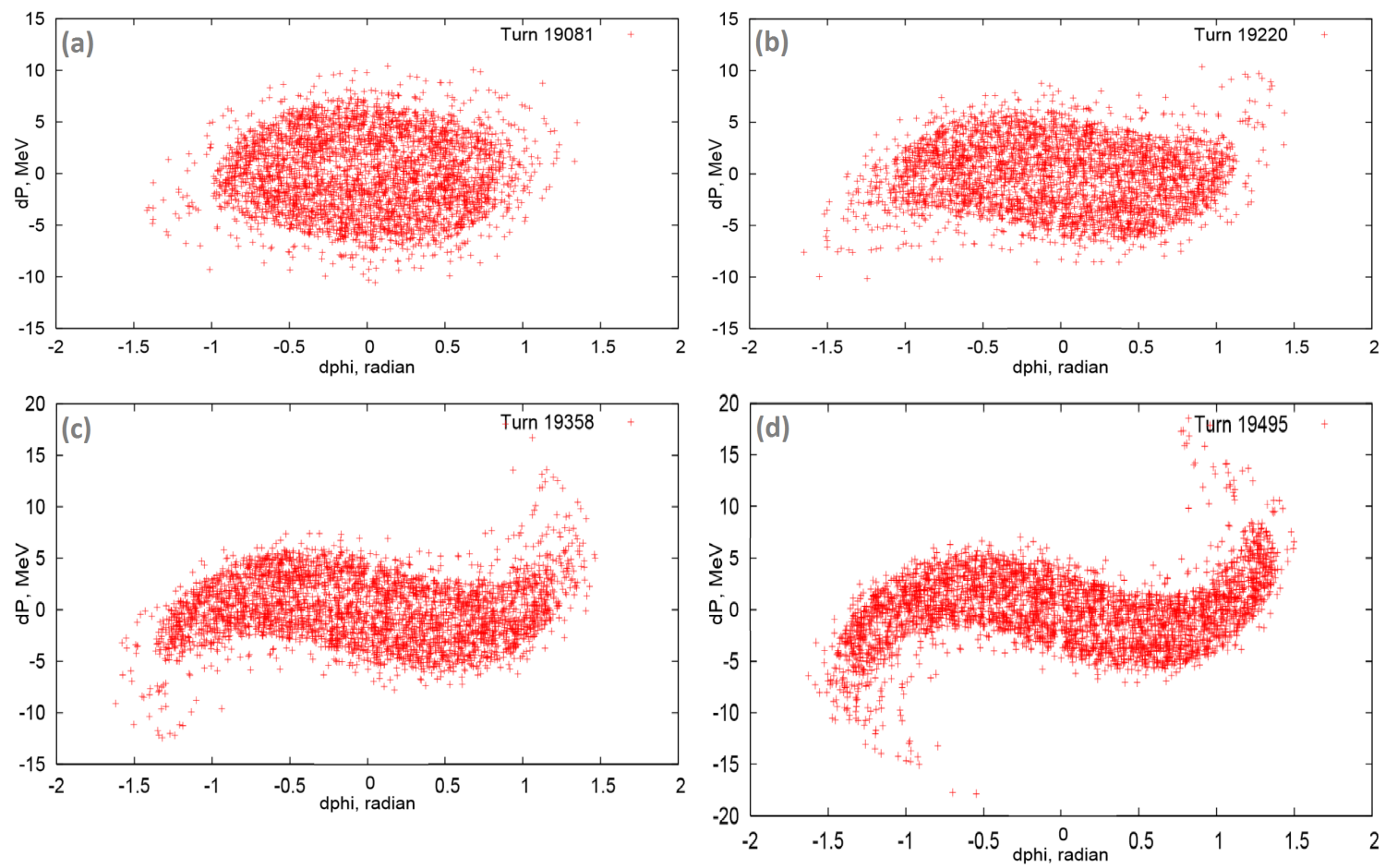

Figure 2.9: Longitudinal phase-space of a simulated Booster beam under the influence of a $25 \%$ modulation in voltage at twice the synchrotron frequency. The plots are shown as half-synchrotron period intervals and proceed from (a) to (d). [35]

\subsection{Recycler \& Main Injector}

Some key parameters of the Fermilab Recycler and Fermilab Main Injector are given in Table 2.2. Fig. 2.10 shows the layout of the magnetic lattice for the Main Injector as well as for the Recycler which was designed to follow the same layout $[38,40]$. The magnetic cells 


\begin{tabular}{|l|l|l|}
\hline Parameter & Recycler & Main Injector \\
\hline Circumference & $3319.400 \mathrm{~m}$ & $3319.415 \mathrm{~m}$ \\
Number of Protons & $51.6 \times 10^{12}$ & $51.6 \times 10^{12}$ \\
Energy & $8 \mathrm{GeV}$ & $8-120 \mathrm{GeV}$ \\
Frequency & $52.811 \mathrm{MHz}$ & $52.811-53.104 \mathrm{MHz}$ \\
Harmonic Number & 588 & 588 \\
Transition gamma & 20.7 & 21.8 \\
Maximum Total Voltage & $0.2 \mathrm{MV}$ & $4 \mathrm{MV}$ \\
Number of Cavities & 2 & 18 \\
Cycle Yime & $0.8 \mathrm{~s}($ fill $)$ & $1.333 \mathrm{~s}(\mathrm{ramp})$ \\
Maximum $\beta$ & $55 \mathrm{~m}$ & $57 \mathrm{~m}$ \\
Maximum Dispersion & $2 \mathrm{~m}$ & $1.9 \mathrm{~m}$ \\
Horizontal Betatron Tune & 25.425 & 26.425 \\
Vertical Betatron Tune & 24.415 & 25.415 \\
\hline
\end{tabular}

Table 2.2: RF and magnet parameters of the Recycler and the Main Injector. [5, 38]

come in three varieties - arc cells with dipoles and quadrupoles, straight-section cells with quadrupoles and accelerator components, and disperson-suppressor cells which resemble the arcs cells but are designed to lower dispersion in the straight sections. The Recycler arcs use gradient dipole magents for bending and focusing, similar to the Boosters gradient dipole magnets shown in Fig. 2.6. Unlike the Booster, the Recycler uses primarily permanent magnets composed of magnetized strontium ferrite bricks (with nickel-steel "compensator" strips and low-carbon steel pole-tips) [41]. The Main Injector arcs are composed of separate dipole and quadrupole magnets, shown in Fig. 2.11. In the Main Injector arc cells, there are two dipole magnets between adjacent quadrupoles, which alternate between horizontally focusing quadrupoles and horizontally defocusing quadrupoles.

Both the Main Injector and the Recycler also have powered correction magnets - dipoles, quadrupoles, and sextupoles - for making fine adjustments to the accelerator lattice. The 


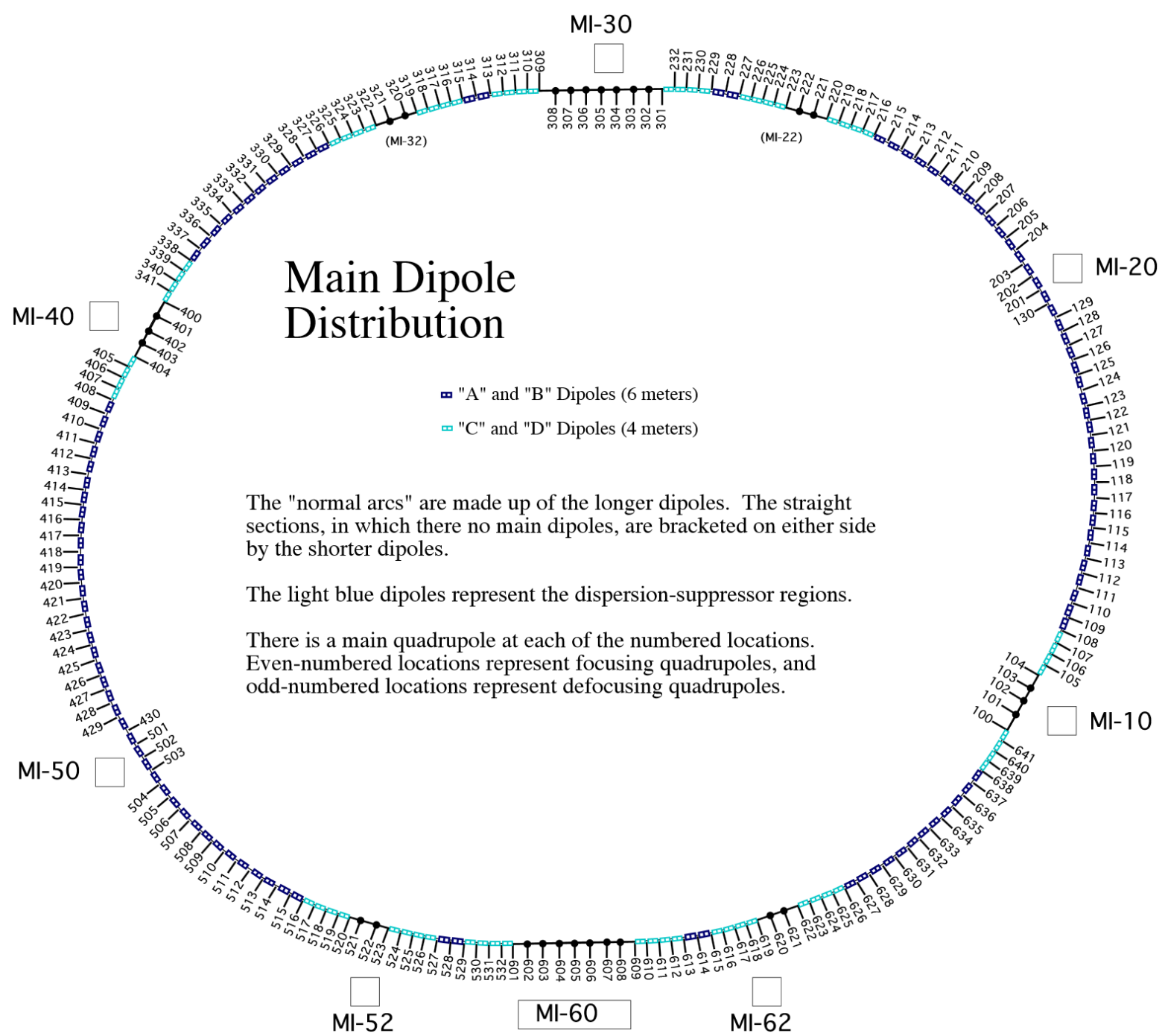

Figure 2.10: Distribution of arc cells (dark blue), dispersion-suppressor cells (light blue), and straight sections in the Main Injector. The Recycler follows a very similar layout. [39] 

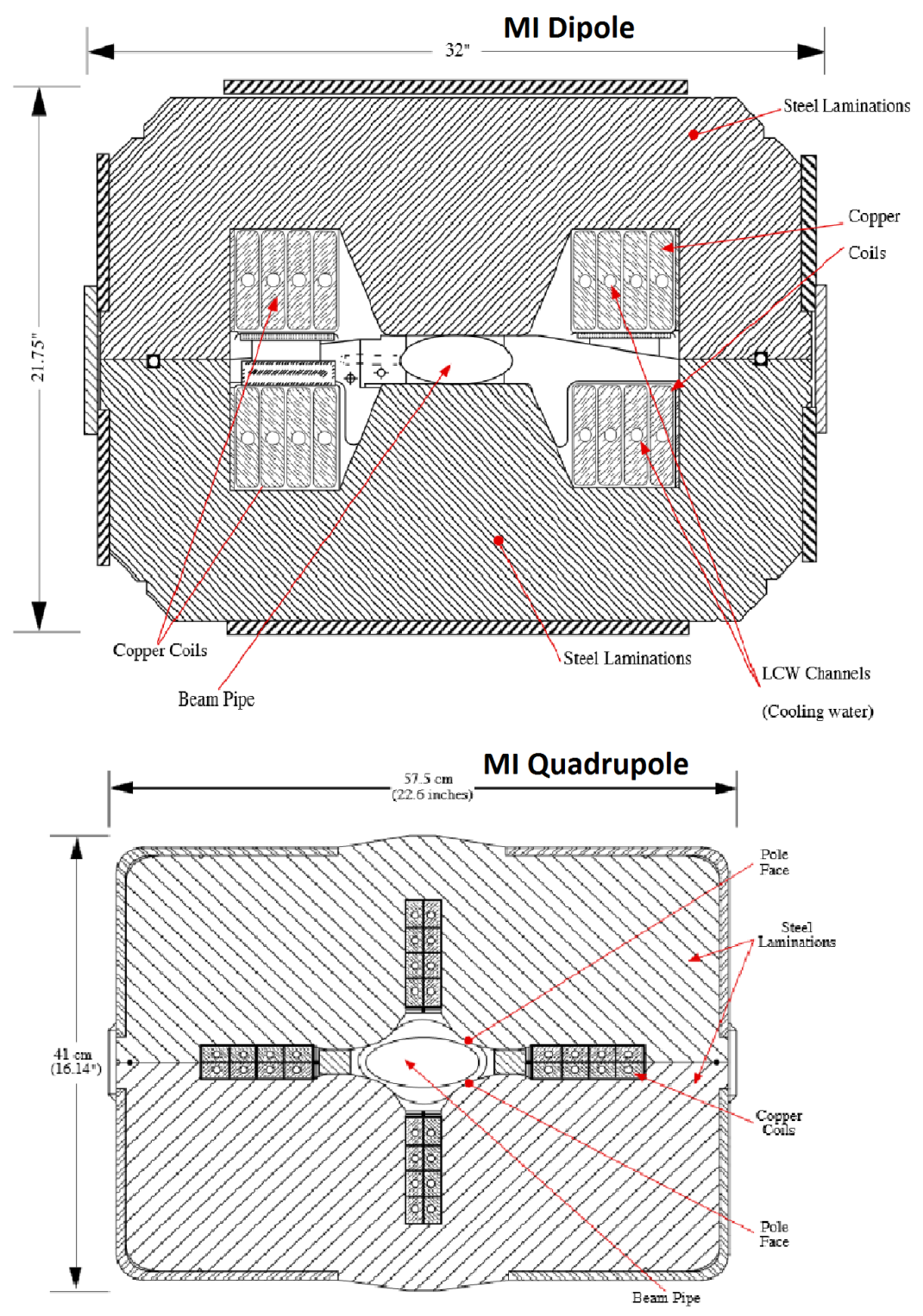

Figure 2.11: Main Injector dipole magnet (top) and Main Injector quadrupole magnet (bottom). The two magnets are shown on the same scale. [39] 

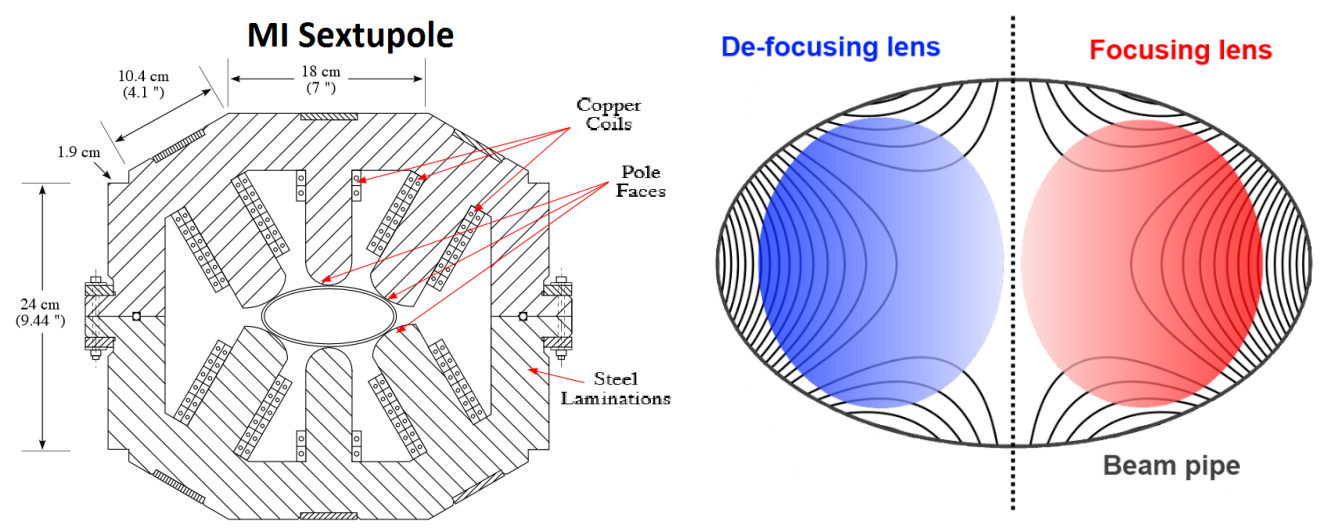

Figure 2.12: (left) Main Injector sextupole magnet on the same scale as Fig. 2.11. (right) The effective transverse focusing of the magnet depends on the horizontal beam position, which depends on beam momentum wherever there is dispersion. [1,39]

correction dipoles can be used to make a local change in orbit and the correction quadrupoles can be used to modify the betatron tune. Sextupoles magnets, like the one shown in Fig. 2.12, are used for modifying the chromaticity, the shift in betatron tune in off-momentum particles.

A moderate negative chromaticity arises naturally in particle accelerators, where higher momentum particles have a smaller betatron tune. Chromaticity increases the betatron tune spread of the particle beam and consequently makes it more difficult to avoid losses due to transverse parametric resonances. The momentum aperture of the lattice, the momentum range that can be simultaneously accomodated by the lattice, is largely a product of the maximum betatron tune spread and the chromaticity.

Chromaticity also introduces Landau damping, which inhibits beam instabilities due to collective effects. For the Recycler in particular, chromaticity is necessary to prevent a beam instability in which adjacent high-intensity particle bunches couple to each other through long-range relativistic Coulomb forces (see [42]). Besides chromaticity, collective effects are mitigated in the Main Injector and Recycler by devices known as dampers [43]. Damper 
systems measure coherent beam oscillations and respond by deflecting the beam to cancel the oscillation. A proposal to introduce octopoles in the Recycler for Landau damping was found to be marginally effective [44].

Recently, an electron cloud instability was observed in the Fermilab Recycler that was beyond the capability of either the chromaticity or the damper to prevent and delayed commissioning of the Recycler [15,45-47]. Electron cloud instabilities depend critically on the surface of the accelerator beampipe, which improved over time in a process known as beampipe conditioning [48]. Due to beampipe conditioning, the electron cloud instability abated and the Recycler was able to resume commissioning. However, we expect to encounter this electron cloud instability again when we increase the bunch intensity as part of the PIP-II upgrade. If high-intensity beampipe conditioning does not abate the electron cloud formation again, a digital damper upgrade can be considered to address the instability. Our research found that the distribution of batch intensities impacted the instability and also that the instability forms as a consequence of the gradient dipoles in the Recycler. The Recycler electron instability is a subject of active research which I contributed to directly $[45,46]$ but it is beyond the scope of this dissertation.

When slip-stacking in the Recycler, recall there are two beams with differing average momenta. The momentum aperture of the Recycler and Main Injector must accommodate the entire momentum range of both beams. In addition, the higher momentum bunches and lower momentum bunches must be captured in pairs by large buckets generated by the Main Injector RF. During slip-stacking in the Recycler each bunch is maintained by RF cavities no greater than $100 \mathrm{kV}$ and after slip-stacking those bunches are captured in the Main Injector using a total RF cavity voltage of $600 \mathrm{kV}$. Table 2.3 shows the combination of changes in RF voltage and frequency used to capture the beam in the Main Injector and accelerate it to $120 \mathrm{GeV}$. The Main Injector is operated with $18 \mathrm{RF}$ cavities with that can 


\begin{tabular}{|l|l|l|l|}
\hline $\mathrm{t}(\mathrm{s})$ & $\mathrm{p}(\mathrm{MeV} / \mathrm{c})$ & $\phi_{s}$ & $\mathrm{RF}(\mathrm{MV})$ \\
\hline 0.05 & 8.830 & $0.000^{\circ}$ & 0.6 \\
0.08 & 8.830 & $0.000^{\circ}$ & 2.2 \\
0.13 & 9.010 & $2.442^{\circ}$ & 2.6 \\
0.15 & 9.360 & $4.079^{\circ}$ & 2.8 \\
0.18 & 10.25 & $10.26^{\circ}$ & 3.9 \\
0.23 & 16.25 & $25.81^{\circ}$ & 4.5 \\
0.28 & 27.37 & $36.12^{\circ}$ & 4.5 \\
0.30 & 32.16 & $36.05^{\circ}$ & 4.5 \\
\hline
\end{tabular}

\begin{tabular}{|l|l|l|l|}
\hline $\mathrm{t}(\mathrm{s})$ & $\mathrm{p}(\mathrm{MeV} / \mathrm{c})$ & $\phi_{s}$ & $\mathrm{RF}(\mathrm{MV})$ \\
\hline 0.33 & 39.33 & $35.96^{\circ}$ & 4.5 \\
0.38 & 51.23 & $35.79^{\circ}$ & 4.5 \\
0.45 & 67.83 & $35.56^{\circ}$ & 4.5 \\
0.50 & 79.60 & $35.40^{\circ}$ & 4.5 \\
0.60 & 102.7 & $33.57^{\circ}$ & 4.5 \\
0.64 & 111.5 & $29.85^{\circ}$ & 4.5 \\
0.67 & 116.5 & $18.23^{\circ}$ & 4.5 \\
0.73 & 119.7 & $0.000^{\circ}$ & 4.5 \\
\hline
\end{tabular}

Table 2.3: The table indicates time into the Main Injector cycle, expected momentum of the beam, synchronous phase, and total Main Injector RF voltage.

provide a total maximum RF voltage of $4 \mathrm{MV}$.

\section{$2.5 \quad$ PIP-II \& PIP-III}

PIP-II is a planned accelerator upgrade to increase the $120 \mathrm{GeV}$ proton beam power from $700 \mathrm{~kW}$ to $1.2 \mathrm{MW}$ in support of present and future $120 \mathrm{GeV}$ neutrino experiments $[7,49]$. A major feature of the the PIP-II upgrade is a new $800 \mathrm{MeV}$ superconducting Linac to replace the current $400 \mathrm{MeV}$ Linac. Fig. 2.13 shows the siting of the new Linac. The increase in injection energy into the Booster allows the bunch intensity in the Booster to increase by $50 \%$, because the relativistic motion slows down the Coulomb forces from the higher charge. An important manifestation of the relativistic space-charge forces is the incoherent betatron tune-shift, given by:

$$
\nu_{\text {incoh }}=-\frac{\pi \lambda r_{o} R}{2 \epsilon_{N} \beta \gamma^{2}} \sim-\frac{N}{\beta \gamma^{2}}
$$

The energy increase and the intensity increase actually results in a net $25 \%$ decrease in the incoherent betatron tune-shift and thus may decrease losses associated with transverse parametric resonances. 
To get to 1.2 MW, the PIP-II upgrade also requires shortening the Main Injector ramp time from 1.33 seconds to 1.2 seconds. With $70 \%$ higher beam power, the loss-rate of slip-stacking particles has to be reduced by at least $70 \%$ to keep nuclear activation below acceptable limits. The next three chapters will discuss the two proposals I have worked on to decrease the slip-stacking loss-rate - upgrading the Booster cycle-rate to $20 \mathrm{~Hz}$ and installing a $20 \mathrm{kV} 106 \mathrm{MHz}$ RF cavity in the Recycler.

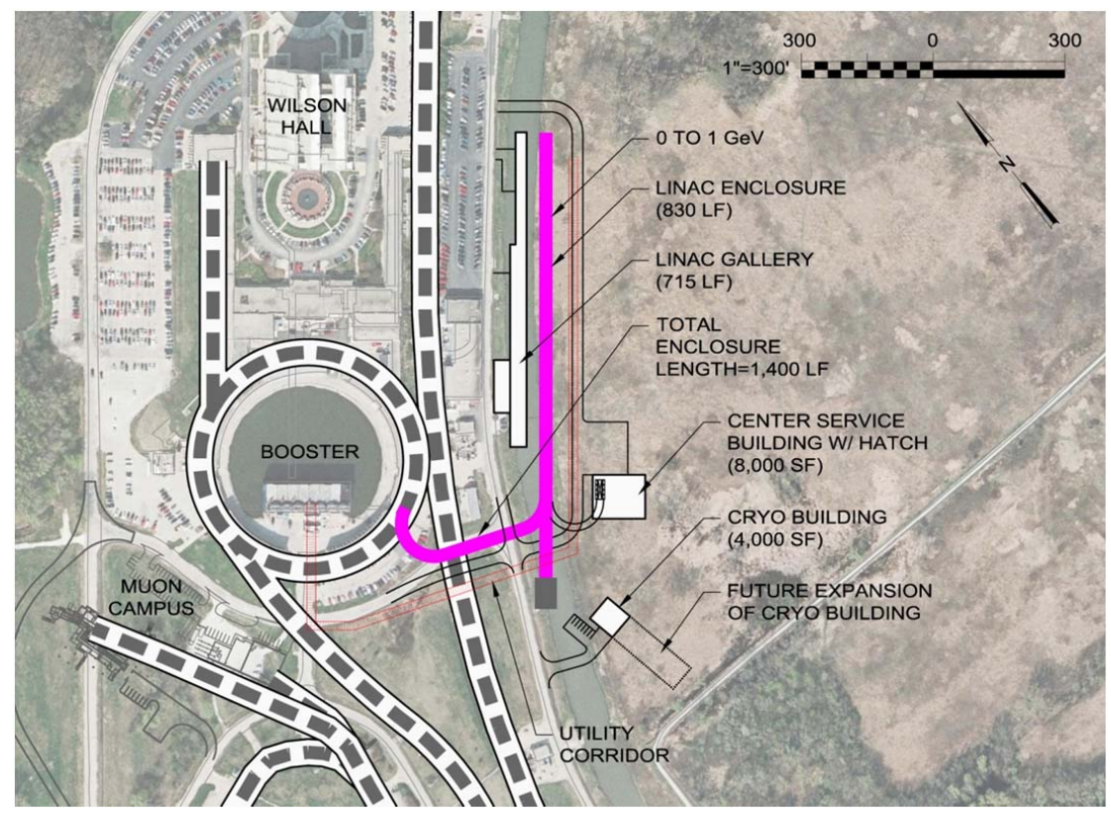

Figure 2.13: The PIP-II $800 \mathrm{MeV}$ superconducting RF Linac injects directly into the Booster and takes advantage of existing infrastructure. The Linac can be upgraded to $1 \mathrm{GeV}$ and still use the same transfer line. [7]

Any Fermilab substantial proton beam power upgrade beyond PIP-II project can be referred to as PIP-III [50]. The primary justification for the PIP-III upgrade is the Deep Underground Neutrino Experiment (DUNE) [49,51], formerly referred to as the Long Baseline Neutrino Experiment (LBNE). DUNE would measure matter-antimatter asymmetry in the neutrino sector, neutrino mass hierarchy, and neutrino oscillation parameters to great precision. The DUNE physics analysis grows in statistical power with the number of neu- 


\begin{tabular}{|c|c|c|c|c|}
\hline & 1st 10 years & \multicolumn{3}{|c|}{ 2nd 10 years } \\
\hline To Achieve : & 100 kT-MW-year & \multicolumn{3}{|c|}{500 kT-MW-year } \\
\hline We combine : & & Option 1 & Option 2 & Option 3 \\
\hline Mass & $10 \mathrm{kT}$ & $50 \mathrm{kT}$ & $20 \mathrm{kT}$ & $10 \mathrm{kT}$ \\
\hline Power & $1 \mathrm{MW}$ & $1 \mathrm{MW}$ & $2.5 \mathrm{MW}$ & $5 \mathrm{MW}$ \\
\hline
\end{tabular}

Table 2.4: The first phase of the experiment is for the PIP-II proton complex to deliver neutrinos to a $10 \mathrm{kT}$ DUNE detector. In the second phase, the most significant neutrino results are obtained through a detector size upgrade, a proton power upgrade, or a combination of both. The final decision will depend on a cost-benefit analysis building on the experiences of the first phase. [50]

trino events and the figure of merit is typically expressed in "kt-MW-years". As shown in Fig. 2.4, an increase in proton beam power can be used to deliver results on the same timescale with a smaller DUNE detector or results on a faster timescale with the same DUNE detector. A multi-MW neutrino program also requires innovation in high-power target design (see [52]). The high-power $120 \mathrm{GeV}$ beam could also be supplied to a high-energy muon program. For example NuStorm, a neutrino program based on stored muons, calls for a $120 \mathrm{GeV}$ proton beam $[53,54]$.

A Rapid Cycling Synchrotron (RCS) has been proposed for PIP-III to deliver a $\sim 2.4 \mathrm{MW}$ beam by replacing or upgrading the Fermilab Booster. The PIP-III RCS designs are largely classified in two types - a hybrid RCS or a smart RCS [50]. The hybrid RCS design require a 2-3 GeV Linac injection to achieve higher bunch intensity without increasing the spacecharge tune spread [55]. The smart RCS design achieves multi-MW beam power by utilizing two new technologies - an electron lens to substantially compensate the space-charge tuneshift [56] and a nonlinear magnetic insert to substantially reduce transverse parametric resonances [57]. The availability and effectiveness of these beam dynamics technologies will 
be verified in the next several years at the Fermilab Integrable Optics Test Accelerator (IOTA) $[50,58]$. An electron lens or nonlinear magnetic insert could also be applied to the Fermilab Recycler to reduce losses from betatron resonances and collective instabilities.

PIP-III RCS scenarios have not traditionally included slip-stacking in the Recycler or the Main Injector because the current slip-stacking loss rates prohibit a significantly higher beam power. However, in the coming chapters I demonstrate that slip-stacking losses can be reduced to rates compatible with multi-MW beam power. If beam from a PIP-III RCS can successful undergo slip-stacking in the Recycler, the $120 \mathrm{GeV}$ beam power under these scenarios is doubled. 


\section{CHAPTER 3}

\section{Slip-stacking Dynamics}

In this chapter, we provide a new and in-depth analysis of the dynamics of slip-stacking, a technique used to increase beam power in the Fermilab proton accelerator complex. We forgo a comprehensive simulation of all the forces on the slip-stacking beam and elect instead to focus on the single-particle dynamics unique to slip-stacking. We use this approach to analyze the effect of the Booster cycle-rate on slip-stacking. The Booster cycle-rate is currently $15 \mathrm{~Hz}$ and an upgrade to $20 \mathrm{~Hz}$ cycle-rate is proposed for the near future.

\subsection{Motivation}

Slip-stacking is integral to high-intensity operation at Fermilab and will play a central role in upgrades to the accelerator complex $[7,15,51]$. Particle loss in the slip-stacking process is a limiting factor on ultimate performance $[5,7,15]$ and the single-particle dynamics of slip-stacking are the dominant source of slip-stacking losses.

Slip-stacking was first demonstrated at the CERN SPS [59] but the emittance growth led to unacceptable particle losses. Subsequently, Fermilab has implemented slip-stacking operationally since $2004[5,60,61]$. At that time, the higher beam intensity was used to increase antiproton production for proton-antiproton collider experiments [62]. Currently, slip-stacking is applied to neutrino production for Neutrinos at Main Injector (NuMI) ex-

periments [11-13]. Slip-stacking originally took place in the Fermilab Main Injector and 
now slip-stacking is being commissioned for the Fermilab Recycler [15].

The Fermilab Proton Improvement Plan-II [7] calls for an improvement in beam power from $700 \mathrm{~kW}$ (with slip-stacking) to $1.2 \mathrm{MW}$ with an eye towards multi-MW improvements. The $70 \%$ increase in proton intensity requires a commensurate decrease in the slip-stacking loss-rate to limit activation in the tunnel. In this chapter we show that including a $20-\mathrm{Hz}$ Booster cycle-rate with the PIP-II upgrade would relax the limits on the Booster beam quality and cut particle losses due to slip-stacking. This work contributed to the decision to incorporate the $20 \mathrm{~Hz}$ Booster cycle-rate into the PIP-II proposal [63]. A 20-Hz Booster also reduces the time required to accumulate 12 batches in the Recycler, making more beam available for 8-GeV experiments [8-10]. For example, a 1.2 s Main Injector cycle time would consume $9 \mathrm{~Hz}$ of the Booster's cycles, the additional available $8-\mathrm{GeV}$ beam would increase from $6 \mathrm{~Hz}$ to $11 \mathrm{~Hz}$. Furthermore, if the Main Injector ramp cycle is shortened to extract protons at $60 \mathrm{GeV}[51]$, then a 20-Hz Booster would deposit more $60 \mathrm{GeV}$ protons on the high-power target than a 15-Hz Booster.

The analysis of slip-stacking in our work is also intended to facilitate application of slip-stacking to other accelerators and non-accelerator systems with analogous dynamics. The slip-stacking Hamiltonian is isomorphic to the driven pendulum, which has been wellstudied outside the accelerator context $[64,65]$. In general, the dynamics discussed in this chapter apply to any system governed by (nearly) identical sinusoidal potentials moving with respect to each other (or equivalently, a sinusoidal potential oscillating in amplitude). This is relevant to standing wave traps which are used in optical and acoustic physics and are instances of a controllable sinusoidal potential [66]. 


\subsection{Slip-stacking Accumulation Scheme}

Slip-stacking is a particle accelerator configuration that permits two high-energy particle beams of different momenta to use the same transverse space in a cyclic accelerator (see $[5,60])$. The two beams are longitudinally focused by two RF cavities with a small frequency difference between them. Each beam is synchronized to one RF cavity and perturbed by the other RF cavity.

During slip-stacking, the two azimuthal beam distributions are manipulated as a consequence of their difference in RF frequency. As shown in Fig. 3.1, the two beams are injected on separated portions of azimuth with a small frequency difference and overlap gradually. When the cyclic accelerator is filled and the azimuthal distribution of the two beams coincide then the two beams are accelerated together by RF cavities operating at the average frequency. The potential beam intensity of a synchrotron is doubled through the application of this technique. Injecting into unoccupied azimuthal space is necessary to prevent the injection of the incoming beam from kicking out the existing beam. Similarly, the electrostatic kicker used to extract all the batches from the storage ring requires an azimuthal gap in order to change the orbit without causing beam losses.

As Fig. 3.1 shows, the slipping rate of the buckets must be properly synchronized to the injection rate of new batches. The difference between the two RF frequencies must be equal to the product of the harmonic number of the Booster RF and the cycle rate of the Fermilab Booster. So for a Booster with a $15-\mathrm{Hz}$ cycle-rate we have a frequency separation of

$$
\Delta f=h_{B} f_{B}=84 \times 15 \mathrm{~Hz}=1260 \mathrm{~Hz}
$$

and for the proposed $20-\mathrm{Hz}$ cycle-rate we have

$$
\Delta f=h_{B} f_{B}=84 \times 20 \mathrm{~Hz}=1680 \mathrm{~Hz} .
$$




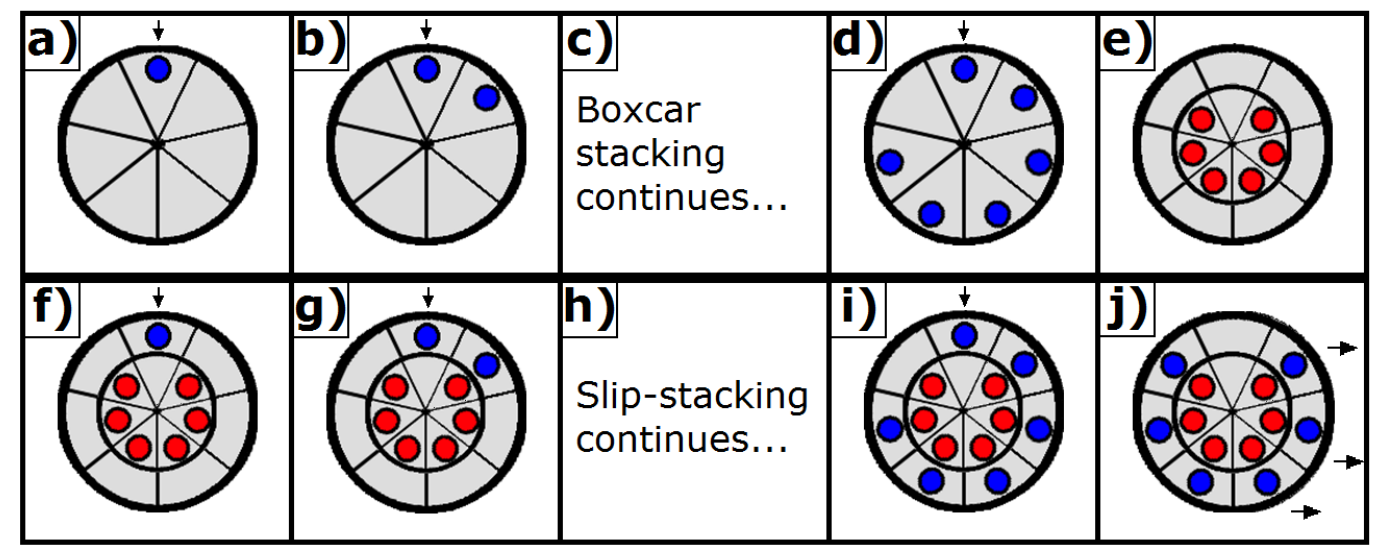

Figure 3.1: The Booster batch is represented by the circles and the Recycler (or Main Injector) is represented by the seven-sector wheel. a) The first batch is injected into the ring. b) One Booster cycle later the second batch is injected in the azimuthal space immediately behind the first batch. This is known as boxcar stacking. c, d) Boxcar stacking injections continue until six Booster batches are stored in the ring. e) The RF frequency is semiadiabatically lowered in between the sixth and seventh batch injection. f) The seventh batch is injected in the gap between the previous six batches. The two RF cavities are operating at different frequencies (slip-stacking). The first RF cavity matches the first six batches and the second RF cavity matches the next set of batches. g) One Booster cycle later the eighth batch is boxcar stacked with respect to the seventh batch and the frequency difference allows the injection to occur in the gap between the first six batches. $\mathbf{h}, \mathbf{i}$ ) Slipstacking injections continue until twelve Booster batches are stored in the ring. j) One Booster cycle later the gaps of the first six and last six batches are aligned. At that time the batches are extracted to the Main Injector (if needed) and both beams are accelerated as one. 
The difference in the frequency of the two RF cavities is related to the difference in momentum of the two beams by:

$$
\Delta \delta=\frac{\Delta f}{f_{\text {rev }} h \eta}
$$

where $h$ is the harmonic number of the Recycler and $\eta$ is the phase-slip factor of the Recycler (see Table 3.1). Consequently, the momentum difference between the two beams is $0.28 \%$ for the $15-\mathrm{Hz}$ Booster and $0.37 \%$ for the $20-\mathrm{Hz}$ Booster. The Recycler must be able to simultaneously accommodate beams in a range of momentum corresponding to the frequency difference between the RF cavities.

\subsection{Independent Buckets Approximation}

In order for particles to be accelerated after slip-stacking, particles must remain longitudinally focused into discrete bunches during slip-stacking. Fig. 3.2 shows the slip-stacking process at the level of individual bunches and RF buckets. This is an approximation however, because the dynamics of slip-stacking are explicitly time-dependent and there is no simple separatrix delineating the bucket boundary. The RF bucket is instead considered to be the region of stable phase-space area in which the beam can remain synchronized to one of the two RF cavities.

For slip-stacking, there is an RF phase associated with each of the two cavities. Without loss of generality, we adopt the frame of reference of a particle synchronized with upper RF frequency and add the influence of the lower RF cavity. If the frequency difference between the two RF cavities is $\Delta f$ then the phase of the second cavity advances at $2 \pi \Delta f t$ relative to the phase of the first cavity. We define $\omega_{\phi}=2 \pi \Delta f$, the phase-slipping frequency. The

equations of motions for a single particle under the influence of two main RF cavities are given by

$$
\dot{\phi}=2 \pi f_{\text {rev }} h \eta \delta, \dot{\delta}=f_{r e v} V_{\delta}\left[\sin (\phi)+\sin \left(\phi-\omega_{\phi} t\right)\right]
$$




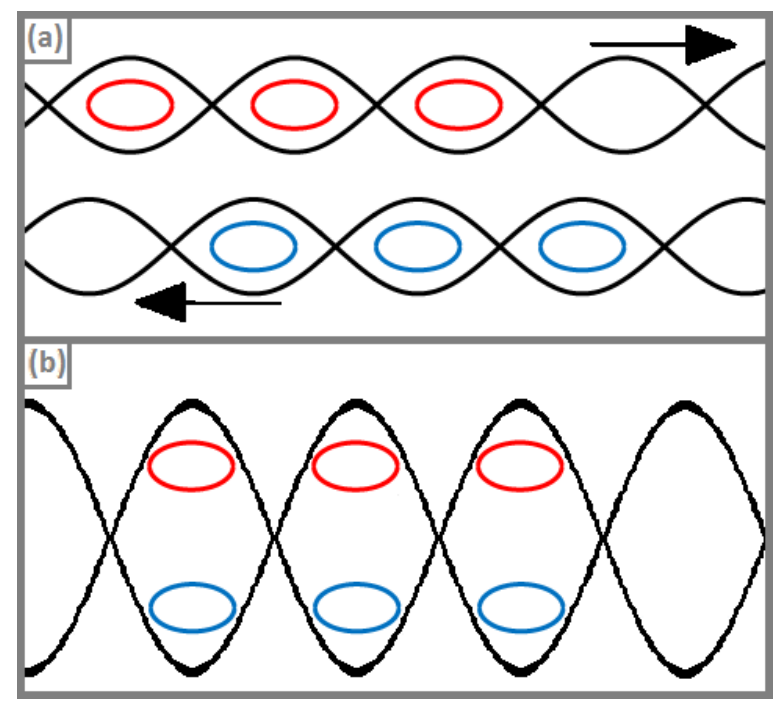

Figure 3.2: (a) Slip-stacking in the Recycler: The two beams are contained in separated RF buckets that slip past each other. (b) Capture in the Main Injector: After the two beams are aligned in the Recycler, the bunches are captured in pairs by a large RF bucket in the Main Injector.

The corresponding second-order equation of motion is

$$
\ddot{\phi}=-\omega_{s}^{2}\left[\sin (\phi)+\sin \left(\phi-\omega_{\phi} t\right)\right] .
$$

and we expand this with a trigonometic identity as

$$
\ddot{\phi}=-\omega_{s}^{2}\left[\sin (\phi)+\sin (\phi) \cos \left(\omega_{\phi} t\right)-\cos (\phi) \sin \left(\omega_{\phi} t\right)\right]
$$

In the rapid oscillation limit $\omega_{\phi} \gg \omega_{s}$ the perturbation from second RF system averages out rapidly and Eq. 3.4 approaches the single-RF system given by Eq. 1.11. Similarly, from the frame of reference of particles synchronized to the second RF system, the perturbation from the first RF system averages out rapidly. At more moderate values of $\omega_{\phi}$ relative to $\omega_{s}$, the perturbation effect complicates the motion and reduces the stable phase-space area. This effect is quantified by the slip-stacking parameter $\alpha_{s}[59,60,67]$ defined:

$$
\alpha_{s}=\frac{\omega_{\phi}}{\omega_{s}}
$$


The slip-stacking parameter can equivalently be defined in terms of the separation between the single-RF buckets relative to the bucket height. Fig. 3.3 shows the single-RF buckets and stable phase-space area for selected values of $\alpha_{s}$.

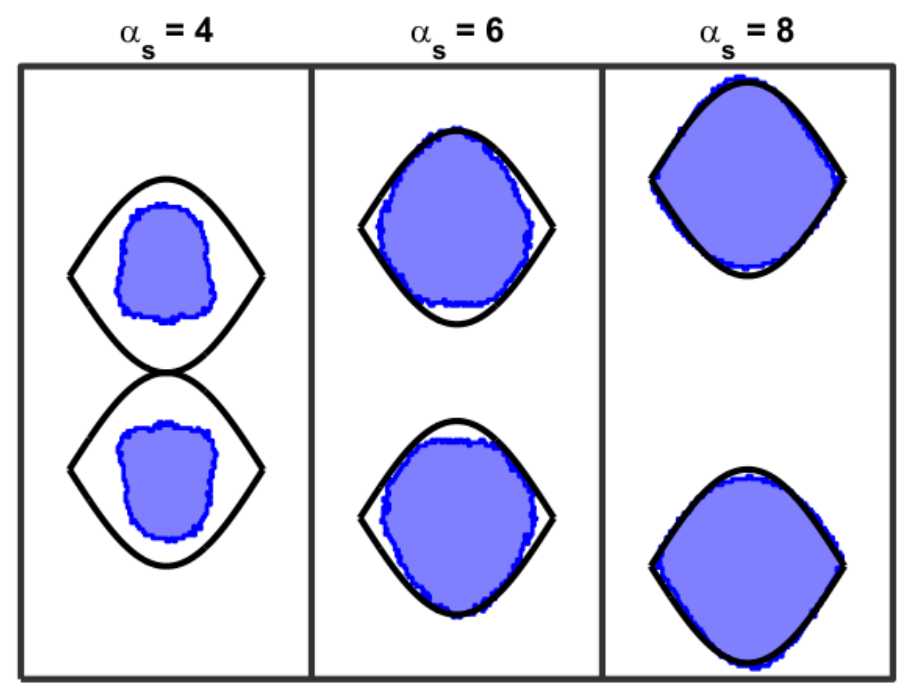

Figure 3.3: The black lines show the stable phase-space area of the single-RF bucket, an RF bucket without the perturbation from the second RF cavity. The blue lines show the stable phase-space area of the slip-stacking bucket. As the regions of phase-space separate, the stable phase-space area of the slip-stacking bucket approaches the single-RF bucket.

All nontrivial dynamics of Eq. 3.3 depend only on $\alpha_{s}$. For example, if one slip-stacking configuration has phase-slipping frequency $\omega_{\phi}$ and another configuration with the same $\alpha_{s}$ has phase-slipping frequency $\omega_{\phi}^{\prime}$ then the second phase space diagram is isomorphic to the first where the $\delta$ axis must be scaled by $\omega_{\phi}^{\prime} / \omega_{\phi}$.

\subsection{General Perturbative Solution}

Eq. 3.4 can be expanded into powers of $\phi$ to consider the small $\phi$ perturbation:

$$
\ddot{\phi}=-\omega_{s}^{2}\left\{\sum_{k=0}^{\infty} \frac{(-1)^{k}}{(2 k+1) !} \phi^{2 k+1}\left[1+\cos \left(\omega_{\phi} t\right)\right]-\sum_{k=0}^{\infty} \frac{(-1)^{k}}{(2 k) !} \phi^{2 k} \sin \left(\omega_{\phi} t\right)\right\} .
$$

We use the Poincare-Lindstedt method (see Ch. 2 of [3]) to find the perturbative solution 
to Eq. 3.6 as a linear combination of oscillatory terms. We substitute the case $\phi=0$ into Eq. 3.6 and we solve to generate $\phi=-\alpha_{s}^{2} \sin \left(\omega_{\phi} t\right)$. Next we use $\phi=-\alpha_{s}^{2} \sin \left(\omega_{\phi} t\right)$ to generate $A_{n} \sin \left(n \omega_{\phi} t\right)$ terms. The coefficients $A_{n}$ are of the order $\alpha_{s}^{-2 n}$ and do not depend on the initial coordinates of the particle. These terms form the particular solution:

$$
\phi_{p}=\sum_{n=1}^{\infty} A_{n} \sin \left(n \omega_{\phi} t\right) .
$$

The frequency spectrum of the particular solution depends only on harmonics of $\omega_{\phi}$ and therefore returns to the same point every phase-slipping period $T_{\phi}$. The particular solution is a fixed point in the Poincaré map but corresponds to a moving bucket center in real time. General trajectories perform synchrotron oscillations around this moving bucket center, as shown in Fig. 3.4. Poincaré maps for other values of $\alpha_{s}$ are given in Appendix A.

We continue the perturbation to the general case by using $\phi=\phi_{p}+\rho \sin \left(\omega_{s} t+\psi\right)$, the sum of the particular solution (Eq. 3.7) and the rapid-oscillation solution (Eq. 1.13). Using $\phi=\phi_{p}+\rho \sin \left(\omega_{s} t+\psi\right)$ generates terms of the form $B_{m, n} \sin \left[m(1+\sigma) \omega_{s} t+n \omega_{\phi} t+m \psi\right]$. The shift in the synchrotron oscillation frequency $\sigma$ is a necessary contribution to the coefficient of $\sin \left[(1+\sigma) \omega_{s} t+\psi\right]$ in Eq. 3.6 to counterbalance the contribution made by the crossmultiplication of higher order $B_{m, n} \sin \left[m(1+\sigma) \omega_{s} t+n \omega_{\phi} t+m \psi\right]$ terms. For any integer $m>0$ and any integer $n$, the coefficients $B_{m, n}$ are of the order $\rho^{m} \alpha_{s}^{-2|n|}$; except when $m$ is even and $n=0$, in which case the coefficients $B_{m, 0}$ are of the order $\rho^{m} \alpha_{s}^{-2}$. Writing out the full perturbative solution, we have:

$$
\begin{aligned}
\phi=\sum_{n=1}^{\infty} A_{n} \sin \left(n \omega_{\phi} t\right) & +\sum_{m=1}^{\infty} \sum_{n=1}^{\infty} B_{m,+n} \sin \left[m(1+\sigma) \omega_{s} t+n \omega_{\phi} t+m \psi\right] \\
& +\sum_{m=1}^{\infty} \sum_{n=1}^{\infty} B_{m,-n} \sin \left[m(1+\sigma) \omega_{s} t-n \omega_{\phi} t+m \psi\right] .
\end{aligned}
$$

The trajectory of a particle in a slip-stacking RF bucket is referred to as a (purely) rotating solution in the driven-pendulum literature. The particular solution was previously obtained by Zhang and Ma [68]. An alternate perturbative approach for the general solution in 

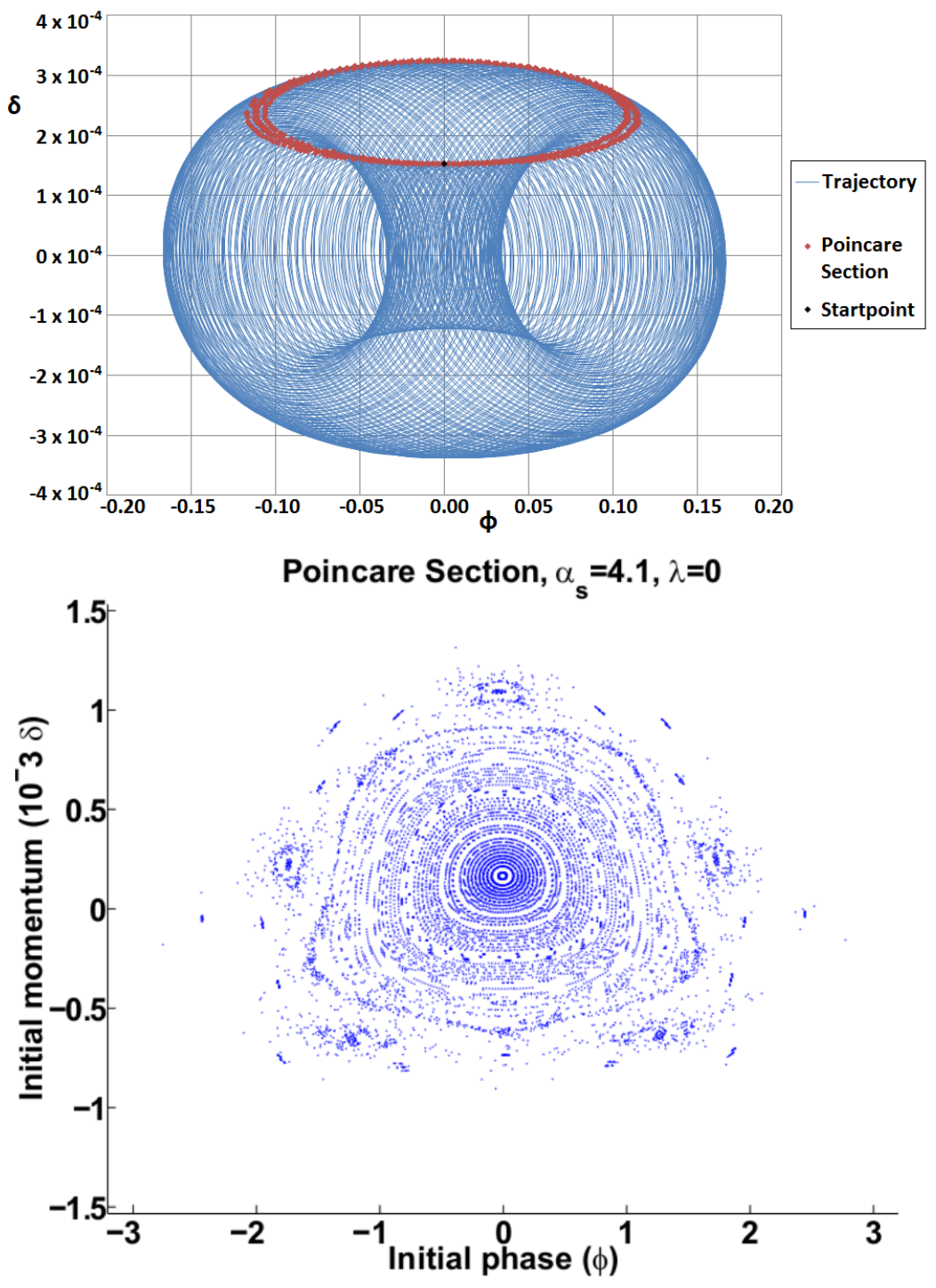

Figure 3.4: (top) A phase-space trajectory (blue) of a slip-stacking particle near the center of its RF bucket. The motion primarily consists of two oscillations, one at the synchrotron frequency and one at the phase-slipping frequency. A Poincaré section of this trajectory, the coordinates of the particle once every phase-slipping period, is shown in red. (bottom) Poincaré sections for several phase-space trajectories. The fourth-order and fifth-order resonances are clearly seen. 
implicit form is given in [69]. We are first to find a general and explicit solution.

The perturbative solution for the small oscillations around the moving bucket center can be expressed in coefficients up to order $\alpha_{s}^{-4}$ and $\rho \alpha_{s}^{-2}$. The derivation shown in Appendix B leads to the equations of motion:

$$
\begin{aligned}
\phi= & A_{1} \sin \left(\omega_{\phi} t\right)+A_{2} \sin \left(2 \omega_{\phi} t\right) \\
& +\rho \sin \left[(1+\sigma) \omega_{s} t+\psi\right] \\
& +B_{1,1} \sin \left[(1+\sigma) \omega_{s} t+\omega_{\phi} t+\psi\right]+B_{1,-1} \sin \left[(1+\sigma) \omega_{s} t-\omega_{\phi} t+\psi\right] . \\
\delta= & \frac{1}{2 \pi f_{\text {rev }} h \eta} \dot{\phi} . \\
A_{1}= & -\frac{1}{\alpha_{s}^{2}-1} . \\
A_{2}= & \frac{1}{\left(2 \alpha_{s}\right)^{2}-1}\left(\frac{A_{1}}{2}\right) . \\
B_{1, \pm 1}= & \frac{\alpha_{s}^{-1}}{\alpha_{s} \pm 2}\left(\frac{\rho}{2}\right) . \\
\sigma= & \frac{3}{4} \alpha_{s}^{-4} .
\end{aligned}
$$

The parameters $\rho$ and $\psi$ are determined by initial conditions, shown explicitly in Appendix B. We are the first to discover and calculate the dependence of the synchrotron frequency shift $\sigma$ on the slip-stacking perturbation.

Substituting the perturbative terms from Eq. 3.8 into Eq. 3.6 indicates that a new parametric resonance will occur wherever

$$
m \omega_{s}(1+\sigma)=m \omega_{s}^{\prime}=n \omega_{\phi}
$$

For example, the $\rho^{m} \alpha_{s}^{-2(n-1)} \sin \left[m(1+\sigma) \omega t-(n-1) \omega_{\phi} t+m \psi\right]$ term will be multiplied by $\cos \left(\omega_{\phi} t\right)$ in Eq. 3.6 and lead to an uncontrolled growth term $\ddot{\phi} \propto \rho^{m} \alpha_{s}^{-2(n-1)} \sin (m \psi)$. The Poincaré map in Fig. 3.4 reveals the resonant behavior. The case where $m \omega_{s}=\omega_{\phi}$ was previously investigated by Mills [67]. An analytical description of the stable phase-space boundary (the largest invariant circle) is beyond the scope of this research. 


\subsection{Stability Maps and Area Factors}

The size and shape of slip-stacking buckets determine which portion of an injected beam distribution is lost. In application, lost particles migrate to an incorrect azimuthal location and consequently collide into the beampipe during injection, extraction, or acceleration (see next chapter). We map the stability of initial particle positions by integrating the equations of motion for each position. The integration is iterated for a sufficiently large number of revolutions (at least 30 synchrotron periods). The stability of the particle is tested after every phase-slipping period. A particle is considered lost if its phase with respect to each of the first RF cavity, the second RF cavity, and the average of the two RF cavities, is larger than a certain cut-off (we used $3 \pi / 2$ ). The remaining particles therefore belong to one of four stable regions shown in Fig. 3.5: one for the higher frequency, one for the lower frequency, one for the average frequency and average phase, and one with average frequency but $\pi$ offset from the average phase. These two stable regions at the average frequency are the original examples of dynamic stabilization [70]. Appendix $\mathrm{C}$ shows stability maps for other values of $\alpha_{s}$.

We find some slip-stacking trajectories show the features of chaotic escape $[71,72]$ and lead to particle loss only after thousands of revolutions. The stable phase-space area as a function of time is shown in Fig. 3.6 for several values of $\alpha_{s}$.

The bucket area is computed as the product of the total number of ultimately surviving points and the cell area. We define the slip-stacking area factor $F\left(\alpha_{s}\right)=\mathcal{A}_{s} / \mathcal{A}_{0}$ as the ratio of the slip-stacking bucket area to that of a single-RF bucket with the same $R F$ voltage and frequency. The area factor follows the notation of Lee (Ch. 3.II of [2]) for accelerating beams, in which the ratio of running bucket area to stationary bucket area is used. Particles in the bucket are described by Eq. 3.8 with finite coefficients and the bucket area is conserved. Consequently $F\left(\alpha_{s}\right)$ does not depend on the initial RF phase difference 


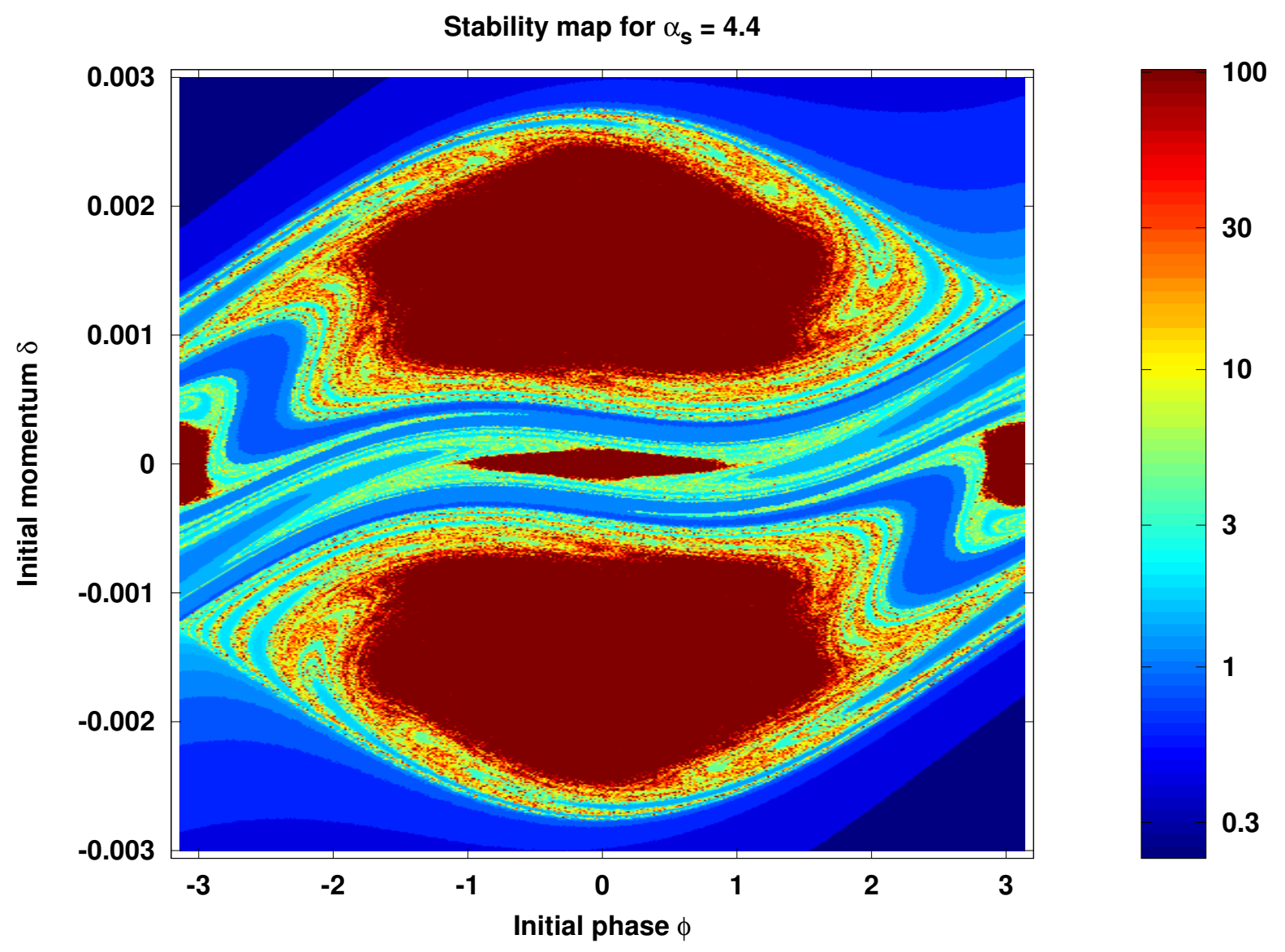

Figure 3.5: Stability of initial coordinates for $\alpha_{s}=4.4$. The color corresponds to the number of synchrotron periods a particle with the corresponding initial coordinates survives before it is lost. The two large stable regions correspond to the higher and lower RF buckets where beam is injected and maintained. The two stable regions along the $\delta_{A}=0$ axis are created by the interaction between the two RF cavities. 


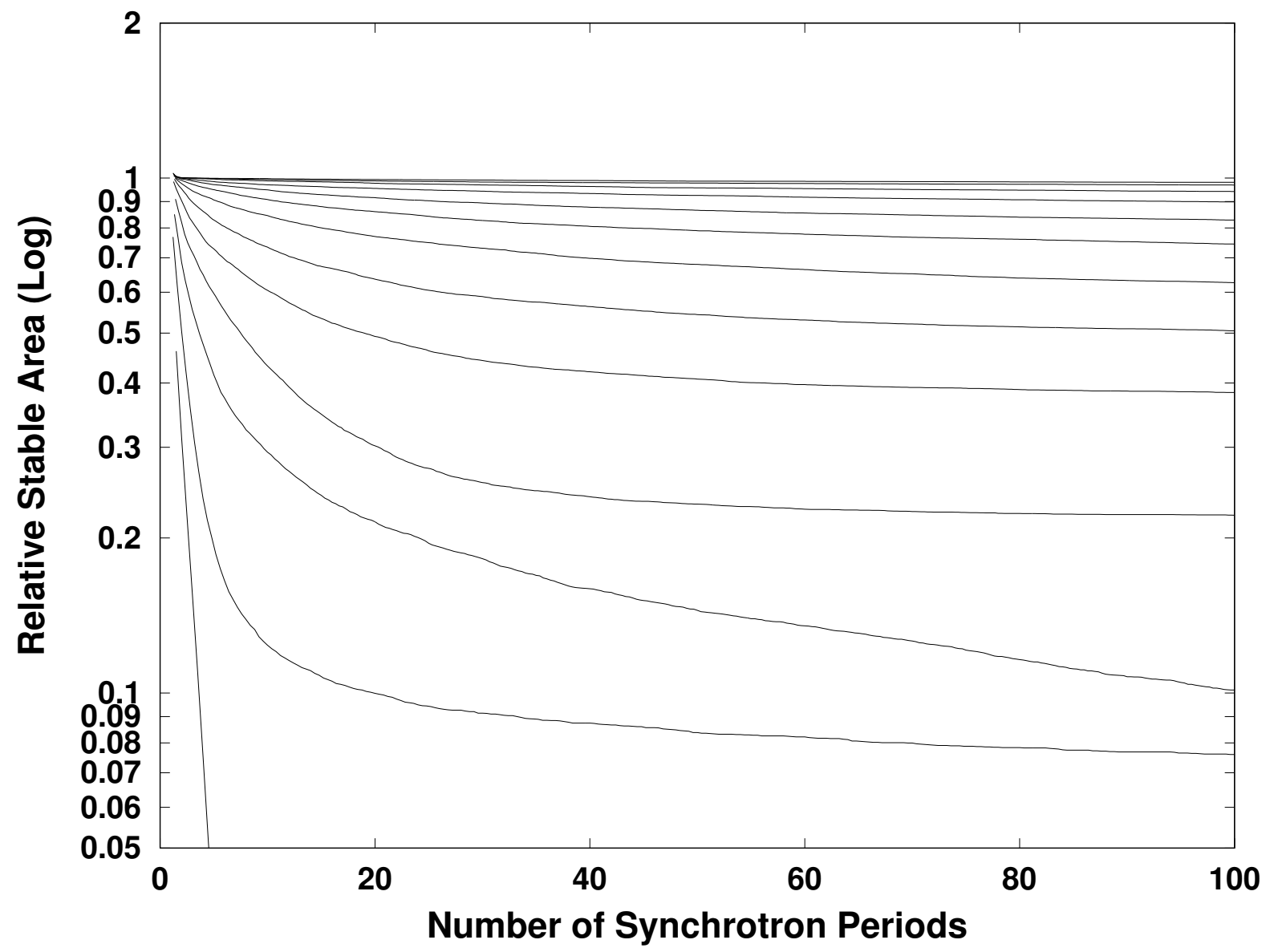

Figure 3.6: The stable area of the slip-stacking bucket relative to a single RF bucket, is plotted on a log scale and plotted over time. Each curve corresponds to a simulation with a different value of $\alpha_{s}$ with $\alpha_{s}=2.0,2.5,3.0,3.5,4.0,4.5,5.0,5.5,6.0,6.5,7.0,7.5,8.0$ (going from the bottom line to the top line). The rapid losses at the beginning corresponds to regions of phase space in which particles rapidly slip by both slip-stacking buckets. In the next phase, chaotic particle loss occurs asymptotically. 
used to generate the stability map. We write the phase space area $\left(\phi \cdot \delta\right.$ units) using $F\left(\alpha_{s}\right)$ :

$$
\mathcal{A}_{s}=\mathcal{A}_{0} F\left(\alpha_{s}\right)=\frac{16}{h|\eta|} \frac{\omega_{s}}{\omega_{\text {rev }}} F\left(\alpha_{s}\right)
$$

Fig. 3.7(a) plots the numerically derived slip-stacking area factor $F\left(\alpha_{s}\right)$. Using Fig. 3.7(a) with Eq. 3.16 provides the first method for calculating the slip-stacking stable phase-space area without requiring each case to be simulated individually. $F\left(\alpha_{s}\right)$ increases rapidly above $\alpha_{s} \approx 3$ and asymptotically approaches $1 . F\left(\alpha_{s}\right)$ has several local minimum where resonances are crossed; this loss of area occurs when large amplitude trajectories have a parametric resonance and therefore does not occur at precise integer values of $\alpha_{s}$.

In application, slip-stacking is tuned to maximize stable phase-space area while holding $\omega_{\phi}$ constant. The value of $\omega_{\phi}$ is generally constrained by gross features of the accelerators, for example the harmonic number and cycle time. The slip-stacking parameter $\alpha_{s}$ is tuned through changing $\omega_{s}$ which is proportional to the square root of the applied RF voltage. Furthermore $\omega_{s}$ changes the bucket area by both the slip-stacking area factor $F\left(\alpha_{s}\right)$ and the single-RF bucket area, so there is an optimal voltage in which phase space area is maximized. We absorb the dependence on $\omega_{s}$ in Eq. 3.16 by defining the modified slipstacking area factor $Z\left(\alpha_{s}, \lambda\right)$ :

$$
\mathcal{A}_{s}=\frac{16}{h|\eta|} \frac{\omega_{\phi}}{\omega_{\text {rev }}}\left(\frac{F\left(\alpha_{s}\right)}{\alpha_{s}}\right)=\frac{16}{h|\eta|} \frac{\omega_{\phi}}{\omega_{\text {rev }}} Z\left(\alpha_{s}\right)
$$

This modified slip-stacking area factor $Z$ is proportional to the slip-stacking phase-space area with a coefficient independent of voltage. The modified area factor $Z\left(\alpha_{s}\right)$ is graphed in Fig. 3.7(b). $Z\left(\alpha_{s}\right)$ is maximal near $\alpha_{s}=6.2$ and when considering other optimization criteria 5.5 to 7 is a practical tuning range for $\alpha_{s}$. 

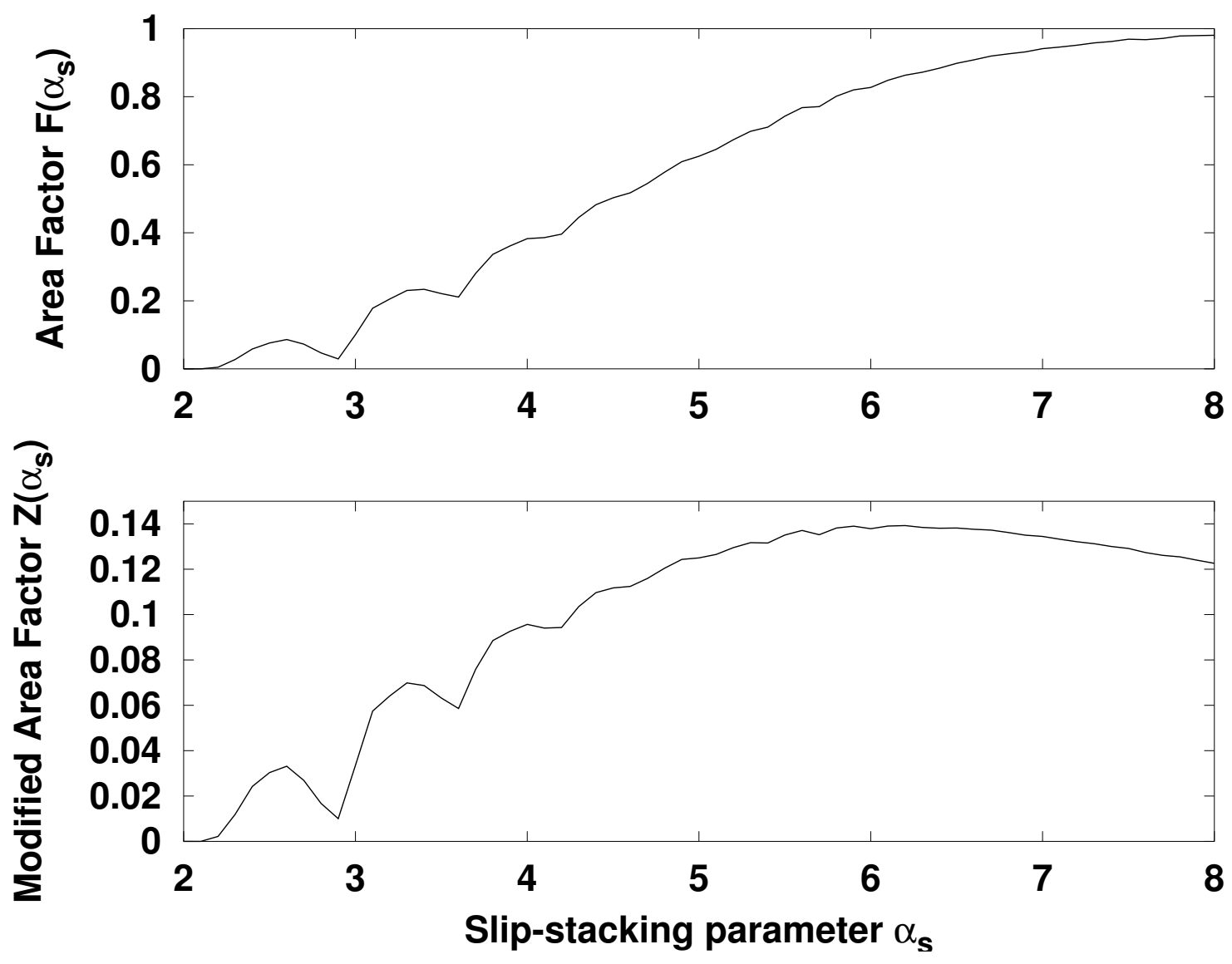

Figure 3.7: (a) The slip-stacking area factor as a function of $\alpha_{s}$. As $\alpha_{s}$ increases the distance between the RF buckets becomes greater, the buckets become more independent, and the slip-stacking bucket area approaches the single-RF bucket area. (b) The modified slip-stacking area factor as a function of $\alpha_{s}$. The modified slip-stacking area factor is maximized near $\alpha_{s}=6.2$. 


\subsection{Injection Efficiency of Gaussian Beams}

The stability maps can also be used to analyze injection scenarios, by weighting the (scaled) stability maps according to a distribution that represents the number of incoming particles injected into that region of phase-space. We used this technique to identify the $97 \%$ admittance, the greatest longitudinal emittance an incoming gaussian-distributed beam could have and still achieve $97 \%$ injection efficiency at its optimal value of $\alpha_{s}$. The $97 \%$ longitudinal beam emittance is given in Eq. 3.18 below:

$$
\epsilon=\pi \sigma_{p} \sigma_{T}, \epsilon_{97 \%}=2.17^{2} \pi \sigma_{p} \sigma_{T}
$$

The current accelerator upgrade proposal, Proton Improvement Plan II (PIP-II) [7], defines a minimum $97 \%$ slip-stacking efficiency required to maintain current loss levels while increasing intensity. Fig. 3.8 shows the $97 \%$ longitudinal admittance as a function of aspect ratio and demonstrates the consequences of a mismatched injection into a slip-stacking bucket. Fig. 3.9 shows the optimal value of $\alpha_{s}$ as a function of aspect ratio. The optimal value of $\alpha_{s}$ determines the optimal RF cavity voltage, shown in Fig. 3.10. These results were obtaining using parameter values specific to slip-stacking in the Fermilab Recycler (see Table. 3.1).

A measured value for the Booster emittance is $0.17 \mathrm{eV} \cdot \mathrm{s}$ (see next chapter). The Fermilab Booster uses bunch rotation via quadrupole excitation $[35,36]$, with parameters that are actively tuned to minimize losses. With bunch rotation, the aspect ratio is measured to be $1.45 \mathrm{MeV} / \mathrm{ns}$ at extraction from the Booster (see next chapter). At Recycler rf cavity voltage $V_{0}=100 \mathrm{kV}$, the slip-stacking parameter for the Recycler is $\alpha_{s}\left(V_{0}\right) \approx 4.39$ for a 15 $\mathrm{Hz}$ Booster cycle-rate and $\alpha_{s}\left(V_{0}\right) \approx 5.86$ for a $20-\mathrm{Hz}$ Booster cycle-rate. For other voltages, the Recycler slip-stacking parameter is given by $\alpha_{s}(V)=\alpha_{s}\left(V_{0}\right) \sqrt{V / V_{0}}$.

We examine the $97 \%$ efficiency benchmark for both a 15-Hz Booster cycle-rate and a 20- 


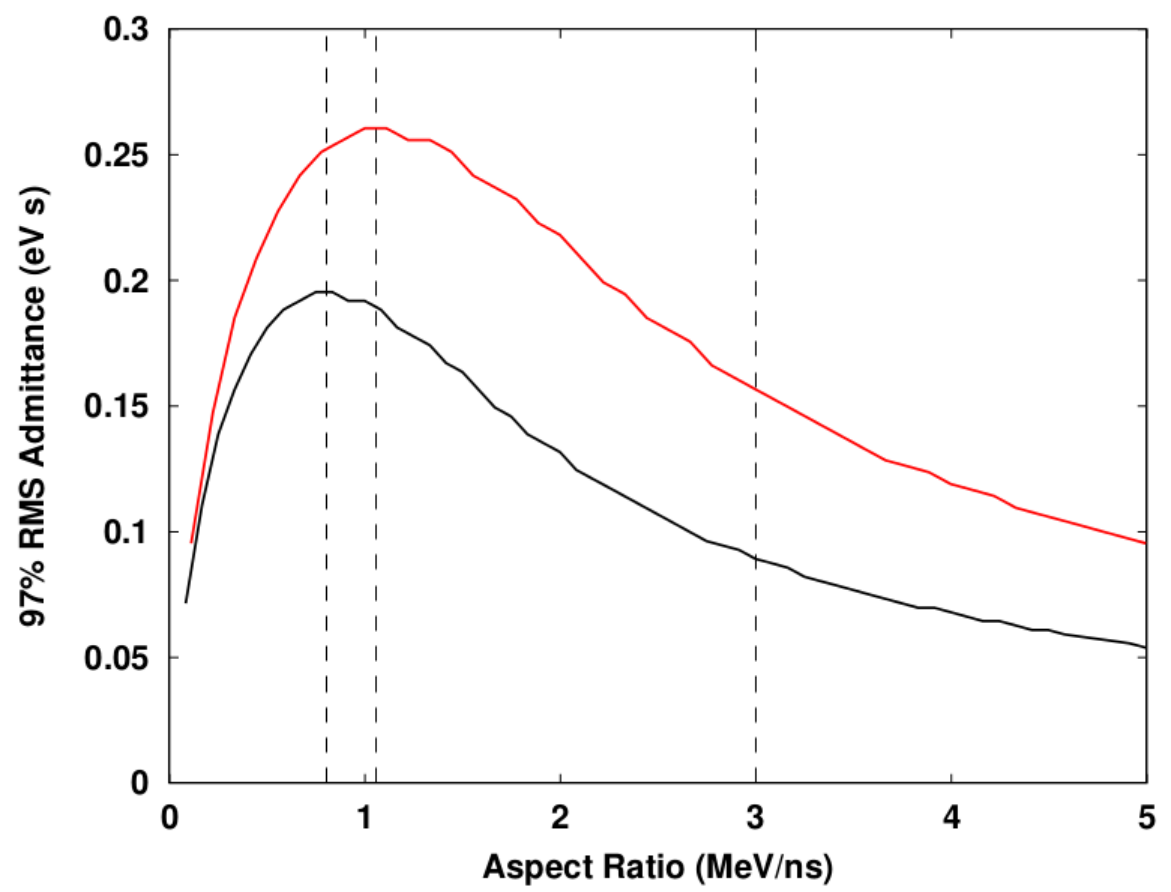

Figure 3.8: The $97 \%$ admittance at $97 \%$ efficiency (at an optimal value of $\alpha_{s}$ ) is shown as a function of aspect ratio. The bottom line (black) is for the $15-\mathrm{Hz}$ Booster cycle-rate (status quo) and top line (red) is for 20-Hz Booster cycle-rate (PIP-II). 


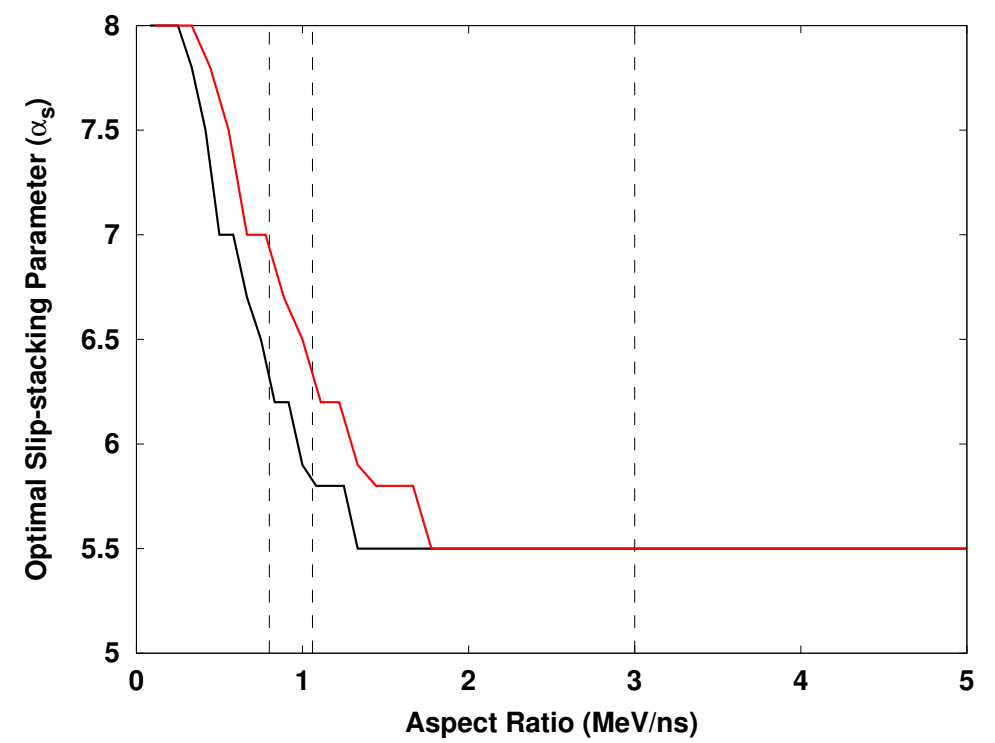

Figure 3.9: The optimal slip-stacking parameter $\alpha_{s}$ for maximum emittance (at $97 \%$ efficiency) is shown as a function of aspect ratio. Values of $\alpha_{s}$ greater than 8 are not evaluated. The bottom line (black) is for the $15-\mathrm{Hz}$ Booster cycle-rate (status quo) and top line (red) is for $20-\mathrm{Hz}$ Booster cycle-rate (PIP-II).

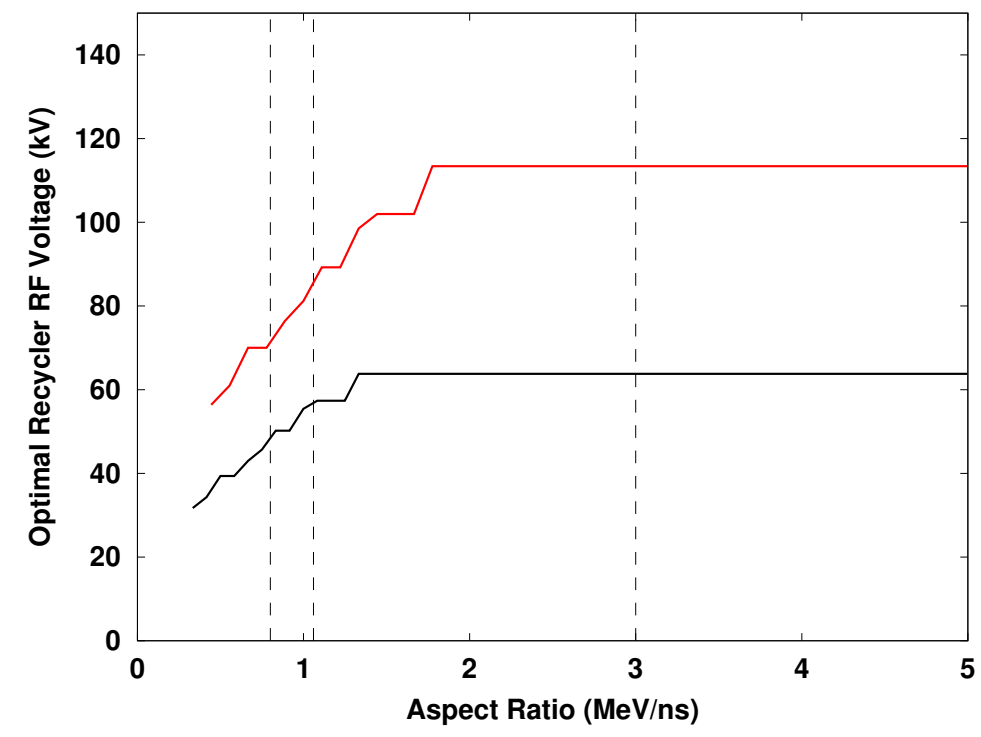

Figure 3.10: The optimal Recycler RF voltage for maximum emittance (at $97 \%$ efficiency) is shown as a function of aspect ratio. The bottom line (black) is for the $15-\mathrm{Hz}$ Booster cycle-rate (status quo) and top line (red) is for 20-Hz Booster cycle-rate (PIP-II). 


\begin{tabular}{|l|l|}
\hline Recycler Kinetic Energy $(E)$ & $8 \mathrm{GeV}$ \\
Recycler Reference RF freq. $(f)$ & $52.8 \mathrm{MHz}$ \\
Recycler Harmonic number $(h)$ & 588 \\
Recycler Phase-slip factor $(\eta)$ & $-8.6^{*} 10^{-3}$ \\
Maximum Recycler RF Voltage $(V)$ & $2 \times 150 \mathrm{kV}$ \\
Booster harmonic number $\left(h_{B}\right)$ & 84 \\
Booster cycle rate $\left(f_{B}\right)$ & $15 / 20 \mathrm{~Hz}$ \\
Difference in Recycler RF freq. $(\Delta f)$ & $1260 / 1680 \mathrm{~Hz}$ \\
\hline Measured Booster emittance $\left(\epsilon_{97 \%}\right)$ & $0.17 \mathrm{eV} \cdot \mathrm{s}$ \\
Nominal Booster Aspect Ratio & $3.00 \mathrm{MeV} / \mathrm{ns}$ \\
Rotated Booster Aspect Ratio & $1.45 \mathrm{MeV} / \mathrm{ns}$ \\
Nominal Recycler Aspect Ratio $(100 \mathrm{kV})$ & $1.06 \mathrm{MeV} / \mathrm{ns}$ \\
Nominal Recycler Aspect Ratio $(57 \mathrm{kV})$ & $0.80 \mathrm{MeV} / \mathrm{ns}$ \\
\hline
\end{tabular}

Table 3.1: Recycler and Booster parameters used in analysis.

$\mathrm{Hz}$ Booster cycle-rate. A 20-Hz Booster cycle-rate increases the RF frequency separation by a factor of $4 / 3$ and therefore permits a $4 / 3$ higher bucket height (for the same level of bucket independence $\alpha_{s}$ ). Consequently a 20-Hz Booster cycle-rate permits operation with either a significantly greater Booster admittance or injection efficiency. Fig. 3.11 superimposes a Booster beam injection (3 MeV/ns) for a $15-\mathrm{Hz}$ Booster slip-stacking bucket and $20-\mathrm{Hz}$ Booster slip-stacking bucket. For a beam with an injected aspect ratio of $2.00 \mathrm{MeV} / \mathrm{ns}$ the $97 \%$ admittance is $0.132 \mathrm{eV} \cdot \mathrm{s}$ for a $15-\mathrm{Hz}$ Booster cycle-rate and $0.218 \mathrm{eV} \cdot \mathrm{s}$ for a $20-\mathrm{Hz}$ Booster cycle-rate. Table 3.2 shows the improvement from a 20-Hz Booster cycle-rate as greater efficiency. A 20-Hz Booster cycle-rate is clearly superior for high-intensity operation.

The scaling symmetry used to analyze the 20-Hz Booster cycle-rate can generalized. An optimization at phase-slipping frequency $\omega_{\phi}$ and aspect ratio $r$ is equivalent to an optimization at phase-slipping frequency $\omega_{\phi}^{\prime}$ and aspect ratio $\left(\omega_{\phi}^{\prime} / \omega_{\phi}\right) r$. The same optimal slip-stacking parameter would be obtained at a higher synchrotron frequency $\left(\omega_{\phi}^{\prime} / \omega_{\phi}\right) \omega_{s}$, 


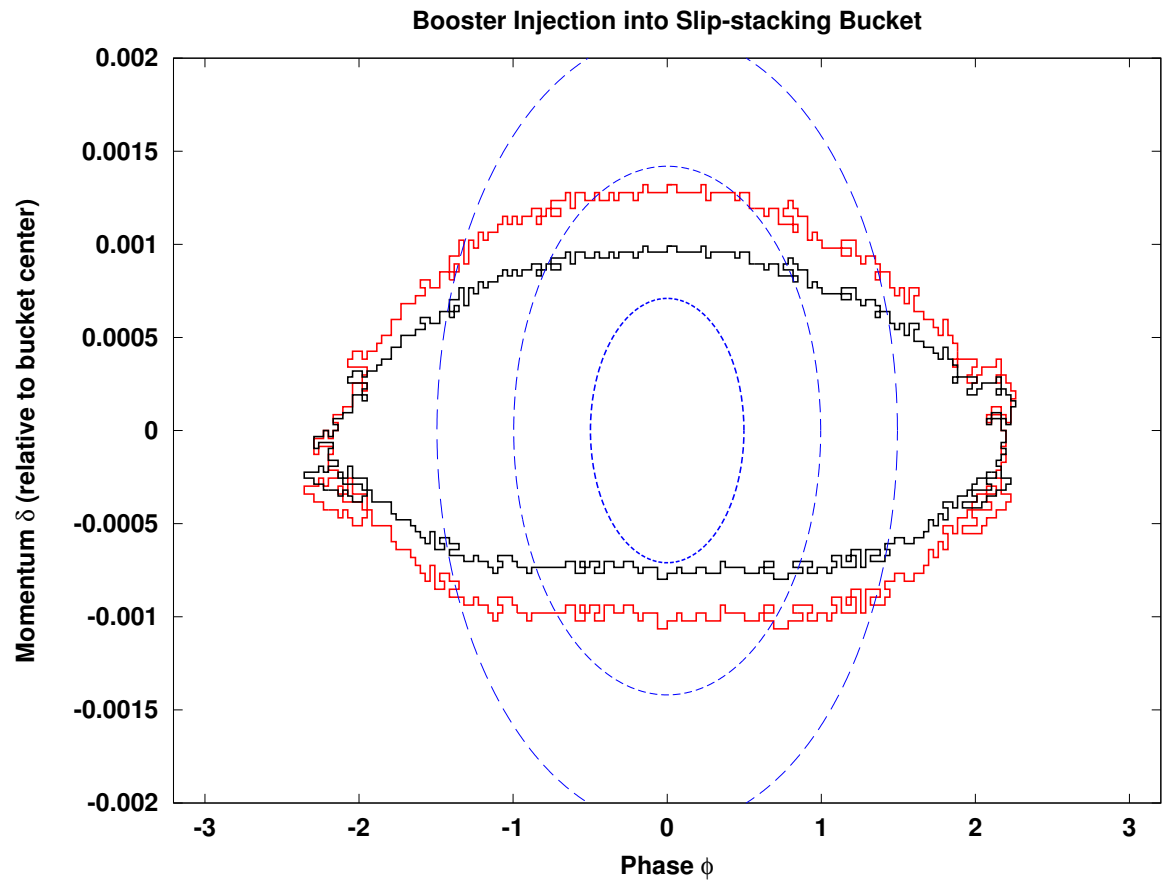

Figure 3.11: The shape of the slip-stacking Bucket is shown in black for the case of the $15-\mathrm{Hz}$ Booster and in red for the case of the 20-Hz Booster. Both slip-stacking buckets are calculated for $\alpha_{s}=5.5$ and optimized for bucket height. The three dashed blue lines represent $1 \sigma, 2 \sigma$, and $3 \sigma$ of a Gaussian distribution representing a typical Booster injection. In this case, the beam emittance is $0.1 \mathrm{eV} \cdot \mathrm{s}$ and the aspect ratio is $3 \mathrm{MeV} / \mathrm{ns}$.

\begin{tabular}{|l|r|c|}
\hline Losses & $15 \mathrm{~Hz}$ & $20 \mathrm{~Hz}$ \\
\hline with $2.00 \mathrm{MeV} / \mathrm{ns} \mathrm{\&} 0.1 \mathrm{eV} \cdot \mathrm{s}$ & $\mathbf{1 . 3 6} \%$ & $\mathbf{0 . 1 4} \%$ \\
with $2.00 \mathrm{MeV} / \mathrm{ns} \mathrm{\&} 0.12 \mathrm{eV} \cdot \mathrm{s}$ & $\mathbf{2 . 3 9} \%$ & $\mathbf{0 . 3 3} \%$ \\
with $2.00 \mathrm{MeV} / \mathrm{ns} \& 0.18 \mathrm{eV} \cdot \mathrm{s}$ & $6.58 \%$ & $\mathbf{1 . 6 7} \%$ \\
\hline
\end{tabular}

Table 3.2: Holding aspect ratio and emittance constant, the slip-stacking losses are dramatically reduced in a $20-\mathrm{Hz}$ Booster. Bolded values pass the $97 \%$ efficiency benchmark. 
increasing the rf voltage at $\left(\omega_{\phi}^{\prime} / \omega_{\phi}\right) r$ to $\left(\omega_{\phi}^{\prime} / \omega_{\phi}\right)^{2} V$.

\subsection{Recycler Slip-stacking Constraints}

In this section we consider two additional types of hardware constraints on slip-stacking. The first constraint is on the performance of RF cavities. These include beam-loading effects, RF voltage constraints, and RF power constraints. The second constraint is momentum aperture, the range of beam momentum that can be stored in the storage ring lattice. The constraints depend on the specific RF cavity and magnet hardware installed in the Recycler.

Beam-loading effects impact the effectiveness of slip-stacking and were addressed in the Main Injector by the development of a beam-loading compensation system with $-14 \mathrm{~dB}$ feedback and -20dB feedforward [73-75]. The beam-loading effects on slip-stacking in the Recycler will be an order of magnitude weaker than in the Main Injector and can be compensated if necessary. The typical beam-loading voltage is $\sim 2 \mathrm{kV}[74]$ compared to a typical $\mathrm{RF}$ cavity voltage of $90 \mathrm{kV}[75]$. In the Main Injector the $R_{s h} / Q$ of the $\mathrm{RF}$ cavities is $100 \Omega$ [74], while in the Recycler the $R_{s h} / Q$ is $13 \Omega$ [76]. Slip-stacking loss studies (see next chapter) indicate the losses are not dominated by intensity-dependent effects.

The gains in slip-stacking efficiency under the $20-\mathrm{Hz}$ Booster scenario also require an increase in the operating voltage of the RF cavities (see Fig. 3.10). The Recycler RF cavities are designed for $150 \mathrm{kV}$ maximum voltage [76] but operational experience has shown that reliability suffers at voltages above $100 \mathrm{kV}$. If we increase the RF cavity voltage from 64 $\mathrm{kV}$ to $100 \mathrm{kV}$, this is a factor of $\sim 1.56$. The duty factor may also decrease (by no more than $3 / 4$ ) in the case of a $20-\mathrm{Hz}$ Booster; the power dissipation would increase by at least $(3 / 4)(100 / 64)^{2} \approx 1.83$. The maximum Recycle RF power is $150 \mathrm{~kW}$, preparation for a $20-\mathrm{Hz}$ Booster should include the possibility of overheating in the Recycler RF cavities. 


\begin{tabular}{|l|c|c|}
\hline Momentum Usage & $15 \mathrm{~Hz}$ & $20 \mathrm{~Hz}$ \\
\hline with $\pm 12 \mathrm{MeV} \&$ "On-Energy" Injection & $0.72 \%$ & $0.86 \%$ \\
with $\pm 12 \mathrm{MeV} \&$ "Off-Energy" Injection & $\mathbf{0 . 5 8} \mathbf{\%}$ & $0.67 \%$ \\
\hline with $\pm 8 \mathrm{MeV} \&$ "On-Energy" Injection & $0.63 \%$ & $0.76 \%$ \\
with $\pm 8 \mathrm{MeV} \&$ "Off-Energy" Injection & $\mathbf{0 . 4 8} \%$ & $\mathbf{0 . 5 7} \%$ \\
\hline with $\pm 4 \mathrm{MeV} \&$ "On-Energy" Injection & $\mathbf{0 . 5 2} \%$ & $0.66 \%$ \\
with $\pm 4 \mathrm{MeV} \&$ "Off-Energy" Injection & $\mathbf{0 . 3 8} \%$ & $\mathbf{0 . 4 7} \%$ \\
\hline
\end{tabular}

Table 3.3: The required momentum aperture for slip-stacking in the Recycler depending on the momentum range of the incoming beam, the injection scheme, and the Booster cyclerate. Fig. 3.12 depicts the two injection schemes. Bolded values pass a $0.6 \%$ benchmark.

A $20-\mathrm{Hz}$ Booster would best be implemented in conjunction with a change in the slipstacking injection scheme to avoid encountering limits in momentum aperture. Kourbanis measured the Recycler momentum aperture in May 2014 to be $0.74 \%$ for $95 \%$ transmission and $0.53 \%$ for $99 \%$ transmission [77]. It should be noted that this momentum aperture is limited by the dynamic aperture, which means that it is sensitive to chromaticity and betatron tuning; it is less than half of the physical aperture, the momentum aperture the could conceivably be achieved with improvements to the lattice (see [78]). The Recycler chromaticity is tuned between -5 and -10 to Landau damp the transverse space-charge beam-coupling impedance instability [42].

The total momentum range used during slip-stacking is shown in Table 3.3. The $20-\mathrm{Hz}$ Booster requires greater RF frequency separation and therefore the total momentum used in any injection scheme would increase. However as Table 3.3 indicates, switching from the "On-Energy" injection with a 15-Hz Booster (status quo) to "Off-Energy" injection 20-Hz Booster (proposed) is actually a net decrease in the total momentum usage. These two injection schemes are depicted in Fig. 3.12.

In the "On-Energy" injection scheme (see Fig. 3.12 left), the extraction energy from the 

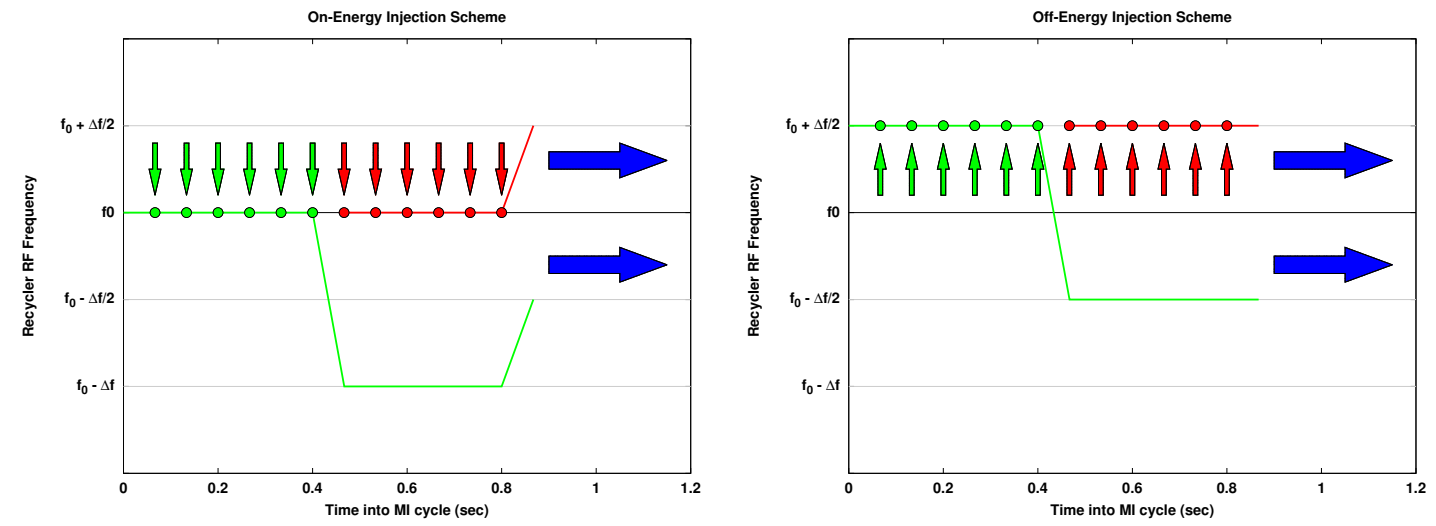

Figure 3.12: (left) The On-Energy injection scheme spans the frequencies $f_{0}-\Delta f$ to $f_{0}+$ $\Delta f / 2$. (right) The Off-Energy injection scheme spans the frequencies $f_{0}-\Delta f / 2$ to $f_{0}+\Delta f / 2$.

Booster is the injection energy into the Main Injector. The frequencies of the Recycler RF cavities move to ensure the injection and extraction is simple, but at the cost of greater momentum usage. In the "Off-Energy" injection scheme (see Fig. 3.12 right), the extraction from the Booster is at a momentum $\Delta \delta / 2$ lower or higher than the momentum of the beam injected into the Main Injector ( [13], p. 8-109). In the "Off-Energy" scheme, the Booster must be tuned separately for injection into the Recycler for slip-stacking and for the 8-GeV beamline. The advantage offered by this alternate injection scheme is that only $\Delta \delta$ and the full bucket height must be accommodated, rather than the $(3 / 2) \Delta \delta$ and the full bucket height required by the On-Energy injection scheme. Eq. 3.1 relates the frequency difference with the momentum difference.

\subsection{Conclusion}

In summary, we have provided a framework for addressing both stability of particles in a slip-stacking potential. We introduce the slip-stacking area factor $F\left(\alpha_{s}\right)$ and the modified area factor $Z\left(\alpha_{s}\right)$ as tools to calculate the stable slip-stacking bucket area for any combination of accelerator parameters. We obtain an explicit general perturbative solution for 
the trajectory of stable slip-stacking particles. We describe a series of new parametric resonances in slip-stacking. We provide a general method for analyzing slip-stacking injection scenarios. We identify how the dynamics of slip-stacking correspond to the driven pendulum and moving standing wave traps.

We demonstrate that the $20-\mathrm{Hz}$ Booster cycle rate provides a consequential improvement to the slip-stacking efficiency and bucket area. We recommend an injection scheme which wholly compensates for the the increased momentum usage required by the larger RF frequency separation. We predict the optimal RV cavity voltage for the $20-\mathrm{Hz}$ Booster and identify the potential overheating issue. 


\section{CHAPTER 4}

\section{Slip-stacking Loss Measurements}

In this chapter, we measure the longitudinal phase-space distribution of the beam immediately before slip-stacking and we also measure the losses in the Main Injector attributed to slip-stacking in the Recycler. Then we simulate the same process and compare it to our results. The reasonable agreement between measurement and simulation validates the numerical approach in the previous (and next) chapter. A critical part of controlling losses in the Main Injector was identifying sources of loss [5] and in this way our study supports the Recycler commissioning effort.

In particular, the experiment described in this chapter allows us to directly measure the impact of the $20 \mathrm{~Hz}$ Booster upgrade on slip-stacking. A Booster cycle-rate of $15 \mathrm{~Hz}$ corresponds to a $1260 \mathrm{~Hz}$ RF frequency separation in the Recycler and when Booster cyclerate of $20 \mathrm{HZ}$ corresponds to a $1680 \mathrm{~Hz}$ RF frequency separation in the Recycler. In this experiment, we vary the RF frequency separation in the Recycler (while the Booster ramp remains the same).

\subsection{Tomography Measurements of Beam Distribution}

Detailed analysis of slip-stacking requires a realistic model of the phase-space distribution of the injected beam. We used the tomography program developed by Evans [79] to obtain a measurement of the longitudinal distribution of the beam injected into the Recycler. 
Fig. 4.1 shows the measurement of the longitudinal distribution of the beam from a typical $2+6$ slip-stacking cycle.
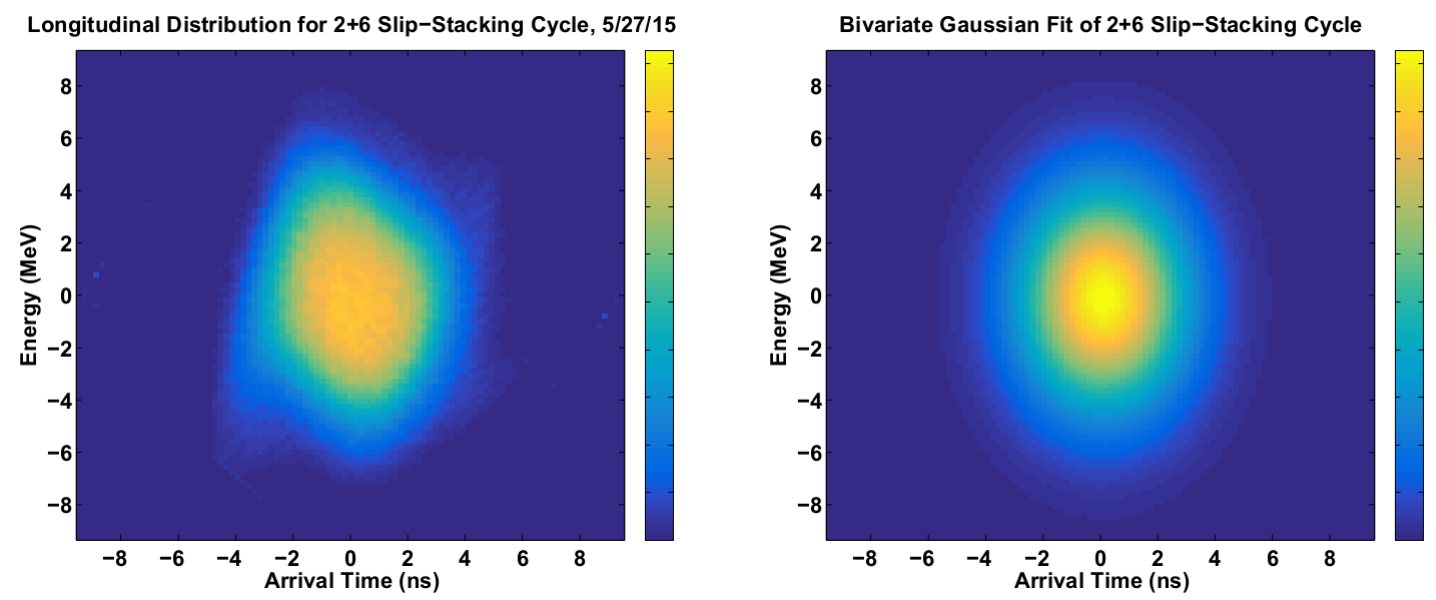

Figure 4.1: (left) Tomography measurements of longitudinal distribution of beam injected into the Recycler. Distribution calculated from an average across Booster batch. Measurement taken from a typical $2+6$ slip-stacking on May 27 th 2015 at a bunch intensity of $5.1 \times 10^{10}$ protons. (right) Bigaussian fit of the measured longitudinal distribution.

The longitudinal distribution obtained from measurement was fit with a bivariate gaussian distribution. The resulting fit is shown in Fig. 4.1 and the resulting fit parameters are given in Table 4.1. A similar measurement made by Seiya et. al. [80] in 2007 found about $70 \%$ greater longitudinal emittance at a comparable beam intensity. The fit, compared to the direct measurement, has smoother and longer tails.

\subsection{Overview of Slip-stacking Beam Loss Study}

The analysis of slip-stacking in this dissertation focuses primarily on losses due to singleparticle longitudinal dynamics. To understand the impact of this work on operationally observed slip-stacking losses, we designed a study to isolate these losses from other losses that may occur in the proton cycle. 


\begin{tabular}{|l|l|}
\hline Temporal Mean $\left(\mu_{t}\right)$ & $+0.11 \mathrm{~ns}$ \\
Energy Mean $\left(\mu_{E}\right)$ & $-0.12 \mathrm{MeV}$ \\
Temporal Sigma $\left(\sigma_{t}\right)$ & $\pm 2.86 \mathrm{~ns}$ \\
Energy Sigma $\left(\sigma_{E}\right)$ & $\pm 4.12 \mathrm{MeV}$ \\
$97 \%$ Emittance $\left(\epsilon_{97 \%}\right)$ & $0.17 \mathrm{eV} \cdot \mathrm{s}$ \\
$99 \%$ Emittance $\left(\epsilon_{99 \%}\right)$ & $0.25 \mathrm{eV} \cdot \mathrm{s}$ \\
Aspect Ratio $\left(\sigma_{p} / \sigma_{t}\right)$ & $1.45 \mathrm{MeV} / \mathrm{ns}$ \\
\hline
\end{tabular}

Table 4.1: Parameters for gaussian fit of the longitudinal distribution measured by tomography.

Fig. 4.2 shows the custom beam cycle used to analyze slip-stacking losses. The beam is injected into the Recycler synchronized with one RF cavity and perturbed by the other RF cavity. The beam loss in the Recycler and the Main Injector is measured in the beam cycle and the cycle is repeated many times with different Recycler RF parameters. The first parameter varied between cycles is the RF voltage of the two Recycler cavities $-25 \mathrm{kV}$, $40 \mathrm{kV}, 55 \mathrm{kV}, 70 \mathrm{kV}$, or $85 \mathrm{kV}$ - which was kept the same for both RF cavities. The second parameter varied is the frequency separation between the two RF cavities $-840 \mathrm{~Hz}, 1050 \mathrm{~Hz}$, $1260 \mathrm{~Hz}$ or $1680 \mathrm{~Hz}$ - with the first RF cavity frequency fixed and the second RF cavity frequency a variable amount lower. A control case in which the the second RF cavity was turned off, was also measured. These parameters change the slip-stacking parameter $\alpha_{s}$ and consequently affect the stable phase-space area of the slip-stacking buckets (see previous chapter).

The single-particle longitudinal dynamics of slip-stacking determines the stable phasespace area of the slip-stacking buckets. Particles not captured in the slip-stacking buckets instead slip with respect to both beams in the Recycler. Those slipping particles may be lost during extraction to the Main Injector, and those that are transferred to the Main Injector are highly unlikely to be captured in the RF bucket of the Main Injector. Particles not 


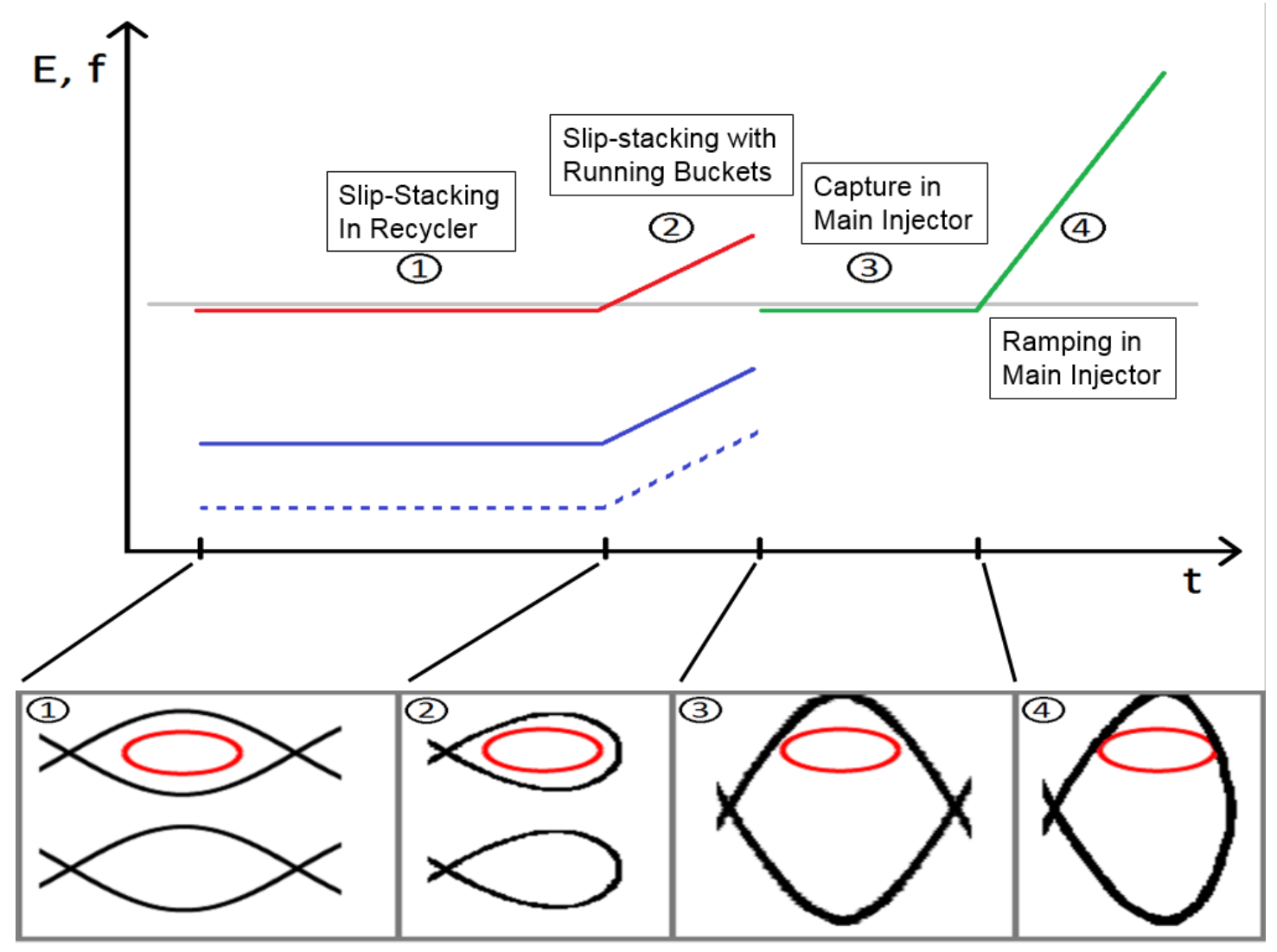

Figure 4.2: (1) A single low-intensity Booster batch is injected into the Recycler, synchronized with one RF cavity and stored for six Booster ticks. The perturbs the stored beam from a fixed frequency separation. (2) The frequency of both RF cavities is ramped linearly in the Recycler during the last Booster tick. The extraction energy of the beam matches the extraction energy of the upper beam in a typical slip-stacking cycle. (3) The Recycler beam is extracted and captured by a large RF bucket in the Main Injector. (4) The beam is accelerated according to the normal MI ramp cycle (see Table 2.3. 
captured in RF bucket of the Main Injector are lost when the beam is accelerated. Particles capture near the MI bucket boundary may be also lost during transition crossing. Fig. 4.3 shows particles losses in the Main Injector associated with slip-stacked beam as measured by $I: B E A M$.

A primary goal of this loss study is to distinguish these losses unique to slip-stacking from operational losses attributed to other effects. The study uses a slip-stacking cycle with a single low intensity batch $\left(1.5 \times 10^{12}\right.$ protons $)$ to reduce losses directly associated with beam-loading or collective effects. The energy of the slip-stacking beam does not vary between cycles and therefore losses associated with insufficient momentum aperture are held constant across all cycles. The transverse optics are also held constant across all cycles.

The slip-stacking loss study took place on June 11th and July 2nd 2015 concurrent with commissioning of the Recycler for $2+6$ slip-stacking [15] and record beam power to the NOvA experiment [16]. The slip-stacking loss study used the E1 event, a study cycle that occurs once every one-minute supercycle and does not interfere with Fermilab neutrino production. Ten cycles were measured for each of the five Recycler RF voltage conditions $(25 \mathrm{kV}, 40 \mathrm{kV}, 55 \mathrm{kV}, 70 \mathrm{kV}, 85 \mathrm{kV})$, for each of five Recycler RF frequency separation conditions $(840 \mathrm{~Hz}, 1050 \mathrm{~Hz}, 1260 \mathrm{~Hz}, 1680 \mathrm{~Hz}$, second RF off), and for each of the two runs (June 11th and July 2nd).

\subsection{Loss Study Results}

The beam losses observed in this study are inferred by decreases in beam intensity measured by Direct Current Beam Current Transformers (DCCTs) [81]. The Fermilab ACNET control system [82] was used to log the beam intensity thorough-out the study. The beam was measured between the Booster and the Recycler using R:TOR853, measured in the Recycler using R:BEAM, measured between the Recycler and the Main Injector using R:T0R905, and 


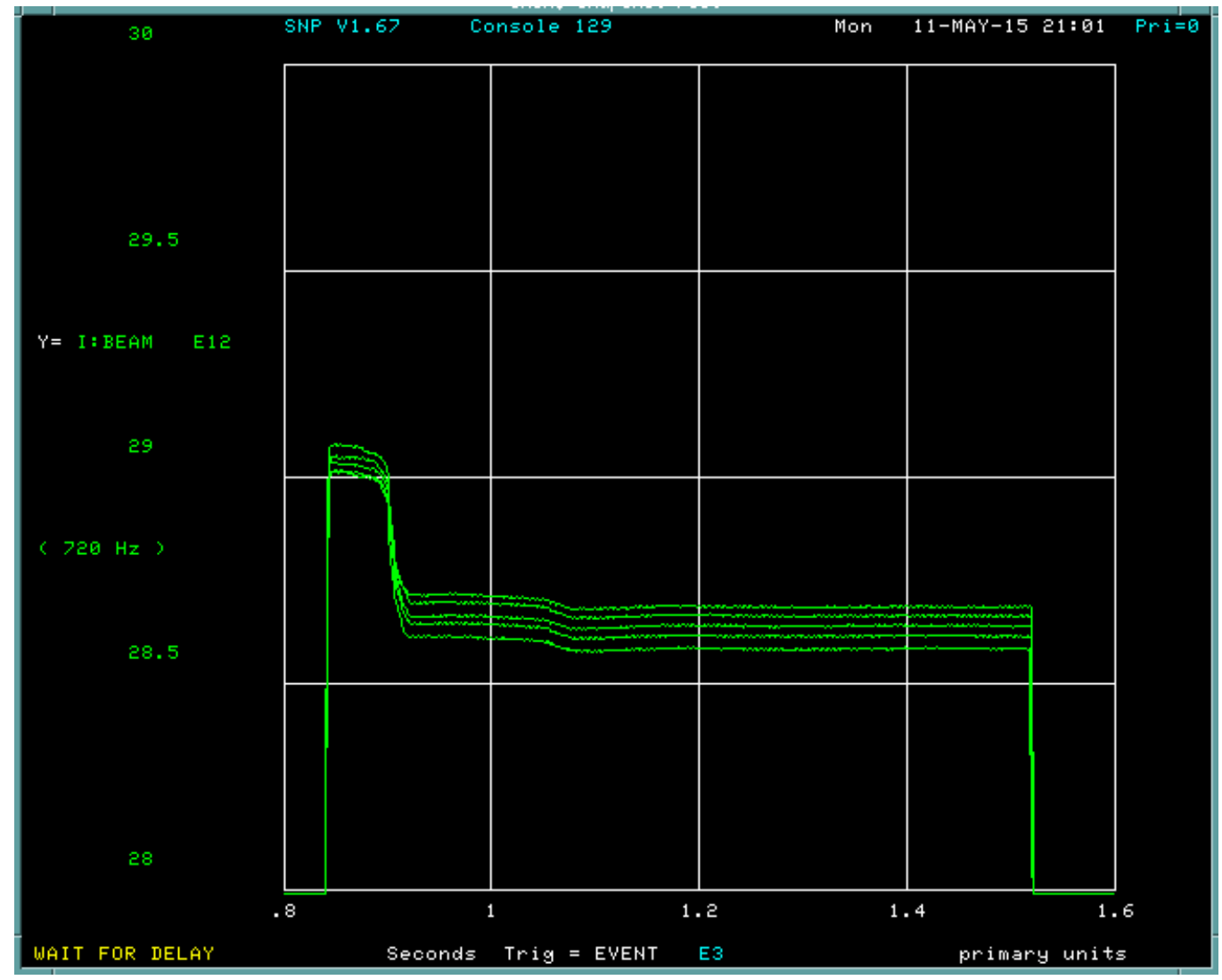

Figure 4.3: Beam intensity in the Main Injector for a typical $2+6$ slip-stacking cycle. The majority of losses occur in the first $100 \mathrm{~ms}$ after injection into the Main Injector. This occurs because the captured beam is accelerated and then remaining particles are left behind and ultimately lost. At the 1.1 second mark, a smaller beam loss is incurred when transition energy is crossed in the Main Injector. 
measured in the Main Injector using I:BEAM. These DCCTs respond linearly with beam intensity but are not calibrated to each other. The DCCTs also have a known dependence on beam quality parameters such as bunch length [81] that may not be the same for all DCCTs. Variation in beam quality prevented us from relating beam intensity in the Recycler to beam intensity in the Main Injector, therefore losses during extraction from the Recycler could not be measured. However beam losses in the Main Injector could be measured (see Fig. 4.3) and these losses are the dominant part of our signal.

Fig. 4.4 shows the fraction of particles lost in the Main Injector for each RF voltage and RF frequency separation case. The qualitative features of these results are consistent with the results of the previous chapter. The greater the frequency separation between the RF cavities, the lower the loss rates are. When the frequency separation is low $(840 \mathrm{~Hz}$ and $1050 \mathrm{~Hz}$ ), the slip-stacking parameter $\alpha_{s}$ is too low and the stable phase-space area is maximized with minimal RF voltage. Conversely, at high frequency separation $(1680 \mathrm{~Hz})$ there is no increase in loss rates with higher $\mathrm{RF}$ voltage. For a moderate frequency separation $(1260 \mathrm{~Hz})$ the optimal RF voltage is some intermediate value.

The apparent overlap of the $840 \mathrm{~Hz}$ and $1050 \mathrm{~Hz}$ loss data is not expected. One possible explanation would be if $840 \mathrm{~Hz}$ lost a greater number of particles during extraction from the Recycler and therefore those losses were not recorded in the Main Injector. Another possible explanation is systematic error between the $840 \mathrm{~Hz}$ and $1050 \mathrm{~Hz}$ datasets which generally took place at least an hour apart and may have differed in beam quality.

The error bars shown in Fig. 4.4 are caused by significant cycle-to-cycle variation as well as significant variation between the two runs. Fig. 4.5 shows the results with each run averaged separately. Fig. 4.6 shows several $70 \mathrm{kV} 1260 \mathrm{~Hz}$ datasets from each run. This variation in loss rate likely represents a variation in Booster beam quality. This represents a systematic error up to $1.5 \%$ an may explain the relationship between the $840 \mathrm{~Hz}$ and $1050 \mathrm{~Hz}$ 
Experimental Slip-stacking Loss Rates

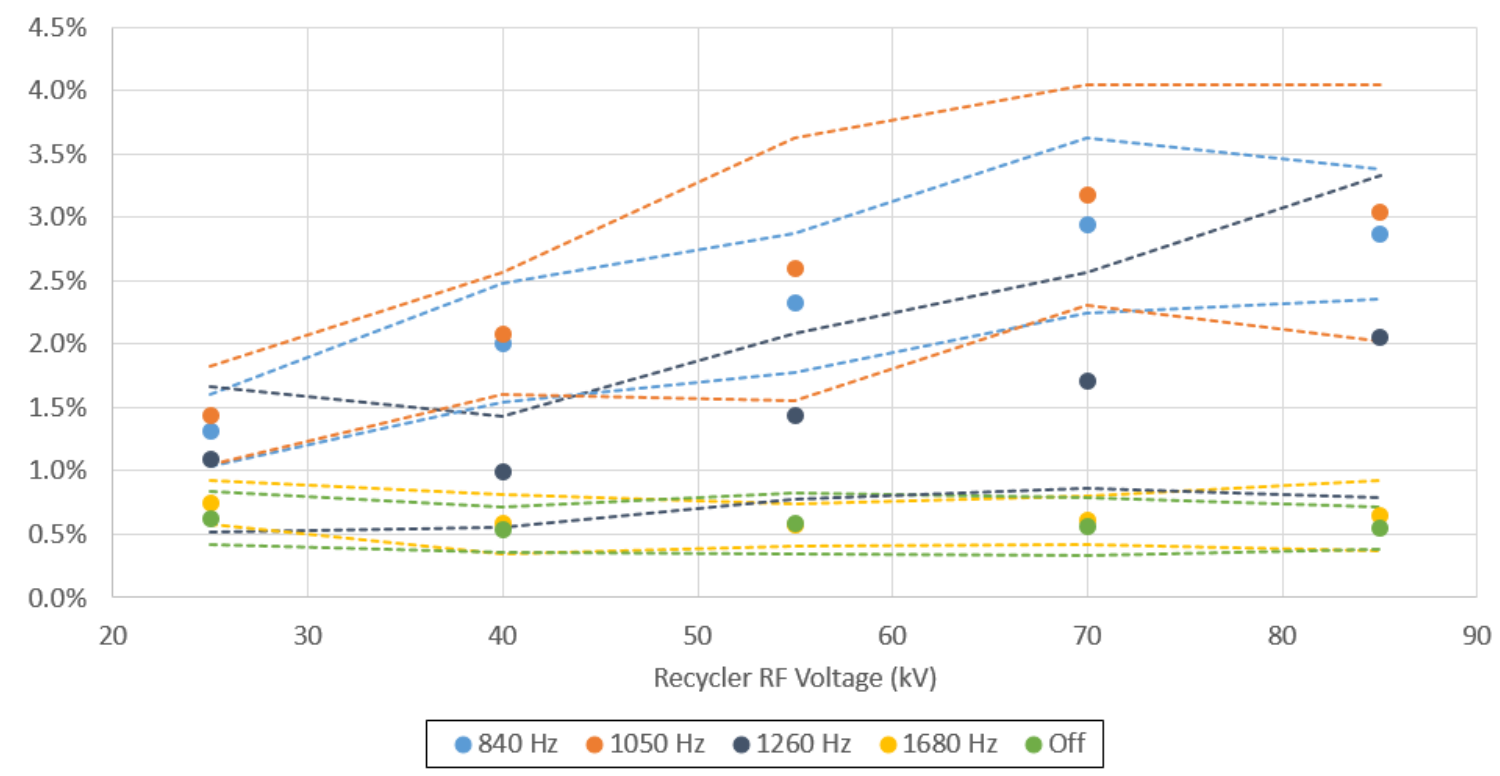

Figure 4.4: The fraction of particles lost in the Main Injector as a function of RF voltage and RF frequency separation in the Recycler. The points indicate the average beam loss across all cycles with the same Recycler RF parameters (in both runs) and the corresponding dashed lines indicate a one standard deviation margin around this average. 
datasets. Although the beam intensity drifts within a range of $\pm 10 \%$ over several hours, the variation in beam quality did not depend on beam intensity (as it did in [80]). The variation in the beam quality can be caused by changing conditions in the Booster, Linac, or $\mathrm{H}^{-}$Source.

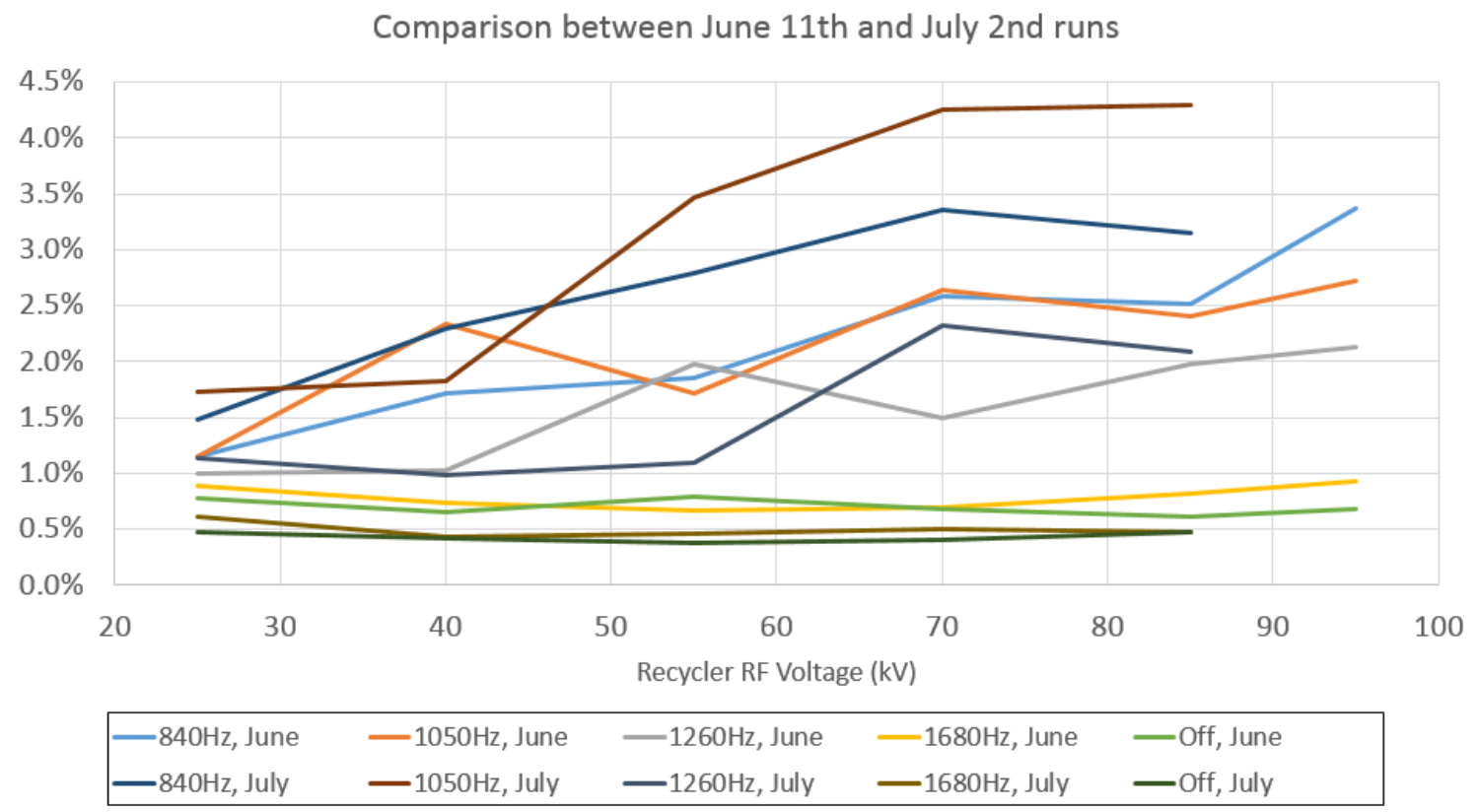

Figure 4.5: The fraction of particles lost in the Main Injector as a function of RF voltage and RF frequency separation in the Recycler. The lines indicate the average beam loss across all cycles with the same Recycler RF parameters for a particular run (June 11th or July 2nd). The discrepancy between the averages obtained for each of the two runs can be as large as $1.5 \%$.

The datasets with $1680 \mathrm{~Hz}$ RF frequency separation and with no second RF off coincide almost completely. This indicates that the slip-stacking perturbation is negligible at this frequency separation (for RF voltages less then $100 \mathrm{kV}$ and with the current beam emittance). Recall that slip-stacking as it currently operates in the Recycler has a $1260 \mathrm{~Hz} \mathrm{RF}$ frequency separation that the $1680 \mathrm{~Hz}$ RF frequency separation would be operational if the Booster cycle-rate is upgraded to $20 \mathrm{~Hz}$ as part of PIP-II [63]. This study indicates that 


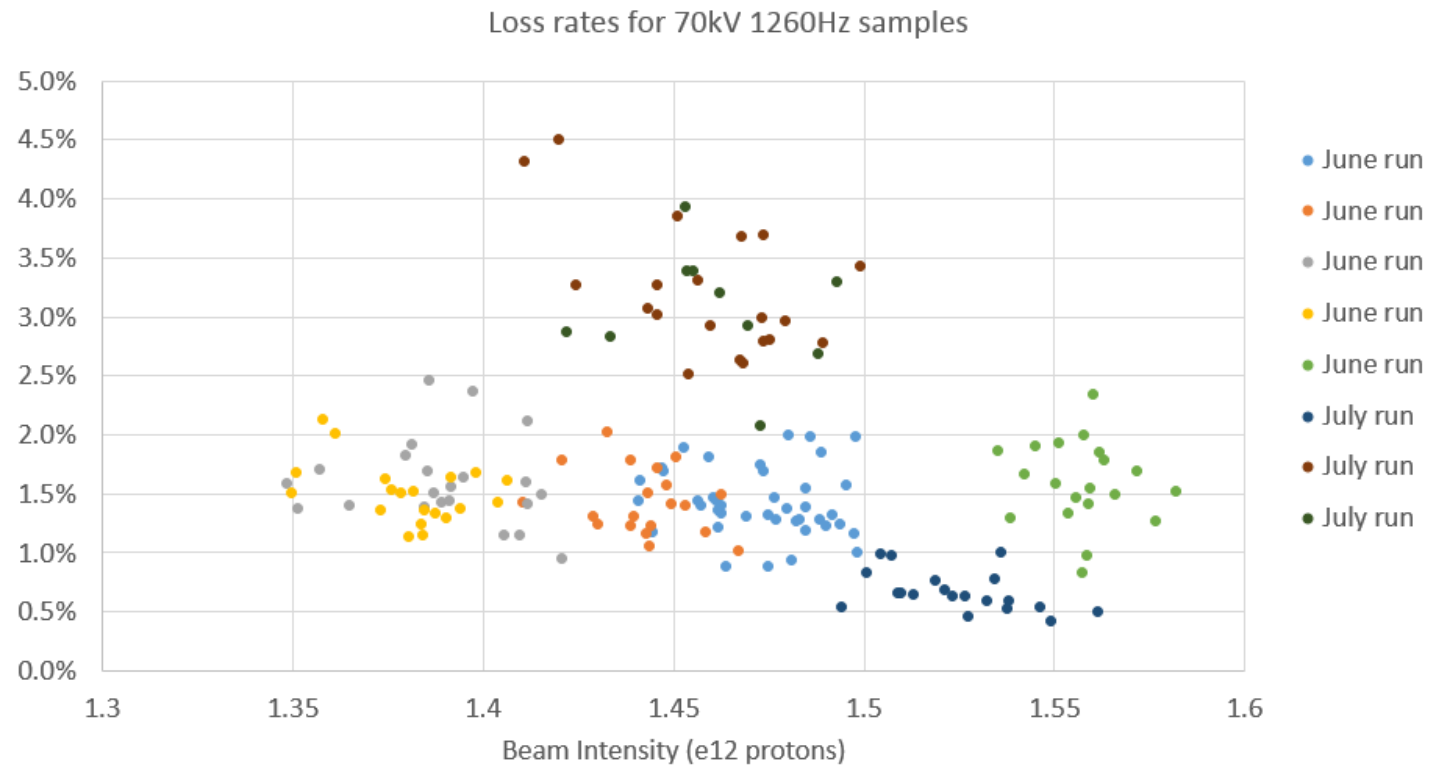

Figure 4.6: The $70 \mathrm{kV} 1260 \mathrm{~Hz}$ dataset was measured several times during each run. Each cycle is plotted as a function of beam intensity and loss rate. The samples taken during the June 11th run and the July 2nd run are light and dark respectively. The variation in beam quality is significant. 
losses in the Main Injector will be reduced by at least a factor of 2 (from $1 \%$ to $0.5 \%$ ).

There is an apparent minimum $0.5 \% \pm 0.2 \%$ particle loss that persists even with the Recycler RF voltage maximized and with the second RF cavity off (see Fig. 4.4 and Fig. 4.5). Therefore this loss cannot be caused by a slip-stacking perturbation from the second RF cavity. However, these particles are lost after the start of the Main Injector ramp, like other slip-stacking loss (see Fig. 4.3). This constant source of loss is unexpected and several hypotheses were considered. If these losses corresponded to off-momentum particles not captured by the Recycler RF, one would expect the losses to be sensitive to Recycler RF voltage. The momentum acceptance of the Main Injector RF bucket is higher then that of the Recycler RF bucket, so these particle are not lost due to inadequate RF voltage in the Main Injector either. Studies with lower intensity batches $\left(0.5 \times 10^{12}\right.$ protons $)$ indicate that beam-loading and collective effects account for no more then $0.15 \%$ particle loss. Losses from transition crossing in the Main Injector were measured to be less than $0.05 \%$ particle loss. We believe that the losses corresponds to particles off-phase and off-momentum not captured by the Recycler RF for any voltage, as depicted in Fig. 4.7. Nonlinearities in the bunch rotation process [35] could regularly create this distorted distribution of particles, as shown in Fig. 2.9. Such particles would not be apparent in the tomography measurements which are only designed to measure captured particles.

\subsection{Simulation of Loss Study}

In this section, we report on our simulation of the slip-stacking beam loss study described in the preceding sections. The initial longitudinal distribution used for the simulation analysis was taken from the tomography measurements shown in Fig. 4.1. For comparison, the fit of the tomography measurements (Fig. 4.1 and Table 4.1) were also used in an independent and identical simulation. The initial longitudinal distribution was numerically integrated 


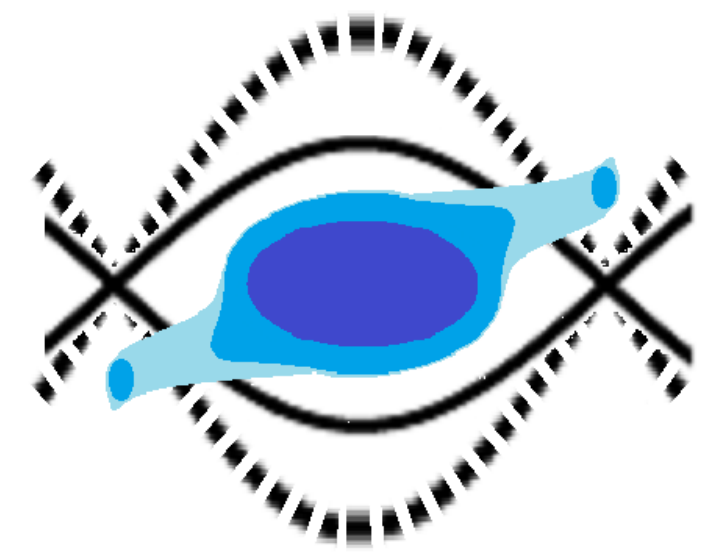

Figure 4.7: The blue contours indicate a hypothetical longitudinal beam distribution extracted from the Booster to the Recycler ( $\phi$ horizontal, $\delta$ vertical). The black lines a simple RF bucket depicted at two RF voltages in the Recycler. In this scenario, the tails of this beam distribution are not be sensitive to changes in RF voltage.

through the four stages depicted in Fig. 4.2, including the details of the Main Injector ramp given in Table 2.3. Particles were considered lost when they fell outside the momentum aperture of the Main Injector (when they failed to be captured in the Main Injector RF bucket). The simulation was repeated for each of five Recycler RF frequency separation conditions $(840 \mathrm{~Hz}, 1050 \mathrm{~Hz}, 1260 \mathrm{~Hz}, 1680 \mathrm{~Hz}$, second RF off) and at Recycler RF voltages from $20 \mathrm{kV}$ to $100 \mathrm{kV}$ in steps of $5 \mathrm{kV}$.

Fig. 4.8 shows the simulated losses for the tomography measurement input and the bigaussian fit input. The experimentally measured losses that are shown in Fig. 4.4 are also reproduced in Fig. 4.8, except that the $0.5 \%$ constant loss has been subtracted off. Overall, the simulated losses show the same qualitative behavior as the measured loss and roughly the same magnitude. The simulation with the measurement input falls close to the error bars for experimental data for the $840 \mathrm{~Hz}, 1680 \mathrm{~Hz}$, and Off datasets.

In the $1050 \mathrm{~Hz}$ dataset and the $1260 \mathrm{~Hz}$ dataset, the discrepancy between simulation 
and data is more pronounced. In the $1050 \mathrm{~Hz}$ dataset the experimental losses appear to be too high and the RF voltage with minimal losses is predicted to be closer to $40 \mathrm{kV}$ than $25 \mathrm{kV}$. Systematic error caused by the variation in beam quality is the best explanation. In the $1260 \mathrm{~Hz}$ dataset the $\mathrm{RF}$ voltage with minimal losses is predicted to be close to $70 \mathrm{kV}$ but measured to be closer to $40 \mathrm{kV}$. This is consistent with the simulation having a higher aspect ratio (momentum spread to temporal spread ratio) than the average beam in the experimental study. There are three possibilities to consider. The first possibility is that the tomography measurement is unrepresentative of the typical beam during the study. The second possibility is that an unaccounted for excitation of the beam (e.g. beam-loading)

increases the temporal spread of the beam and contributes to slip-stacking losses. The third possibility is that particles loss during extraction from the Recycler is significant and disproportionately off-momentum.

\subsection{Conclusion}

A tomography measurement was made of the longitudinal phase-space and we demonstrated this data can be applied to slip-stacking simulation and optimization. A custom beam cycle was designed to isolated losses associated with the single-particle longitudinal dynamics of slip-stacking. Within the limitations of beam quality, the loss data as a function of RF voltage and RF frequency separation fit those expectations. At a given RF frequency separation, the optimal RF voltage was observed to be a trade-off between increasing bucket height and increasing slip-stacking perturbation. As the RF frequency separation increased, the losses were observed to decrease at a given RF voltage.

The custom beam cycle was simulated and the simulated losses were consistent with the measured losses, up to limitations in beam quality. This validates the simulation approach taken in the previous and next chapter, which exclude collective effects. 

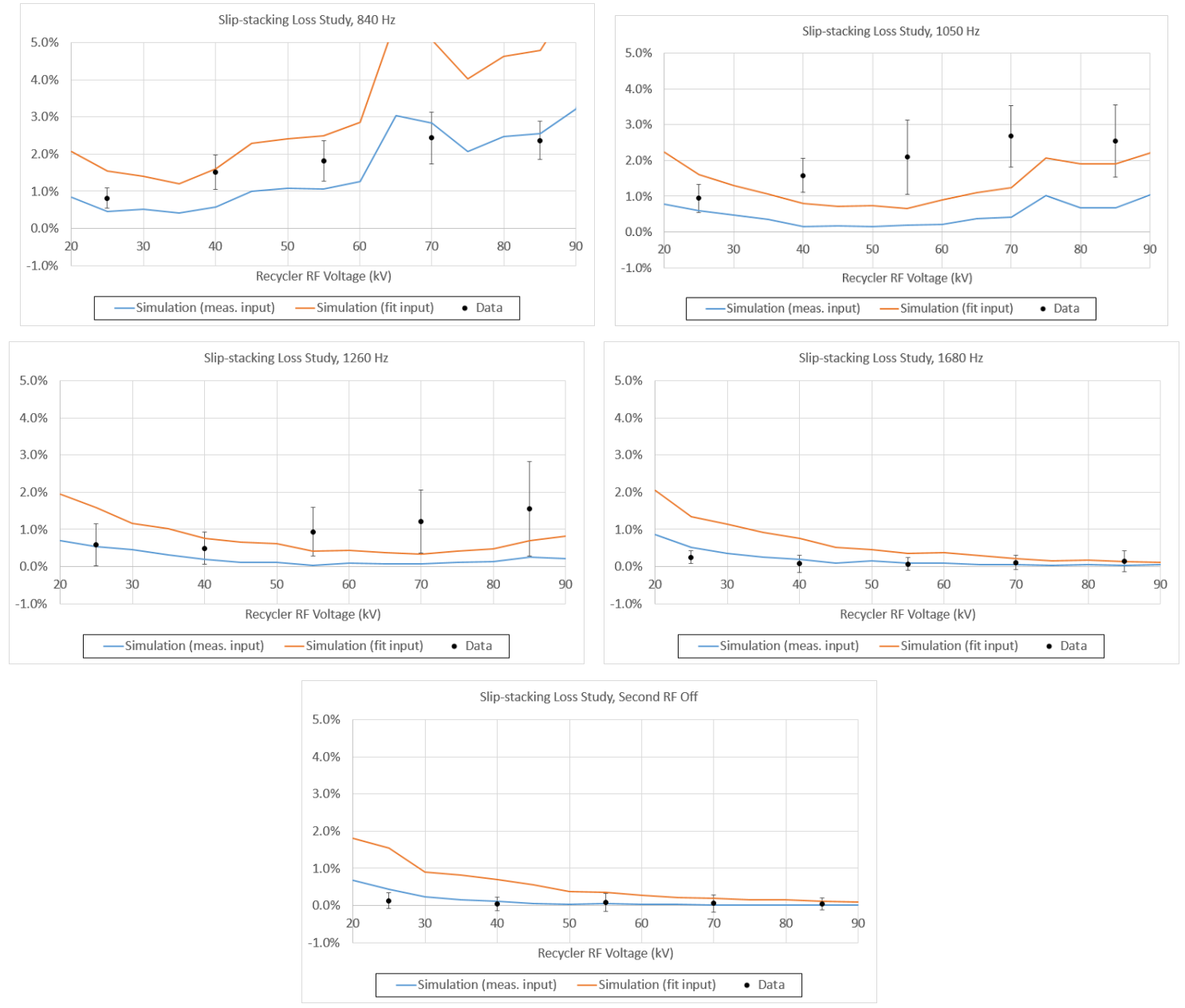

Figure 4.8: The fraction of particles lost in the Main Injector as a function of Recycler RF voltage, for each RF frequency separation condition $(840 \mathrm{~Hz}, 1050 \mathrm{~Hz}, 1260 \mathrm{~Hz}, 1680 \mathrm{~Hz}, 2 \mathrm{nd}$ RF off). The average experimentally measured losses are black dots with one sigma error bars. The simulated loss are shown in blue (measurement input) and orange (bigaussian fit input). The $1050 \mathrm{~Hz}$ dataset and the $1260 \mathrm{~Hz}$ dataset show the largest discrepancy between experiment and simulation. 
Our results indicate that the upgrade to the $20-\mathrm{Hz}$ Booster cycle-rate as part of PIP-II is expected to reduce slip-stacking losses in the Main Injector by at least a factor of 2. Our study also uncovered a previously unknown source of $0.5 \% \pm 0.2 \%$ beam loss, believed to be caused by Booster bunch rotation. The Booster bunch rotation can be re-tuned for a larger slip-stacking bucket and consequently it could be also possible to reduce these losses. 


\section{CHAPTER 5}

\section{Harmonic Slip-Stacking Dynamics}

In this chapter, we propose the installation of a new $106 \mathrm{MHz} 20 \mathrm{kV}$ harmonic RF cavity to the Fermilab Recycler that will further reduce particle loss during slip-stacking. The tools for analyzing slip-stacking introduced in chapter 3 have been extended in this chapter. In addition, we simulated the emittance and momentum spread of the slip-stacking beam compared to the injected beam.

\subsection{Motivation}

RF cavities operating at a multiple of a main RF cavity are extensively used in high-energy particle accelerators to achieve a variety of dynamical effects. The application of harmonic $\mathrm{RF}$ cavity we propose in this section is novel because in that it does not operate at precise multiple of any one RF cavity, but rather it operates at twice the average of the upper and lower frequency main RF cavities. The harmonic RF cavity is not synchronized to either beam, but helps keep both beams synchronized with their corresponding main RF cavity. We refer to this modification of slip-stacking as "harmonic slip-stacking".

The PIP-II upgrade will increase proton beam power by $70 \%$ from $700 \mathrm{~kW}$ to $1.2 \mathrm{MW}$ and requires at least a $70 \%$ decrease in the slip-stacking loss rate [7]. In chapter 3, we demonstrated that upgrading the Booster cycle-rate to $20 \mathrm{~Hz}$ would substantially reduce beam losses. In this chapter, we propose installing a $106 \mathrm{MHz} 20 \mathrm{kV}$ RF cavity as a proactive 
way to reduce slip-stacking losses ahead of the PIP-II upgrade. The upgrade to the $20 \mathrm{~Hz}$ Booster would then become optional and the primary consideration would be the benefit of the additional power to the $8-\mathrm{GeV}$ beamline [8-10]. Harmonic slip-stacking, especially in conjunction with a $20 \mathrm{~Hz}$ Booster cycle-rate or a Recycler RF cavity voltage upgrade, would prepare this critical accumulation process for substantially higher beam powers.

A PIP-III RCS upgrade would increase the $8-\mathrm{GeV}$ bunch intensity by a factor $2-4$ over PIP-II with the goal achieving a multi-MW proton beam power at $120-\mathrm{GeV}$ [50]. The current slip-stacking loss rates prohibit a significantly higher beam power, but the harmonic RF cavity proposed in this chapter eliminates that obstacle. If the higher intensity beam from a PIP-III RCS can be slip-stacked in the Recycler, the beam power achievable in that scenario is doubled. Alternatively, slip-stacking could reduce the Linac upgrade required to achieve a particular $120 \mathrm{GeV}$ beam power from a hybrid RCS. If slip-stacking cannot occur in the Recycler during PIP-III, slip-stacking in the Main Injector would provide a fractional increase in beam power (depending on the RCS cycle-rate).

CERN is also planning on implementing slip-stacking for ion beams in the Super Proton Synchrotron (SPS) as part of the Large Hadron Collider (LHC) Injector Upgrade (LIU) [83, 84]. Although there is currently no $400 \mathrm{MHz}$ RF cavity in the SPS $[85,86]$, the results of this chapter imply that such a cavity would improve the efficiency or lower the final emittance of the slip-stacking process in the SPS.

\subsection{Slip-stacking with Harmonic RF}

Recall that in regular slip-stacking in the Recycler, the two beams are maintained by two $53 \mathrm{Mhz}$ RF cavities, the upper RF frequency at $f_{0}+\Delta f / 2$ and the lower RF frequency at $f_{0}-\Delta f / 2$. For harmonic slip-stacking in the Recycler, a $106 \mathrm{MHz}$ RF cavity would operate at twice the average frequency $2 f_{0}$. We add a new term to Eq. 3.2 corresponding to this 
new RF cavity:

$$
\begin{aligned}
& \dot{\phi}=2 \pi f_{\text {rev }} h \eta \delta \\
& \dot{\delta}=f_{r e v} V_{\delta}\left[\sin (\phi)+\sin \left(\phi-\omega_{\phi} t\right)+\lambda \sin \left(2 \phi-\omega_{\phi} t\right)\right] .
\end{aligned}
$$

where $\lambda$ is the ratio between the harmonic RF voltage and main RF voltage $\lambda=V_{H} / V_{M}$. Negative values of $\lambda$ correspond to bunch-lengthening mode and positive values of $\lambda$ correspond to bunch-shortening mode. Eq. 1.10 is equivalent to the simple pendulum and Eq. 3.2 is equivalent to the driven pendulum, but there is no clear pendulum analogue for Eq. 5.1.

We write the second-order equation of motion corresponding to Eq. 5.1 and use a Taylor series to expand $\sin \phi$ and $\cos \phi$ as polynomials:

$$
\begin{aligned}
\ddot{\phi}=-\omega_{s}^{2}\left[\sum_{k=0}^{\infty} \frac{(-1)^{k}}{(2 k+1) !} \phi^{2 k+1}\right. & +\sum_{k=0}^{\infty} \frac{(-1)^{k}}{(2 k+1) !} \phi^{2 k+1}\left(1+\lambda 2^{2 k+1}\right) \cos \left(\omega_{\phi} t\right) \\
& \left.-\sum_{k=0}^{\infty} \frac{(-1)^{k}}{(2 k) !} \phi^{2 k}\left(1+\lambda 2^{2 k}\right) \sin \left(\omega_{\phi} t\right)\right] .
\end{aligned}
$$

To understand the role of $\lambda$ in Eq. 5.2, we consider the value of $\phi^{m}\left[1+\lambda 2^{m}\right]$ in the case where $\lambda=-2^{-p}$. For $p=m$ the coefficient is completely canceled, for $p>m$ the bracketed term is positive and less than 1 , and for $p<m$ the bracketed term is negative and with magnitude greater than 1 . The $\phi^{m}$ term generates the lowest order contribution to the $m \omega_{s}(1+\sigma)=\omega_{s}$ resonance.

This suggests a natural scaling of $\lambda$ with $\alpha_{s}$. Suppose that for some value of $\alpha_{s}$ and there is some optimal value of $\lambda$ for which $\lambda=-2^{-m}$ corresponding to some appropriate resonance $m=\omega_{\phi}(1+\sigma) / \omega_{s}=(1+\sigma) \alpha_{s}$. Under the transformation $\alpha_{s} \rightarrow \kappa \alpha_{s}$ we have $m \rightarrow \kappa m$ and $\lambda \rightarrow-2^{-\kappa m}$. Consequently an optimal value of $\lambda$ should have an exponential functional dependence on $\alpha_{s}$ given by

$$
\lambda=-e^{\xi \alpha_{s}}
$$

for some unknown exponential constant $\xi$ (and an exponential coefficient of -1 ). We expect this functional dependence to be valid at high values of $\alpha_{s}$ where resonances are closer and 
approximate the continuous case. In the next section, we show that the value of $\lambda$ which maximizes the phase-space area follows the form given in Eq. 5.3.

\subsection{Stability Maps and Area Factors}

We create a stability map (see chapter 3 ) for each value of the slip-stacking parameter $\alpha_{s}$ and the harmonic-main voltage ratio $\lambda$. We map the stability of initial particle positions by integrating the equations of motion for each initial position. Each position is mapped independently and only the single particle dynamics are considered. A particle is considered lost if its phase with respect to each of the first RF cavity, the second RF cavity, and the average of the two RF cavities, is larger than a certain cut-off (we used $3 \pi / 2$ ). Figure 5.1 shows an example of a stability without a harmonic RF cavity and with a harmonic RF

cavity. Appendix D shows a selection of harmonic slip-stacking stability maps with other values of $\alpha_{s}$ and $\lambda$.

The bucket area is computed as the product of the total number of ultimately surviving points and the cell area. Recall, the slip-stacking area factor $F\left(\alpha_{s}, \lambda\right)$ is the defined to be the ratio between the slip-stacking bucket area to that of a single-RF bucket with the same RF voltage and frequency. The slip-stacking area factor $F\left(\alpha_{s}, \lambda\right)$ provides a method for calculating the slip-stacking stable phase-space area without requiring each case to be simulated individually. Figure 5.2 shows the slip-stacking area factor $F$ as a function of $\alpha_{s}$ and $\lambda$, with each datapoint calculated from its own stability map.

Recall, the modified slip-stacking area factor $Z\left(\alpha_{s}, \lambda\right)$ is defined to be $F\left(\alpha_{s}, \lambda\right) / \alpha_{s}$ and is proportional to the slip-stacking phase-space area with a coefficient independent of voltage. Figure 5.3 shows the modified slip-stacking area factor $Z$ as a function of $\alpha_{s}$ and $\lambda$.

From Figure 5.2 and Figure 5.3 it is clear that for any value of $\alpha_{s}$, there is an optimal value of $\lambda$ which maximizes the phase-space area. We term this the "balanced" condition 


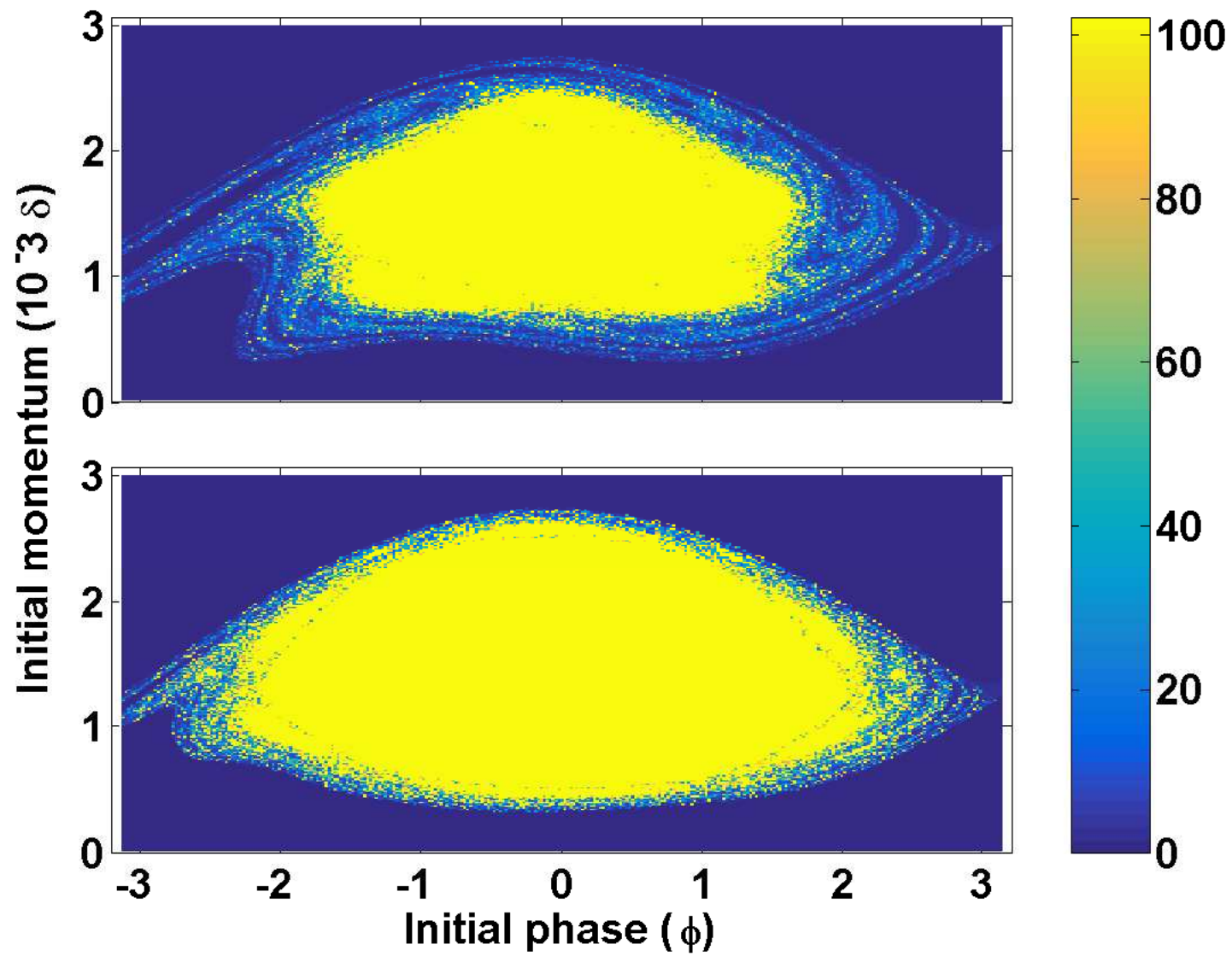

Figure 5.1: Stability of initial coordinates for $\alpha_{s}=4.18$. The color shows the number of synchrotron periods a test particle survives before it is lost. The top plot shows slip-stacking in the status quo. The bottom plot shows slip-stacking enhanced by a harmonic RF cavity. 


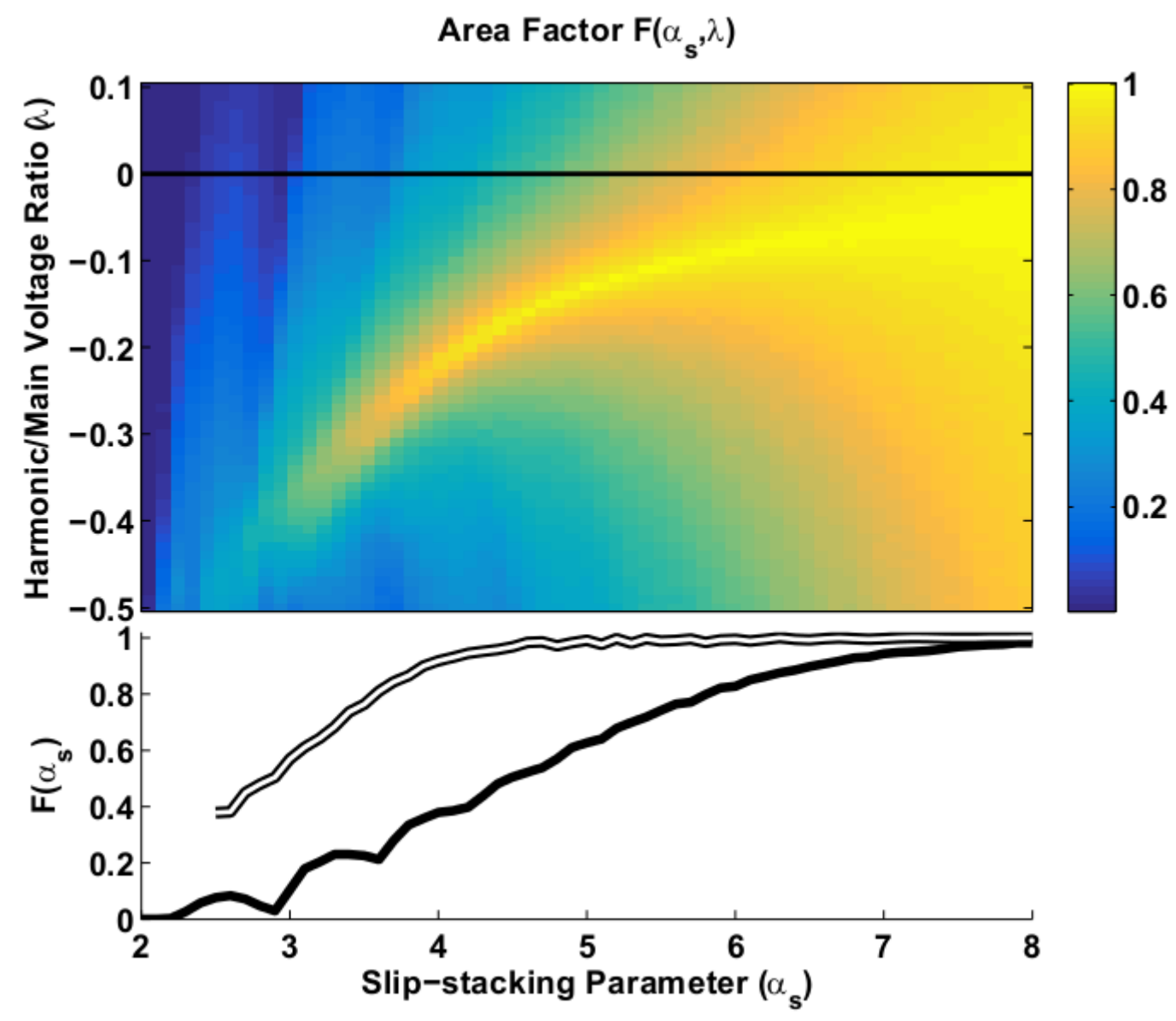

Figure 5.2: (top) Slip-stacking area factor $F$ as a function of $\alpha_{s}$ and $\lambda$. (bottom) Slipstacking area factor $F$ for case without a harmonic cavity $(\lambda=0)$ shown as a single line and case with a harmonic cavity (optimal $\lambda$ ) shown as a double line. 


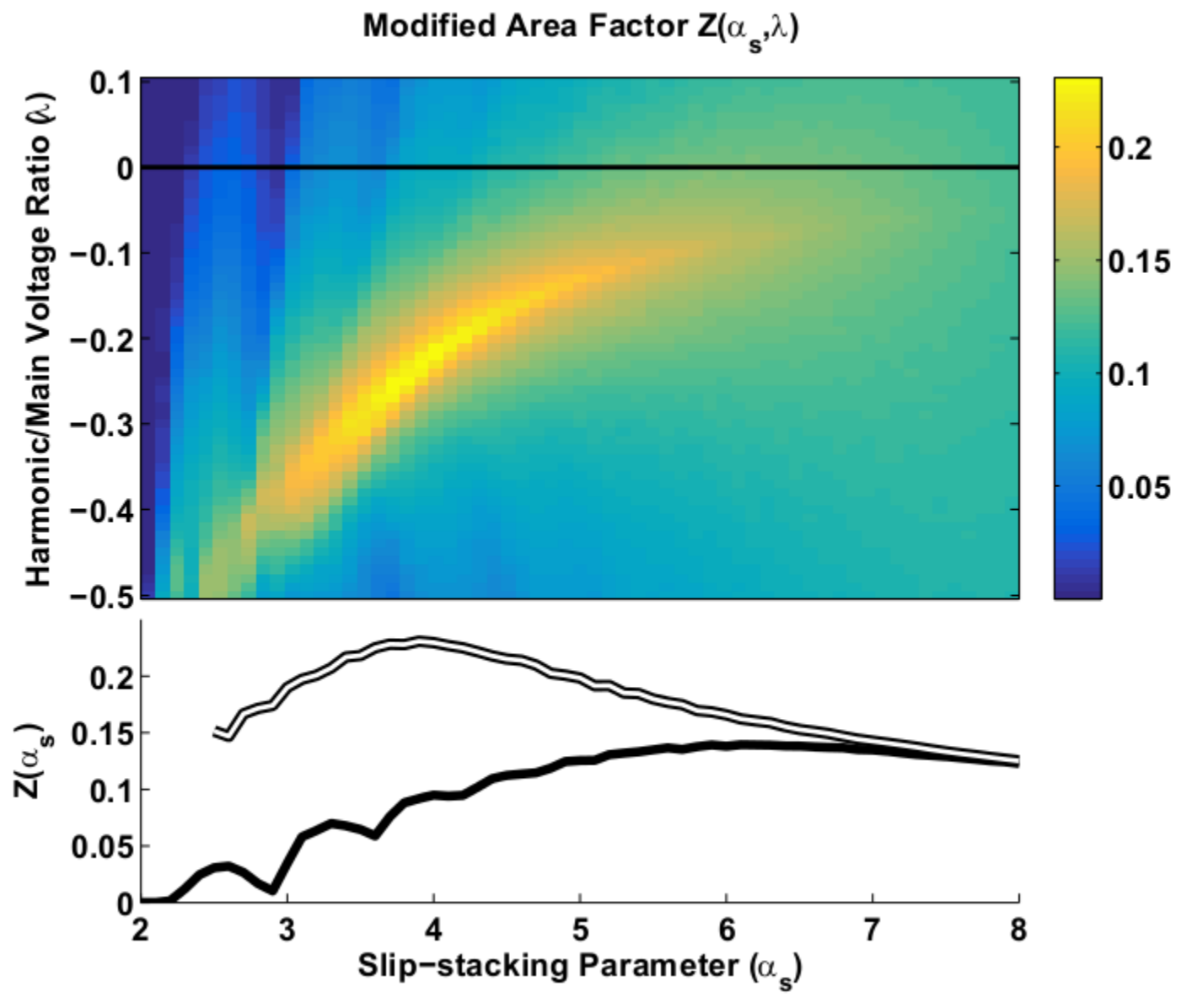

Figure 5.3: (top) Modified slip-stacking area factor $Z$ as a function of $\alpha_{s}$ and $\lambda$. (bottom) Modified slip-stacking area factor $Z$ for case without a harmonic cavity $(\lambda=0)$ shown as a single line and case with a harmonic cavity (optimal $\lambda$ ) shown as a double line. 
for $\lambda$. Figure 5.2 indicates that for $\alpha_{s}>4$ at least $90 \%$ of the stable phase-space area can be recovered by using the balanced condition. Figure 5.3 indicates that the maximum stable phase-space area with harmonic RF is $65 \%$ higher than that without harmonic RF. $Z\left(\alpha_{s}\right)$ is maximized at $\alpha_{S}=3.9$ with harmonic $\mathrm{RF}$ and is maximized at $\alpha_{s}=6.2$ without harmonic RF.

For each value of $\alpha_{s}$, the value of $\lambda$ which maximizes phase-space area is plotted in Fig. 5.4. In Fig. 5.4, the optimal value of $\lambda$ is fit for $\alpha_{s}>4$ with Eq. 5.3:

$$
\lambda \approx-e^{-0.4 \alpha_{s}}, \alpha_{s}>4
$$

This fit is consistent with the resonance-canceling mechanism described in the previous section. Fig. 5.4 also shows an empirically-driven engineering equation given by:

$$
\lambda \approx-\frac{7}{2} \alpha_{s}^{-2}, \alpha_{s}>3
$$

\section{Fits for Balanced $\lambda$}

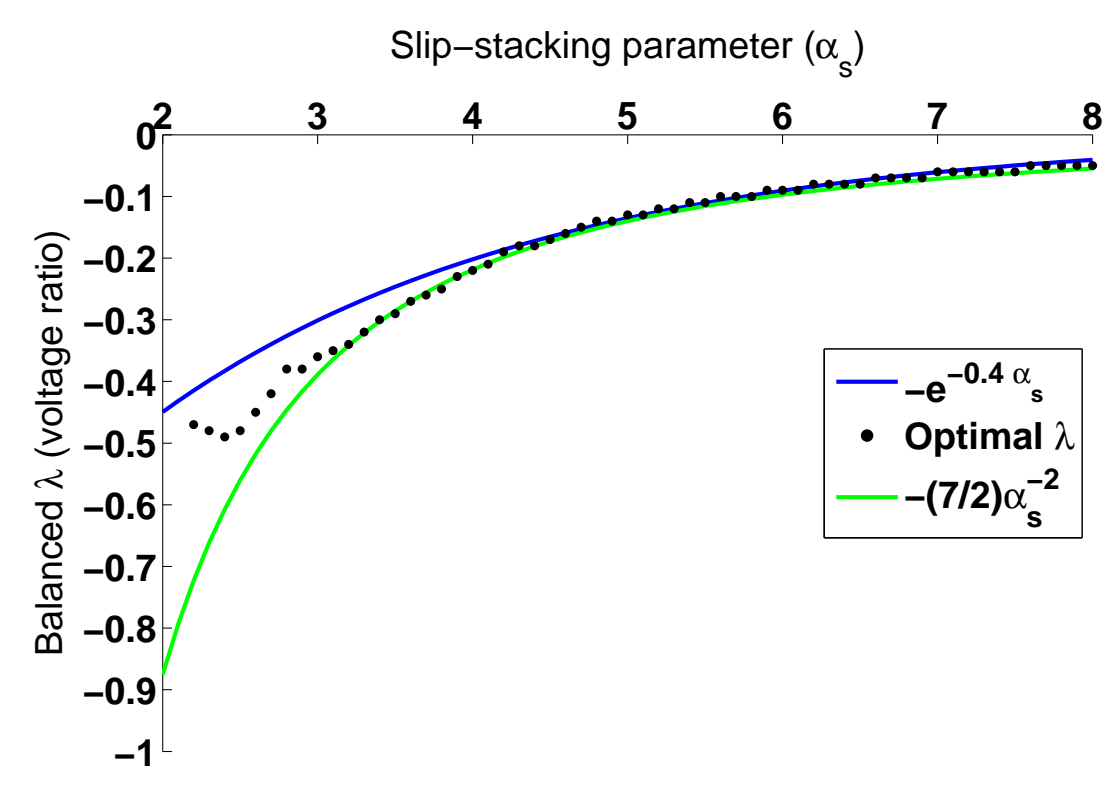

Figure 5.4: Value of $\lambda$ which maximizes phase-space area, as a function of $\alpha_{s}$.

Recall that $\lambda=V_{H} / V_{M}$ and $\alpha_{s}=\alpha_{s}^{(0)} \sqrt{V_{M} / V_{M}^{(0)}}$. If $\lambda$ follows Eq. 5.5 then the harmonic $\mathrm{RF}$ voltage is proportional to the square of the main RF voltage. For Recycler parameters 
(see Table 3.1) and a $15-\mathrm{Hz}$ Booster cycle-rate, we have $V_{H} \approx-1.8 V_{M}^{2}$ where $V_{H}$ and $V_{M}$ are in units of MV. For a $20-\mathrm{Hz}$ Booster cycle rate, we have $V_{H} \approx-1.0 V_{M}^{2}$. Figure 5.5 shows the Harmonic RF voltage that maximizes the phase-space area at each main RF voltage.

Balanced Harmonic Voltage as a function of Main Voltage Main RF Cavity Voltage (kV)

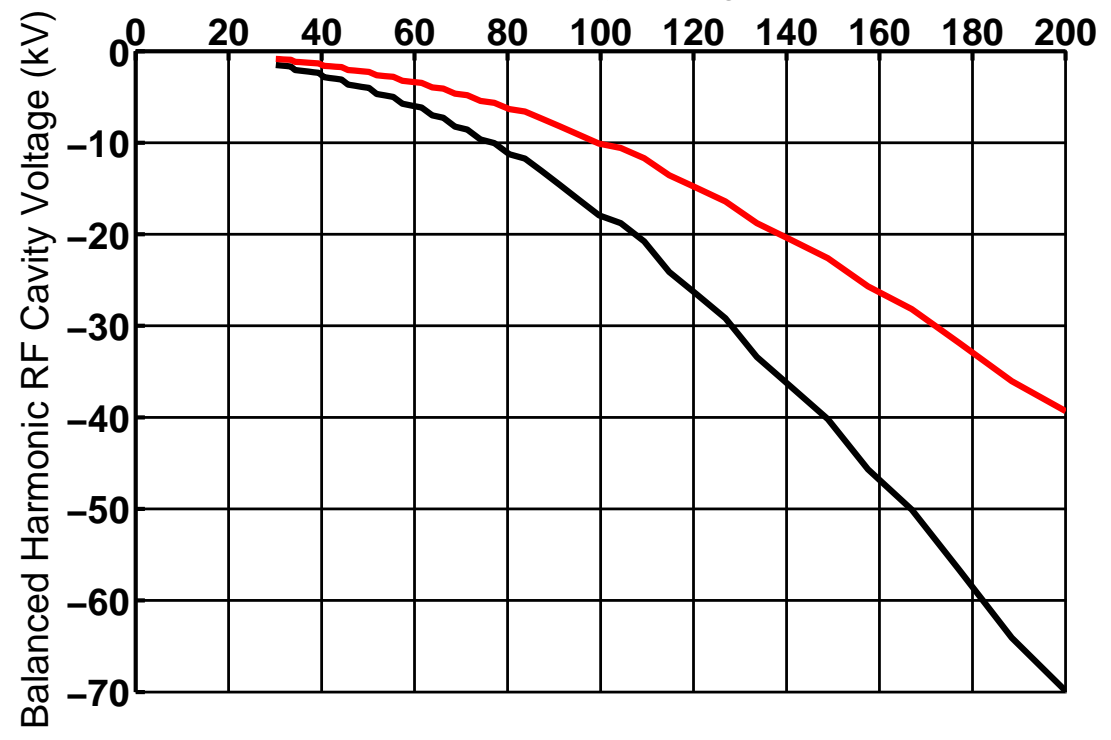

Figure 5.5: Balanced value of harmonic RF voltage has a quadratic dependence on main $\mathrm{RF}$ voltage. Bottom line shows case for a $15-\mathrm{Hz}$ Booster (black) and top line for $20-\mathrm{Hz}$ Booster (red).

\subsection{Poincaré Maps}

Poincaré maps are a tool traditionally used to analyze continuous nonautonomous dynamical systems by clearly indicating fixed points, parametric resonances and regions of chaos (see $[87,88])$. The Poincaré maps presented in this section are obtained by plotting the $\phi-\delta$ phase-space coordinates only at every phase-slipping period $\left(T_{\phi}=2 \pi / \omega_{\phi}\right)$ in a numerical integration of the particle trajectory. We start from 7500 trajectories with initial coordinates uniformly distributed in phase-space and eliminate trajectories corresponding 
to lost particles $(|\phi|>3 \pi / 2)$. The Poincaré maps are drawn using a subset of the remaining trajectories corresponding to approximately even spacing.

Fig. 5.6 shows four Poincaré maps selected to represent the slip-stacking parameter space. Subfigures (a) and (d) have comparable phase-space area. In subfigure (d) the significant negative value of $\lambda$ has changed the orientation of the fourth-order and fifthorder resonances, as expected from Eq. 5.2. Subfigures (b) and (c) have comparable area factors $F\left(\alpha_{s}, \lambda\right)$ and both indicate large regions of smooth phase-space trajectories. These plots indicate that the harmonic slip-stacking with a balanced value of $\lambda$ reduces impact of the slip-stacking perturbation with a success similar to that of increasing the slip-stacking parameter $\alpha_{s}$.

Appendix E contains a selection of harmonic slip-stacking Poincaré maps with other values of $\alpha_{s}$ and $\lambda$.

\subsection{Injection Efficiency of Gaussian Beams}

The stability maps can also be used to analyze injection scenarios, by weighting the (scaled) stability maps according to a distribution that represents the number of incoming particles injected into that region of phase-space. We used this technique to identify $99 \%$ longitudinal admittance, the greatest longitudinal emittance an incoming Gaussian-distributed beam could have and still achieve $99 \%$ injection efficiency (at its optimal value of $\alpha_{s}$ and $\lambda$ ). The $99 \%$ longitudinal beam emittance is given by $\epsilon_{97 \%}=2.565^{2} \pi \sigma_{p} \sigma_{T}$

Figure 5.7 shows the $99 \%$ longitudinal admittance as a function of aspect ratio, with optimal voltage tuning. The corresponding optimal $\alpha_{s}$ and $\lambda$ at those aspect ratios are shown in Fig. 5.8 and Fig. 5.9 respectively. The value of $\lambda$ with the maximum injection efficiency coincides with the value of $\lambda$ with the balanced condition for maximum stable phase-space area. 

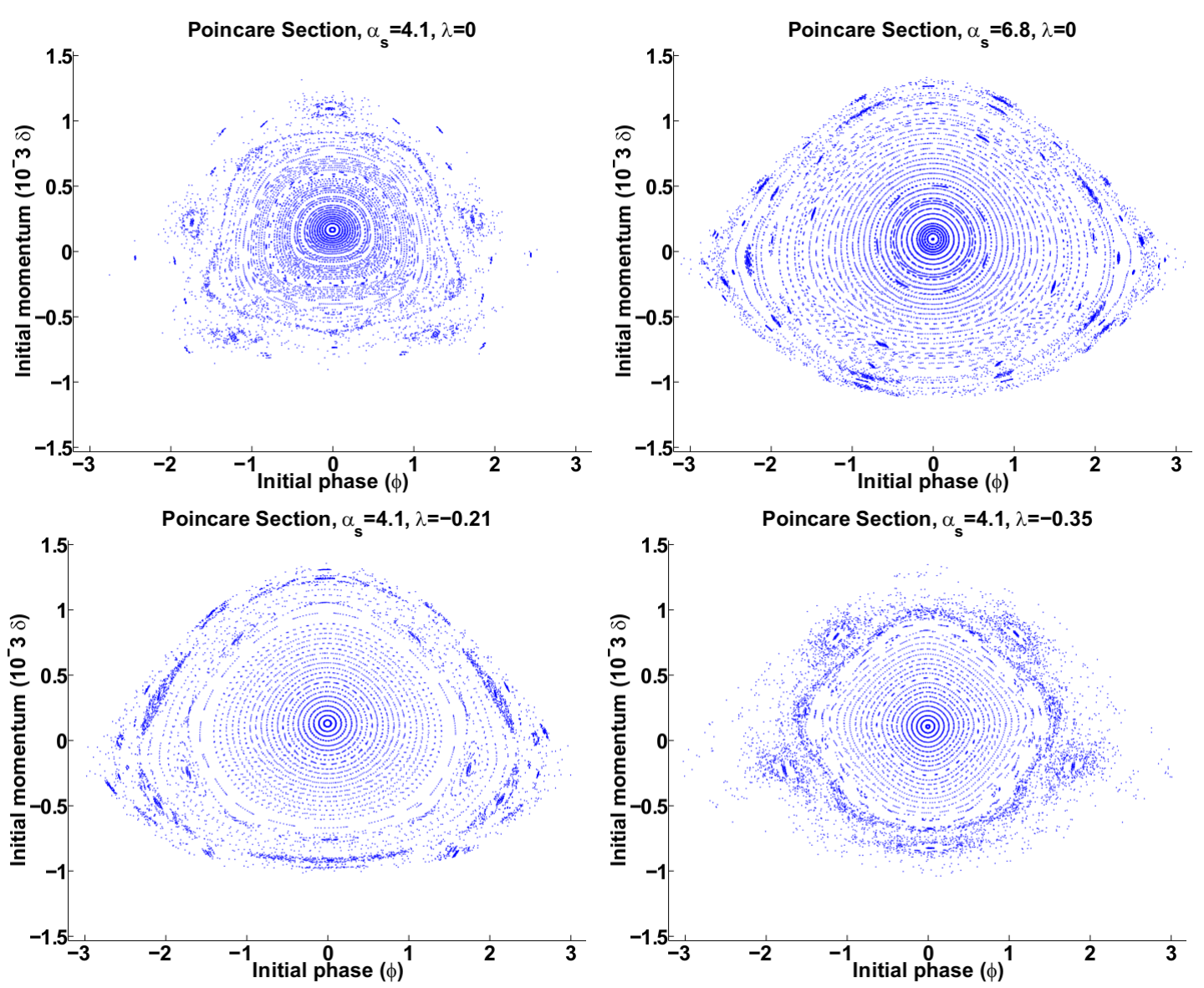

Figure 5.6: Selected Poincaré Maps for regular and harmonic slip-stacking. (a) Regular slipstacking with $\alpha_{s}$ corresponding to $100 \mathrm{kV}$ main $\mathrm{RF}$ and $1260 \mathrm{~Hz} \mathrm{RF}$ frequency separation. (b) Regular slip-stacking with $\alpha_{s}$ corresponding to $65 \mathrm{kV}$ main $\mathrm{RF}$ and $1380 \mathrm{~Hz} \mathrm{RF}$ frequency separation. c) Harmonic slip-stacking with balanced $\lambda$. (d) Harmonic slip-stacking with unbalanced $\lambda$. 
The aspect ratio is defined by the momentum spread divided by the temporal spread of the beam distribution. Operationally, the aspect ratio of the beam can be manipulated via bunch rotation in the Booster $[35,36]$. Recall, our measurement found the aspect ratio of the beam injected into the Recycler to be $\sim 1.45$ and the $99 \%$ emittance to be $0.25 \mathrm{eV} \cdot \mathrm{s}$ (see Table 4.1).

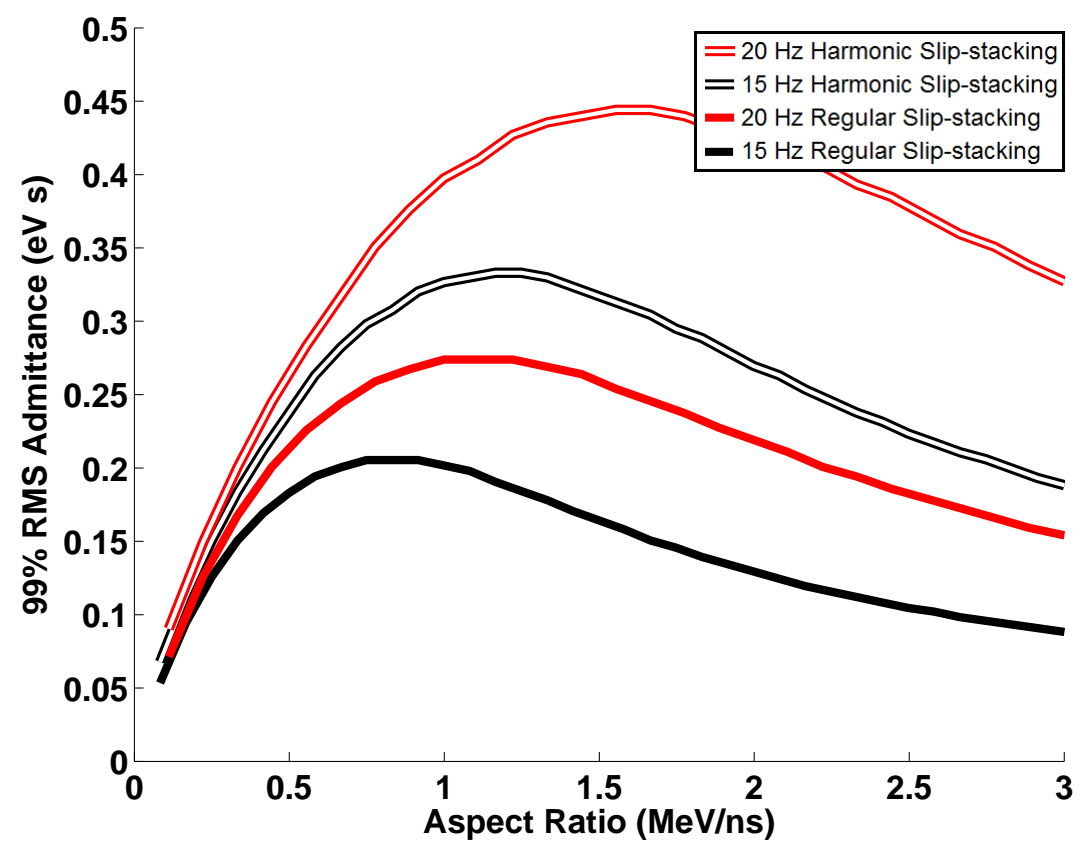

Figure 5.7: The 99\% admittance at 99\% efficiency (at an optimal $\alpha_{s}$ and $\lambda$ ) as a function of aspect ratio.

These results were obtaining using parameter values specific to slip-stacking in the Fermilab Recycler (see Table. 3.1), with the exception of the main RF voltage which was left unconstrained. In order to achieve the admittance shown in Figure 5.7, the main RF voltage must reach $250 \mathrm{kV}$ and the harmonic $\mathrm{RF}$ voltage must reach $70 \mathrm{kV}$. However this stable phase-space area far exceeds the requirements for slip-stacking operation with minimal loss. Fig. 5.10 shows the $99 \%$ admittance achievable with the main RF voltage limited to 100 $\mathrm{kV}$ and the harmonic RF voltage limited to $20 \mathrm{kV}$. 


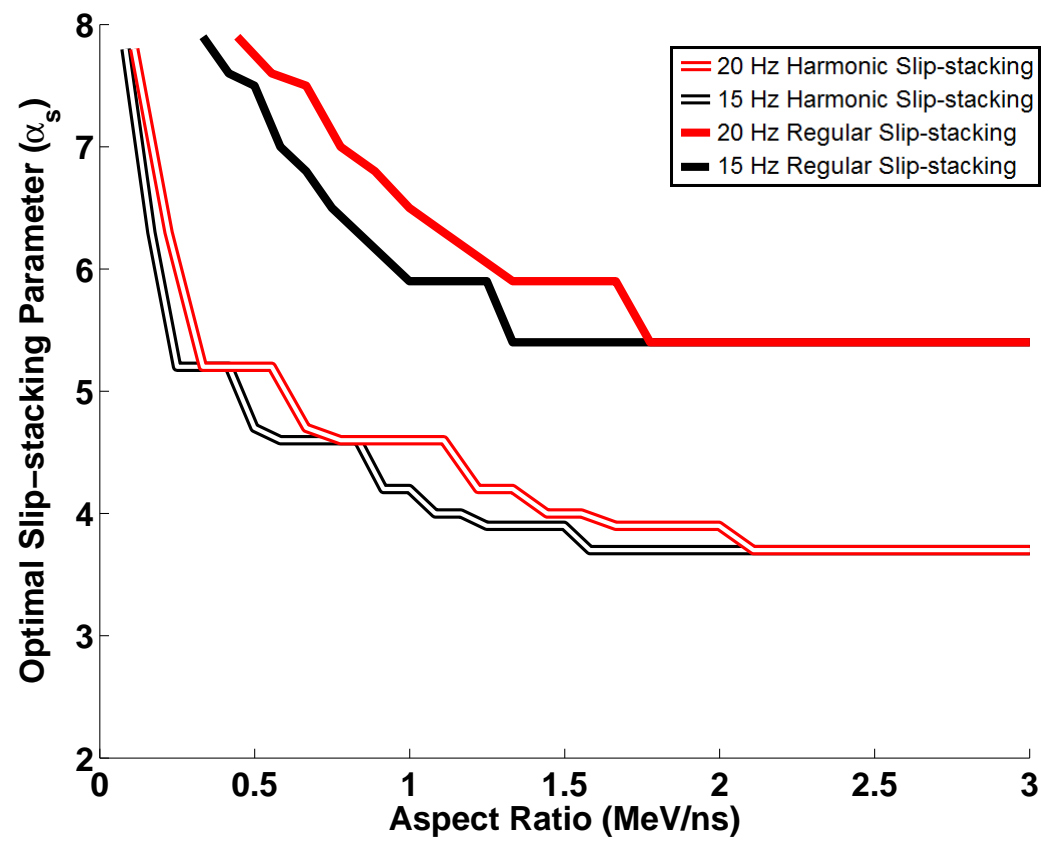

Figure 5.8: Optimal slip-stacking parameter $\alpha_{s}$ for $99 \%$ admittance (at $99 \%$ efficiency) as a function of aspect ratio.

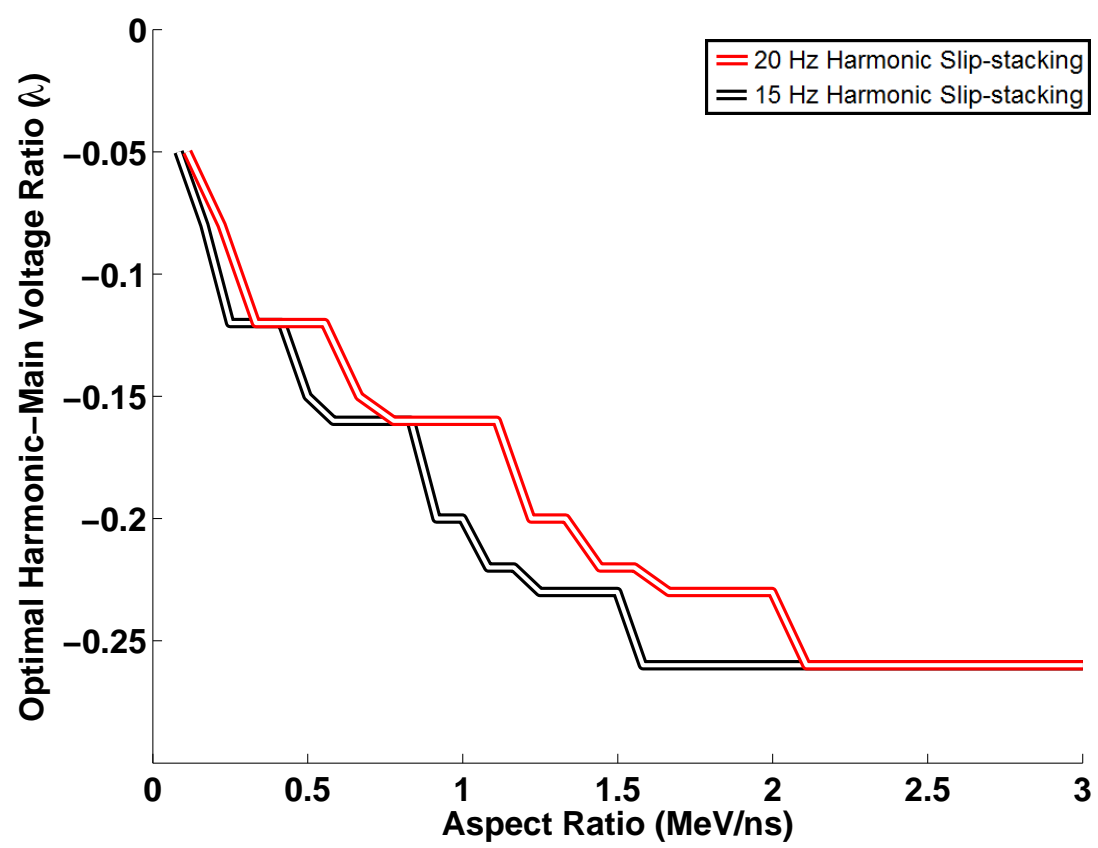

Figure 5.9: Optimal harmonic-main voltage ratio $\lambda$ for $99 \%$ admittance (at $99 \%$ efficiency) as a function of aspect ratio. 


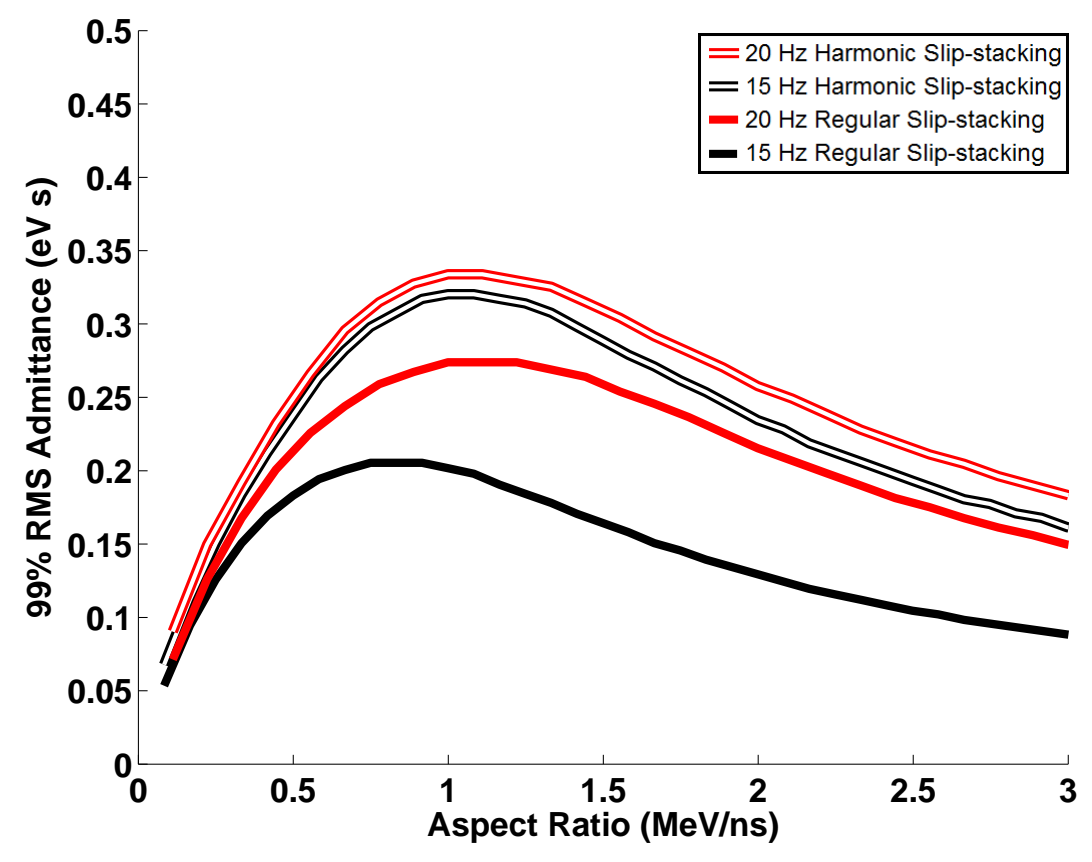

Figure 5.10: The $99 \%$ admittance at $99 \%$ efficiency as a function of aspect ratio. The choice of $\alpha_{s}$ and $\lambda$ is maximized under the constraint of $100 \mathrm{kV}$ main $\mathrm{RF}$ voltage and 20 $\mathrm{kV}$ harmonic $\mathrm{RF}$ voltage. 


\subsection{Mitigation of Emittance Growth}

In order for the harmonic RF to serve as a robust method for preventing particle loss, it is important to establish that this reduction of loss does not increase losses from other sources. The momentum spread of the beam is a critical specification, because the slipstacking beams must fit within the momentum aperture of the Recycler [77] at a given chromaticity $[42,78]$. The longitudinal emittance growth in the Recycler (before capture in the Main Injector) is another critical specification. This longitudinal emittance growth is a dilution effect caused by slip-stacking nonlinearies as well as any mismatch between the aspect ratio of the incoming beam and the RF bucket. Both the momentum spread and the longitudinal emittance of the beam in the Recycler contribute to the emittance of the captured beam in the Main Injector. In this section, we show the harmonic RF can reduce particle loss while decreasing the longitudinal emittance growth and maintaining the same momentum spread.

We also used numerical integration of the single-particle dynamics to analyze particle loss and longitudinal emittance growth during slip-stacking. The beam distribution fit from the tomography measurement was used as the initial beam distribution for our simulation (see Fig. 4.1 and Table 4.1). Each bin of the gaussian distribution was converted to macroparticle representing the intensity of that bin. The trajectories of these macroparticles were numerically integrated using Eq. 5.1 for 32000 revolutions. Then the lost particles, particles with phase greater than $3 \pi / 2$, were removed. The initial and final RMS momentum spread and RMS temporal spread were calculated from the remaining particles. The simulation was repeated under variation of the main $\mathrm{RF}$ voltage and the harmonic RF voltage parameters (with the remaining parameters taken from Table 3.1). Beam capture in the Main Injector was not part of this analysis.

Fig. 5.11 shows the particle loss, Fig. 5.12 shows the longitudinal emittance growth, and 
Fig. 5.13 shows the momentum spread growth. In Fig. 5.11 and Fig. 5.12, the balanced condition can be seen as a diagonal line and is consistent with Eq. 5.5. If the main RF voltage is too low or too high there is a mismatch in aspect ratio between the beam and bucket which results in increased losses and emittance growth. In Fig. 5.13, it is clear that the dominant effect on the momentum spread is the aspect ratio of the bucket, but the resonance-canceling and bucket-matching effects also influence the result.

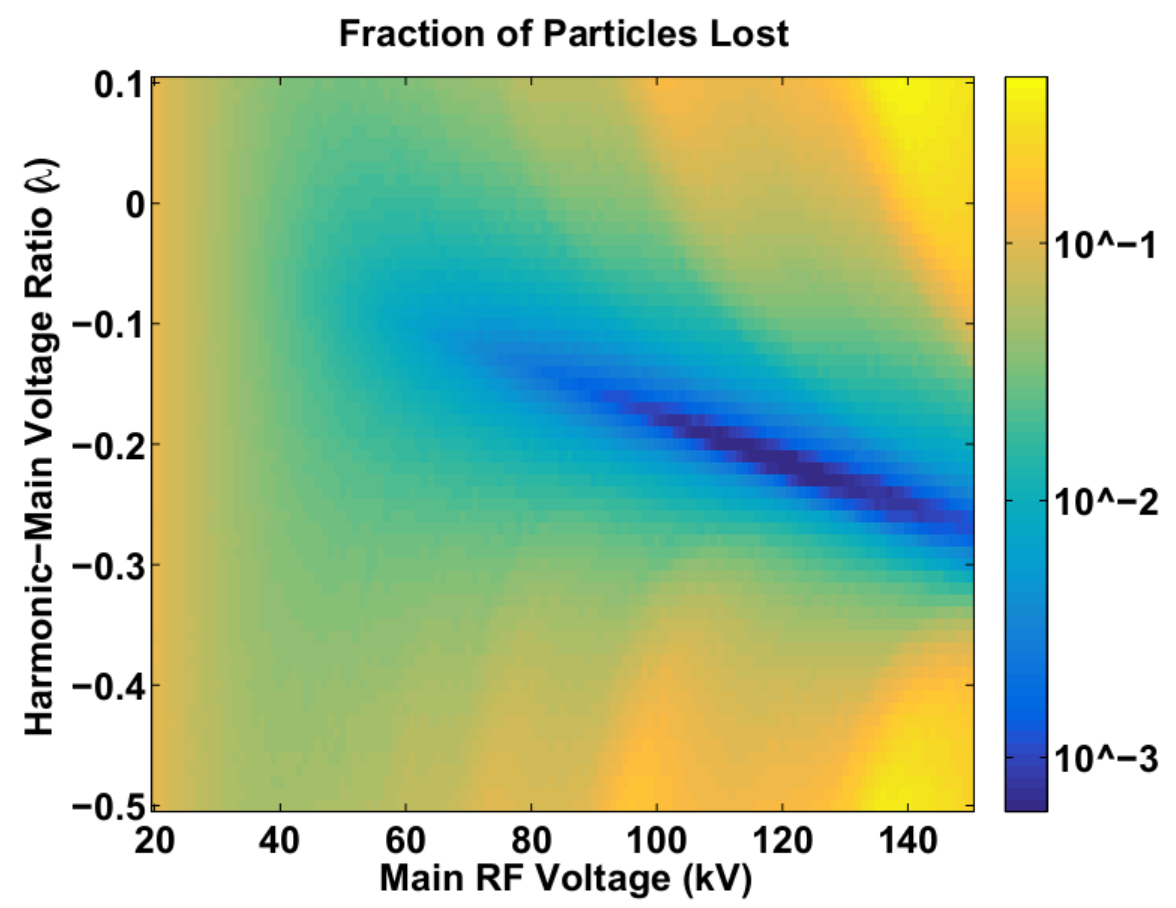

Figure 5.11: Particle loss as a function of main RF voltage and $\lambda$.

Fig. 5.14 and Fig. 5.15 are correlation plots that show the relationship between particle loss, emittance growth, and momentum spread. Without harmonic RF, the minimal loss condition and the minimum emittance condition coincide with a moderate momentum spread. The harmonic RF opens a new tuning range with lower loss, lower emittance, or a combination thereof. If the longitudinal distribution falls off more sharply then a gaussian, then the Pareto front is more L-shaped then in Fig. 5.14 and Fig. 5.15.

Table 5.1 compares the loss rate, emittance growth and momentum growth for slip- 


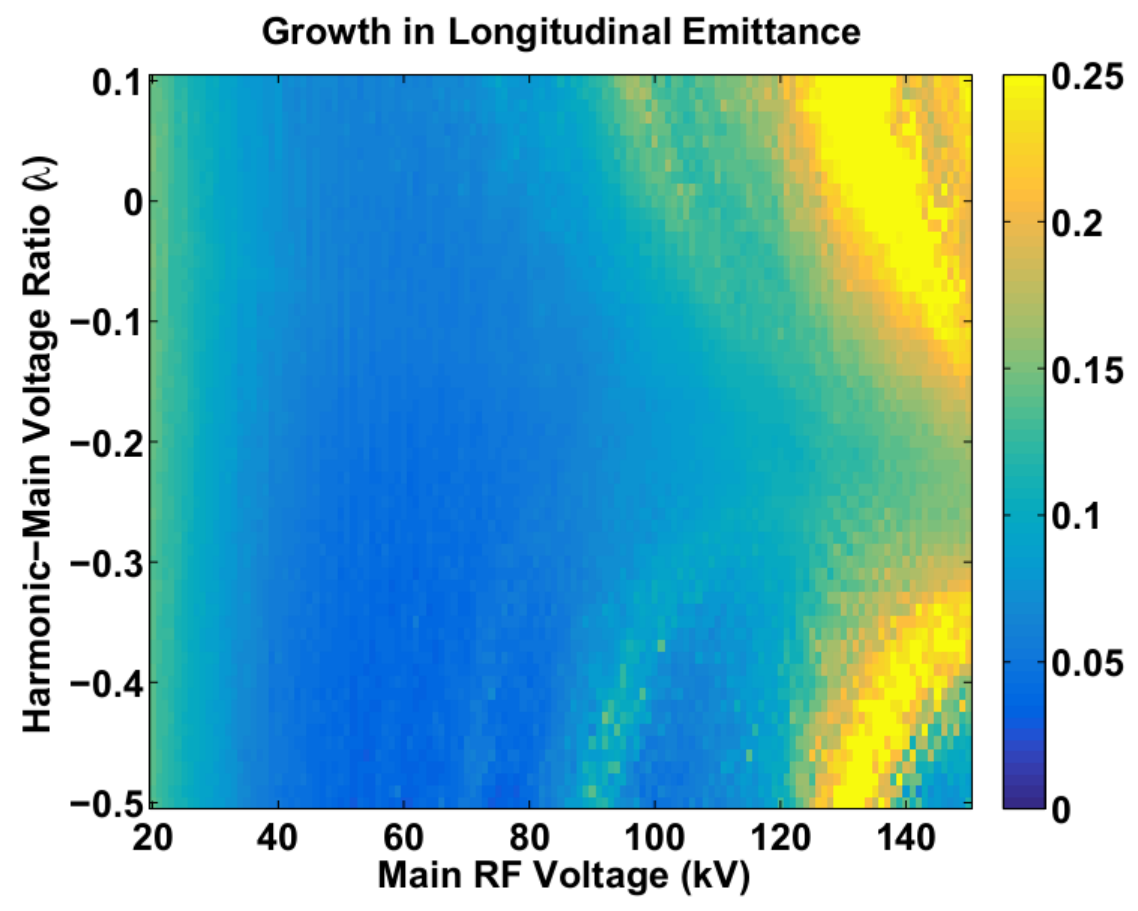

Figure 5.12: Longitudinal emittance growth as a function of main RF voltage and $\lambda$.

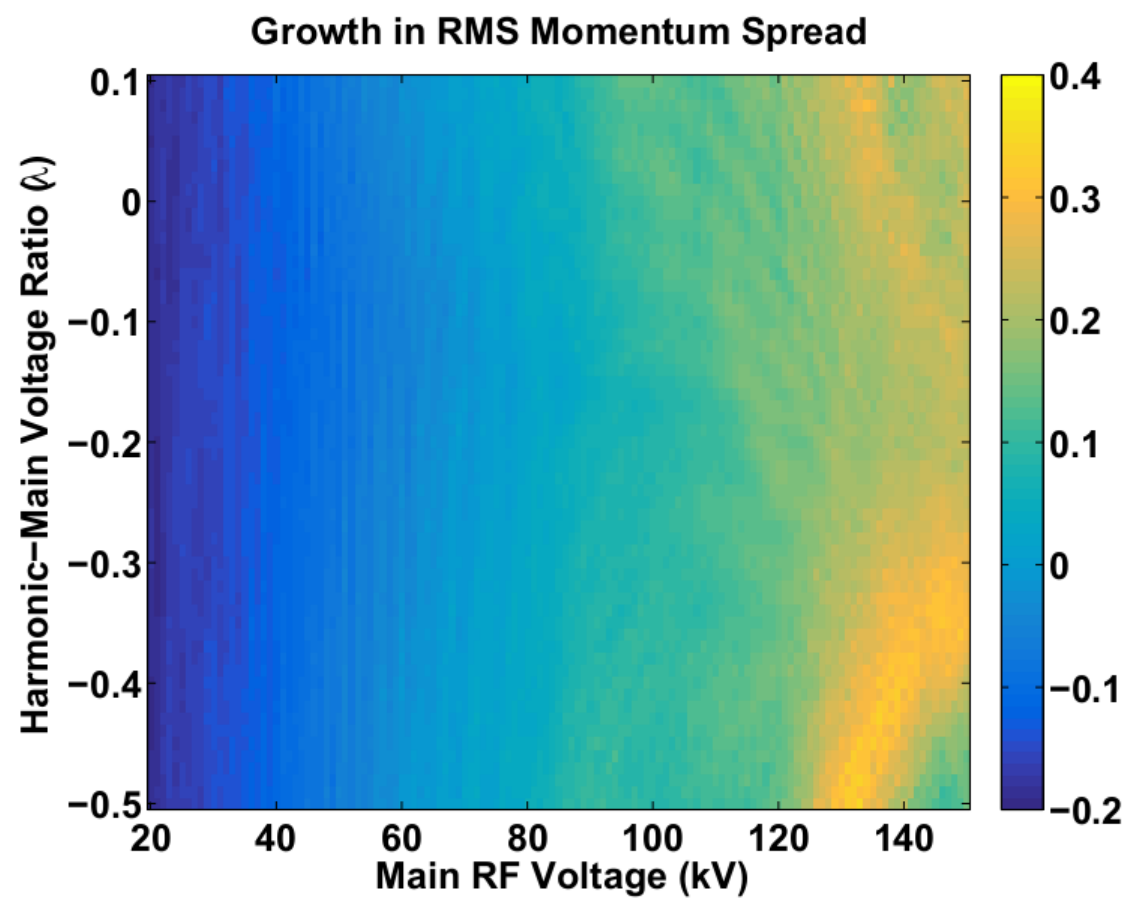

Figure 5.13: Growth in momentum spread as a function of main RF voltage and $\lambda$. 


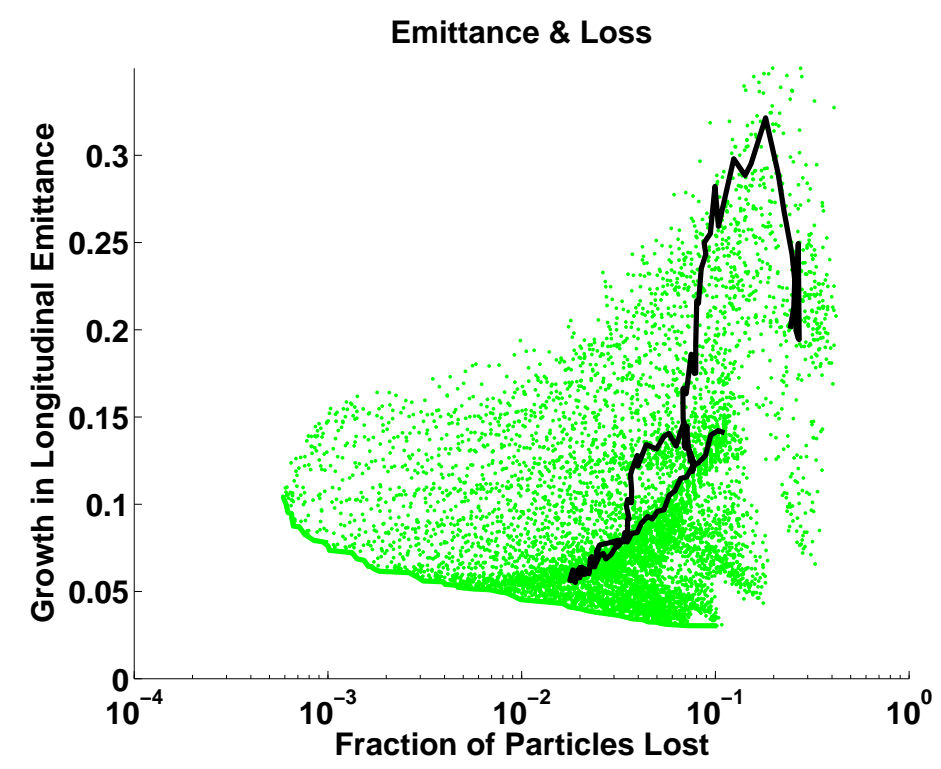

Figure 5.14: Longitudinal emittance growth as a function of particle loss. The thick black line shows the case where there is no harmonic $R F$ and the main $R F$ is varied. The green shows the cases with harmonic RF (points) and the corresponding Pareto front (line).

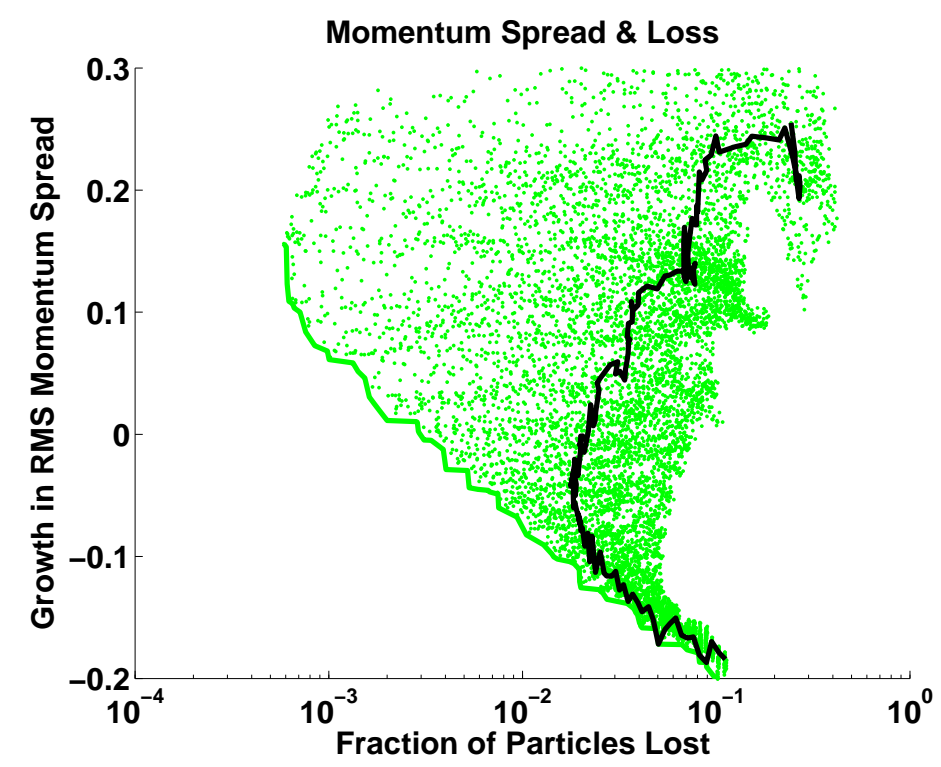

Figure 5.15: Growth in momentum spread as a function of particle loss. The thick black line shows the case where there is no harmonic $\mathrm{RF}$ and the main $\mathrm{RF}$ is varied. The green shows the cases with harmonic RF (points) and the corresponding Pareto front (line). 


\begin{tabular}{|l|c|c|c|c|c|}
\hline Case & Loss & Emittance & Momentum Spread & Main Voltage & Harmonic Voltage \\
\hline A & $1.8 \%$ & $+5.6 \%$ & $-4.3 \%$ & $62 \mathrm{kV}$ & $0 \mathrm{kV}$ \\
$\mathrm{B}$ & $0.53 \%$ & $+5.5 \%$ & $-4.4 \%$ & $66 \mathrm{kV}$ & $-7.3 \mathrm{kV}$ \\
$\mathrm{C}$ & $0.31 \%$ & $+5.6 \%$ & $-0.5 \%$ & $78 \mathrm{kV}$ & $-11 \mathrm{kV}$ \\
$\mathrm{D}$ & $0.095 \%$ & $+7.8 \%$ & $+7.6 \%$ & $100 \mathrm{kV}$ & $-18 \mathrm{kV}$ \\
$\mathrm{E}$ & $1.8 \%$ & $+4.1 \%$ & $-6.1 \%$ & $61 \mathrm{kV}$ & $-12 \mathrm{kV}$ \\
$\mathrm{F}$ & $1.8 \%$ & $+6.3 \%$ & $-10 \%$ & $47 \mathrm{kV}$ & $-3.3 \mathrm{kV}$ \\
\hline
\end{tabular}

Table 5.1: Several cases loss rate, emittance growth and momentum growth based on simulation results. A: Slip-stacking in status quo. B: Keep emittance and momentum spread, but minimize loss. C: Keep emittance, but minimize loss. D: Minimize loss. E: Keep loss, but minimize emittance. F: Keep loss, but minimize momentum spread.

stacking with and without harmonic RF. In the status quo (Case A), the loss rate is calculated to be $1.8 \%$ and the optimal main $\mathrm{RF}$ voltage to be $62 \mathrm{kV}$. Those number are consistent with recent experimental studies described in the previous chapter. Case D indicates that installing a $20 \mathrm{kV}$ harmonic cavity could cut those losses by a factor $\sim 20$. Case $\mathrm{D}$ also entails at $\sim 12 \%$ increase in momentum spread over the status quo, primarily caused by the higher main RF voltage. Case B indicates losses can be cut by factor of $\sim 3$ without changing the momentum spread or emittance, and Case $\mathrm{C}$ presents an intermediate scenario. Case $\mathrm{E} \& \mathrm{~F}$ indicate that the loss rate can also be maintained while reducing emittance (by $1 \%$ relative to status quo) or reducing momentum spread (by $-6 \%$ relative to status quo).

Recall that the experimental studies of slip-stacking described in the previous chapter have found a $0.5 \% \pm 0.2 \%$ loss-rate of particles from Booster bunch rotation. If a harmonic RF cavity were installed then the Recycler could capture a beam with a higher aspect ratio and Booster bunch rotation could be retuned for lower losses. 


\subsection{Chapter Conclusion}

We introduced a harmonic cavity which cancels slip-stacking resonances and dramatically increases the dynamical stability of slip-stacking. We derived and verified a model to predict the dependence of the optimal harmonic $R F$ voltage on the main $R F$ voltage. We characterized the stable slip-stacking phase-space area for any combination of accelerator parameters, with and without harmonic RF. We calculated the longitudinal admittance as a function of longitudinal aspect ratio and found the corresponding optimization of parameters $\alpha_{s}$ and $\lambda$. We calculated loss-rate, emittance growth, and momentum spread growth of a realistic beam during slip-stacking. From simulation, particle loss can be reduced by a factor of $\sim 20$ with a $2 \%$ increase in longitudinal emittance growth or loss can be reduced by a factor of $\sim 3$ with no increase in longitudinal emittance growth or momentum spread growth. The new harmonic cavity may also enable slip-stacking to be feasible at the higher beam intensities prescribed by a hypothetical PIP-III RCS.

\subsection{Dissertation Conclusion}

In this dissertation, I have analyzed the dynamics of slip-stacking analytically, numerically, and experimentally. That insight into slip-stacking dynamics led me to propose a harmonic RF cavity to cancel nonlinear slip-stacking resonances and increase the stable phase-space area. The simulation results indicated that the harmonic RF cavity would dramatically reduce slip-stacking losses.

I designed an experimental study to measure the losses in the Recycler directly attributable to the single-particle longitudinal dynamics of slip-stacking. The experimental study of the RF frequency separation showed that a $20 \mathrm{~Hz}$ Booster cycle-rate would reduce slip-stacking losses to a rate comparable to the single-RF case. By verifying the simulation

results, the experimental results imply that the harmonic RF cavity will also have a direct 
impact on the operationally observed particle losses.

A $106 \mathrm{MHz} 20 \mathrm{kV}$ RF cavity should be installed in the Fermilab Recycler to reduce losses ahead of the PIP-II upgrade. The upgrade of the Booster cycle-rate to $20 \mathrm{~Hz}$ may not be necessary to enhance the stability of slip-stacking. The anticipated reduction in slip-stacking losses from the harmonic RF cavity enables slip-stacking to be considered for the PIP-III era. The PIP-III era Recycler may require further work to address losses from betatron resonances, head-tail instabilities, electron cloud instabilities, and Booster bunch-rotation. 


\section{A POINCARÉ MAPS FOR SLIP-STACKING}

This appendix shows the Poincaré maps of the higher slip-stacking bucket for values of $\alpha_{s}$ from 2.3 to 8.0 in descending 0.1 increments. Recall that the Poincaré maps show the coordinates of a phase-space trajectories at $2 \pi / \omega_{\phi}$ intervals. The following differential equation is used:

$$
\dot{\phi}=2 \pi f_{r e v} h \eta \delta, \dot{\delta}=f_{r e v} V_{\delta}\left[\sin (\phi)+\sin \left(\phi-\omega_{\phi} t\right)\right]
$$

Where $\phi$ is the phase of the particle relative to the RF cavity and $\delta$ is the fractional deviation from a reference momentum.
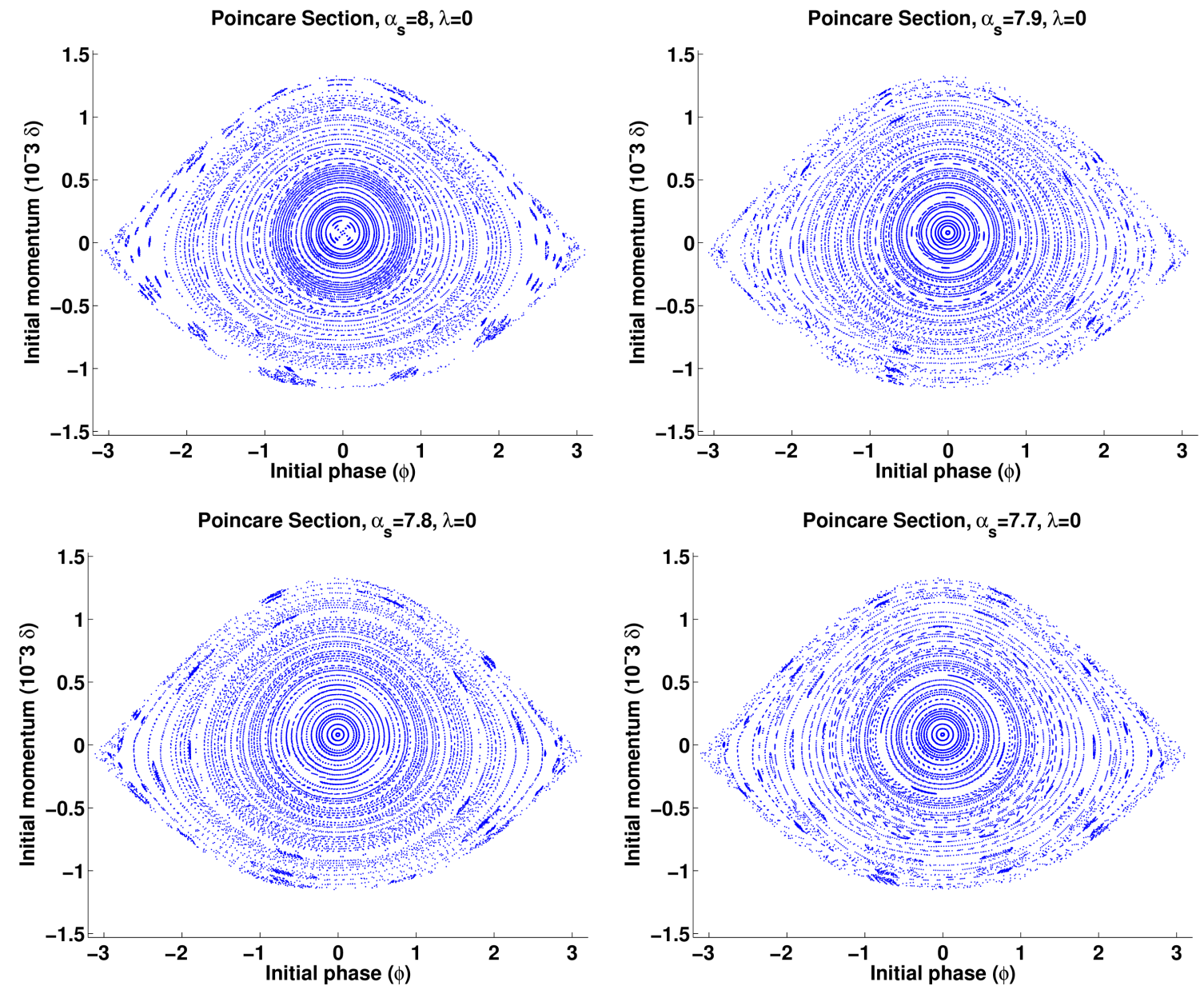

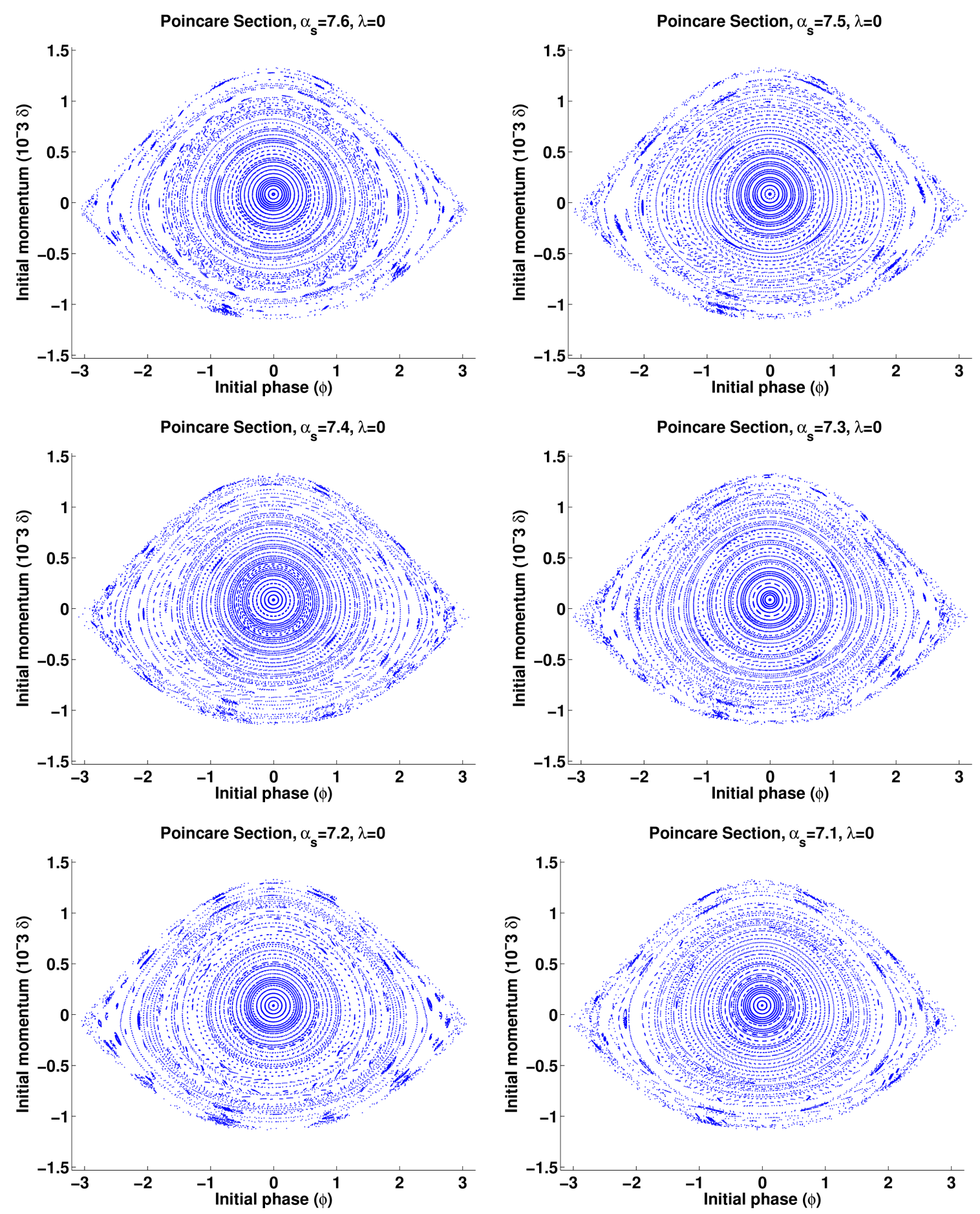

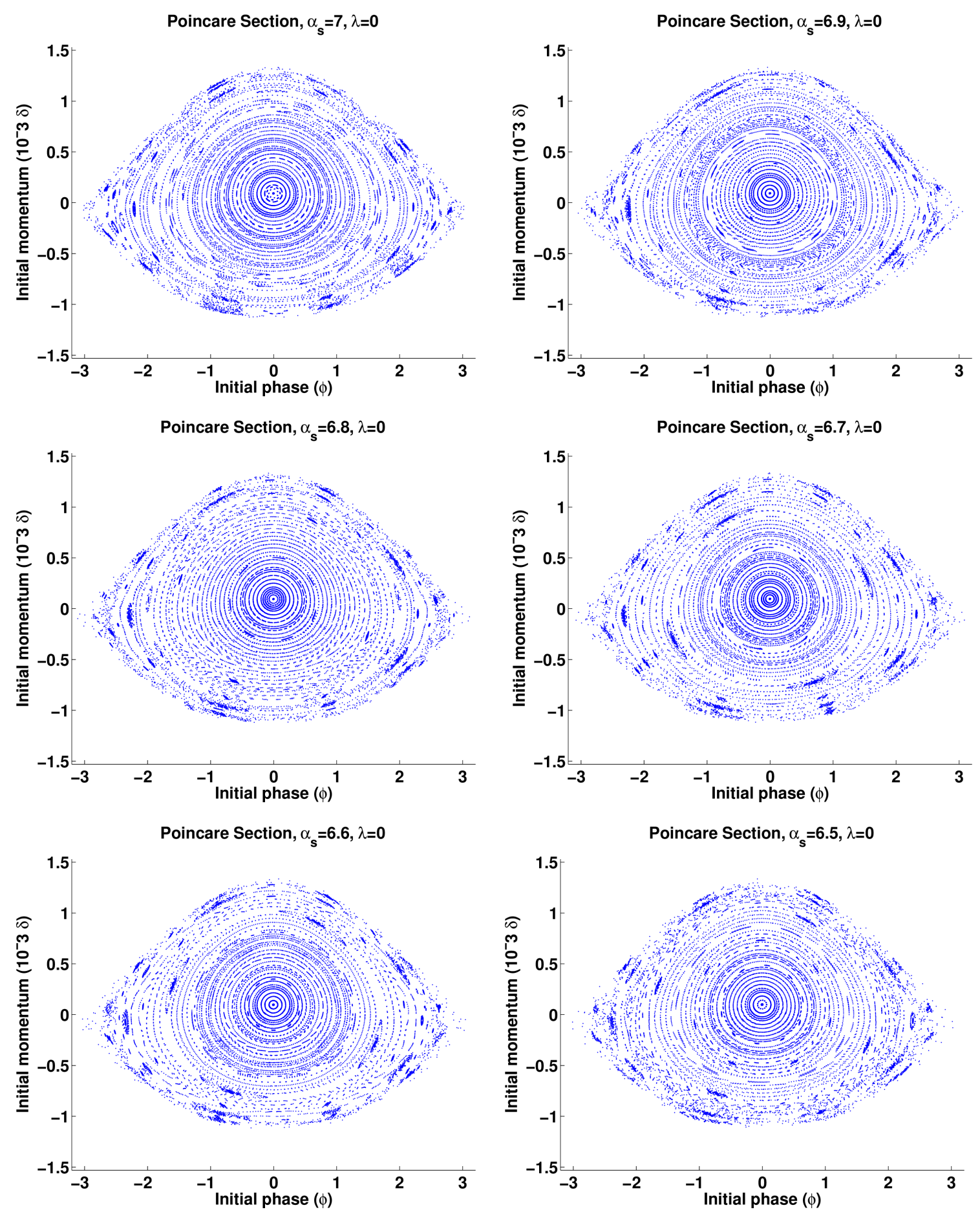

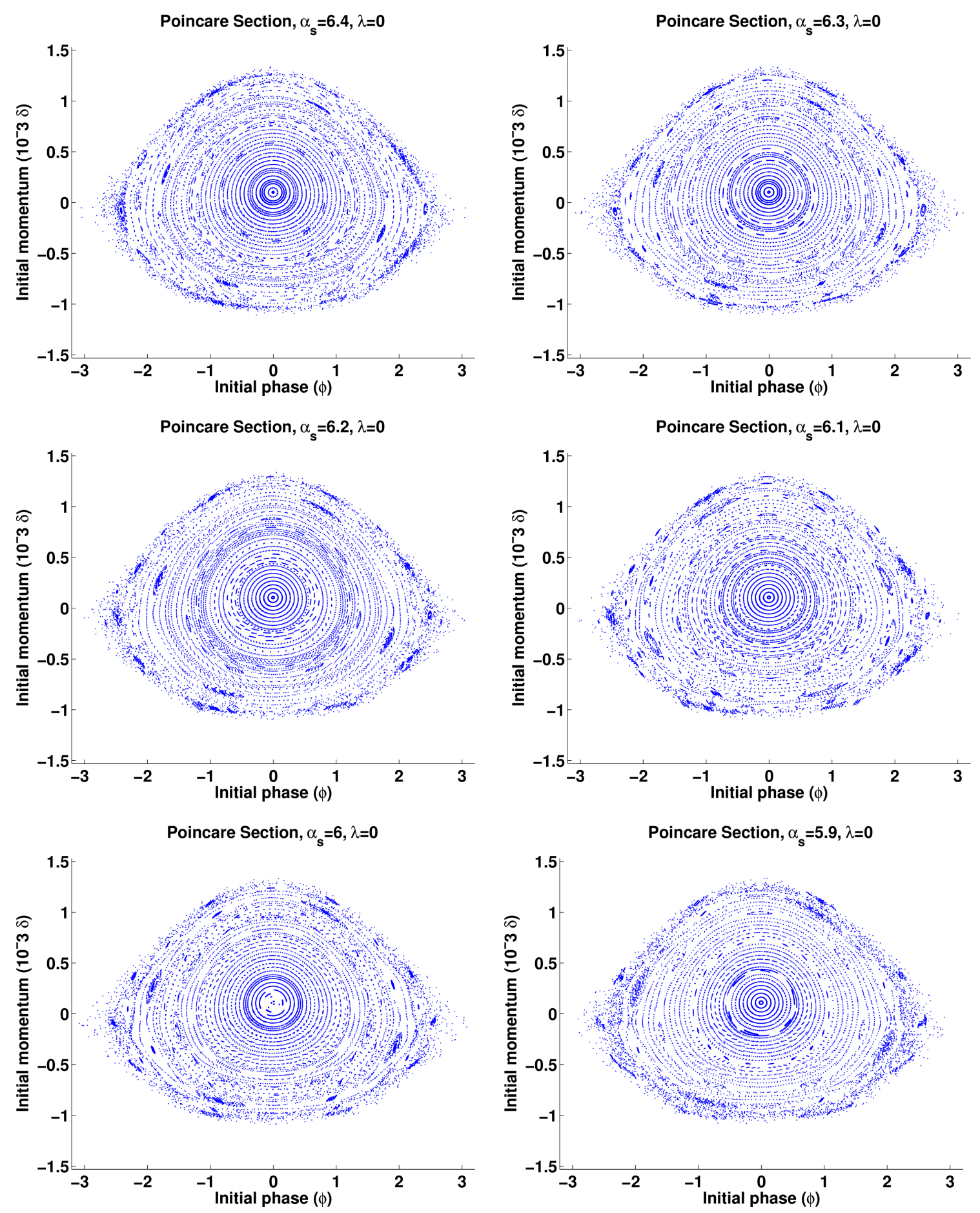

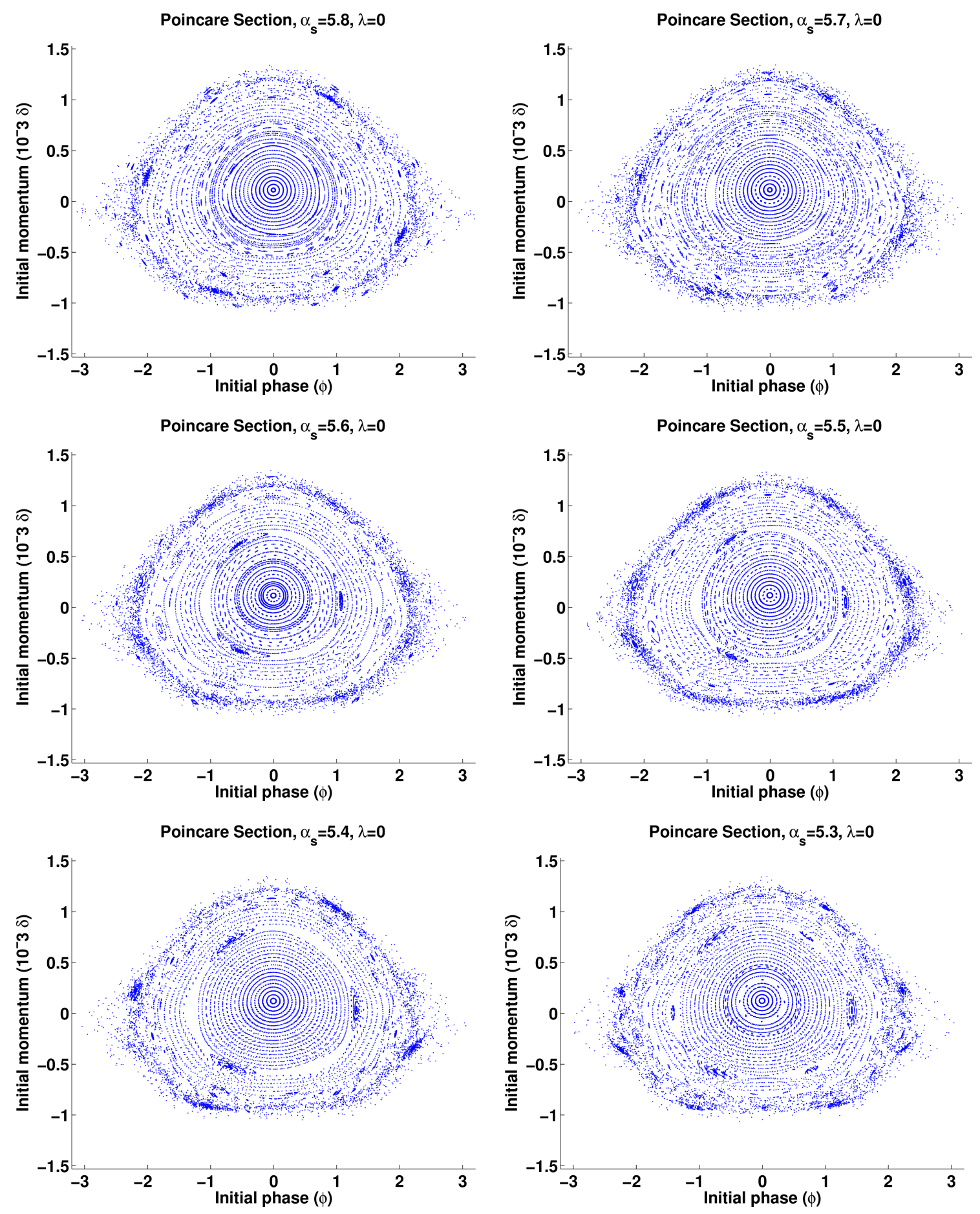

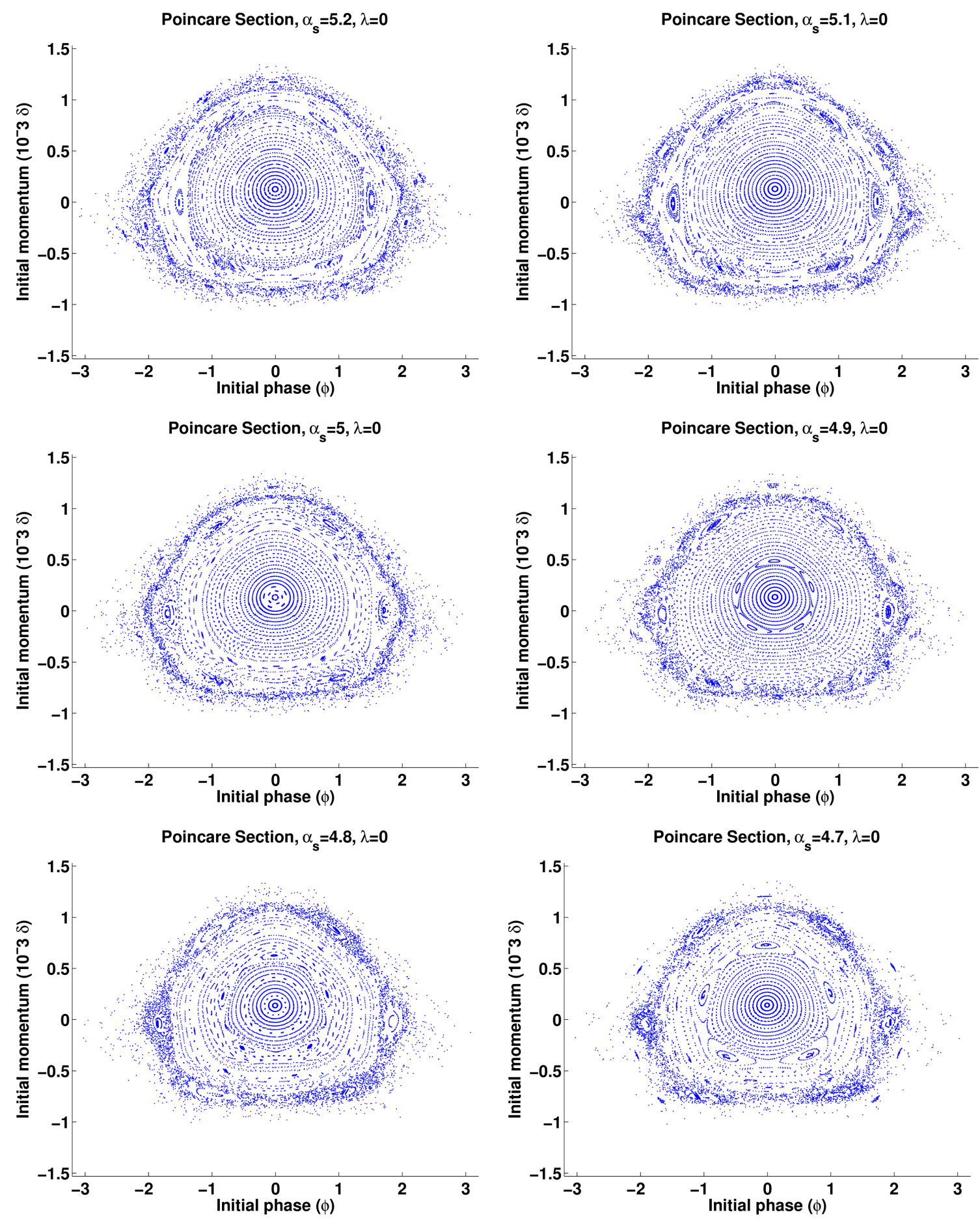

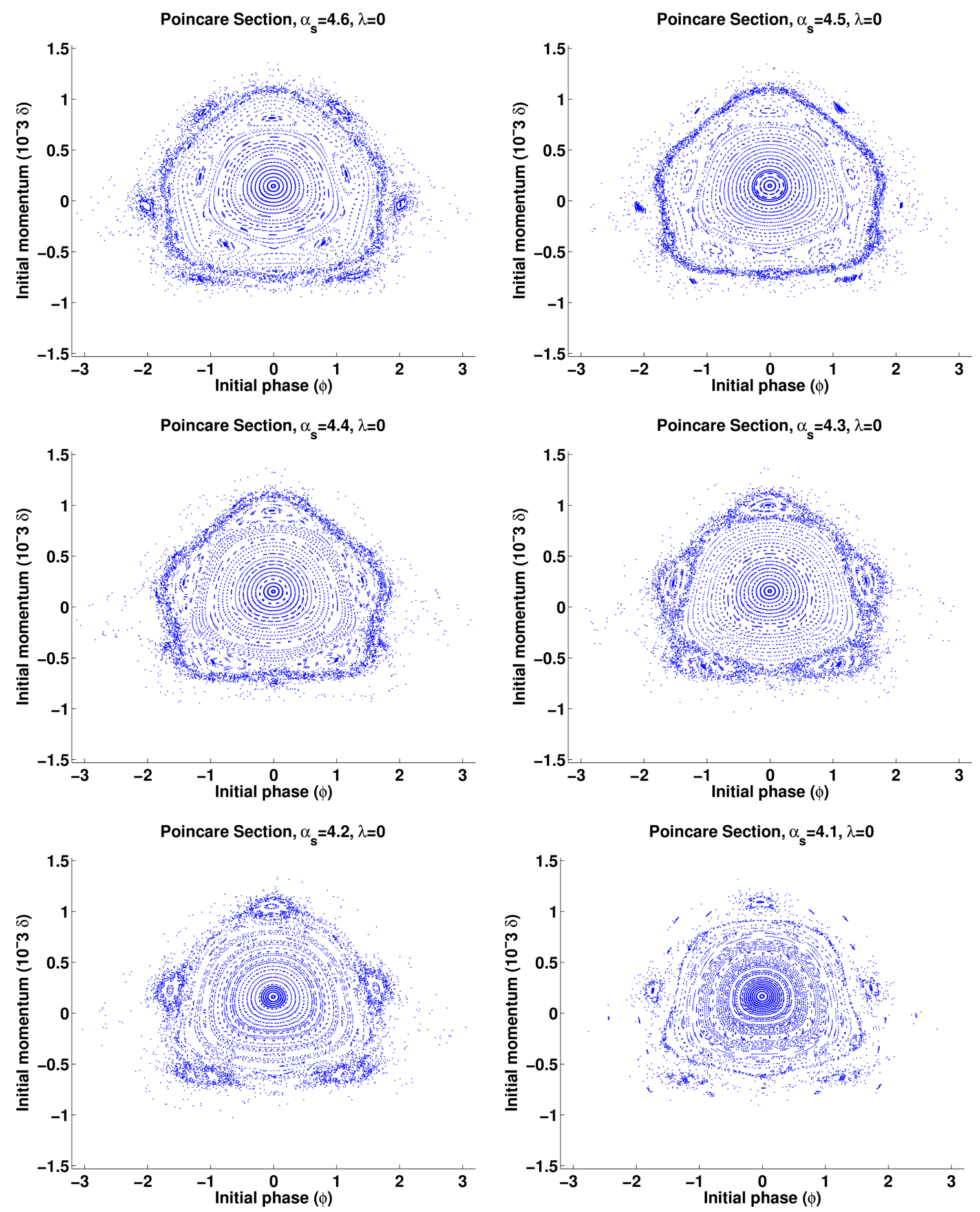

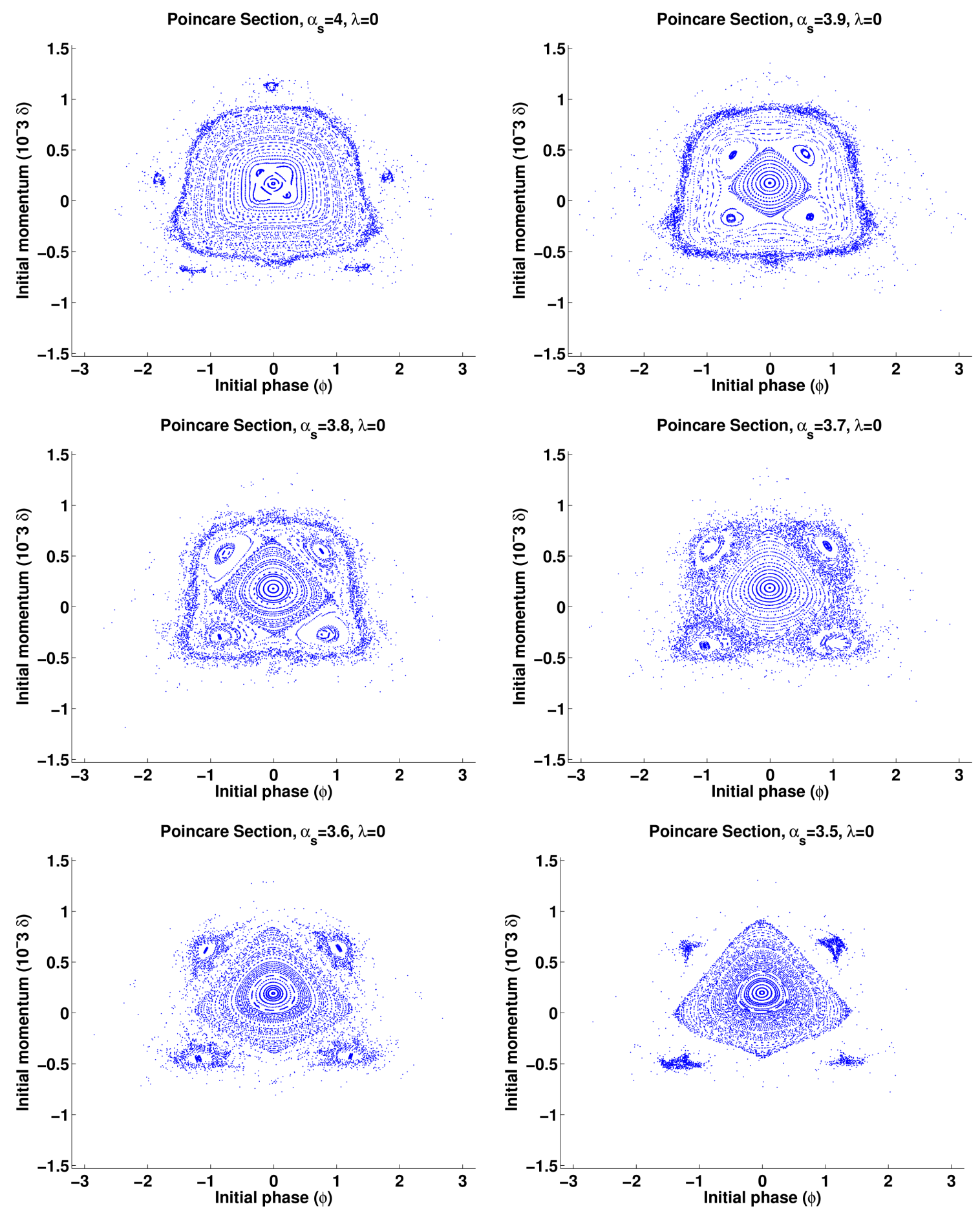

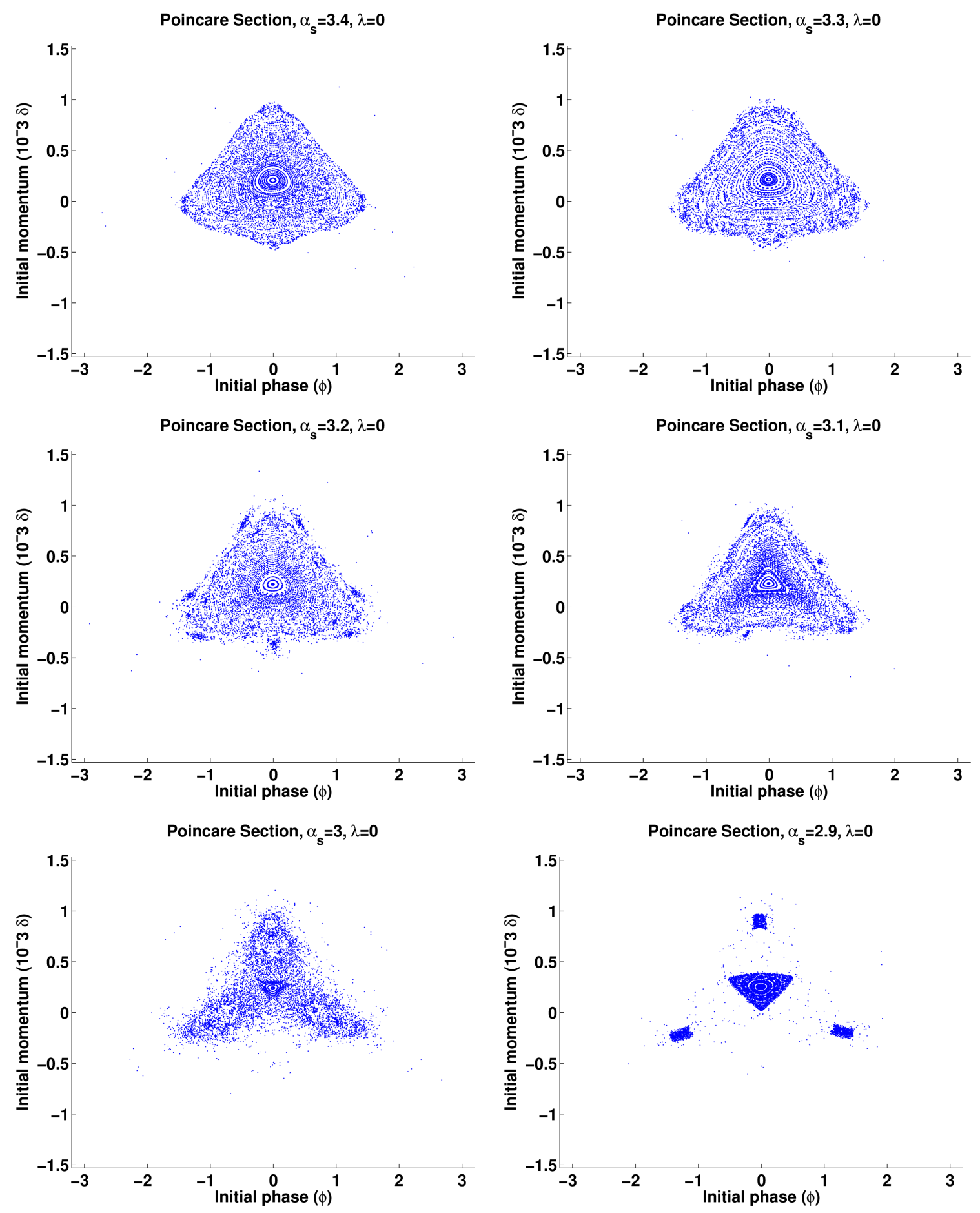

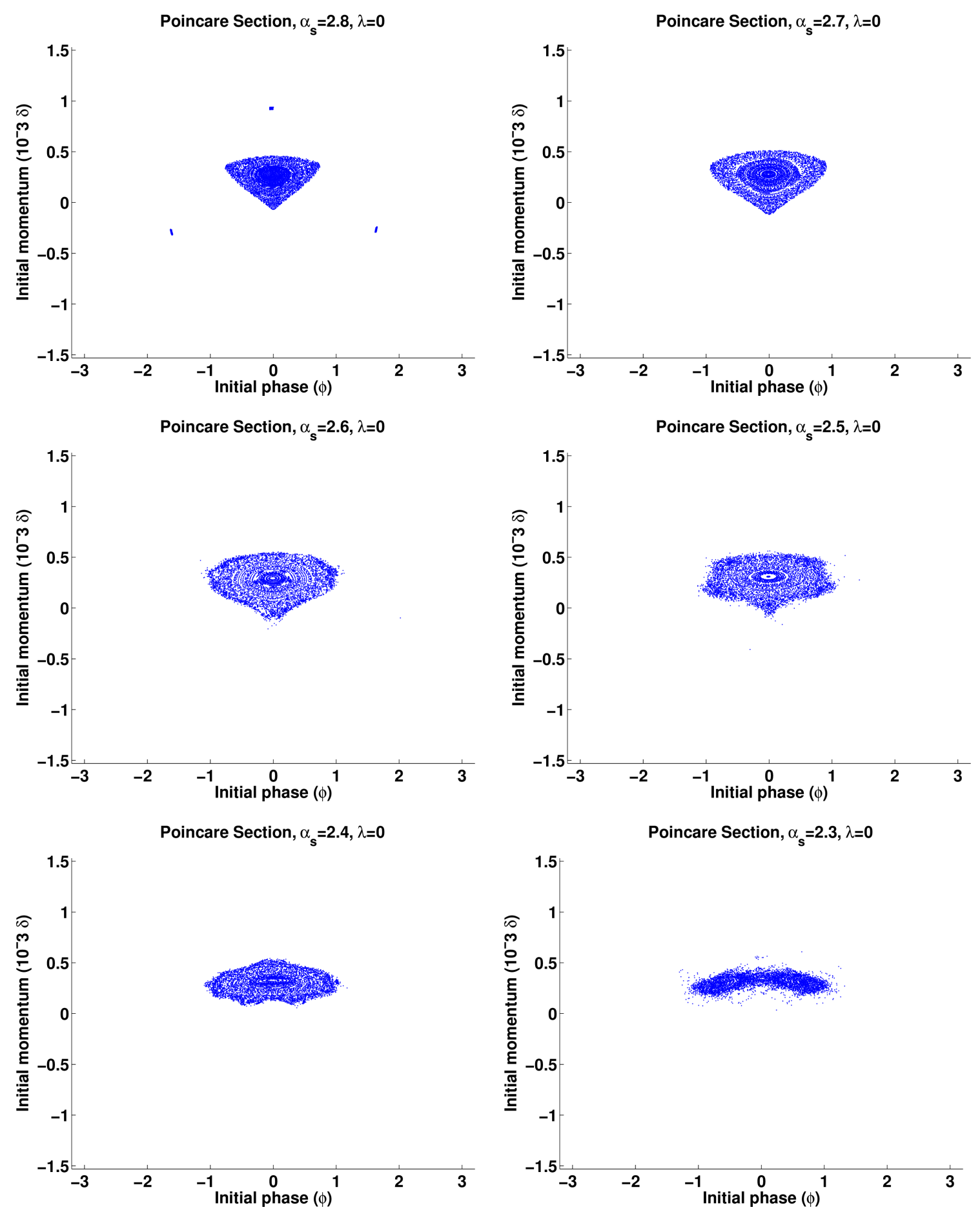


\section{B SLIP-STACKING PERTURBATIVE SOLUTION}

In Chapter 3 of this dissertation we show that the second-order equation of motion for a single particle in a slip-stacking bucket is given by Eq. 3.3 and the perturbative solution is given by Eq. 3.6. Recall also, that the coefficients $A_{n}$ are of order $\alpha_{s}^{-2 n}$ and $B_{m, n}$ are of

order $\rho^{m} \alpha_{s}^{-2|n|}$ except the $B_{2 k, 0}$ coefficients which are of the order $\rho^{2 k} \alpha_{s}^{-2}$. The coefficient $B_{1,0}$ is defined to be equal to $\rho$. The parameters $\rho$ and $\psi$ are set by initial conditions.

For clarity, we adopt a short-hand notation for the oscillatory terms as follows:

$$
\begin{aligned}
& \sin \left[m(1+\sigma) \omega_{s} t+n \omega_{\phi} t+m \psi\right] \equiv s_{m, n}, \\
& \cos \left[m(1+\sigma) \omega_{s} t+n \omega_{\phi} t+m \psi\right] \equiv c_{m, n} .
\end{aligned}
$$

In this appendix, we explicitly obtain the perturbative solution up to order $\alpha_{s}^{-4}$ and $\rho \alpha_{s}^{-2}$ (or equivalently, all coefficients up to order $\alpha_{s}^{-5}$ with $\rho \sim \alpha_{s}^{-3}$ ). Therefore, we use the coefficients $A_{1}, A_{2}, \rho, B_{1,1}$ and $B_{1,-1}$; all other coefficients are neglected at this precision. We start by assuming a solution form and will demonstrate it to be self-consistent:

$$
\phi=A_{1} s_{0,1}+A_{2} s_{0,2}+\rho s_{1,0}+B_{1,1} s_{1,1}+B_{1,-1} s_{1,-1} .
$$

It will be sufficient to substitute this expression into the form of Eq. 3.6 expanded up to second order in $\phi$ :

$$
\omega_{\phi}^{-2} \ddot{\phi}=-\alpha_{s}^{-2}\left[\phi\left(1+c_{0,1}\right)-s_{0,1}+\frac{1}{2} \phi^{2} s_{0,1}\right]
$$

Here we have divided both sides by $\omega_{\phi}^{2}$ to make the order of the perturbation terms more explicit.

Next we can substitute the solution given in Eq. B.2 into Eq. B.3 to calculate the 
coefficients. We write out the left-hand side (LHS):

$$
\begin{aligned}
& -A_{1} s_{0,1}-4 A_{1} s_{0,2} \\
& -\alpha_{s}^{-2}(1+\sigma)^{2} \rho s_{1,0} \\
& -\left[\alpha_{s}^{-1}(1+\sigma)+1\right]^{2} B_{1,1} s_{1,1} \\
& -\left[\alpha_{s}^{-1}(1+\sigma)-1\right]^{2} B_{1,-1} s_{1,-1}=
\end{aligned}
$$

We write out the right-hand side (RHS):

$$
\begin{aligned}
& =-\alpha_{s}^{-2}\left(A_{1} s_{0,1}+A_{1} s_{0,1} c_{0,1}+\frac{1}{2} A_{1}^{2} s_{0,1} s_{0,1} s_{0,1}+A_{2} s_{0,2}+A_{2} s_{0,2} c_{0,1}-s_{0,1}\right. \\
& +\frac{1}{2} \not A_{2}^{\not 2} s_{0,2} s_{0,2} s_{0,1}+A_{1} A_{2} s_{0,1} s_{0,2} s_{0,1}+\rho s_{1,0}+\rho s_{1,0} c_{0,1} \\
& +B_{1,1} s_{1,1}+B_{1,1} s_{1,1} c_{0,1}+B_{1,-1} s_{1,-1}+B_{1,-1} s_{1,-1} c_{0,1}
\end{aligned}
$$

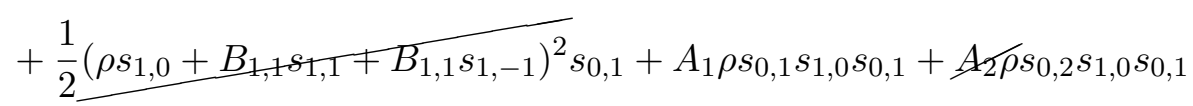

$$
\begin{aligned}
& +\underline{A}_{1} B_{1,1} s_{0,1} s_{1,1} s_{0,1}+A_{1} B_{1,-1} s_{0,1} s_{1,-1} s_{0,1} \\
& \left.+A_{2} B_{1,1} s_{0,2} s_{1,1} s_{0,1}+A_{2} B_{1,-1} s_{0,2} s_{1,-1} s_{0,1}\right) \text {. }
\end{aligned}
$$

where the crossed-out terms are higher order than the precision of this analysis. We rewrite the RHS without these immediately negligible terms, using the trigonometric product-tosum rules, and grouping by the oscillatory term:

$$
\begin{aligned}
=-\alpha_{s}^{-2}[ & \left(A_{1}-1+\frac{1}{2} A_{2}+\frac{3}{8} A_{1}^{2}\right) s_{0,1} \\
& +\left(\frac{1}{2} A_{1}+A_{2}\right) s_{0,2}+\left(\frac{1}{2} A_{2}-\frac{1}{8} A_{1}^{2}\right) s_{0,3} \\
& +\left(\rho+\frac{1}{2} B_{1,1}+\frac{1}{2} B_{1,-1}+\frac{1}{2} A_{1} \rho\right) s_{1,0} \\
& +\left(\frac{1}{2} \rho+B_{1,1}\right) s_{1,1}+\left(\frac{1}{2} \rho+B_{1,-1}\right) s_{1,-1} \\
& \left.+\left(\frac{1}{2} B_{1,1}-\frac{1}{4} A_{1} \rho\right) s_{1,2}+\left(\frac{1}{2} B_{1,-1}-\frac{1}{4} A_{1} \rho\right) s_{1,-2}\right]
\end{aligned}
$$

We then equate Eq. B.4 with Eq. B.6 for all time. Each oscillatory term corresponds to its 
own equation:

$$
\begin{gathered}
-A_{1}=-\alpha_{s}^{-2}\left(A_{1}-1+\frac{1}{2} A_{2}+\frac{3}{8} A_{1}^{2}\right) . \\
-4 A_{2}=-\alpha_{s}^{-2}\left(\frac{1}{2} A_{1}+A_{2}\right) . \\
-\alpha_{s}^{-2}(1+\sigma)^{2} \rho=-\alpha_{s}^{-2}\left(\rho+\frac{1}{2} B_{1,1}+\frac{1}{2} B_{1,-1}+\frac{1}{2} A_{1} \rho\right) . \\
-\left[\alpha_{s}^{-1}(1+\sigma)+1\right]^{2} B_{1,1}=-\alpha_{s}^{-2}\left(\frac{1}{2} \rho+B_{1,1}\right) . \\
-\left[\alpha_{s}^{-1}(1+\sigma)-1\right]^{2} B_{1,-1}=-\alpha_{s}^{-2}\left(\frac{1}{2} \rho+B_{1,-1}\right) . \\
0=-\alpha_{s}^{-2}\left(\frac{1}{2} A_{2}-\frac{1}{8} A_{1}^{2}\right) . \\
0=-\frac{1}{2} \alpha_{s}^{-2} B_{1,1}+\frac{1}{4} \alpha_{s}^{-2} A_{1} \rho . \\
0=-\frac{1}{2} \alpha_{s}^{-2} B_{1,-1}+\frac{1}{4} \alpha_{s}^{-2} A_{1} \rho .
\end{gathered}
$$

Solving Eq. B.8 for $A_{2}$ we obtain:

$$
A_{2}=\frac{1}{4-\alpha_{s}^{-2}}\left(\frac{A_{1}}{2}\right)=\frac{1}{\left(2 \alpha_{s}\right)^{2}-1}\left(\frac{A_{1}}{2}\right)
$$

Solving Eq. B.7 for the linear $A_{1}$ terms we obtain:

$$
A_{1}=\frac{\alpha_{s}^{-2}}{1-\alpha_{s}^{-2}}\left(-1+\frac{1}{2} A_{2}+\frac{3}{8} A_{1}^{2}\right)
$$

Since $A_{1}$ of the order $\alpha_{s}^{-2}$ (as expected) then the $\alpha_{s}^{-2} A_{2}$ term and the $\alpha_{s}^{-2} A_{1}^{2}$ are of order $\alpha_{s}^{-6}$ and are neglected. Rewriting Eq. B.7 to reflect this, we have:

$$
A_{1}=-\frac{\alpha_{s}^{-2}}{1-\alpha_{s}^{-2}}=-\frac{1}{\alpha_{s}^{2}-1} .
$$

All expressions on the RHS side of Eq. B.12 are of order $\alpha_{s}^{-6}$, therefore we are selfconsistent to exclude the $A_{3}$ term. 
We solve Eq. B.10 and Eq. B.11 for $B_{1, \pm 1}$ and obtain:

$$
\begin{aligned}
B_{1, \pm 1} & =\frac{\alpha_{s}^{-2}}{\left[\alpha_{s}^{-1} \pm 1\right]^{2}-\alpha_{s}^{-2}}\left(\frac{\rho}{2}\right) \\
& =\frac{\alpha_{s}^{-2}}{1 \pm 2 \alpha_{s}^{-1}}\left(\frac{\rho}{2}\right) \\
& =\frac{\alpha_{s}^{-1}}{\alpha_{s} \pm 2}\left(\frac{\rho}{2}\right)
\end{aligned}
$$

where $\sigma$ makes a negligible contribution to $B_{1, \pm 1}$.

All expressions on the RHS of Eq. B.13 and Eq. B.14 are of order $\alpha_{s}^{-4} \rho$, therefore we are self-consistent to exclude the $B_{1,2}$ and $B_{1,-2}$ terms.

For Eq. B.9 we call the $\sigma^{2}$ terms negligible, subtract the bare $\rho$ term from each side and solve for $\sigma$ to obtain the shift in synchrotron frequency:

$$
\sigma=\frac{1}{4}\left(\frac{B_{1,1}+B_{1,-1}}{\rho}+A_{1}\right)
$$

To calculate Eq. B.19 first we must calculate:

$$
\begin{aligned}
\frac{B_{1,1}+B_{1,-1}}{\rho} & =\frac{\alpha_{s}^{-1}}{2}\left(\frac{1}{\alpha_{s}+2}+\frac{1}{\alpha_{s}-2}\right) \\
& =\frac{\alpha_{s}^{-1}}{2}\left(\frac{\alpha_{s}-2+\alpha_{s}+2}{\alpha_{s}^{2}-4}\right) \\
& =\frac{1}{\alpha_{s}^{2}-4} .
\end{aligned}
$$

We substitute Eq. B.20 into Eq. B.19 to obtain:

$$
\begin{aligned}
\sigma & =\frac{1}{4}\left(\frac{1}{\alpha_{s}^{2}-4}-\frac{1}{\alpha_{s}^{2}-1}\right) \\
& =\frac{1}{4}\left(\frac{\alpha_{s}^{2}-1-\alpha_{s}^{2}+4}{\left(\alpha_{s}^{2}-4\right)\left(\alpha_{s}^{2}-1\right)}\right) \\
& =\frac{3}{4} \alpha_{s}^{-4}
\end{aligned}
$$


To summarize, we write these coefficients together:

$$
\begin{aligned}
A_{1} & =-\frac{1}{\alpha_{s}^{2}-1}=-\alpha_{s}^{-2}\left(1+\alpha_{s}^{-2}\right) . \\
A_{2} & =\frac{1}{\left(2 \alpha_{s}\right)^{2}-1}\left(\frac{A_{1}}{2}\right)=-\frac{1}{8} \alpha_{s}^{-4} . \\
B_{1, \pm 1} & =\frac{\alpha_{s}^{-1}}{\alpha_{s} \pm 2}\left(\frac{\rho}{2}\right) . \\
\sigma & =\frac{1}{4}\left(\frac{B_{1,1}+B_{1,-1}}{\rho}+A_{1}\right)=\frac{3}{4} \alpha_{s}^{-4} .
\end{aligned}
$$

\section{DERIVATION OF $\rho$ AND $\psi$ FROM INITIAL CONDITIONS}

At this the synchrotron amplitude $\rho$ and initial synchrotron phase $\psi$ are still undetermined, but we can express them in terms of the initial coordinates. At time $t=0$ (which is fixed to a time in which the relative phase between the $\mathrm{rf}$ cavities is zero), let $\delta=\delta_{0}$ and $\phi=\phi_{0}$.

We have shown that $\phi$ takes the form give in Eq. B.2. We evaluate this expression for $\phi$ at $t=0$, leave the short-hand notation (Eq. B.1), and find $\phi_{0}$ in terms of $\rho$ and $\psi$ :

$$
\phi_{0}=\left(\rho+B_{1,1}+B_{1,-1}\right) \sin (\psi) .
$$

We can calculate $\delta$ from our solution for $\phi$ by taking the derivative:

$$
\begin{aligned}
\delta & =\frac{1}{2 \pi f_{r e v} h \eta} \dot{\phi} . \\
\delta & =\frac{\omega_{\phi}}{2 \pi f_{\text {rev }} h \eta}\left\{A_{1} c_{0,1}+2 A_{2} c_{0,2}+\alpha_{s}^{-1}(1+\sigma) \rho c_{1,0}\right. \\
& \left.+\left[\alpha_{s}^{-1}(1+\sigma)+1\right] B_{1,1} c_{1,1}+\left[\alpha_{s}^{-1}(1+\sigma)-1\right] B_{1,-1} c_{1,-1}\right\} .
\end{aligned}
$$

We evaluate this expression for $\delta$ at $t=0$, leave the short-hand notation (Eq. B.1), and find $\delta_{0}$ in terms of $\rho$ and $\psi$ :

$$
\begin{aligned}
\delta_{0}=\frac{\omega_{\phi}}{2 \pi f_{r e v} h \eta}\{ & A_{1}+2 A_{2} \\
& \left.+\left[\left(\frac{1+\sigma}{\alpha_{s}}\right)\left(\rho+B_{1,1}+B_{1,-1}\right)+\alpha_{s}\left(B_{1,1}-B_{1,-1}\right)\right] \cos (\psi)\right\} .
\end{aligned}
$$


Next we solve Eq. B.26 and Eq. B.29 for $\Phi_{0}=\rho \sin (\psi)$ and $\Delta_{0}=\rho \cos (\psi)$ :

$$
\begin{gathered}
\Phi_{0}=\left(1+\frac{B_{1,1}+B_{1,-1}}{\rho}\right)^{-1} \phi_{0} . \\
\Delta_{0}=\alpha_{s}\left[1+\frac{B_{1,1}+B_{1,-1}}{\rho}+\alpha_{s} \frac{B_{1,1}-B_{1,-1}}{\rho}+\sigma\left(1+\frac{B_{1,1}+B_{1,-1}}{\rho}\right)\right]^{-1} \\
\times\left(\frac{2 \pi f_{r e v} h \eta}{\omega_{\phi}} \delta_{0}-A_{1}-2 A_{2}\right) .
\end{gathered}
$$

$\phi_{0}$ and $\delta_{0}$ have been translated by the initial position of the bucket center and rescaled to obtain the expressions for $\Phi_{0}$ and $\Delta_{0}$. Using $\Phi_{0}=\rho \sin (\psi)$ and $\Delta_{0}=\rho \cos (\psi)$, the solution for $\rho$ and $\psi$ be written as:

$$
\begin{aligned}
\rho & =\sqrt{\Phi_{0}^{2}+\Delta_{0}^{2}} . \\
\psi & =\operatorname{sgn}\left(\Phi_{0}\right) \arccos \left(\frac{\Delta_{0}}{\rho}\right) .
\end{aligned}
$$

Eq. B.30 and Eq. B.31 can be further simplified by writing the $B_{1,1}$ and $B_{1,-1}$ terms explicitly in terms of $\alpha_{s}$. We calculate:

$$
\begin{aligned}
\alpha_{s} \frac{B_{1,1}-B_{1,-1}}{\rho} & =\frac{1}{2}\left(\frac{1}{\alpha_{s}+2}-\frac{1}{\alpha_{s}-2}\right) \\
& =\frac{1}{2}\left(\frac{\alpha_{s}-2-\alpha_{s}-2}{\alpha_{s}^{2}-4}\right) \\
& =\frac{-2}{\alpha_{s}^{2}-4} .
\end{aligned}
$$

We apply Eq. B.20 and Eq. B.34 to Eq. B.30 and Eq. B.31 to obtain:

$$
\begin{aligned}
\Phi_{0}= & \left(1+\frac{1}{\alpha_{s}^{2}-4}\right)^{-1} \phi_{0}=\frac{\alpha_{s}^{2}-4}{\alpha_{s}^{2}-3} \phi_{0} . \\
\Delta_{0}= & \alpha_{s}\left[1+\frac{1}{\alpha_{s}^{2}-4}-\frac{2}{\alpha_{s}^{2}-4}+\sigma\left(1+\frac{1}{\alpha_{s}^{2}-4}\right)\right]^{-1} \\
& \times\left(\frac{2 \pi f_{r e v} h \eta}{\omega_{\phi}} \delta_{0}-A_{1}-2 A_{2}\right) . \\
\Delta_{0}= & \frac{\alpha_{s}\left(\alpha_{s}^{2}-4\right)}{\left(\alpha_{s}^{2}-5\right)+\sigma \alpha_{s}^{2}}\left(\frac{2 \pi f_{r e v} h \eta}{\omega_{\phi}} \delta_{0}-A_{1}-2 A_{2}\right) .
\end{aligned}
$$


$\Phi_{0}$ and $\Delta_{0}$ are fully expanded as follows:

$$
\begin{aligned}
& \Phi_{0}=\phi_{0}\left(1-\alpha_{s}^{-2}-3 \alpha_{s}^{-4}\right) . \\
& \Delta_{0}=\alpha_{s}\left(\frac{2 \pi f_{r e v} h \eta}{\omega_{\phi}} \delta_{0}+\alpha_{s}^{-2}+\frac{5}{4} \alpha_{s}^{-4}\right)\left(1+\alpha_{s}^{-2}+\frac{17}{4} \alpha_{s}^{-4}\right) .
\end{aligned}
$$

Eq. B.38 and Eq. B.39 can then be substituted into Eq. B.32 and Eq. B.33 to obtain $\rho$ and $\psi$ respectively. Then $\rho$ can be substituted into Eq. B.24 to obtain $B_{1,1}$ and $B_{1,-1}$. 


\section{STABILITY MAPS FOR SLIP-STACKING}

This appendix shows the stability maps of the slip-stacking phase-space for values of $\alpha_{s}$ from 2.3 to 8.0 in descending 0.1 increments. Recall that stability map is a visualization of a numerical integration of the following equation:

$$
\dot{\phi}=2 \pi f_{r e v} h \eta \delta, \quad \dot{\delta}=f_{r e v} V_{\delta}\left[\sin (\phi)+\sin \left(\phi-\omega_{\phi} t\right)\right] .
$$

Where $\phi$ is the phase of the particle relative to the RF cavity and $\delta$ is the fractional deviation from a reference momentum. Some stability maps are particularly noteworthy. Recall that $\alpha_{s}=6.2$ maximizes the phase-space area and $\alpha_{s}=5.5$ maximizes the momentum acceptance.
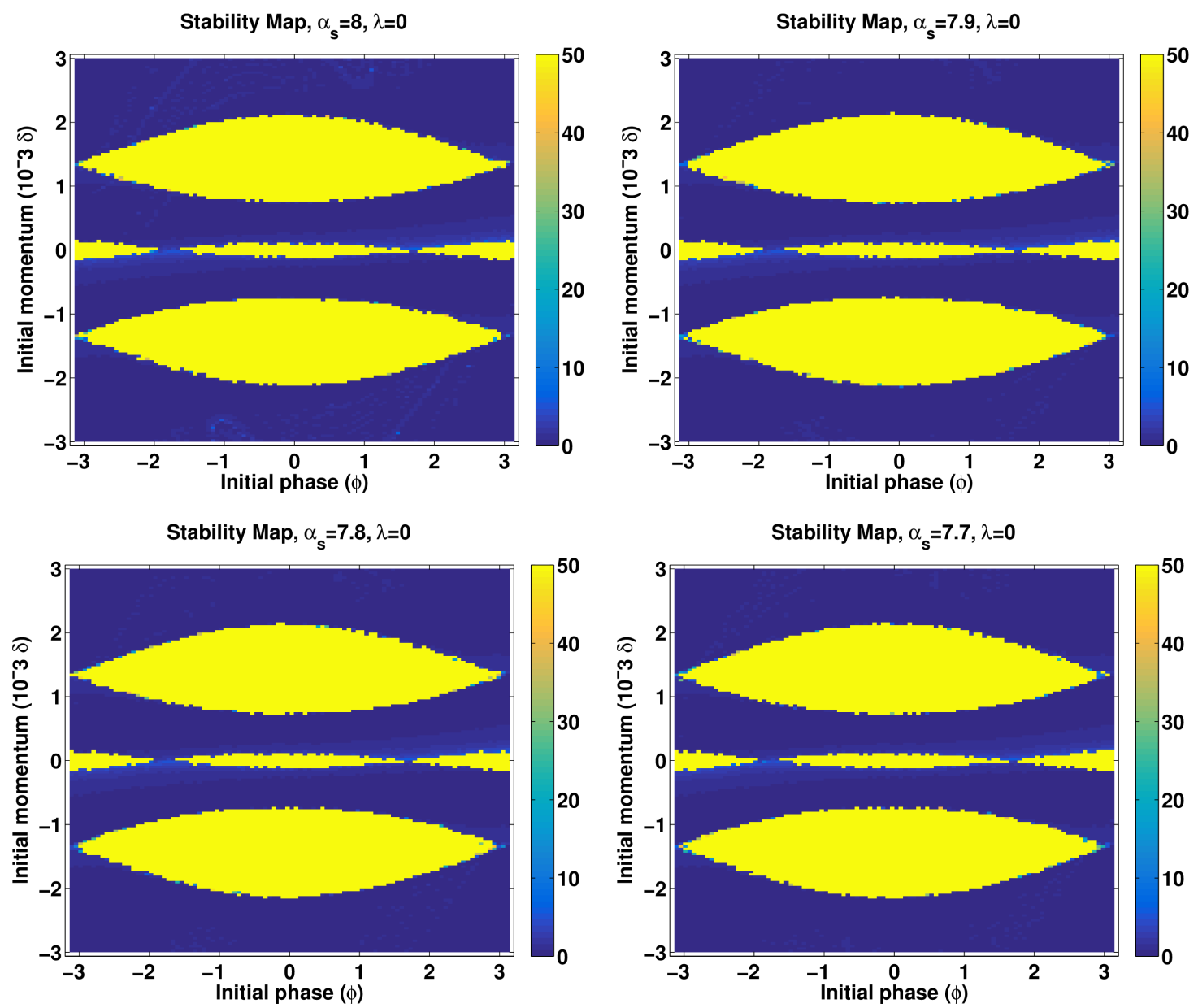

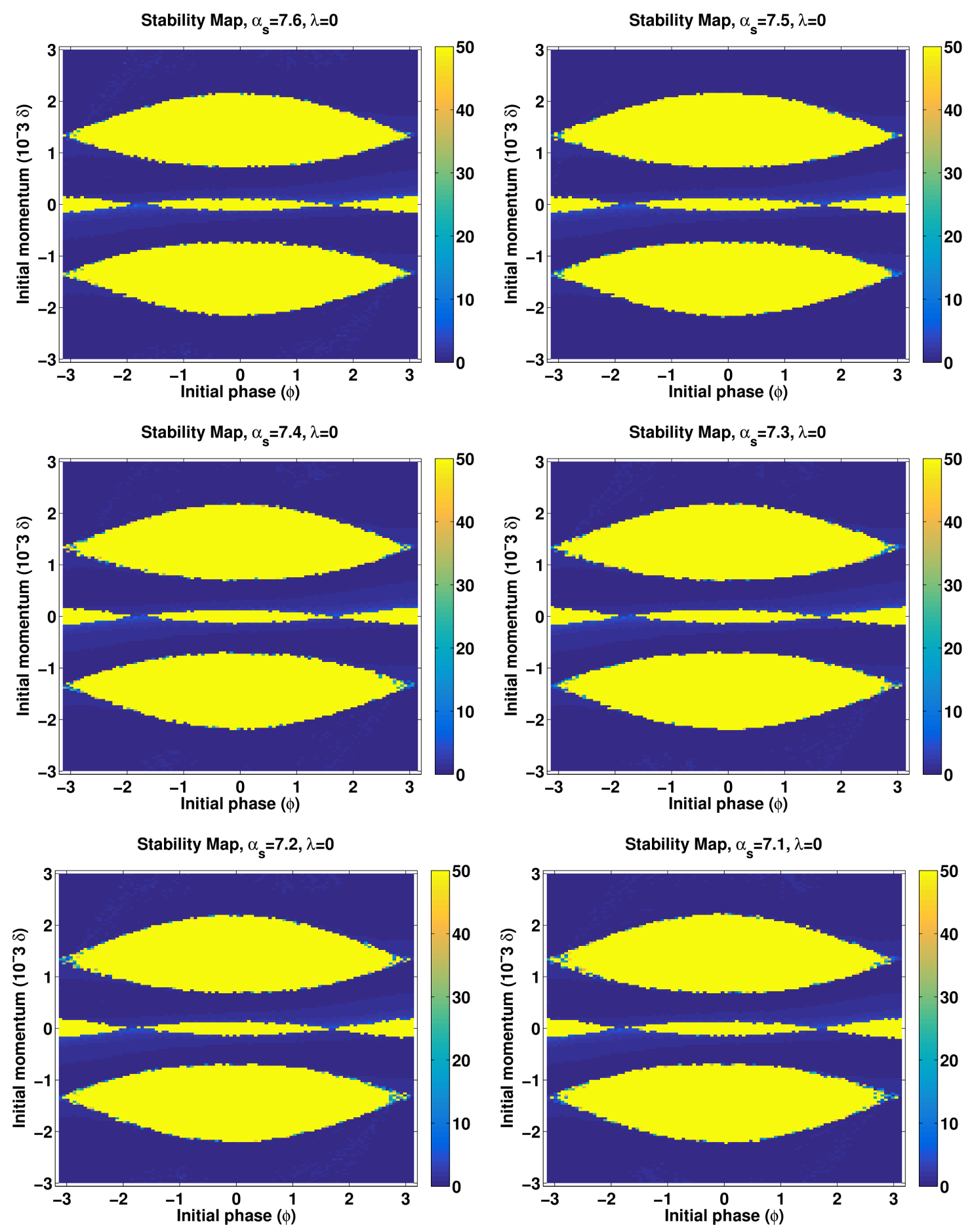
Stability Map, $\alpha_{s}=7, \lambda=0$

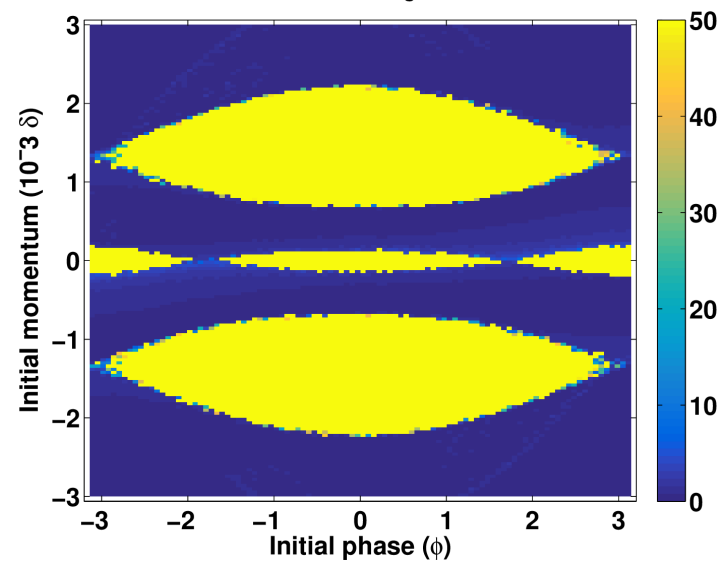

Stability Map, $\alpha_{s}=6.8, \lambda=0$

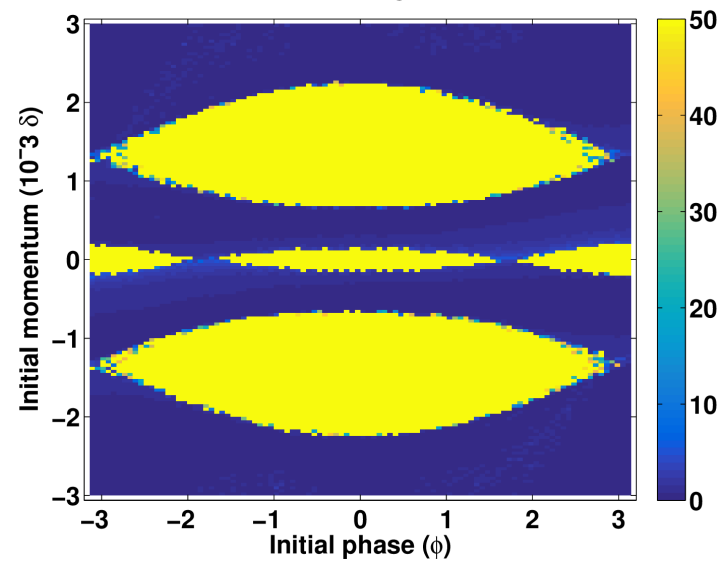

Stability Map, $\alpha_{s}=6.6, \lambda=0$

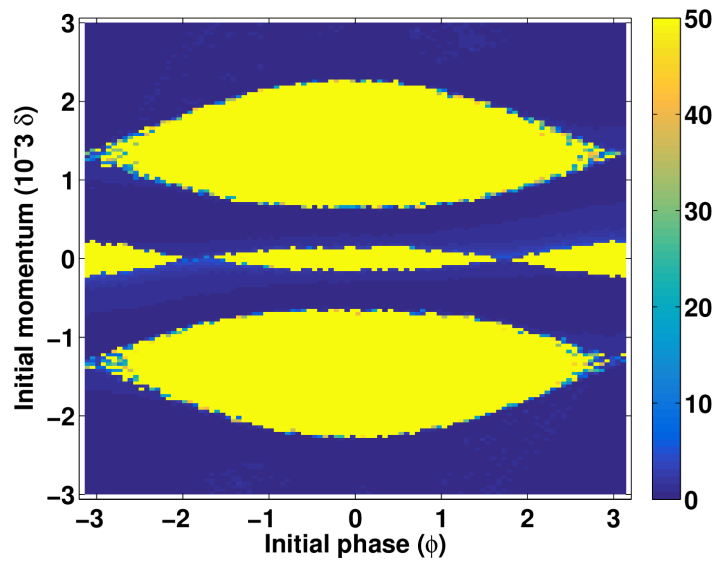

Stability Map, $\alpha_{s}=6.9, \lambda=0$

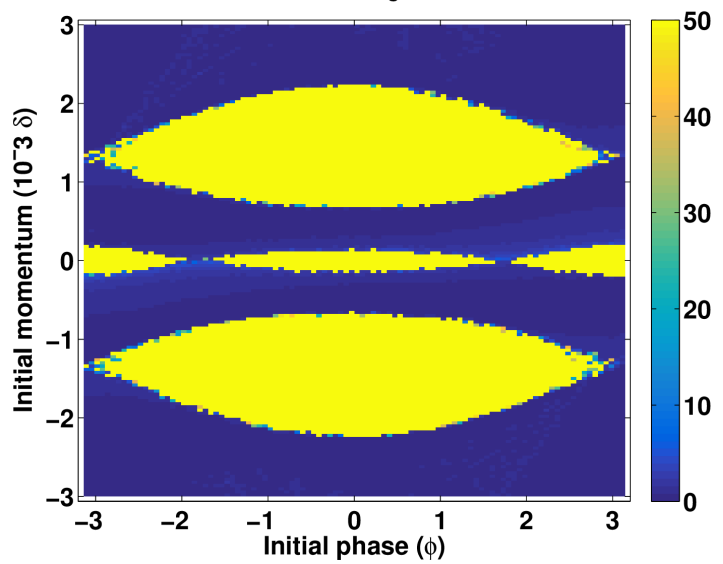

Stability Map, $\alpha_{s}=6.7, \lambda=0$

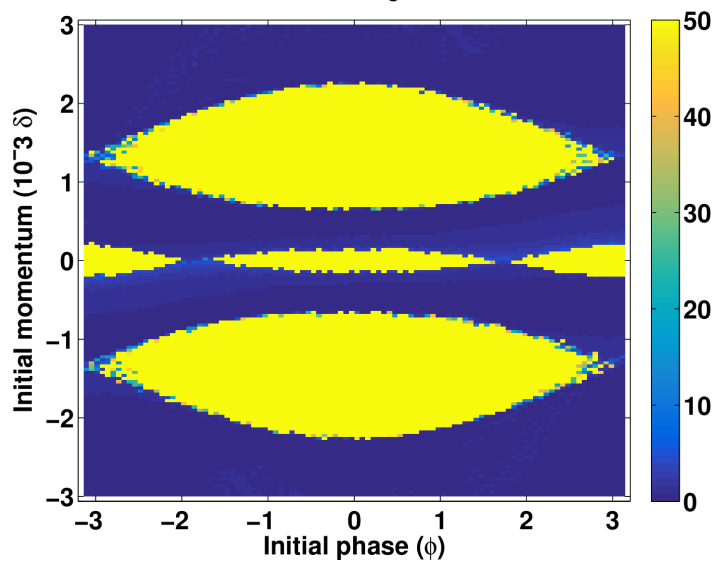

Stability Map, $\alpha_{s}=6.5, \lambda=0$

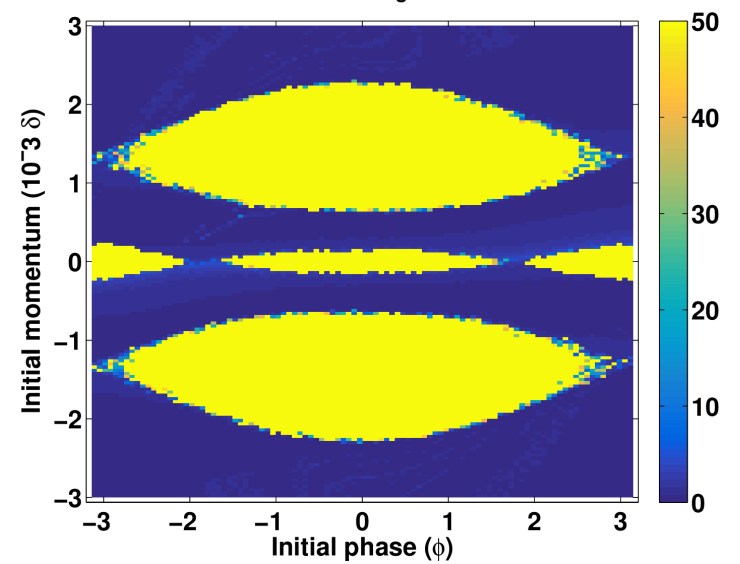



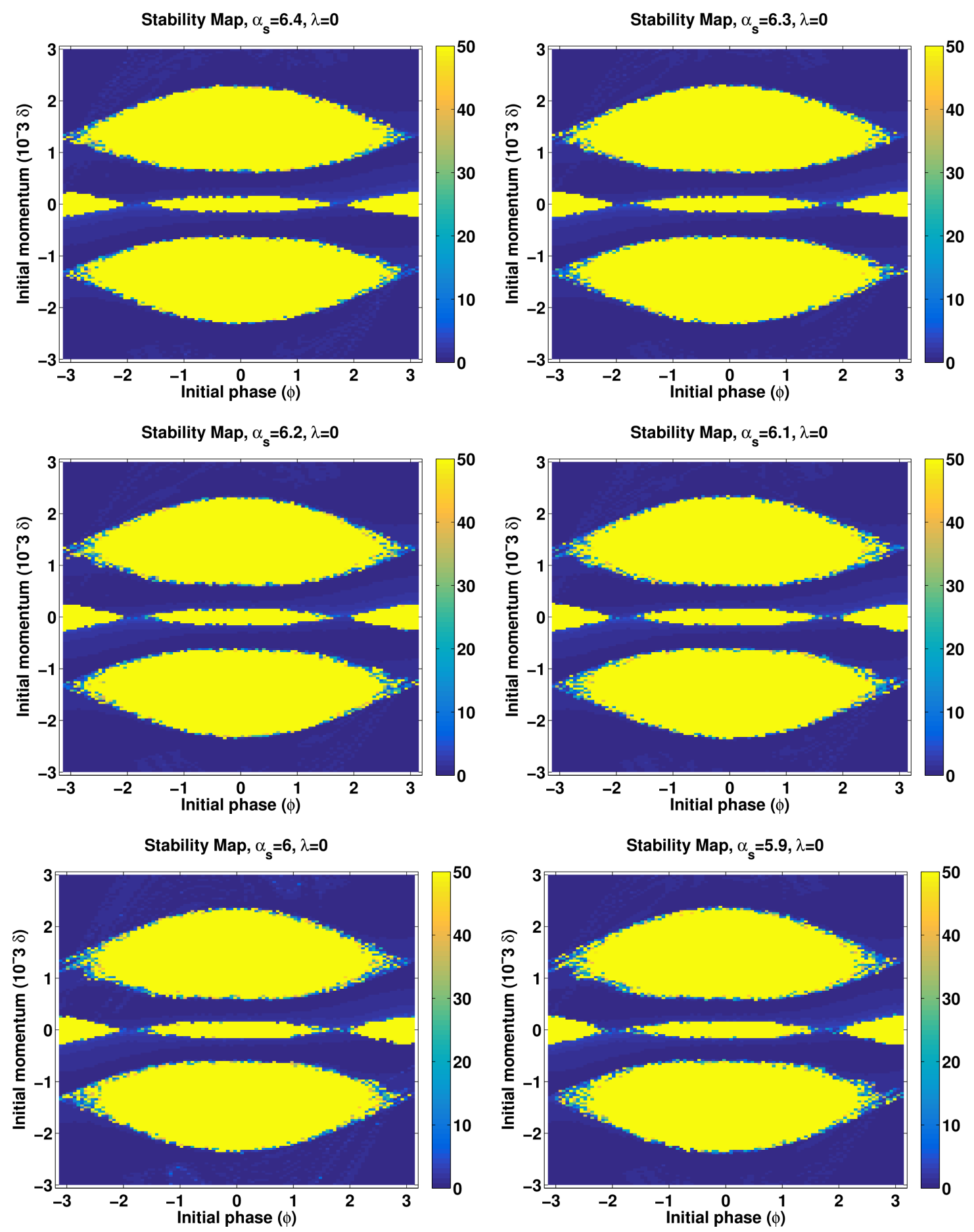

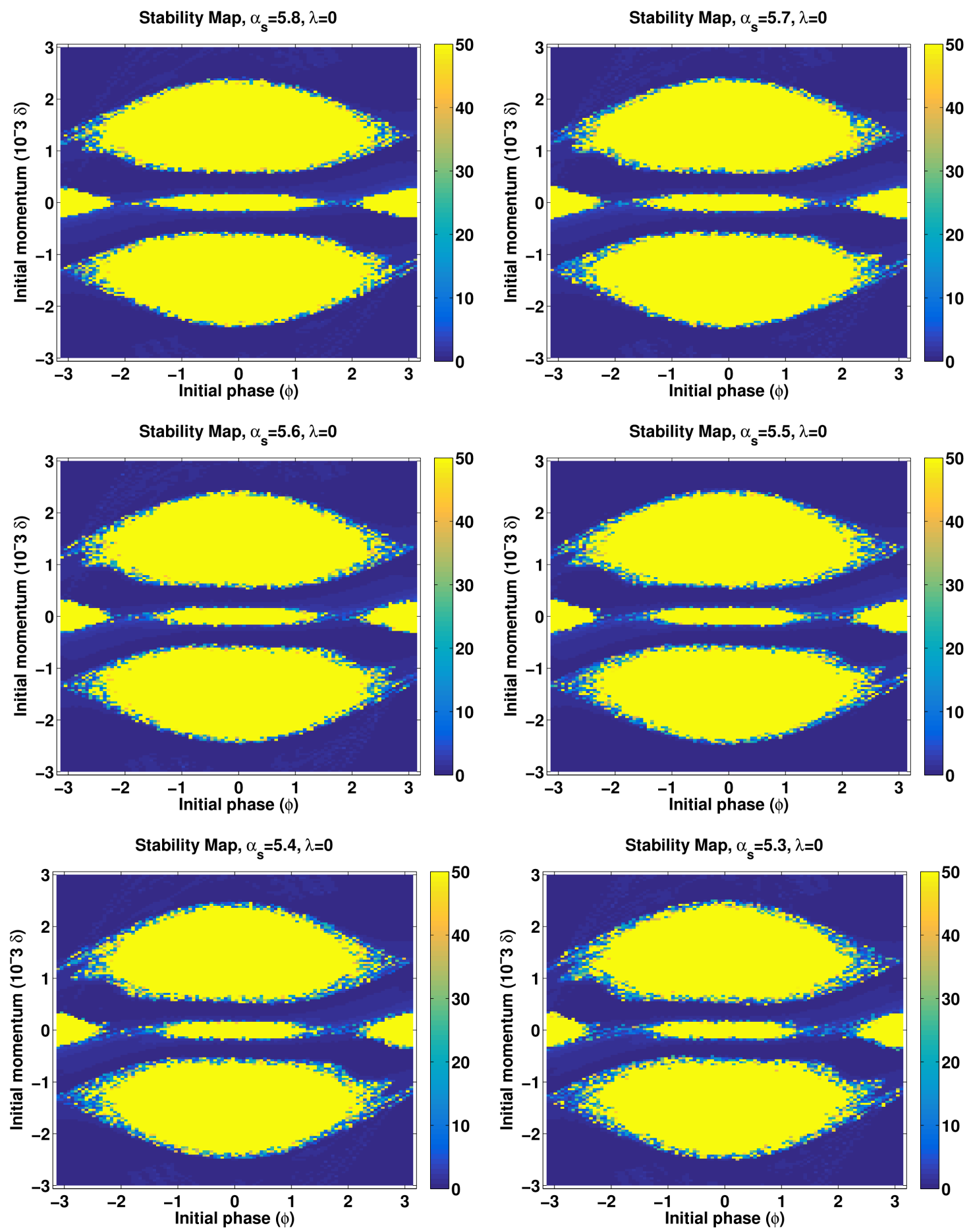

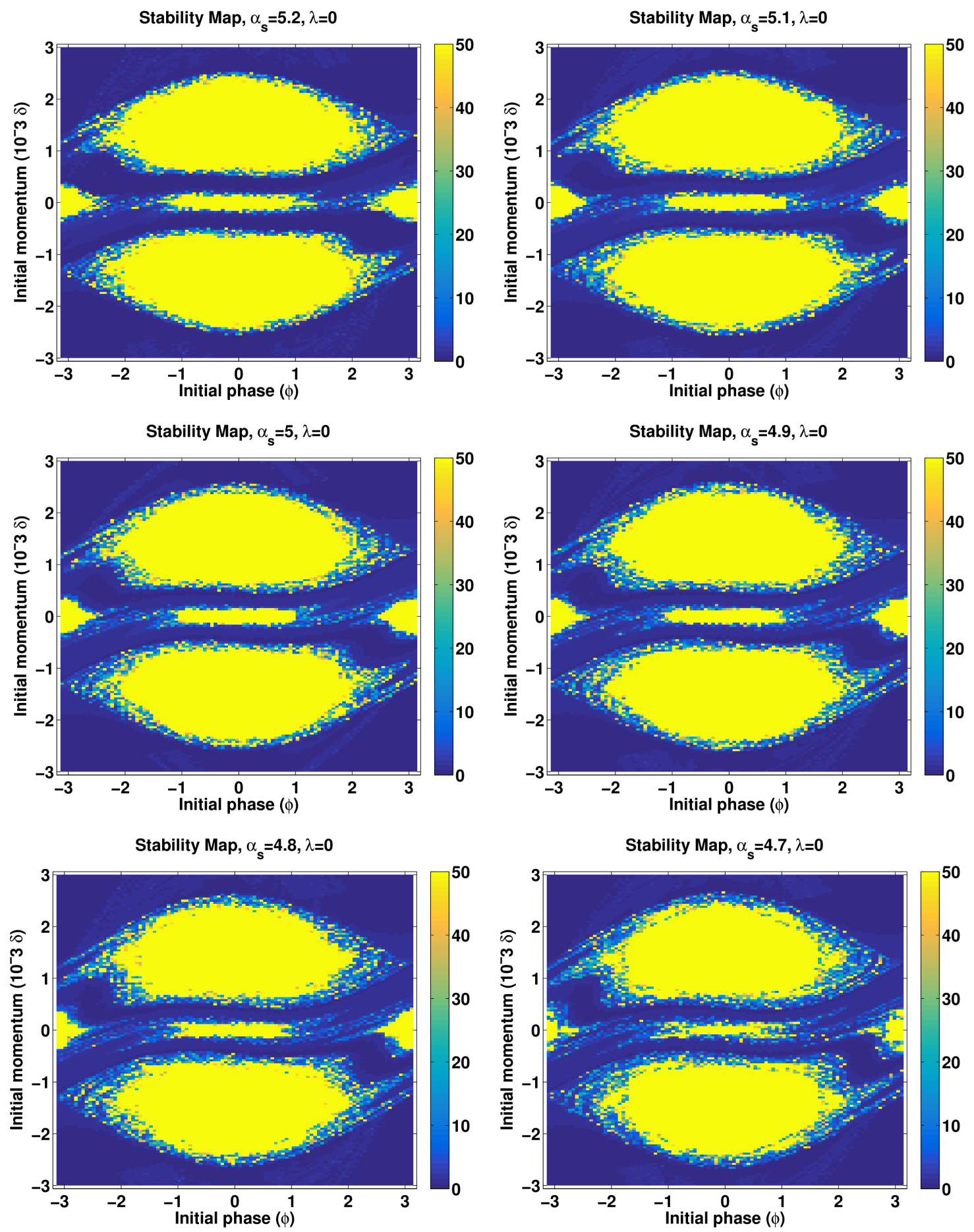

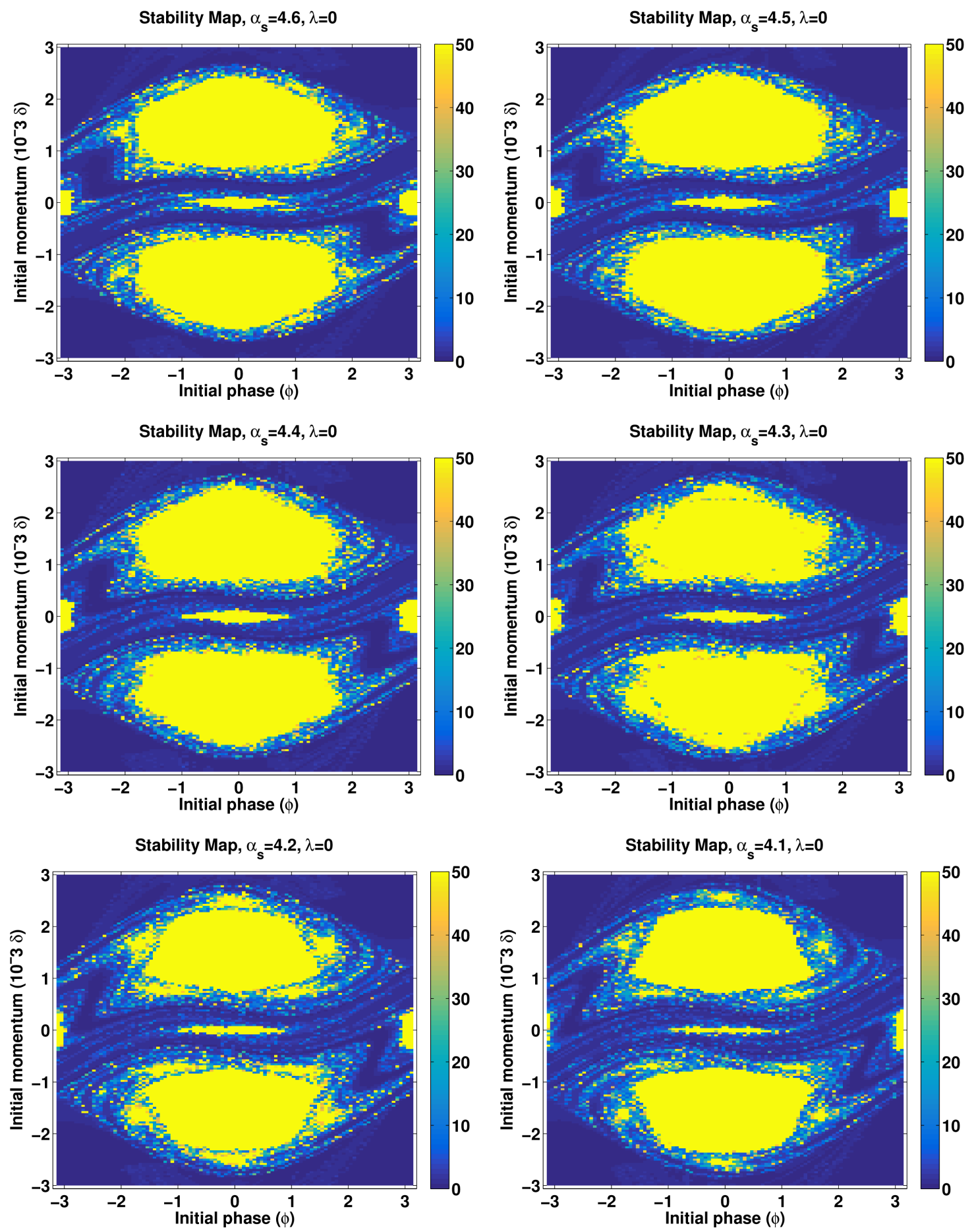

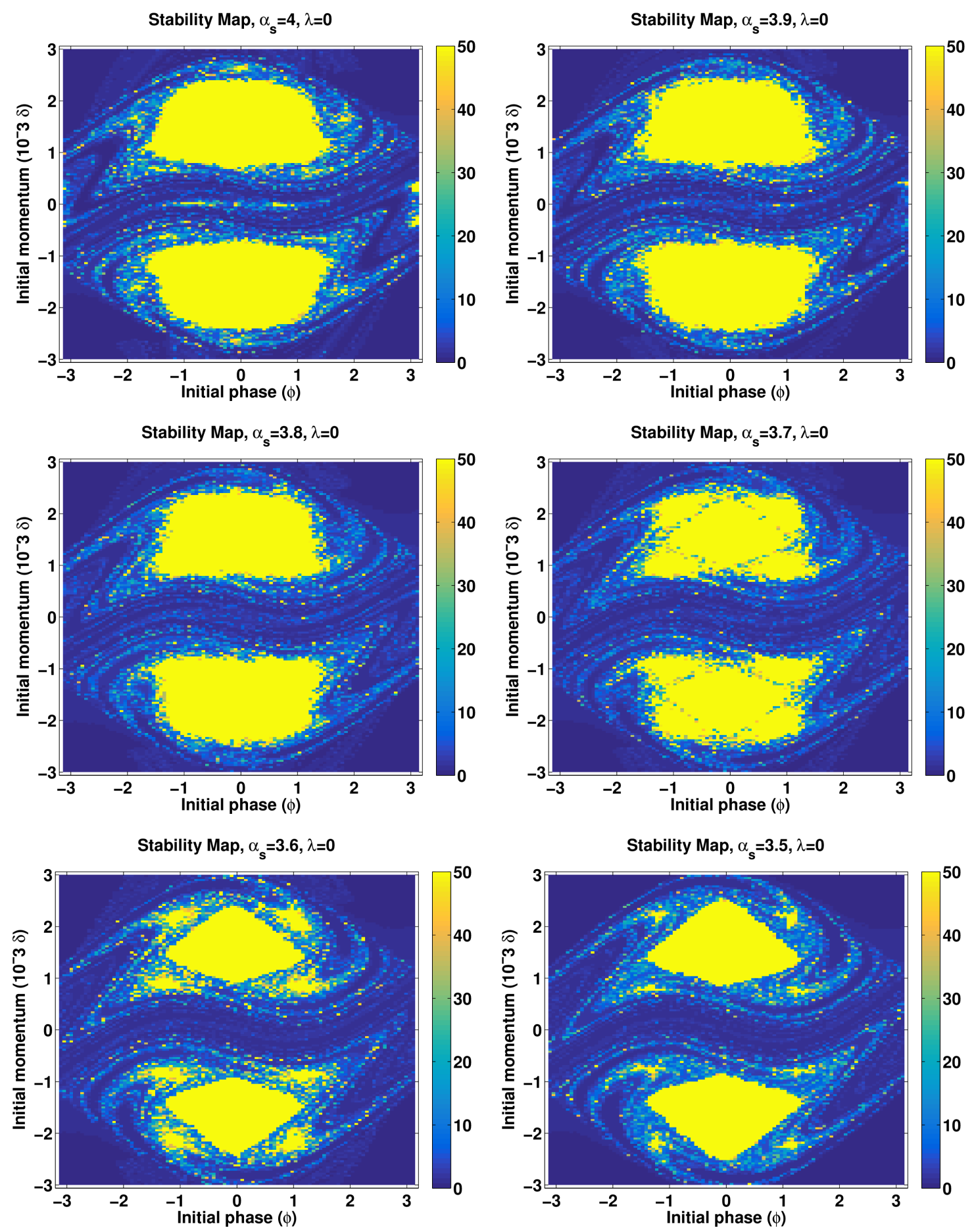

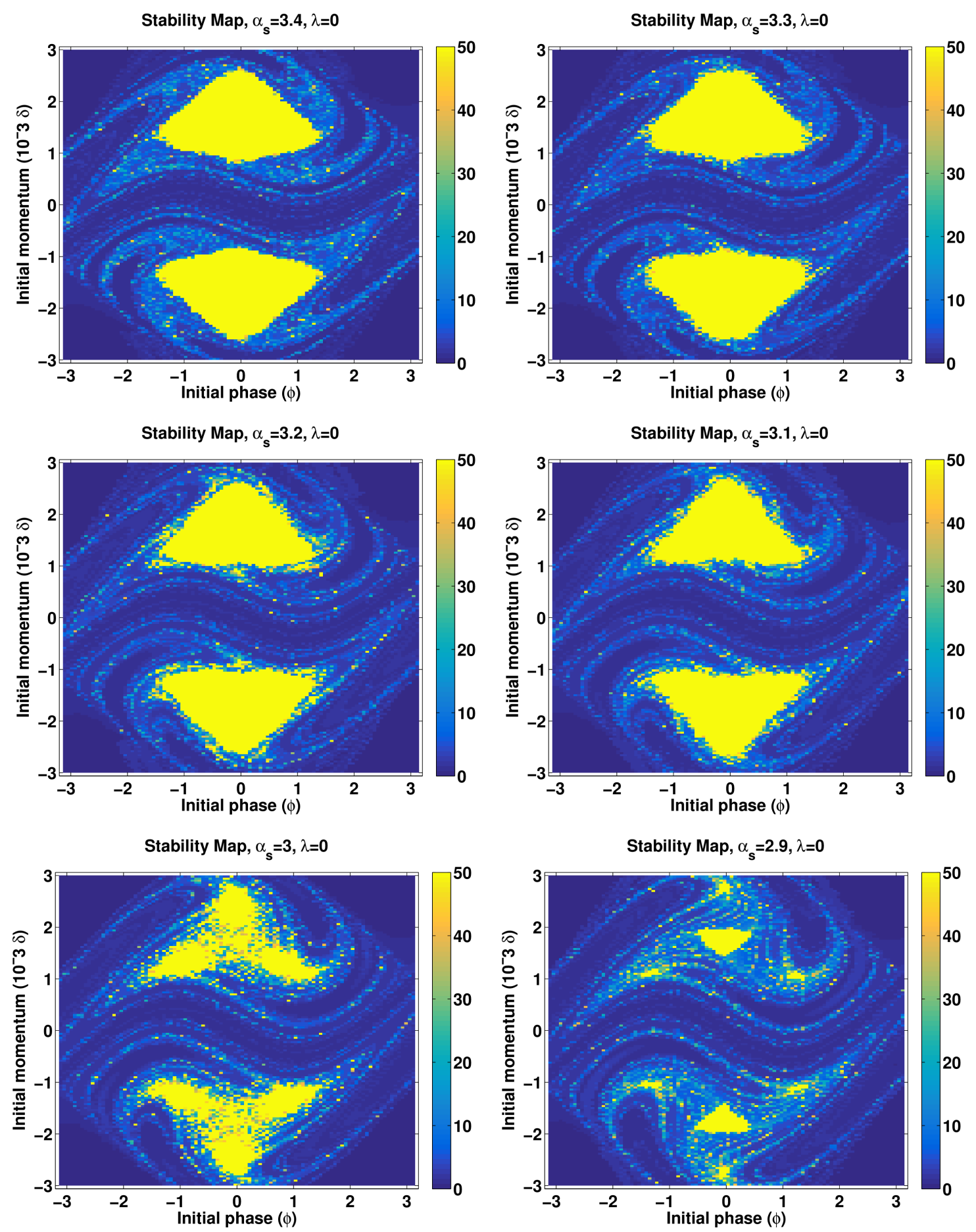

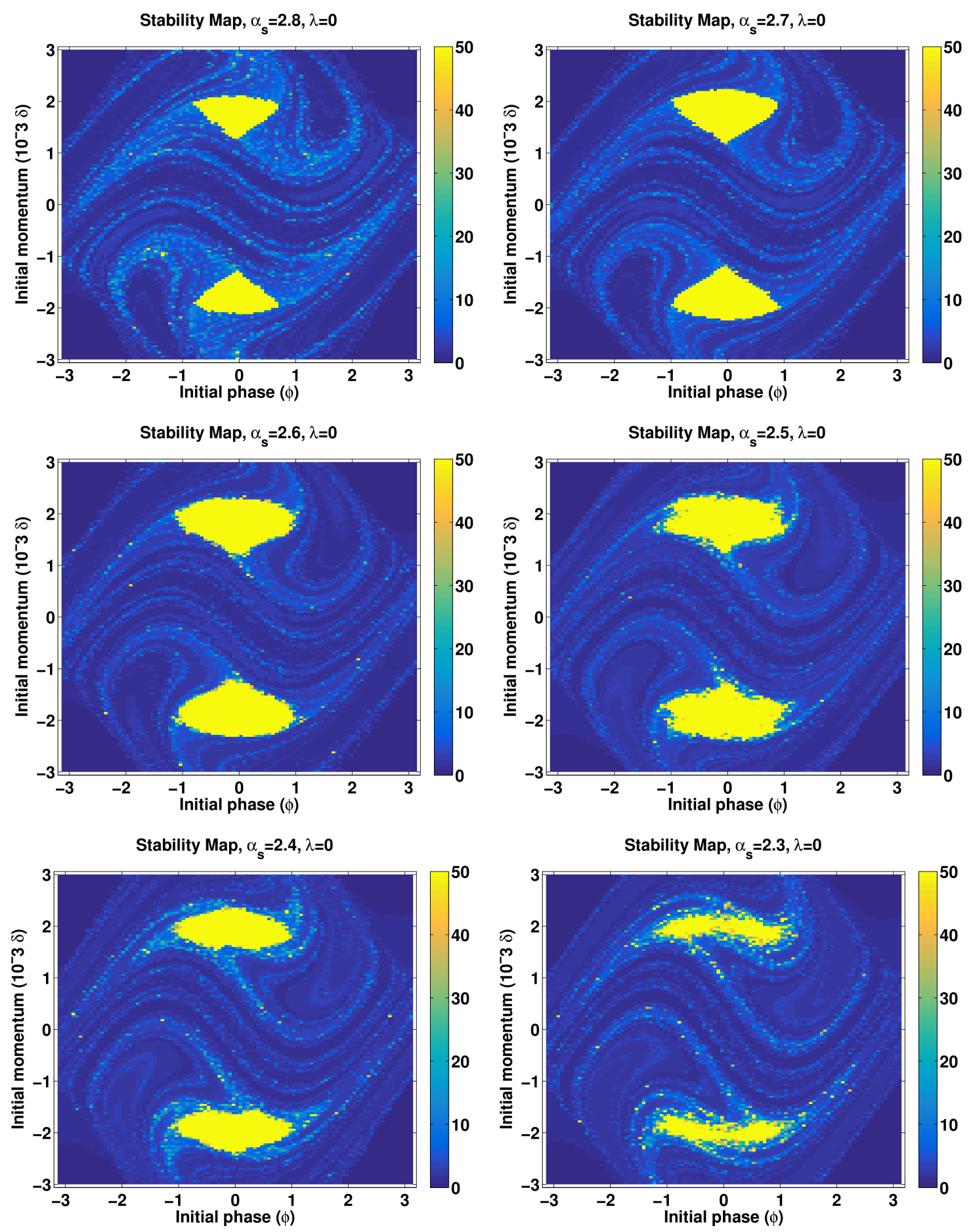


\section{STABILITY MAPS FOR HARMONIC SLIP-STACKING}

This appendix shows the stability maps of the slip-stacking phase-space for values of $\alpha_{s}$ from 2.5 to 8.0 in descending 0.1 increments and $\lambda$ optimized for the stable phase-space area. Recall that stability map is a visualization of a numerical integration of the following equation:

$$
\dot{\phi}=2 \pi f_{r e v} h \eta \delta, \dot{\delta}=f_{r e v} V_{\delta}\left[\sin (\phi)+\sin \left(\phi-\omega_{\phi} t\right)+\lambda \sin \left(2 \phi-\omega_{\phi} t\right)\right] .
$$

Where $\phi$ is the phase of the particle relative to the RF cavity and $\delta$ is the fractional deviation from a reference momentum.
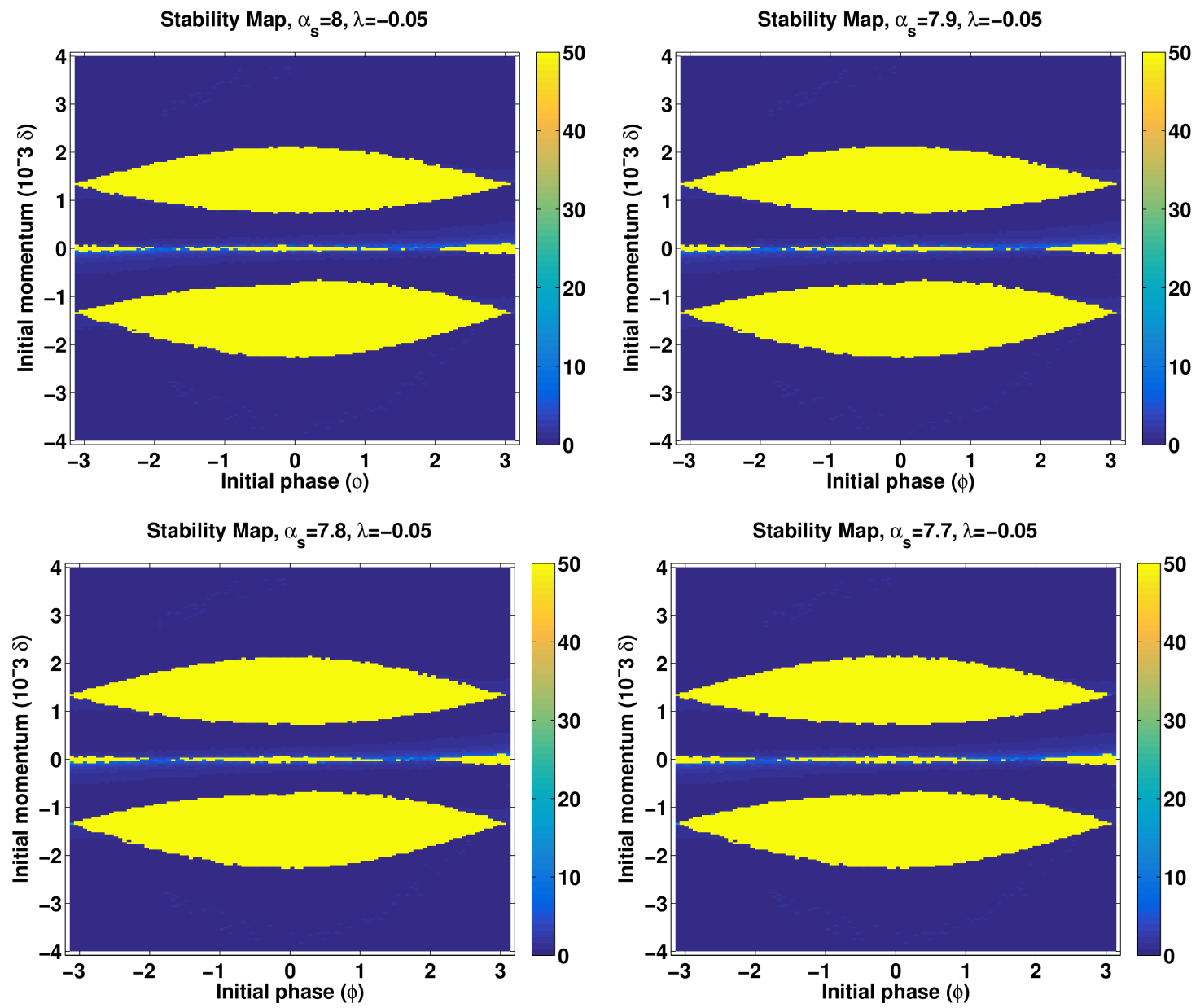

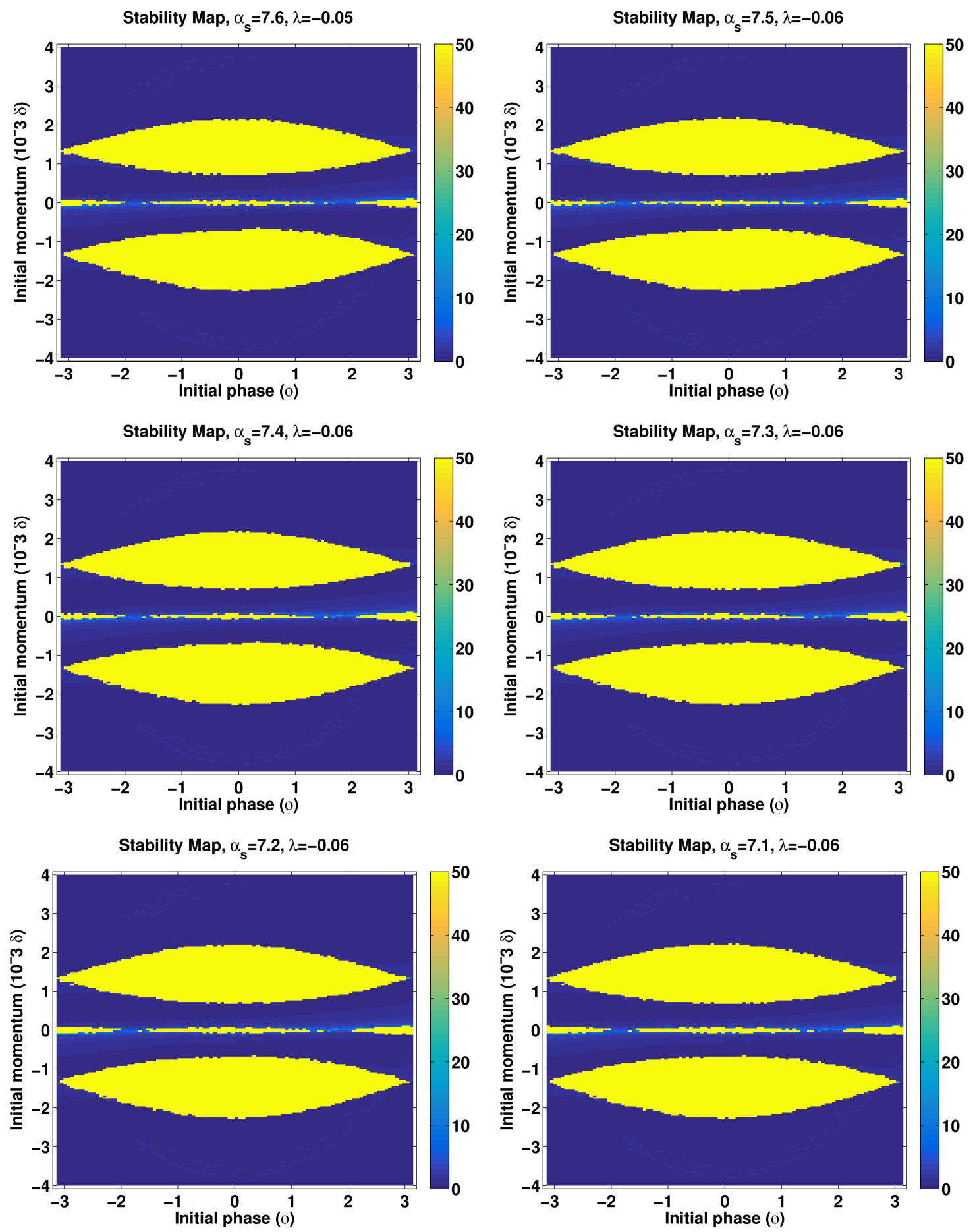
Stability Map, $\alpha_{s}=7, \lambda=-0.06$

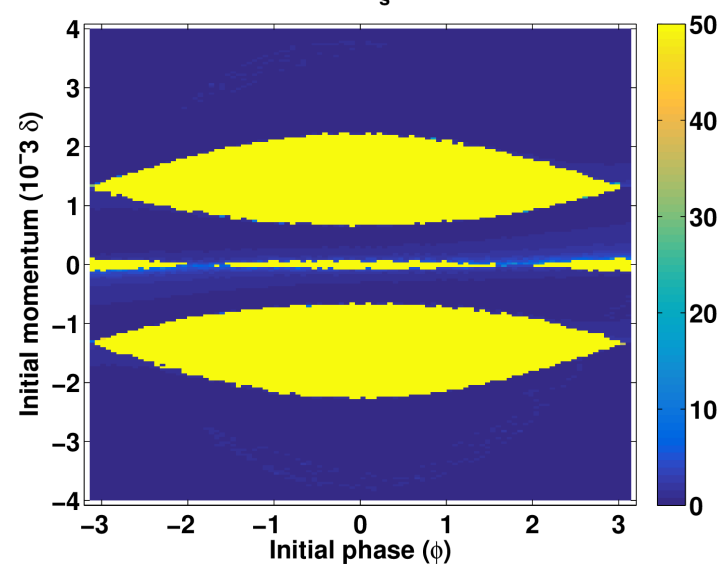

Stability Map, $\alpha_{s}=6.8, \lambda=-0.07$

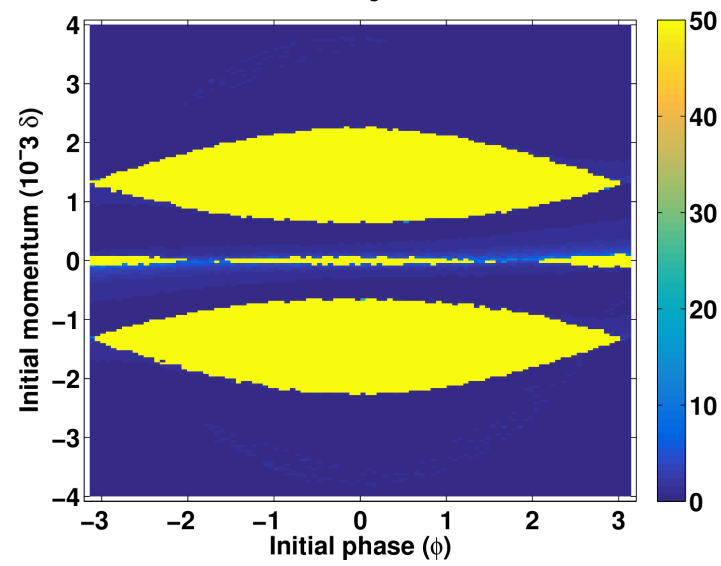

Stability Map, $\alpha_{s}=6.6, \lambda=-0.07$

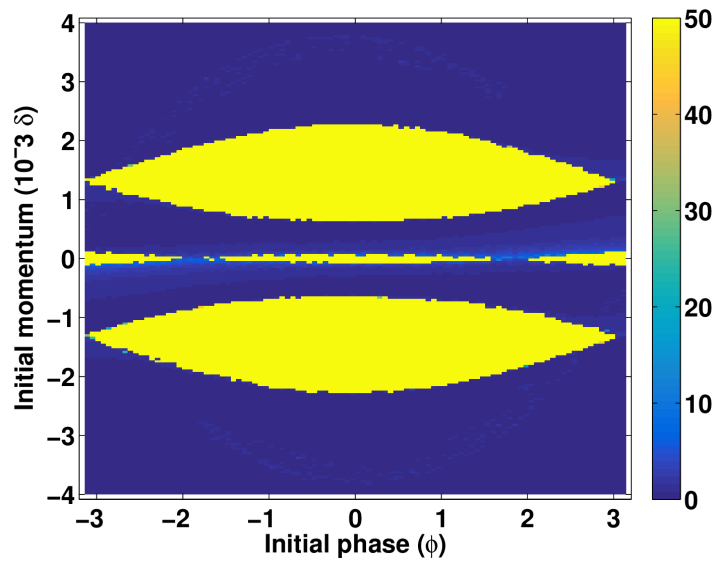

Stability Map, $\alpha_{s}=6.9, \lambda=-0.07$

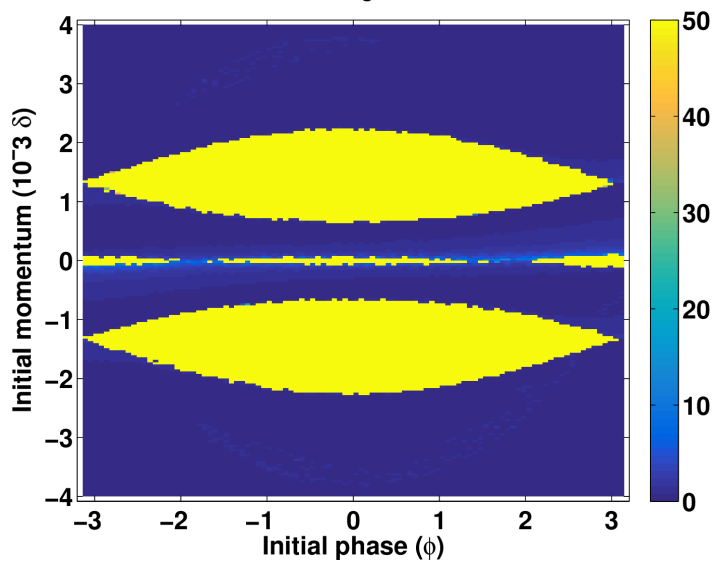

Stability Map, $\alpha_{s}=6.7, \lambda=-0.07$

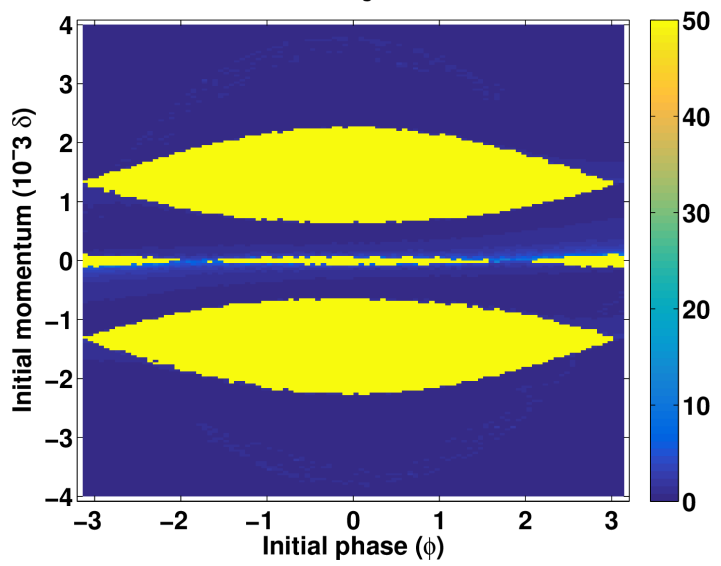

Stability Map, $\alpha_{s}=6.5, \lambda=-0.08$

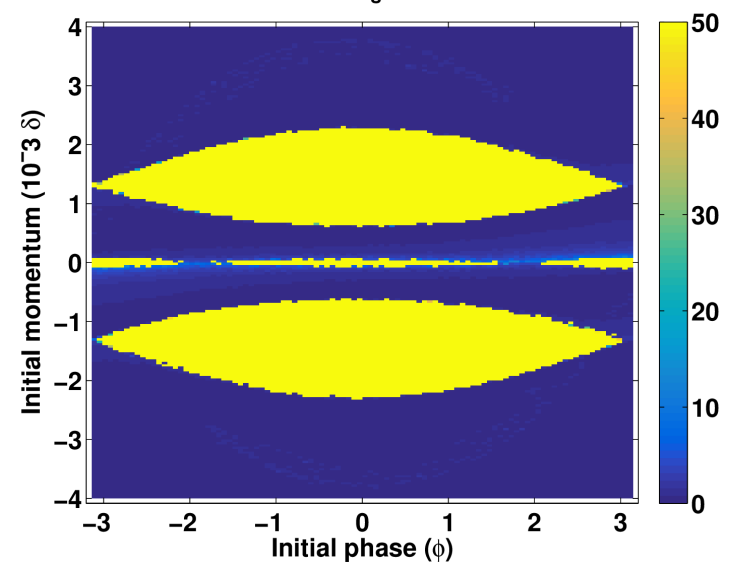



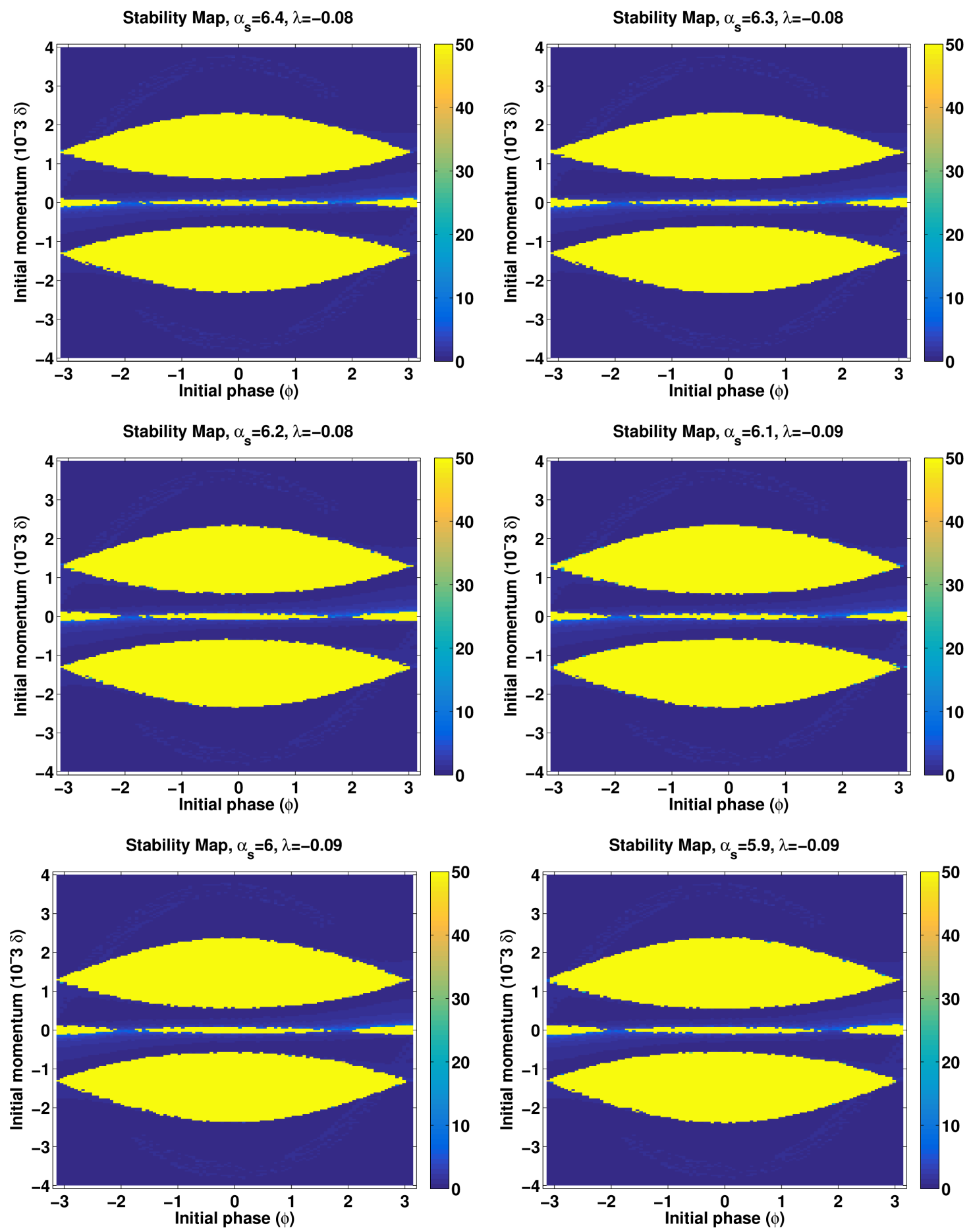

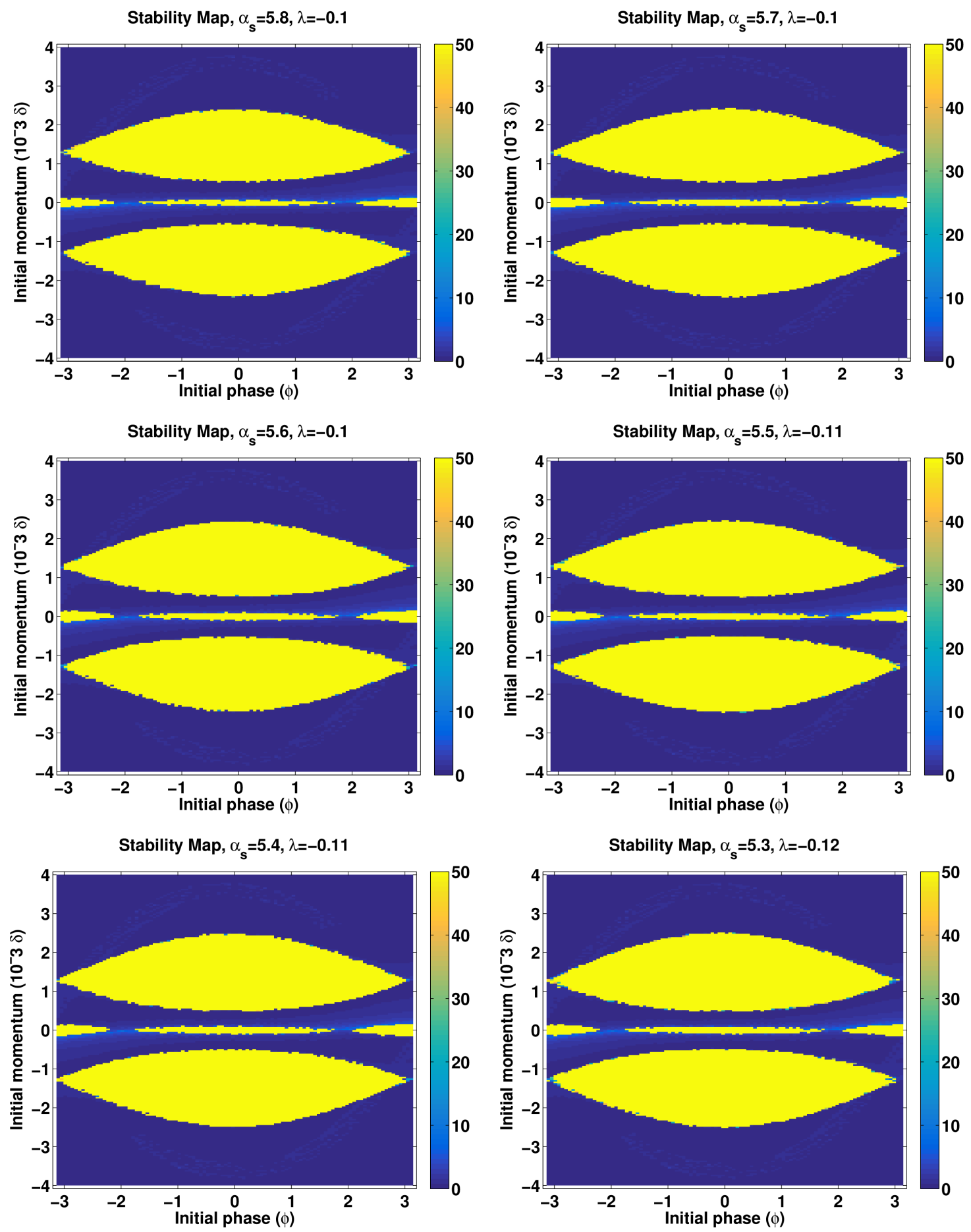

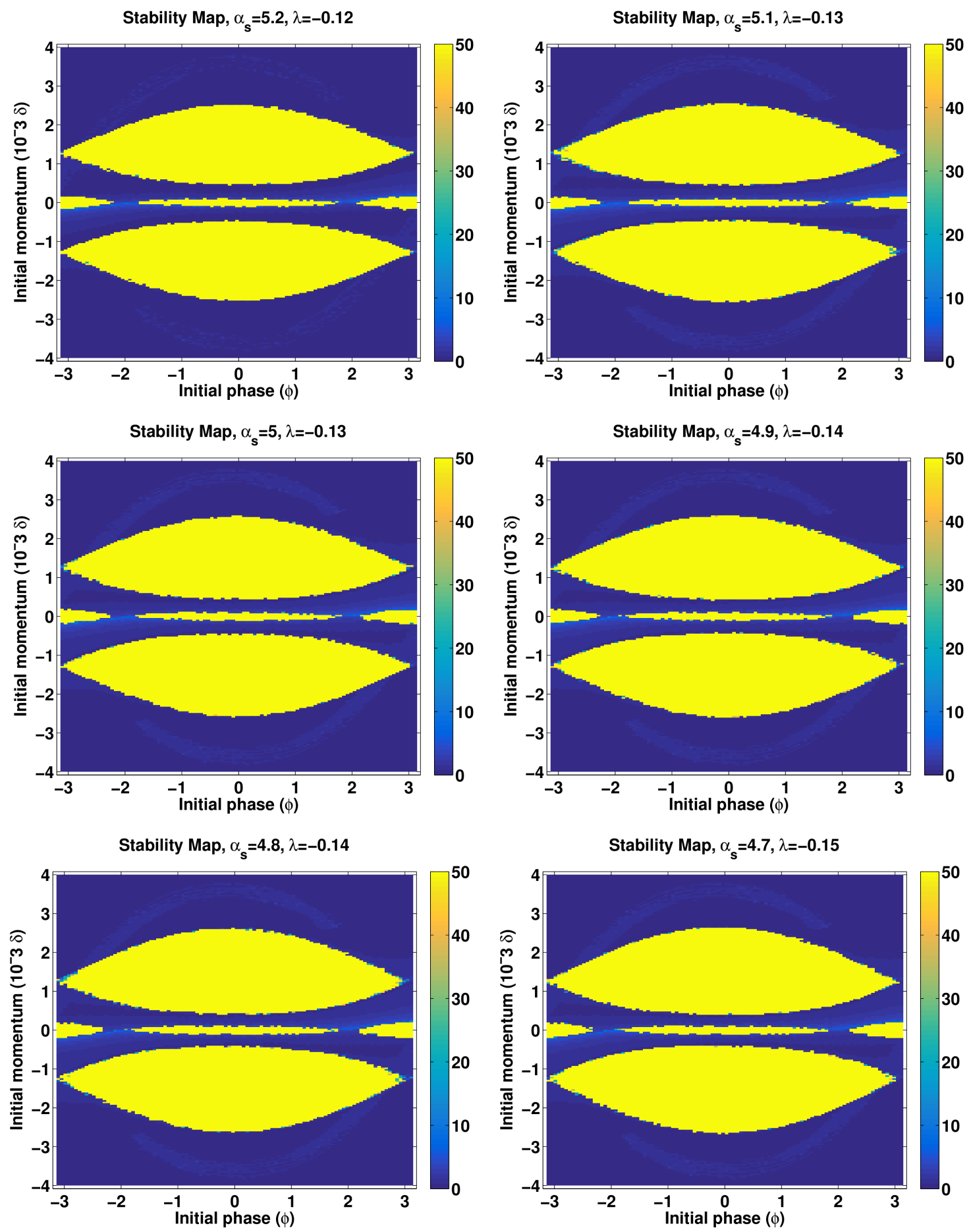

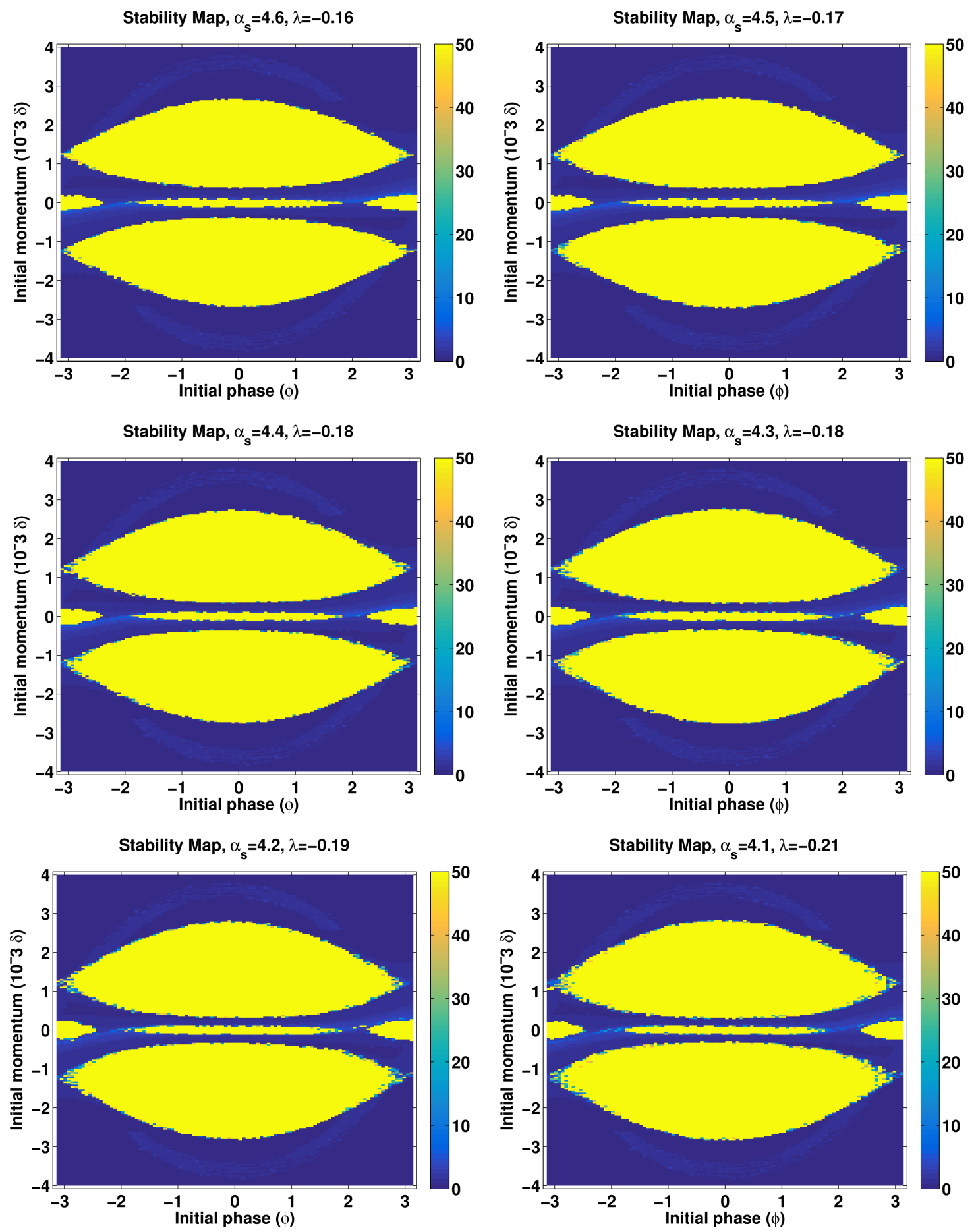
Stability Map, $\alpha_{s}=4, \lambda=-0.22$

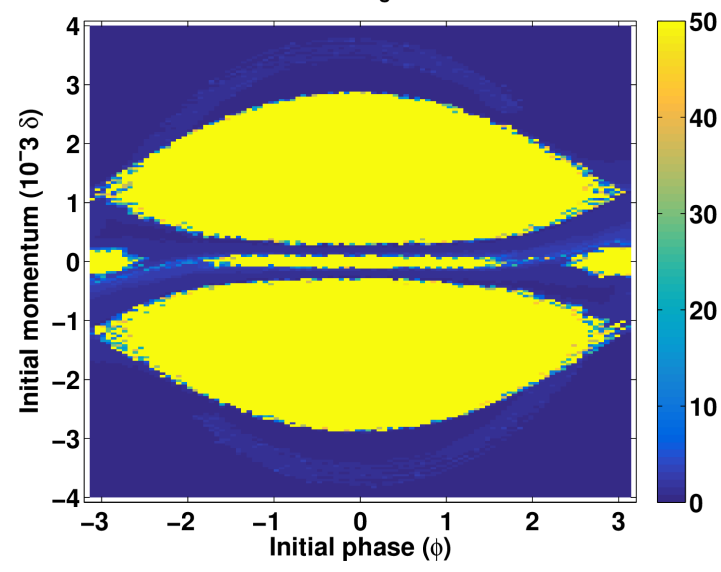

Stability Map, $\alpha_{s}=3.8, \lambda=-0.25$

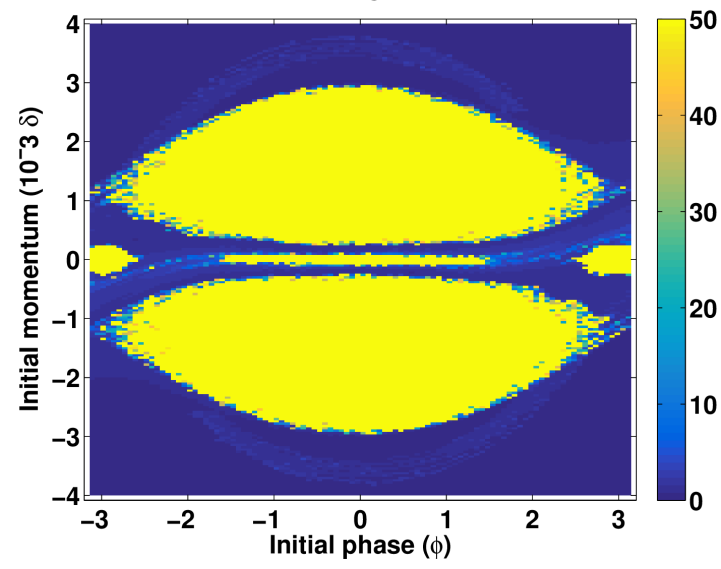

Stability Map, $\alpha_{s}=3.6, \lambda=-0.27$

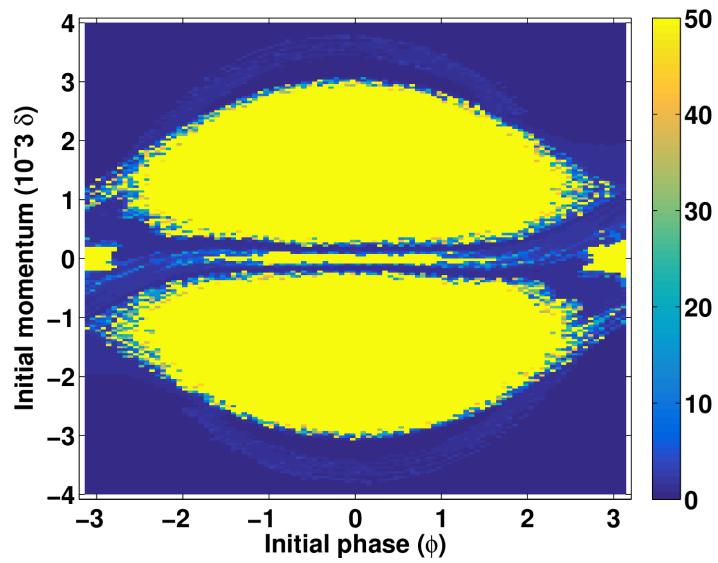

Stability Map, $\alpha_{s}=3.9, \lambda=-0.23$

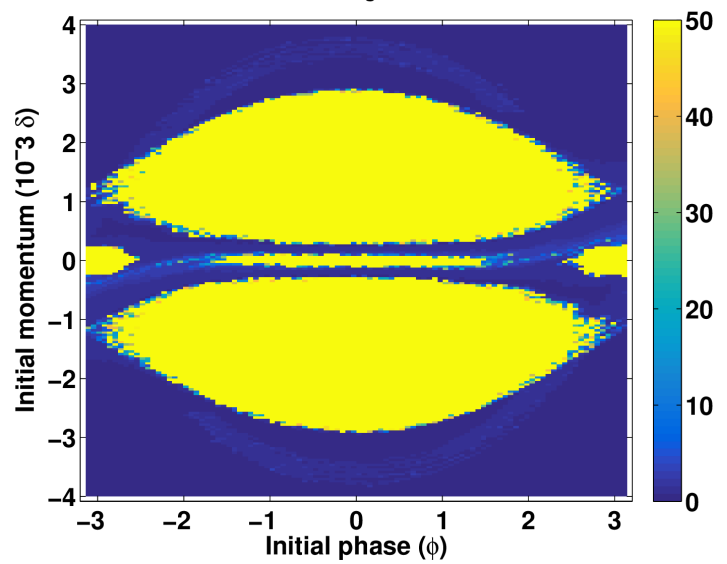

Stability Map, $\alpha_{s}=3.7, \lambda=-0.26$

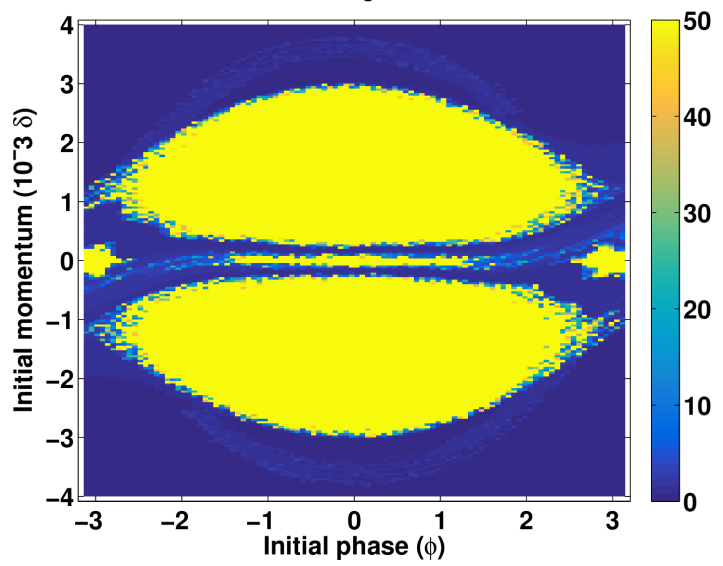

Stability Map, $\alpha_{s}=3.5, \lambda=-0.29$

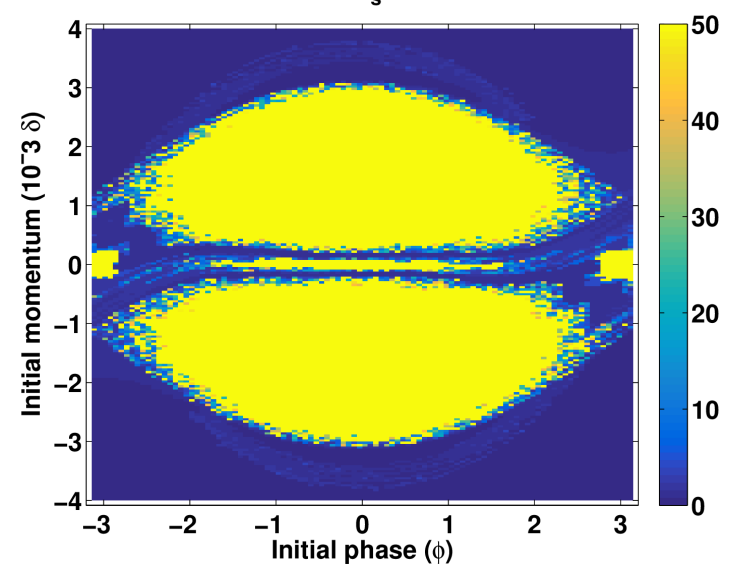



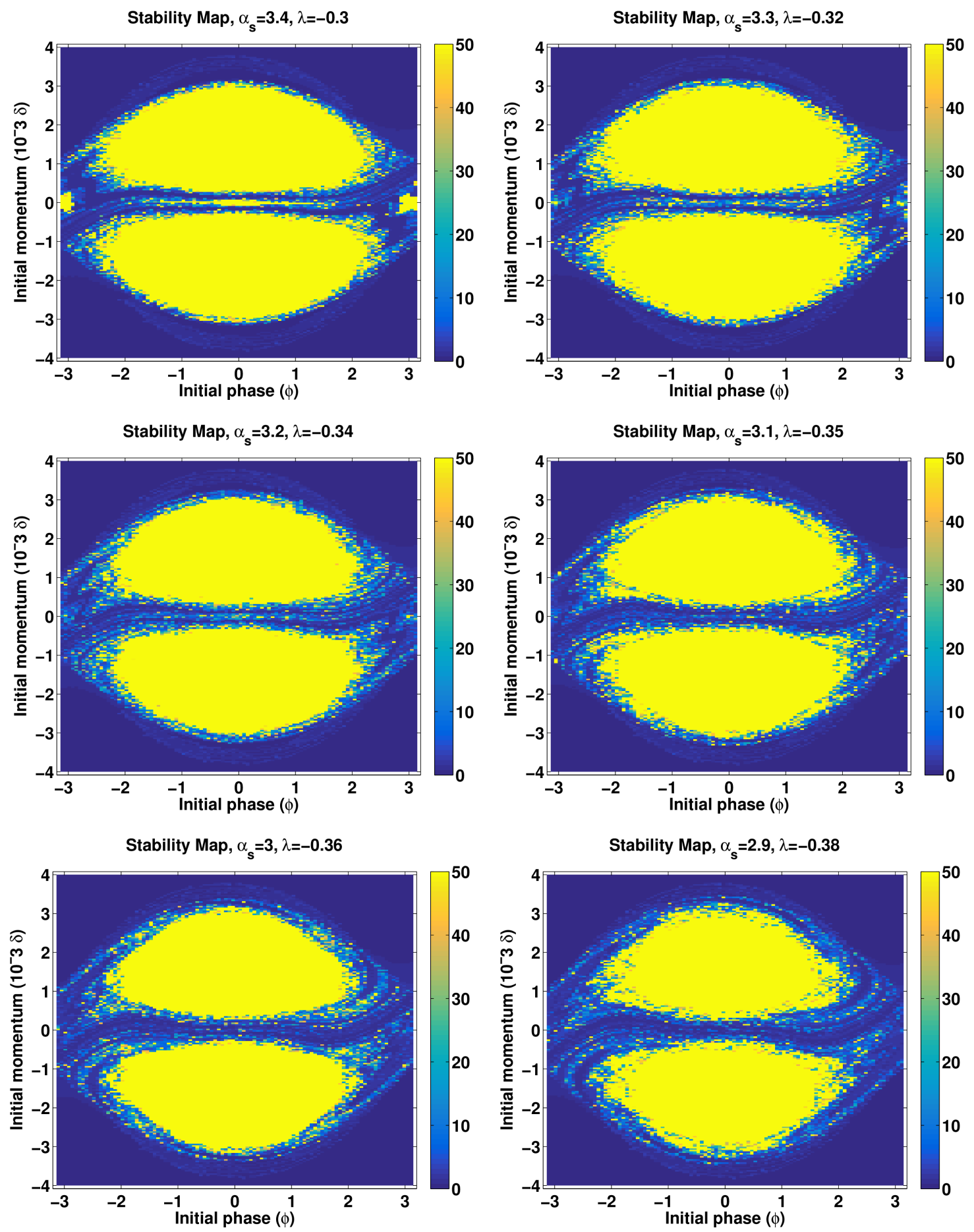

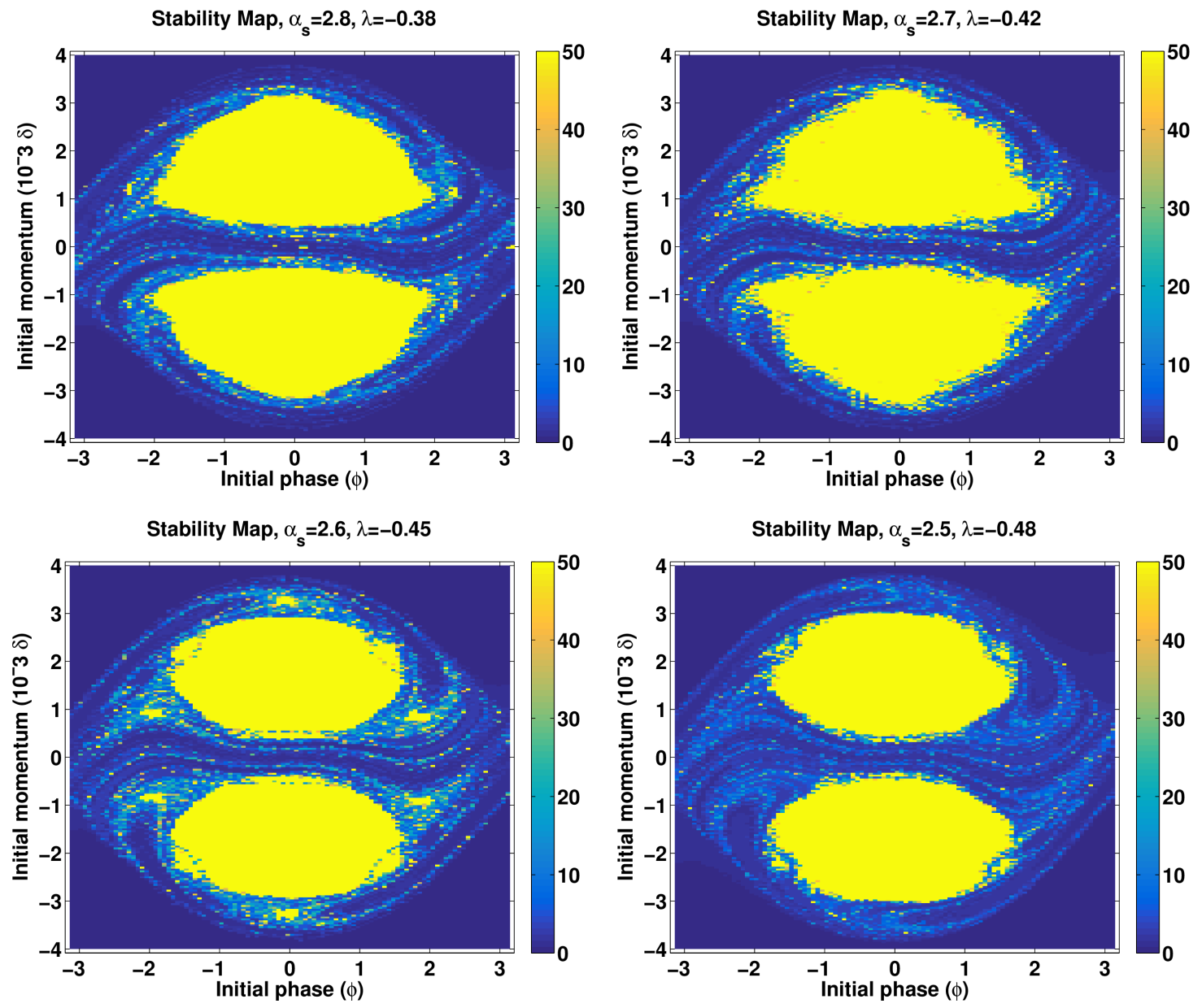


\section{E POINCARÉ MAPS FOR HARMONIC SLIP-STACKING}

This appendix shows the stability maps of the higher slip-stacking bucket for values of $\alpha_{s}$ from 3 to 8 in ascending 1 increments and $\lambda$ in increments of 0.01 around the optimal value. Recall that the Poincaré maps show the coordinates of a phase-space trajectories at $2 \pi / \omega_{\phi}$ intervals. The following differential equation is used:

$$
\dot{\phi}=2 \pi f_{\text {rev }} h \eta \delta, \dot{\delta}=f_{\text {rev }} V_{\delta}\left[\sin (\phi)+\sin \left(\phi-\omega_{\phi} t\right)+\lambda \sin \left(2 \phi-\omega_{\phi} t\right)\right]
$$

Where $\phi$ is the phase of the particle relative to the $\mathrm{RF}$ cavity and $\delta$ is the fractional deviation from a reference momentum.

POINCARÉ MAPS, $\alpha_{s}=3$
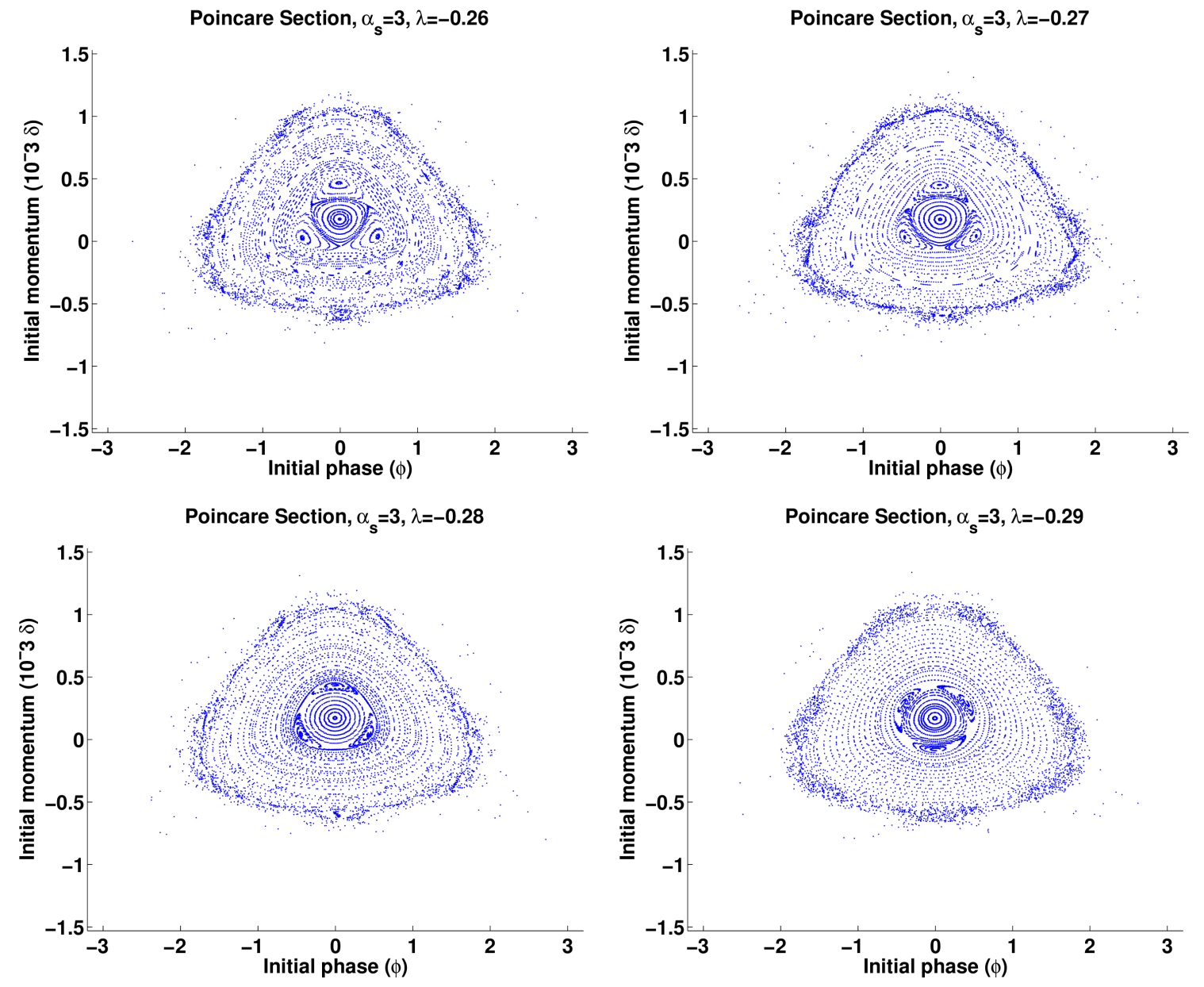

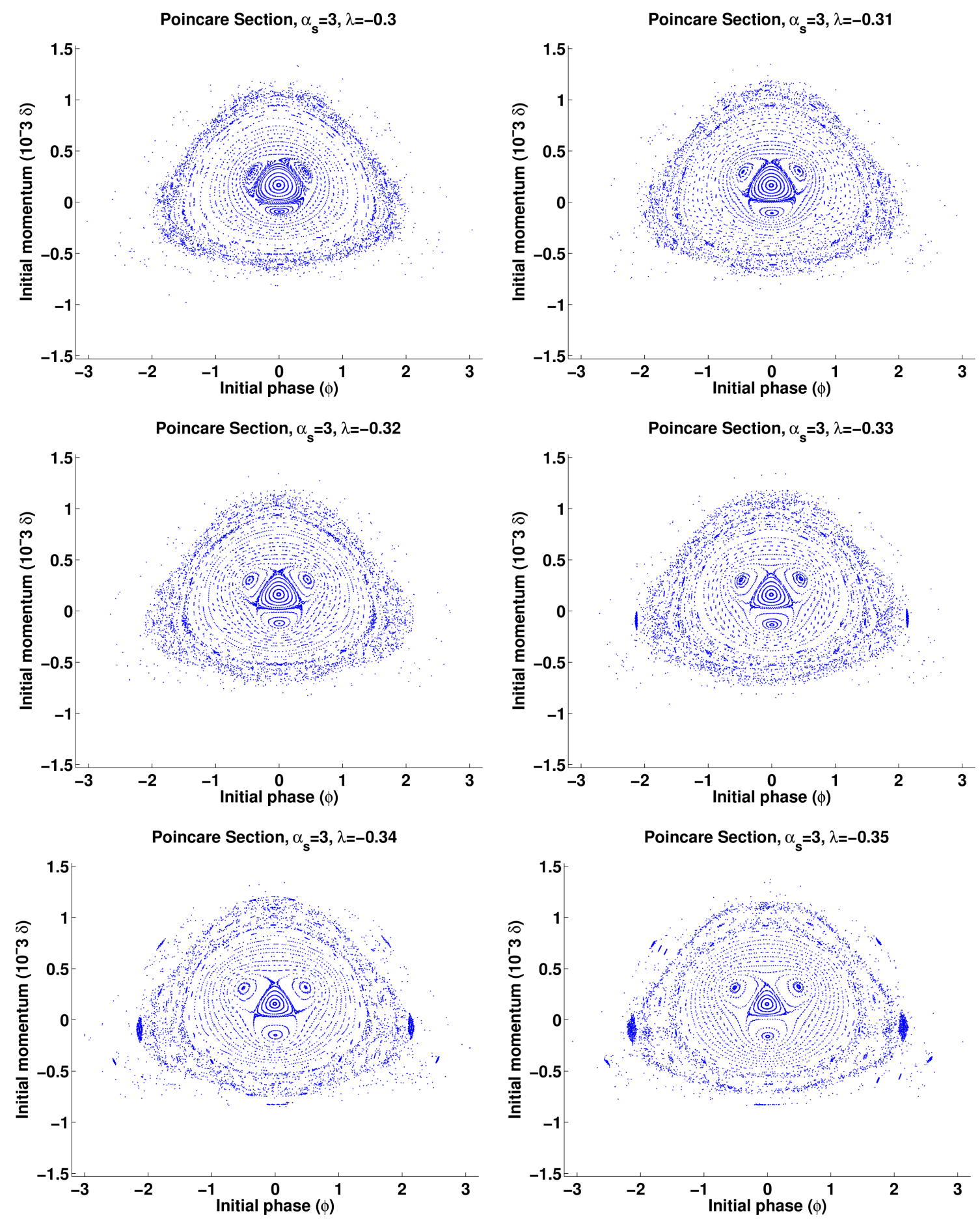

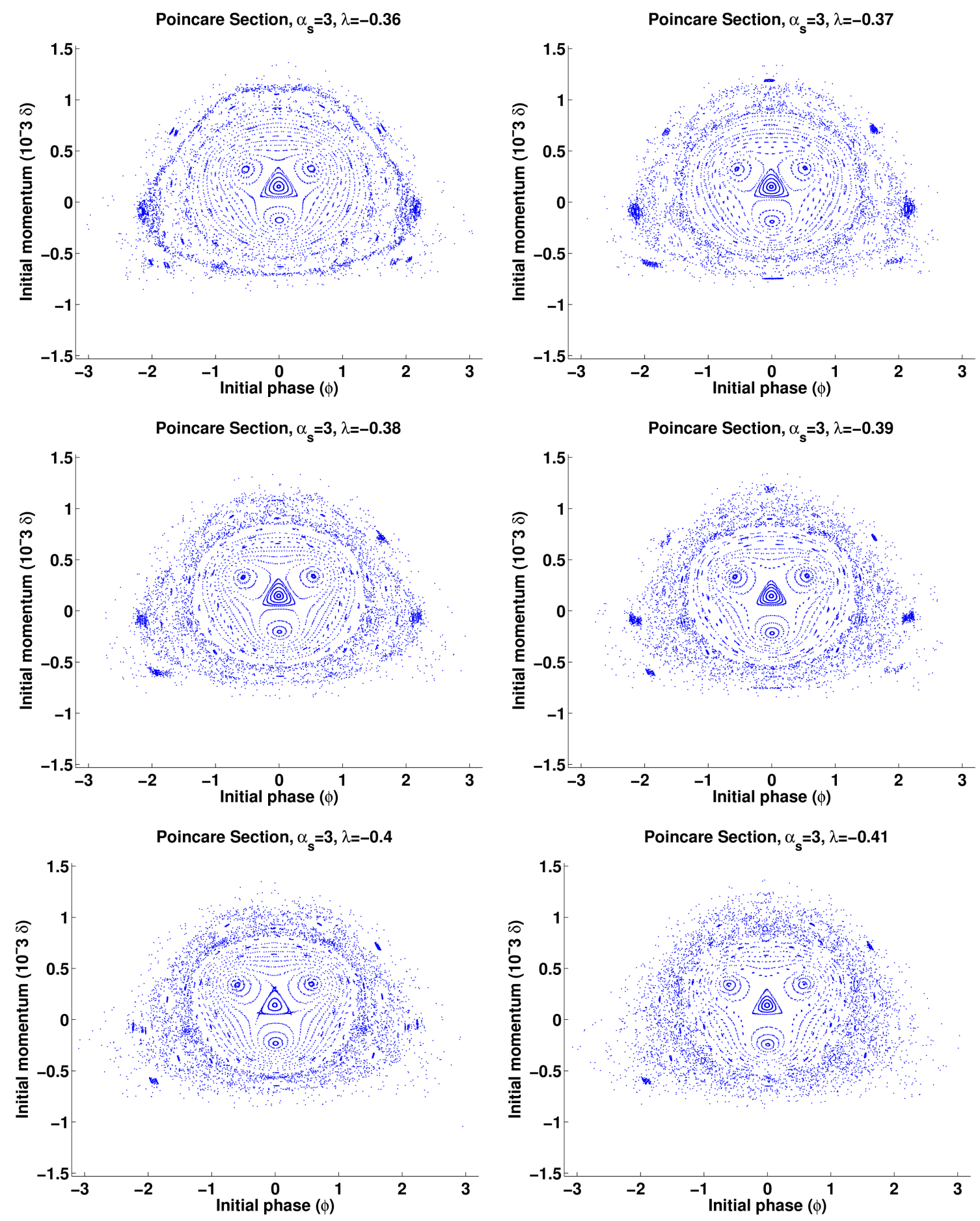

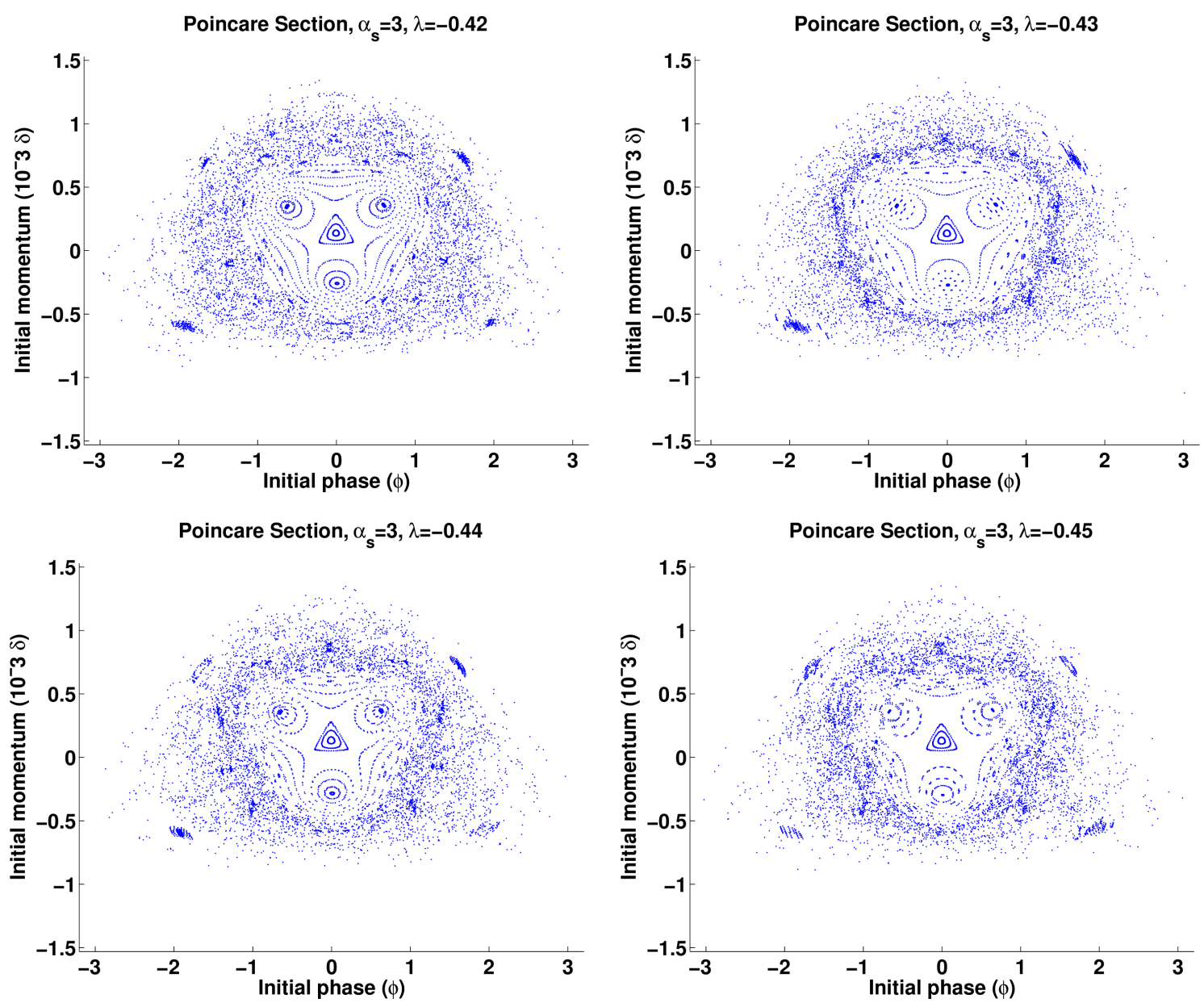
POINCARÉ MAPS, $\alpha_{s}=4$
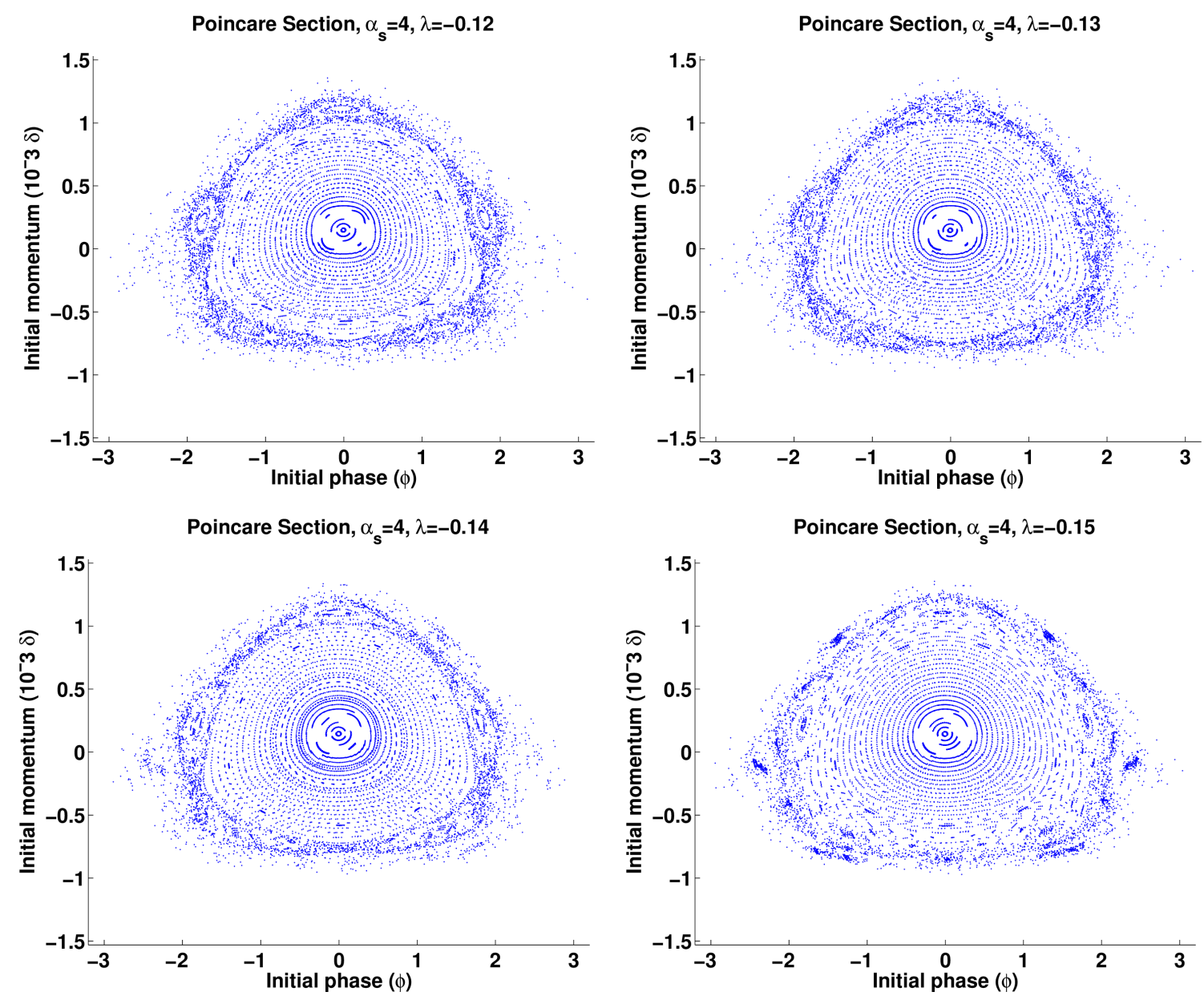

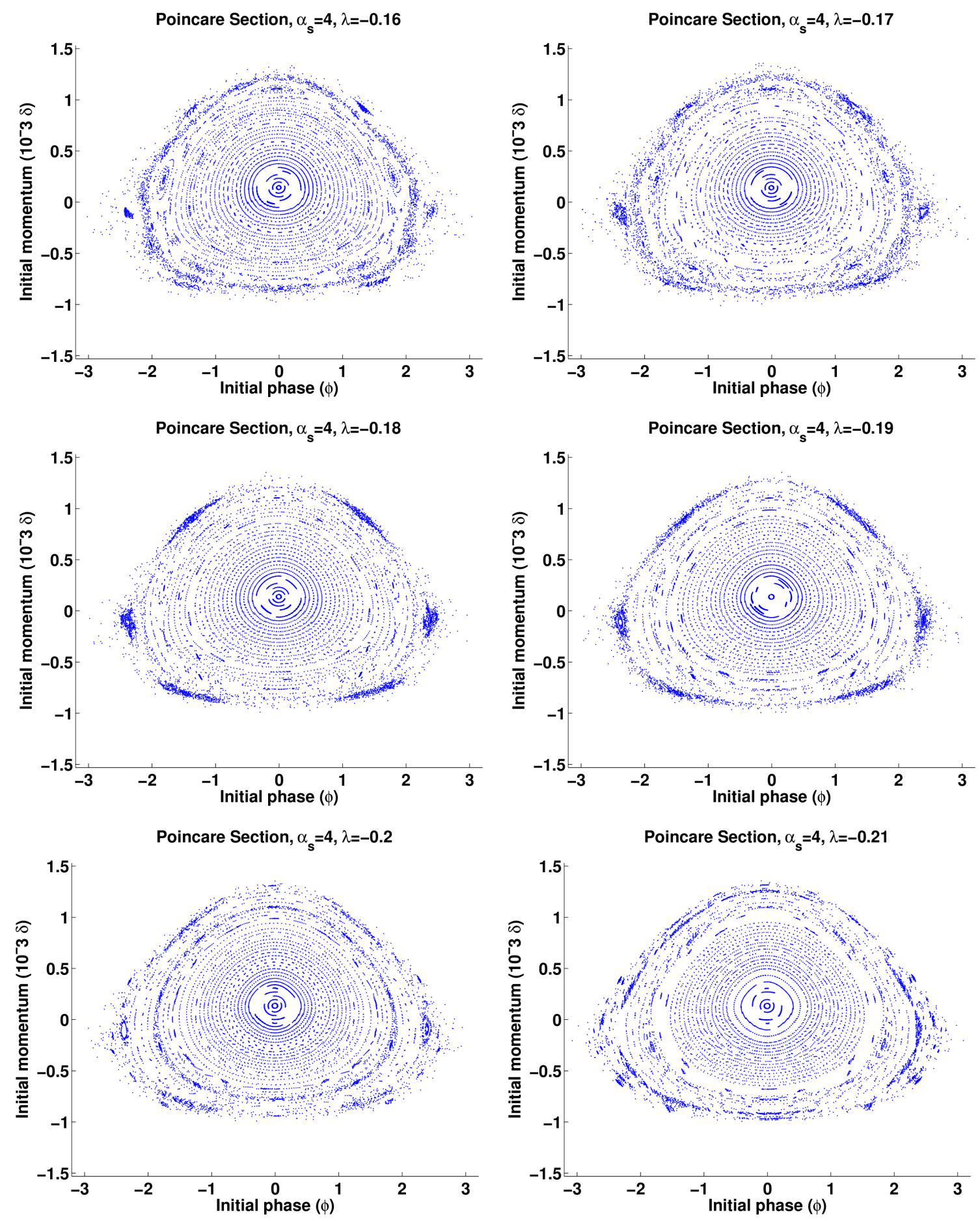

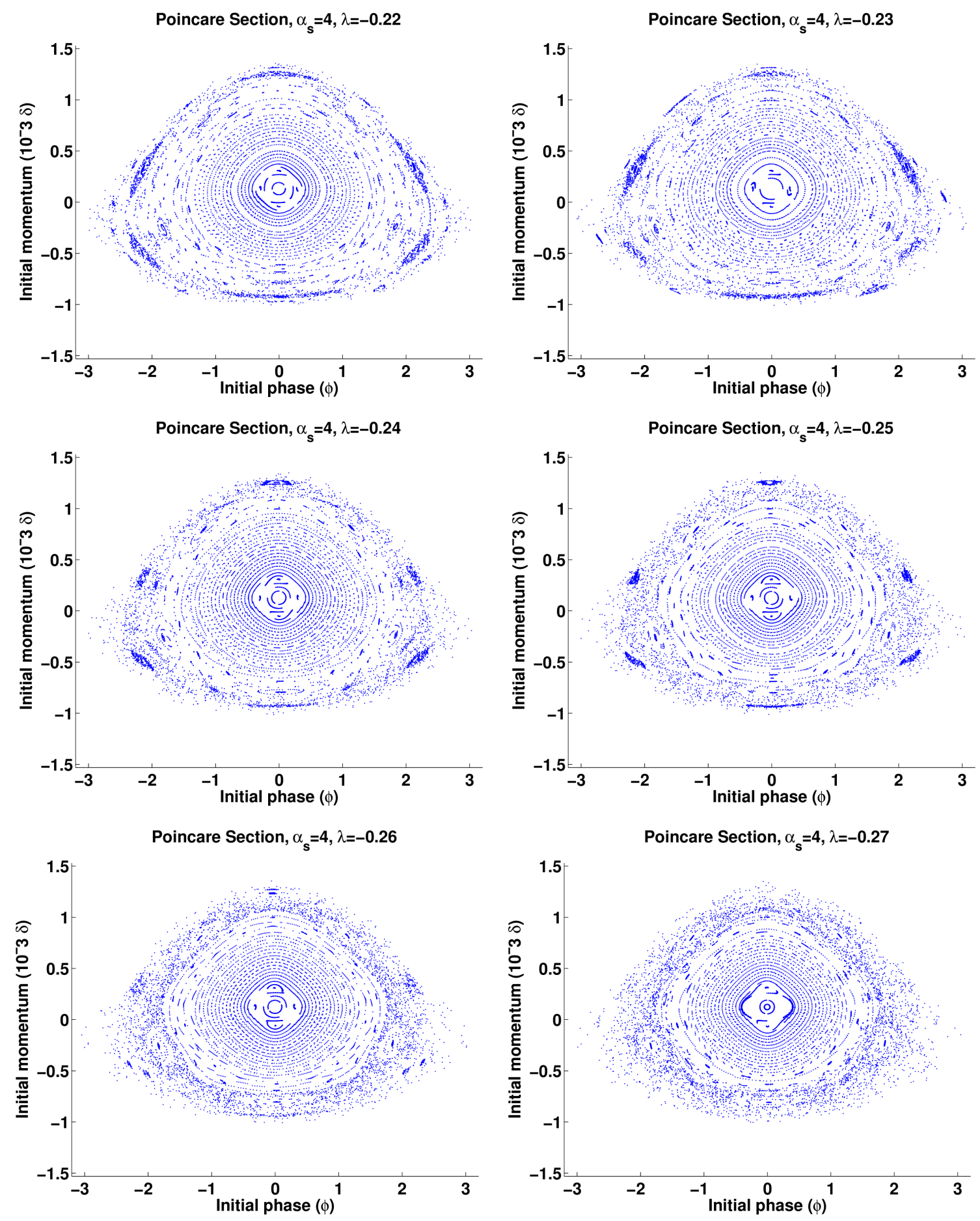

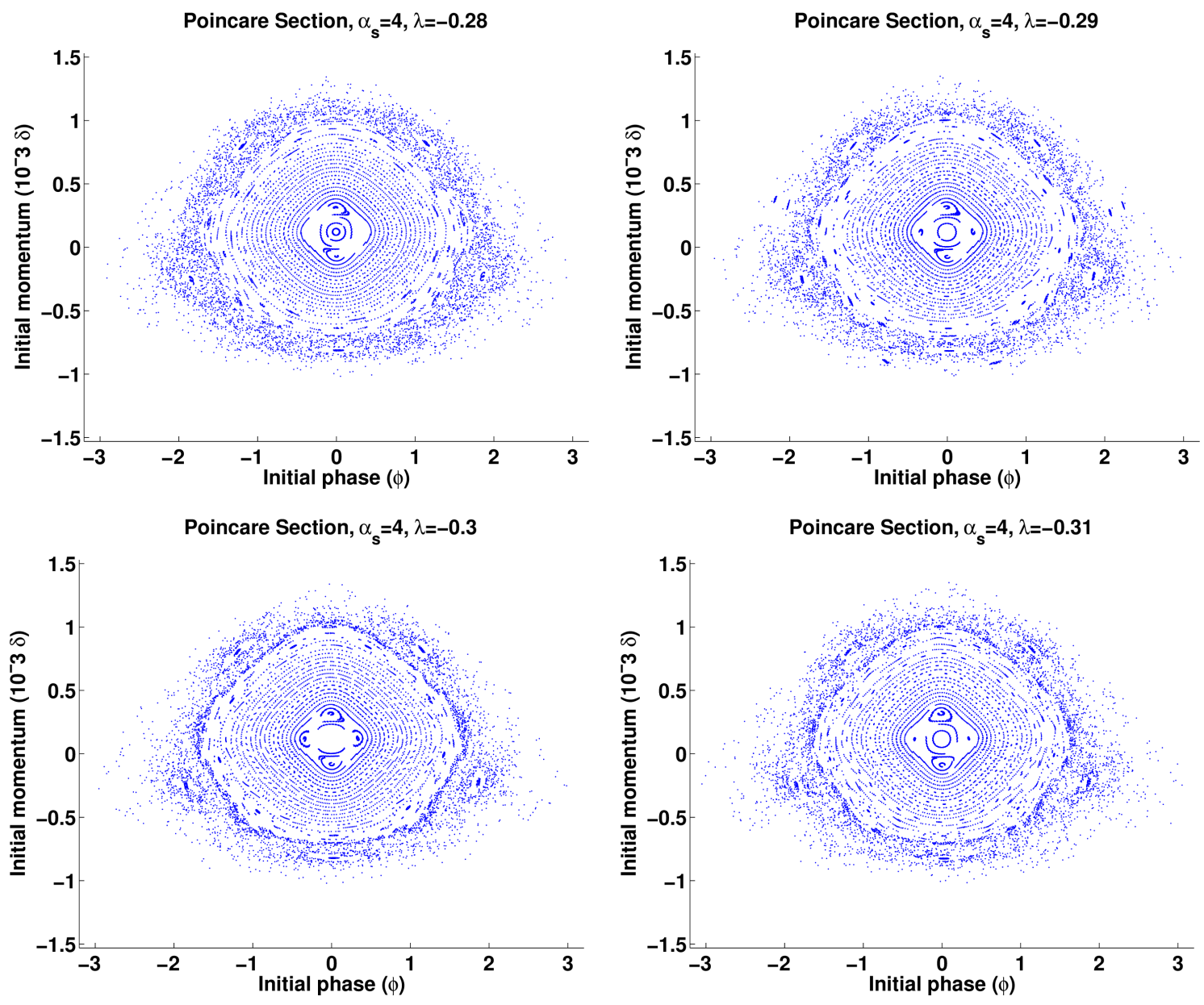
POINCARÉ MAPS, $\alpha_{s}=5$
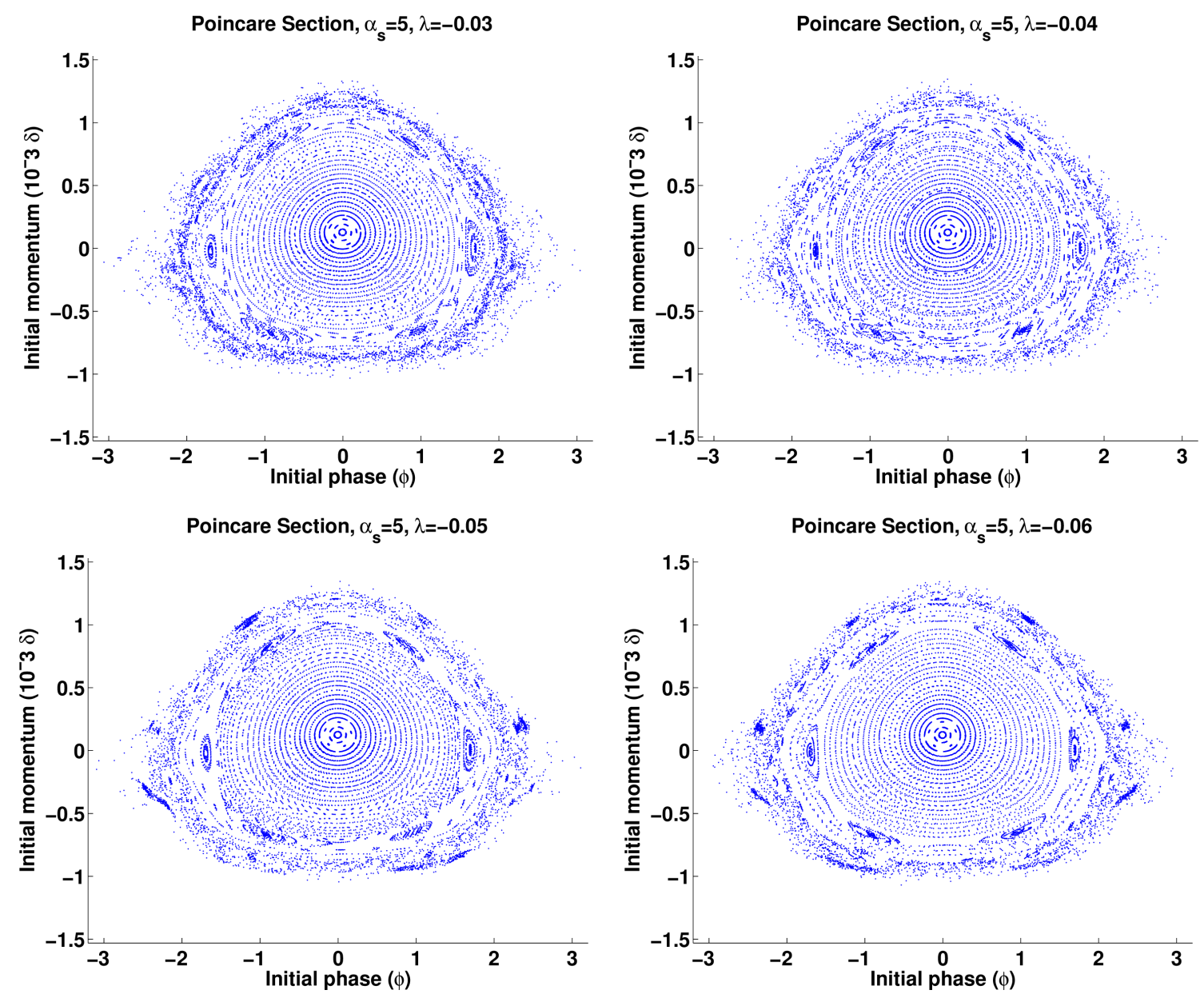

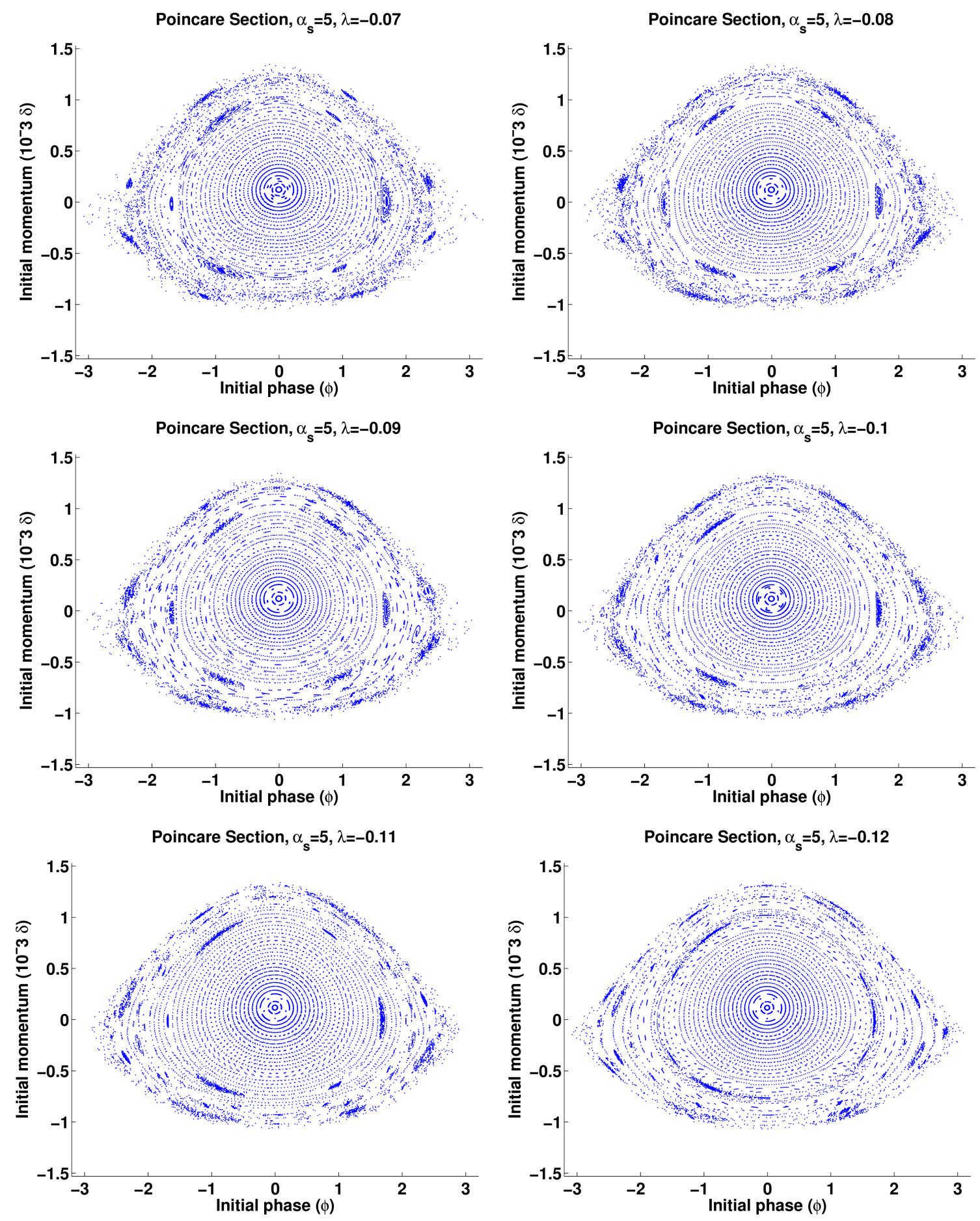

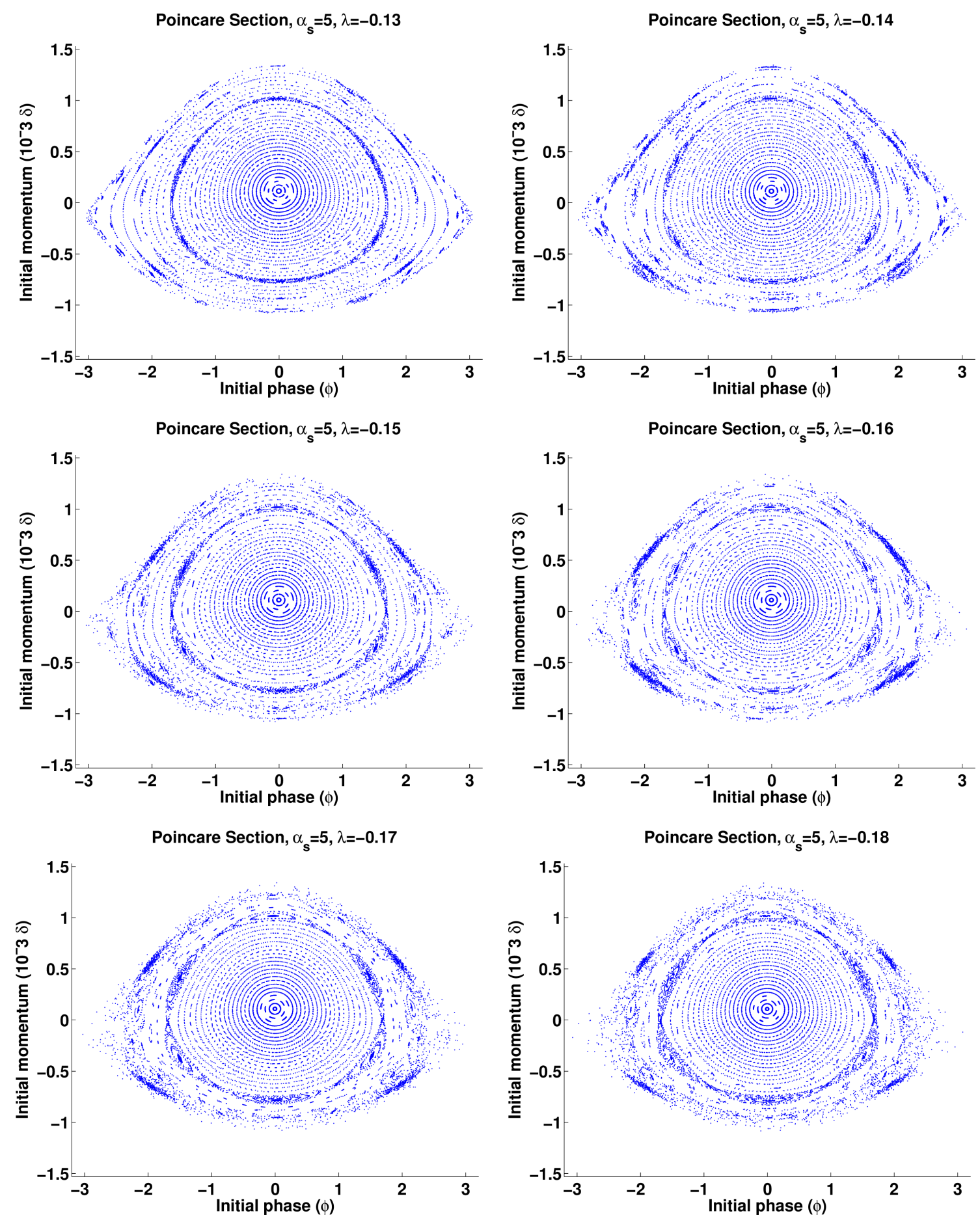

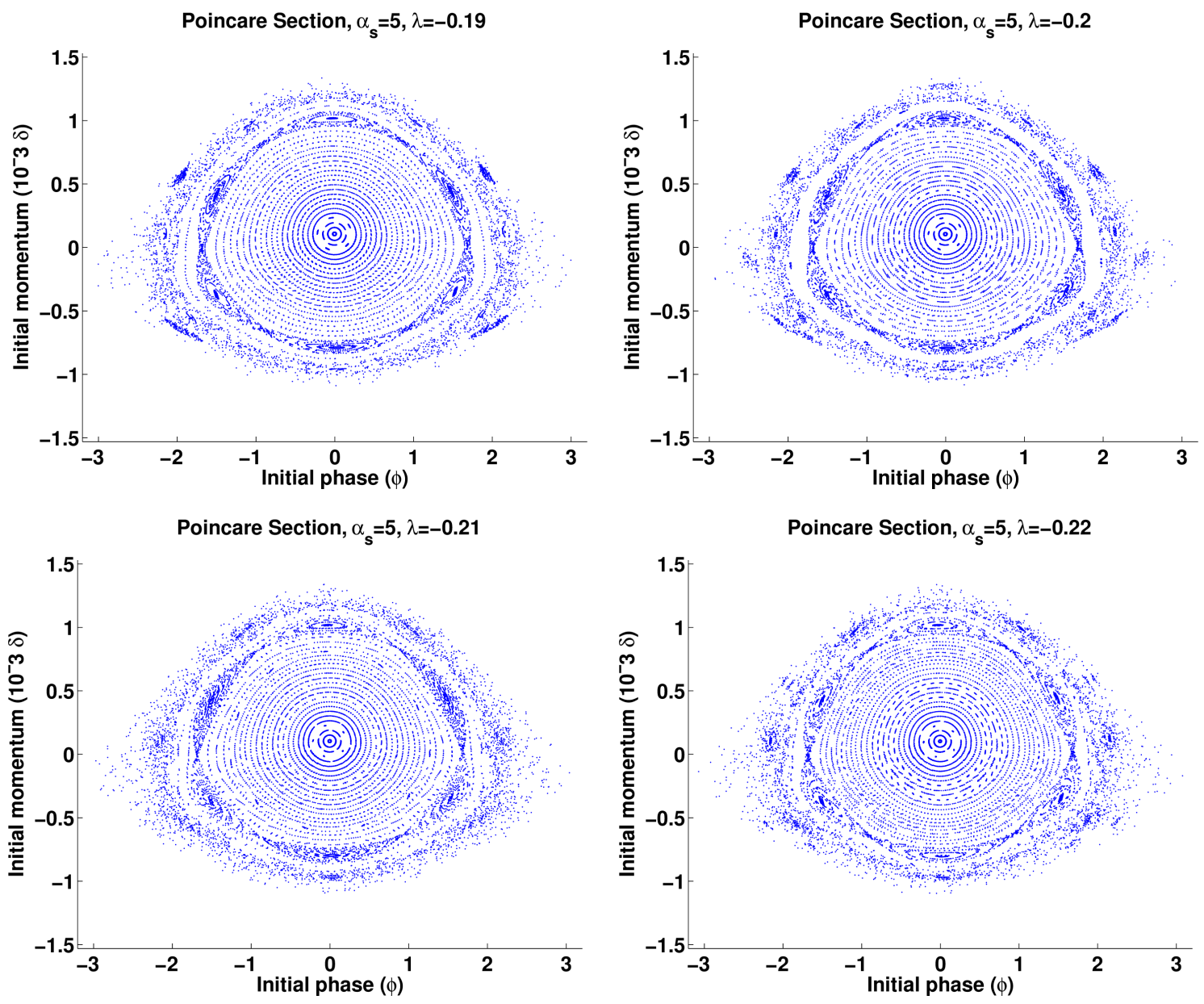
POINCARÉ MAPS, $\alpha_{s}=6$
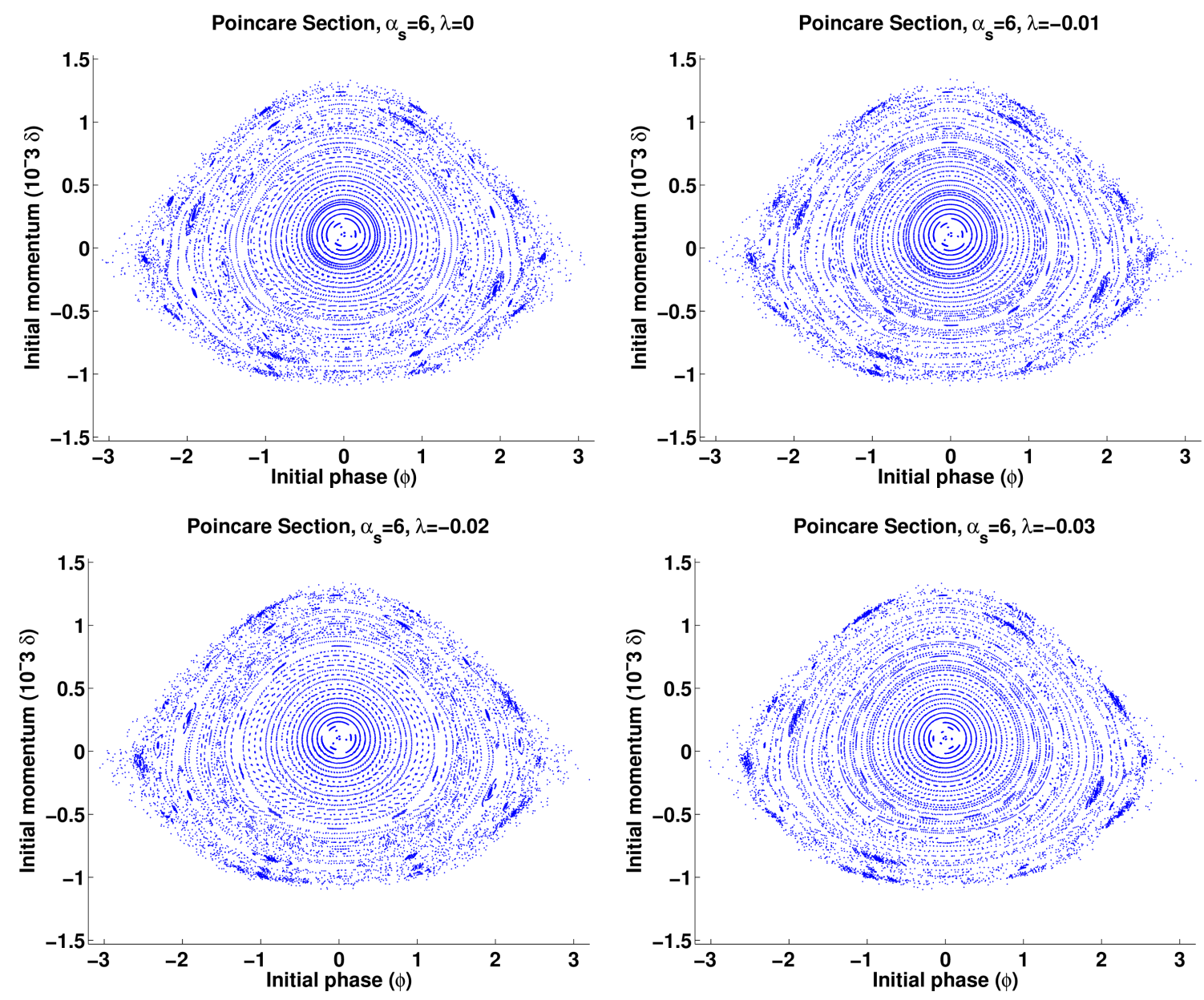

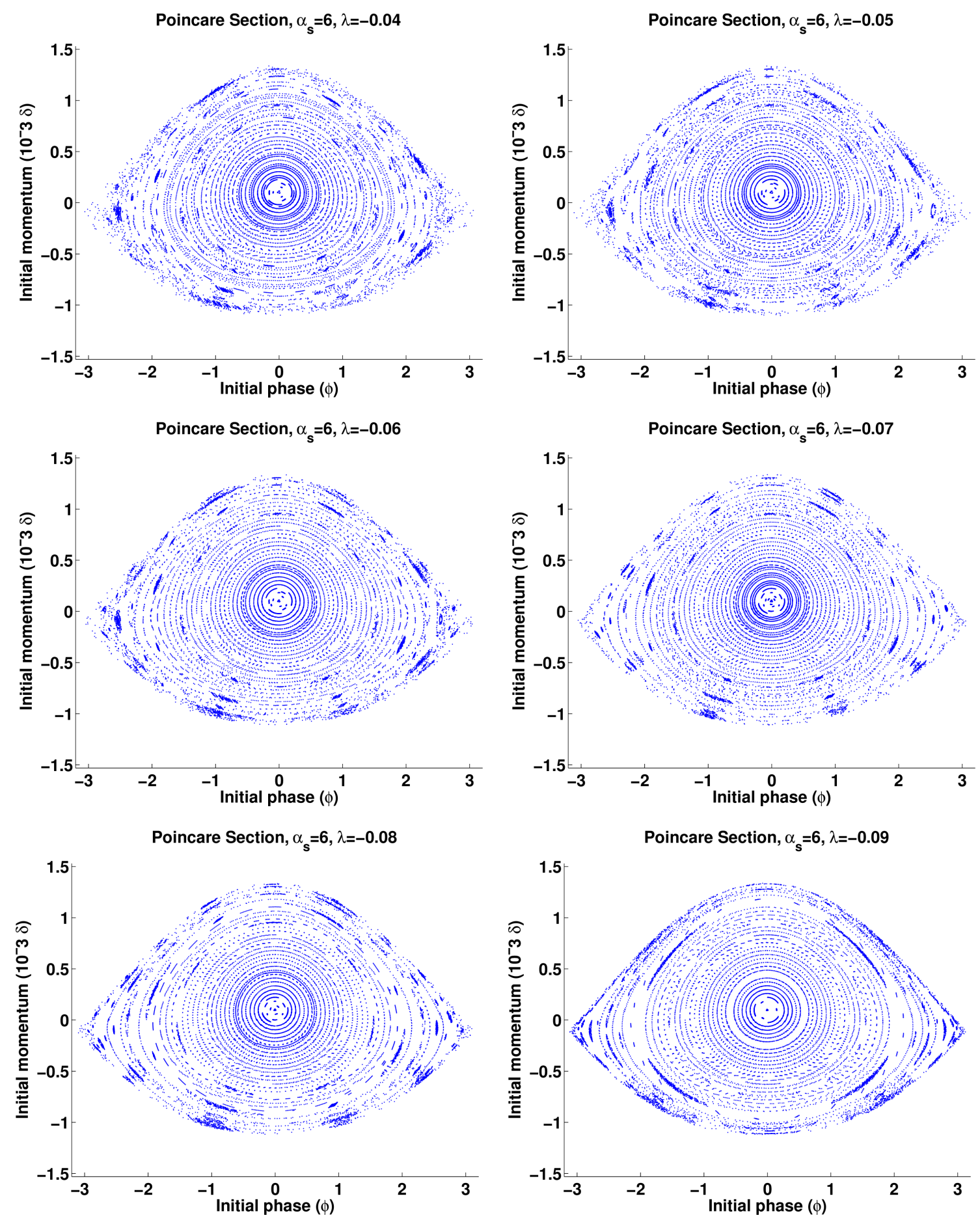

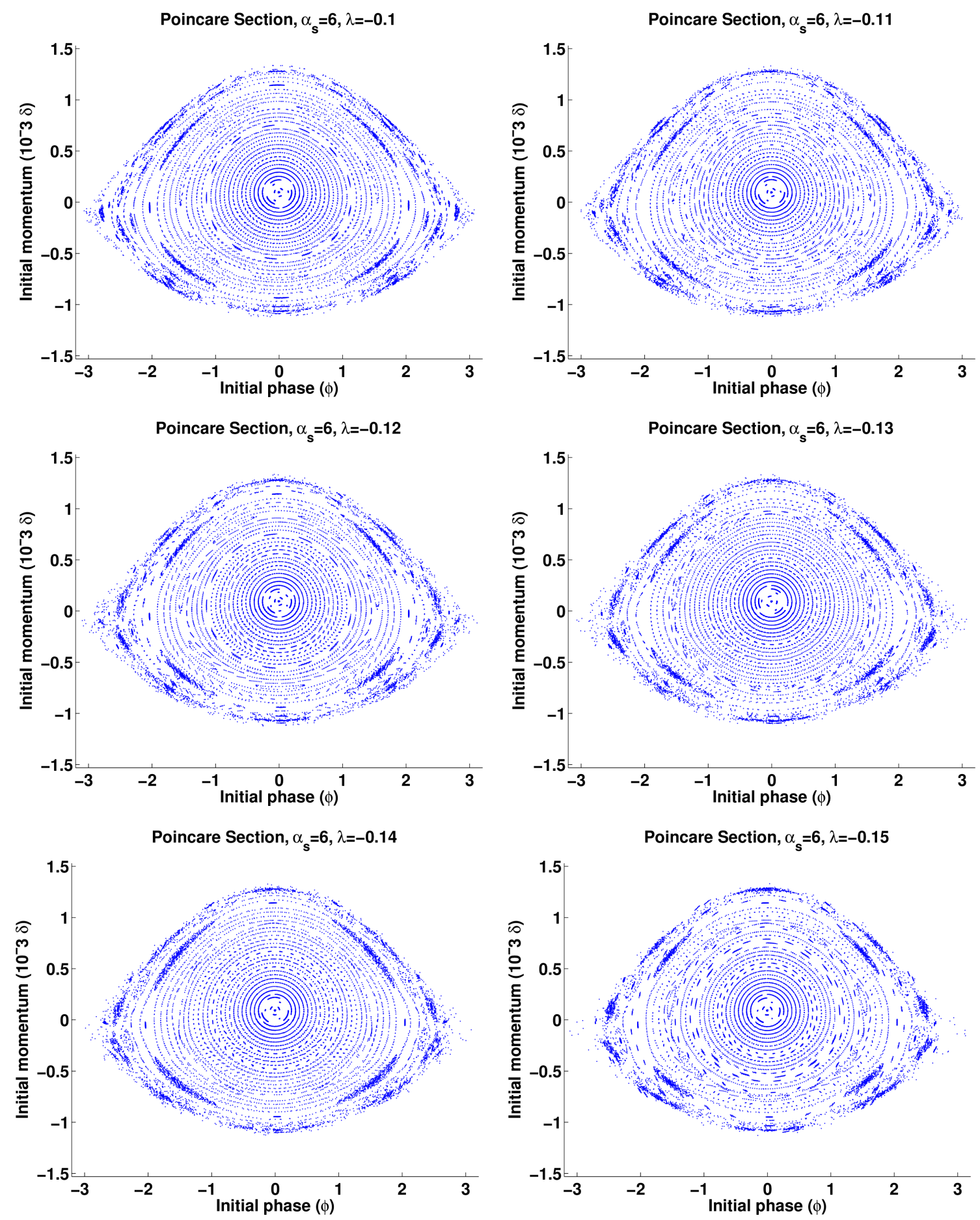

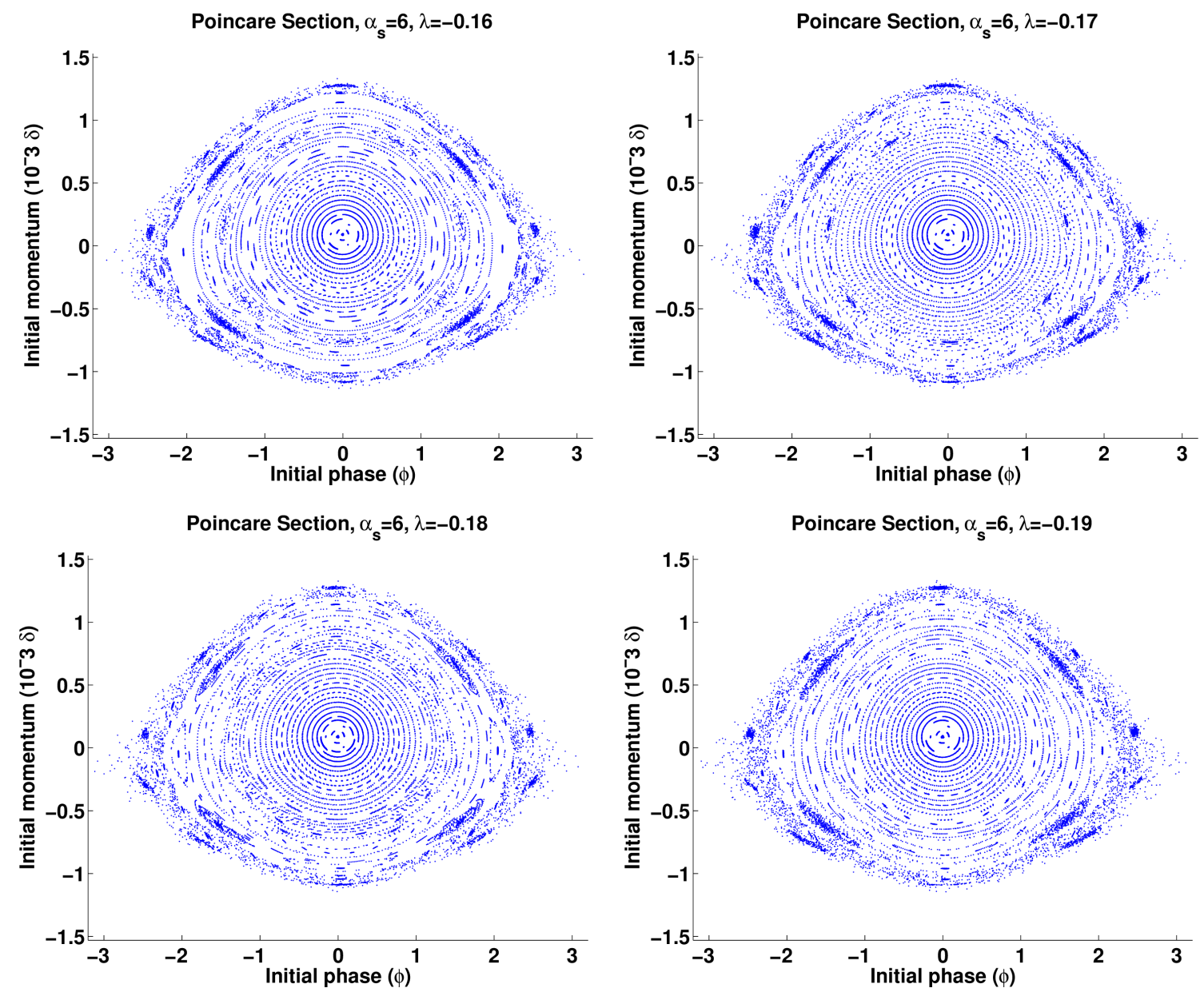


\section{BIBLIOGRAPHY}

[1] D. Barak, B. Harrison, and A. Watts, Fermilab Concepts Rookie Book, December 2013. http://operations.fnal.gov/rookie_books/concepts.pdf

[2] S. Y. Lee, Accelerator Physics, 3rd Ed. (World Scientific, Singapore, 2012).

[3] A. J. Lichtenberg and M. A. Lieberman, Regular and Chaotic Dynamics, 2nd Ed. (Springer, California, 1992).

[4] V. Shiltsev, S. Henderson, P. Hurh, I. Kourbanis, and V. Lebedev, Proc. PAC2013. http://accelconf.web.cern.ch/accelconf/pac2013/papers/mopac16.pdf

[5] B. C. Brown, P. Adamson, D. Capista, W. Chou, I. Kourbanis, D. K Morris, K. Seiya, G. H. Wu, and M. Yang, Phys. Rev. ST Accel. Beams 16, 071001 (2013). http: //journals.aps.org/prstab/abstract/10.1103/PhysRevSTAB.16.071001

[6] K. Seiya, Proc. HB2014. http://inspirehep.net/record/1362648/files/ tuo3lr02.pdf

[7] P. Derwent, S. Holmes, J. Hunt, A. Klebaner, I. Kourbanis, V. Lebedev, S. Mishra, S. Nagaitsev, W. Pellico, K. Seiya, C. Y. Tan, J. C. Theilacker, and R. M. Zwaska, Proton Improvement Plan-II, December 2013. http: //projectx-docdb.fnal.gov/cgi-bin/RetrieveFile?docid=1232\&filename= 1. $2 \% 20 \mathrm{MW} \% 20$ Report_Rev5.pdf\&version=3

[8] A. A. Aguilar-Arevalo et al. (MiniBooNE Collaboration), Phys. Rev. Lett. 98, 231801 (2007). http://journals.aps.org/prl/abstract/10.1103/PhysRevLett. 98.231801

[9] Fermilab Mu2e Conceptual Design Report, 2012. http://mu2e-docdb.fnal.gov/ cgi-bin/ShowDocument?docid=1169

[10] B. L. Roberts (New Muon (g-2) Collaboration), Chinese Physics C 34, 741 (2010). http://iopscience.iop.org/1674-1137/34/6/021

[11] P. Adamson et al. (MINOS Collaboration), Phys. Rev. Lett. 110, 251801 (2013).

[12] L. Fields et al. (MINERvA Collaboration), Phys. Rev. Lett. 111, 022501 (2013). G. A. Fiorentini et al. (MINERvA Collaboration), Phys. Rev. Lett. 111, 022502 (2013).

[13] L. Seuter (NOvA Collaboration), Fermilab Report No. NOVA-doc-14065-v1, 2015. http://nova-docdb.fnal.gov/cgi-bin/ShowDocument?docid=14065

[14] P. E. Reimer (SeaQuest Collaboration), Proc. SPIN10 projects-docdb.fnal.gov/ cgi-bin/RetrieveFile?docid=1225\&filename=Reimer_Spin2011.pdf

[15] P. Adamson, Proc. IPAC15. http://accelconf .web.cern.ch/AccelConf/IPAC2015/ papers/tuxb3.pdf 
[16] Fermilab Newsroom Press Release, July 8 2015. http://www.fnal.gov/pub/ presspass/press_releases/2015/Accelerator-World-Record-20150708.html

[17] Fermilab NOvA Technical Design Report, October 2007. http://inspirehep.net/ record/774999/files/fermilab-design-2007-01.pdf

[18] B. Worthel, R. Florian, C. Gattuso, E. McCrory, D. Patterson, C. Schmidt, T. Sullivan, and L. Wahl, Fermilab Linac Rookie Book, March 2012. http://operations.fnal. gov/rookie_books/LINAC_RB_v2_3.pdf

[19] J. S. Schmidt, B. Koubek, A. Schempp, C. Y. Tan, D. S. Bollinger, K. L. Duel, P. R. Karns, W. A. Pellico, V. E. Scarpine, B. A. Schupbach, and S. S. Kurennoy, Phys. Rev. ST Accel. Beams 17, 030102 (2014). http://journals.aps.org/prstab/ pdf/10.1103/PhysRevSTAB.17.030102

[20] V. E. Scarpine, C. Y. Tan, P. R. Karns, D. S. Bollinger, K. L. Duel, N. Eddy, N. Liu, A. Semenov, R. E. Tomlin, and W. A. Pellico, Proc. HB2012. http://accelconf . web. cern.ch/AccelConf/HB2012/papers/tuo1c06.pdf

[21] D. S. Bollinger, Proc. NIBS2010. http://scitation.aip.org/content/aip/ proceeding/aipcp/10.1063/1.3637398

[22] D. S. Bollinger, Rev. Sci. Instrum. 85, 02B121 (2014). http://inspirehep.net/ record/1278777/files/fermilab-conf-13-334-ad.pdf

[23] C. Y. Tan, D. S. Bollinger, K. L. Duel, J. R. Lackey, W. A. Pellico, Proc. PAC10. http://arxiv.org/ftp/arxiv/papers/1208/1208.3223.pdf

[24] R. Webber, W. Pellico, V. Lebedev, and D. McGinnis, Fermilab Report No. Beams-doc3781-v2, 2011. http://beamdocs.fnal.gov/AD/DocDB/0037/003781/002/The_Plan_ $\mathrm{v} 8 \cdot \mathrm{pdf}$

[25] B. Worthel, C. Broy, S. Chaurize, J. Crawford, B. Drendel, C. Gattuso, A. Meyhoefer, J. Morgan, D. Newhart, W. Pellico, J. Reyna, and T. Sullivan, Fermilab Booster Rookie Book, April 2009. http://operations.fnal.gov/rookie_books/Booster_V4.1.pdf

[26] C. M. Bhat, Proc. IPAC15. http://accelconf.web.cern.ch/AccelConf/IPAC2015/ papers/thpf $112 \cdot \mathrm{pdf}$

[27] X. Huang, S. Y. Lee, K. Y. Ng, and Y. Su, Phys. Rev. ST Accel. Beams 9, 014202 (2006). http://journals.aps.org/prstab/pdf/10.1103/PhysRevSTAB.9.014202

[28] M. Awida, J. Reid, V. Yakovlev, V. Lebedev, T. Khabiboulline, and M. Champion, Proc. IPAC12. http://accelconf.web.cern.ch/accelconf/ipac2012/papers/ thppc030.pdf

[29] W. Pellico, K. Domann, F. G. Garcia, K. Gollwitzer, K. Seyia, and B. Zwaska Proc. IPAC14. http: //accelconf . web. cern.ch/accelconf/IPAC2014/papers/thpme075. pdf 
[30] G. Romanov, M. H. Awida, T. N. Khabiboulline, W. Pellico, C. Y. Tan, I. Terechkine, V. P. Yakovlev, and R. M. Zwaska, Proc. IPAC14. http://accelconf.web.cern.ch/ AccelConf/IPAC2014/papers/thpri061.pdf

[31] C. Y. Tan, J. Dey, R. L. Madrak, W. Pellico, G. Romanov, D. Sun, and I. Terechkine, Proc. IPAC15. https://jacowfs.jlab.org/conf/y15/ipac15/prepress/WEPTY037. PDF

[32] C. M. Bhat, J. Griffin, J. MacLachlan, M. Martens, K. Meisner, and K. Y. Ng, Phys. Rev. E 55, 1028 (1997). http://journals.aps.org/pre/pdf/10.1103/PhysRevE. 55.1028

[33] V. Lebedev, A. Burov, W. Pellico, and X. Yang, Proc. HB2006. https://accelconf. web.cern.ch/accelconf/abdwhb06/PAPERS/TUAX02.PDF

[34] X. Yang, A. I. Drozhdin, and W. Pellico, Proc. PAC07 (TUPAS042). https:// accelconf . web. cern.ch/accelconf/p07/PAPERS/TUPAS042.PDF

[35] X. Yang, A. I. Drozhdin, and W. Pellico, Proc. PAC07 (TUPAS040). http:// accelconf . web.cern. ch/AccelConf/p07/PAPERS/TUPAS040.PDF.

[36] L. A. Ahrens, J. Alessi, M. Blaskiewicz, J. M. Brennan, K. Brown, C. Gardner, J. W. Glenn, T. Roser, K. S. Smith, W. VanAsselt, S. Y. Zhang, Proc. PAC99. http://epaper.kek.jp/p99/PAPERS/FRAR3.PDF

[37] M. Ellison, M. Ball, B. Brabson, J. Budnick, D. D. Caussyn, A. W. Chao, J. Collins, S. A. Curtis, V. Derenchuk, S. Dutt, G. East, T. Ellison, D. Friesel, W. Gabella, B. Hamilton, H. Huang, W. P. Jones, W. Lamble, S. Y. Lee, D. Li, M. G. Minty, S. Nagaitsev, K. Y. Ng, X. Pei, G. Rondeau, T. Sloan, M. Syphers, S. Tepikian, Y. Yan, and P. L. Zhang, Phys. Rev. Lett. 70, 5 (1993). http://journals .aps.org/ $\mathrm{prl} / \mathrm{pdf} / 10.1103 /$ PhysRevLett.70.591

[38] Fermilab Recycler Ring Technical Design Report, November 1996. http:// inspirehep.net/record/428434/files/fermilab-tm-1991.PDF

[39] T. Asher and S. Baginski, Fermilab Main Injector Rookie Book, June 2003. http: //operations.fnal.gov/rookie_books/Main_Injector_v1.1.pdf

[40] M. Xiao and D. Johnson, Proc. ICAP2009. http://lss.fnal.gov/archive/2009/ conf/fermilab-conf-09-414-ad.pdf

[41] P. Karns, C. Gattuso, M. Hue, D. Broemmelsiek, M. Stauffer, C. Edstrom, and S. Baginski, Fermilab Recycler Rookie Book, August 2010. http://operations.fnal. gov/rookie_books/Recycler_RB_v1.42.pdf

[42] V. Balbekov, Fermilab Technical Memo Fermilab-TM-2372, January 2007. http:// inspirehep.net/record/743192/files/fermilab-tm-2372.PDF 
[43] P. Adamson, W. J. Ashmanskas, G. W. Foster, S. Hansen, A. Marchionni, D. Nicklaus, A. Semenov, D. Wildman, and H. Kang, Proc. PAC05. https://accelconf.web. cern.ch/accelconf/p05/PAPERS/MPPP015.PDF

[44] Y. Alexahin, A. Burov, E. Gianfelice-Wendt, V. A. Lebedev, and A. A. Valishev, Fermilab Report No. Beams-doc-4780, 2015. https://beamdocs.fnal.gov/AD-private/ DocDB/ShowDocument?docid $=4780$

[45] J. Eldred, P. Adamson, D. Capista, N. Eddy, I. Kourbanis, D. K. Morris, J. Thangaraj, M. J. Yang, R. Zwaska, and Y. Ji, Proc. HB2014. http://accelconf .web.cern.ch/ AccelConf/HB2014/papers/tho4lr04.pdf

[46] J. Eldred, M. Backfish, C. Y. Tan, and R. Zwaska, Proc. IPAC15. https://jacowfs . jlab.org/conf/y15/ipac15/prepress/MOPMA027.PDF

[47] V. Balbekov, Fermilab Technical Memo Fermilab-FN-1001-APC, June 2015. http: //arxiv.org/pdf/1506.08139.pdf

[48] M. Backfish, J. Eldred, C. Y. Tan, and R. Zwaska, Trans. Nuc. Sci. PP, 99 (2015). http: //ieeexplore. ieee.org/xpl/articleDetails.j.jp?arnumber $=7305834$

[49] Report of the Particle Physics Project Prioritization Panel, May 2014. http://science.energy.gov/ /media/hep/hepap/pdf/May\%202014/FINAL_P5_ Report_Interactive_060214.pdf

[50] S. Nagaitsev, Proc. HB2014, http://epaper.kek.jp/HB2014/talks/tuo4lr01_ talk.pdf

[51] Long-Baseline Neutrino Facility (LBNF) and Deep Underground Neutrino Experiment (DUNE) Conceptual Design Report https://web.fnal.gov/project/LBNF/Lists/ Papers\%20and\%20Reports/CD1R. aspx.

[52] T. Davenne, O. Caretta, C. Densham, M. Fitton, P. Loveridge, P. Hurh, R. Zwaska, J. Hylen, and V. Papadimitriou, Phys. Rev. ST Accel. Beams 18, 091003 (2015). http://journals.aps.org/prstab/pdf/10.1103/PhysRevSTAB.18.091003

[53] A. Liu, Ph.D. thesis, Indiana University, 2015. http://physics.indiana.edu/ $\sim$ shylee/info/Thesis_Liu_Ao.pdf

[54] A. Liu, D. Neuffer, and A. Bross, NIM A 801, 44 (2015). http: //www. sciencedirect. com/science/article/pii/S0168900215009547

[55] Y. Alexahin, D. J. Summers Proc. IPAC10, http://accelconf.web.cern.ch/ AccelConf/IPAC10/papers/mope085.pdf

[56] A. Burov, Fermilab Technical Memo Fermilab-TM-2125, September 2000. http:// lss.fnal.gov/archive/2000/tm/TM-2125.pdf

[57] V. Danilov and S. Nagaitsev, Phys. Rev. ST Accel. Beams 13, 084002 (2010). http: //journals.aps.org/prstab/pdf/10.1103/PhysRevSTAB.13.084002 
[58] S. Nagaitsev, A. Valishev, D. Shatilov, and V. Danilov, Proc. IPAC12. http:// accelconf . web.cern.ch/AccelConf/IPAC2012/papers/MOYCP01.PDF

[59] D. Boussard and Y. Mizumachi, IEEE Trans. Nucl. Sci. 26, 3623 (1979).

[60] J. A. MacLachlan, Fermi National Accelerator Laboratory Report No. 0711, 2001.

[61] K. Seiya, T. Berenc, B. Chase, W. Chou, J. Dey, P. Joireman, I. Kourbanis, J. Reid, and D. Wildman, Proc. HB2006. https://accelconf.web.cern.ch/accelconf/ abdwhb06/PAPERS/THAY02.PDF

[62] T. Aaltonen et al. (CDF and D0 Collaborations), Phys. Rev. D 86, 092003 (2012).

[63] P. Derwent, S. Holmes, V. Lebedev, Fermilab Report No. BEAMS-DOC-4662-v1, 2014. https://beamdocs.fnal.gov/AD/DocDB/0046/004662/001/THIOA05_talk.pdf

[64] H. W. Broer, I. Hoveijn, M. van Noort, C. Simó, and G. Vegter, J. Dyn. Diff. Eq. 16, 897 (2004).

[65] X. Xu, M. Wiercigroch, and M. P. Cartmell, Chaos, Solitons Fractals 23, 1537 (2005).

[66] J. Eldred and R. Zwaska Phys. Rev. ST Accel. Beams 17, 094001 (2014).

[67] F. E. Mills, Brookhaven National Laboratory Report No. 15936, 1971.

[68] H. Zhang and T. W. Ma, Nonlinear Dyn. 70, 2433 (2012).

[69] S. Lenci, E. Pavlovskaia, G. Rega, and M. Wiercigroch, J. Sound Vib. 310, 243 (2008).

[70] E. I. Butikov, J. Phys. A 44, 295202 (2013).

[71] P. Hansen, K. A. Mitchell, J. B. Delos, Phys. Rev. E 73, 066226 (2006). http:// journals.aps.org/pre/pdf/10.1103/PhysRevE.73.066226

[72] S. Wiggins, 1st Ed. Chaotic Transport in Dynamical Systems, (Springer-Verlag, New York, United States, 1992).

[73] K. Seiya, T. Berenc, J. Dey, B. Chase, I. Kourbanis, J. MacLachlan, K. Meisner, R. Pasquinelli, J. Reid, C. Rivetta, and J. Steimel, Proc. PAC05. http://epaper. kek.jp/p05/PAPERS/MOPA004.PDF

[74] J. Dey, K. Koba, I. Kourbanis, and J. Reid, Proc. PAC01. https://www. overleaf . com/3045606czntrw\#/10545447/

[75] J. Dey and I. Kourbanis, Proc. PAC05. http://epaper.kek.jp/p05/PAPERS/ TPPT027.PDF

[76] R. Madrak and D. Wildman, Proc. PAC13. http://accelconf.web.cern.ch/ AccelConf/PAC2013/papers/wepma13.pdf

[77] I. Kourbanis, Fermilab Report No. BEAMS-DOC-4606-v1, 2014. https://beamdocs . fnal.gov/AD/DocDB/0046/004606/001/Doubling_MI_Power_20140520_IK.pdf 
[78] M. Xiao, Proc. IPAC12. http://accelconf.web.cern.ch/AccelConf/IPAC2012/ papers/TUPPR085.PDF

[79] N. J. Evans, Ph.D. thesis, University of Texas at Austin, 2014. https:// repositories.lib.utexas.edu/handle/2152/26055

[80] K. Seiya, B. Chase, J. Dey, P. Joireman, I. Kourbanis, and J. Reid, Proc. BEAM07. https://care-hhh.web.cern.ch/care-hhh/BEAM07/Proceedings/ Proceedings/Session\%205/S15-Seiya-letter.pdf

[81] J. Crisp, B. Fellenz, D. Heikkinen, M. A. Ibrahim, T. Meyer, and G. Vogel, J. Instrum. 7, T01002 (2012). iopscience.iop.org/article/10.1088/1748-0221/7/01/T01002

[82] J. Patrick, Fermilab Report No. Beams-doc-1762, 2005. https://beamdocs.fnal.gov/ AD/DocDB/0017/001762/001/smtf_patrick.ppt

[83] K. Hanke, H. Damerau, A. Deleu, A. Funken, R. Garoby, S. S. Gilardoni, N. Gilbert, B. Goddard, E. B. Holzer, A. M. Lombardi, D. Manglunki, M. Meddahi, B. Mikulec, E. Shaposhnikova, and M. Vretenar Proc. IPAC14. http://accelconf.web.cern.ch/ accelconf/IPAC2014/papers/thpme070.pdf

[84] T. Argyropoulos, T. Bohl and E. Shaposhnikova, LIU Day Talk, April 11 2014. https://indico.cern.ch/event/299470/session/3/contribution/16/ attachments/564153/777111/LIUSlipstacking_2014.pptx

[85] D. Valuch, Proc. Radioelektronika 2009. http://dvaluch.web.cern.ch/dvaluch/ seminar/radio $\% 20$ frequency $\% 20$ systems $\% 20$ of $\% 20$ the $\% 20$ cern $\% 20$ accelerators . pdf

[86] T. Mastoridis, P. Baudrenghien, C. Rivetta, J. D. Fox LARP Collaboration Meeting, May 8th 2012. https://indico.fnal.gov/getFile.py/access?contribId=28\& sessionId=3\&resId=0\&material Id=slides\&conf $I d=5072$

[87] J. Jose and E. Saletan, 1st Ed. Classical Dynamics: A Contemporary Approach, (Cambridge University Press, Cambridge, United Kingdom, 1998).

[88] J. Guckenheimer and P. Holmes, 6th Ed. Nonlinear Oscillations, Dynamical Systems, and Bifurcations of Vector Field, (Springer-Verlag, New York, United States, 2002). 


\section{Jeffrey Eldred}

jseldred@fnal.gov

\section{Education}

Indiana University, Bloomington, IN

PhD in Physics, August 2011 to December 2015

Fermilab Joint PhD program, June 2013 to December 2015

College of William \& Mary, Williamsburg, VA

BS, Physics and Applied Mathematics, August 2007 to May 2011

Magna cum laude with departmental honors in physics

\section{Notable Honors \& Achievements}

- Received IEEE NPSS Graduate Scholarship Award (2015).

- Excellent performance on qualifying exams (2012).

- Elected Graduate Student President, Indiana University Department of Physics (2012).

- Received additional award of \$5,000 upon enrollment in Indiana University Physics PhD program (2011)

- Named Monroe Scholar, received grant for independent research, College of William \& Mary (2007-2011).

\section{Publications}

PRST-AB (Anticipated): J. Eldred and R. Zwaska. Proposed Cavity for Enhanced Dynamical Stability of Slip-stacking. Submitting to Physical Review Special Topics - Accelerators and Beams.

TNS: M. Backfish, J. Eldred, C.Y. Tan and R. Zwaska. Test of Beampipe Coatings for Electron Cloud Mitigation in Fermilab Main Injector. IEEE Transactions on Nuclear Science Special Issue, accepted for publication.

http://arxiv.org/pdf/1507.07281.pdf 
PRST-AB: J. Eldred and R. Zwaska. Dynamical Stability of Slip-stacking Particles. Physical Review Special Topics - Accelerators and Beams, published September 2014.

https://journals.aps.org/prstab/abstract/10.1103/PhysRevSTAB.17.094001

LAMA: J. Eldred, L. Rodman, and I. Spitkovsky. Numerical Ranges of Companion Matrices: flat portions on the boundary. Linear and Multilinear Algebra, published November 2011.

http://www.tandfonline.com/doi/abs/10.1080/03081087.2011.634415

Conference Papers and Presentations

Conference Paper, Winning Poster: J. Eldred, M. Backfish, C.Y. Tan and R. Zwaska. Electron Cloud Measurements in Fermilab Main Injector and Recycler. International Particle Accelerator Conference (IPAC15), May 2015. 2nd Place 2015 Fermilab User's Meeting Graduate and Postdoc Poster Competition.

https://jacowfs.jlab.org/conf/y15/ipac15/prepress/MOPMA027.PDF

Invited Seminar: J. Eldred. Dynamics Problems in Particle Accelerators and Pendulums. Floris Takens Seminar, University of Groningen, The Netherlands, April 2015.

Contributed Talk, Paper: J. Eldred, P. Adamson, D. Capista, N. Eddy, I. Kourbanis, D.K. Morris, J. Thangaraj, M.J. Yang, and R. Zwaska. Fast Transverse Instability and Electron Cloud Measurements in Fermilab Recycler. 54th ICFA Advanced Beam Dynamics Workshop (HB2014), November 2014.

http://accelconf. web. cern. ch/AccelConf/HB2014/papers/tho4lr04.pdf

Conference Paper: J. Eldred and R. Zwaska. Slip-stacking Dynamics and the $20 \mathrm{~Hz}$ Booster. 54th ICFA Advanced Beam Dynamics Workshop (HB2014), November 2014. http://accelconf . web.cern.ch/AccelConf/HB2014/papers/mopab12.pdf 
Winning Poster: J. Eldred and R. Zwaska. Dynamical Stability of Slip-stacking Particles. 1st Place 2014 Fermilab User's Meeting Graduate and Postdoc Poster Competition, June 2014.

Technical Memo: J. Eldred and R. Zwaska. Implications of 20-Hz Booster cycle-rate for slipstacking. FERMILAB-TM-2587-APC, June 2014.

https://inspirehep.net/record/1303257

Conference Paper: D. Douglas, K. Beard, J. Eldred, P. Evtushenko, A. Jenkins, W. Moore, L. Osborne, D. Sexton, and C. Tennant. Simplified Charged Particle Beam Transport Modeling using Commonly Available Commercial Software. Particle Accelerator Conference (PAC07), June 2007.

http://accelconf . web.cern. ch/AccelConf/p07/PAPERS/THPAS073.PDF

\section{Postdoctoral Plans}

1. Postdoc Researcher, Fermilab IOTA Collaboration, January 2016 - January 2019

Accelerator Physics, Dynamics, Data Analysis, and Electronics with Alexander Valishev, Fermilab Staff Scientist

- Determine the feasibility of integrable optics and electron lens space-charge compensation for a high-power RCS.

- Full analytical, computational and technical evaluation of a scenario for multi-MW neutrino facility based on an innovative high intensity RCS.

\section{Associate Instructor, "CM and E\&M," USPAS, January 25 - February 52016}

Physics Education, Accelerator Physics

with Gregg Penn, Staff Scientist at LBL

- Graduate-level course to accelerator physics students and professionals. 


\section{Graduate Research \& Teaching}

\section{Graduate Student, Fermilab APC, May 2013 - December 2015}

Accelerator Physics, Dynamics, Data Analysis, and Electronics

with Robert Zwaska, Fermilab Staff Scientist, and Shyh-Yuan Lee, IU Professor of Physics

- Experimental, analytic, and numerical analysis of slip-stacking dynamics.

- Measurement, data acquisition, and analysis for several electron-cloud experiments.

- Diagnosis of a new high-intensity Recycler instability using stripline and ecloud measurements.

\section{Co-Instructor, "MATLAB for Physics," USPAS, June 16th - 20th 2014}

Physics Education, Accelerator Physics, and Programming

co-taught with Daniel R. Green, Scientist Emeritus and author

- Graduate-level course to accelerator physics students and professionals.

- Taught MATLAB, classic physics problems, and core accelerator physics content.

- Received exceptionally positive course evaluations.

\section{Graduate Student, Indiana University CEEM, May 2012 - May 2013}

Accelerator Physics and Data Science

with Shyh-Yuan Lee, Professor of Physics at Indiana University

- Collected first betatron and synchrotron oscillation data for ALPHA electron ring.

- Completed a lattice design for a Compact Medical Synchrotron.

- Innovative treatment of Independent Component Analysis (ICA) algorithms.

6. Associate Instructor, Intro Physics Lab, Indiana University, August 2011 - May 2012 Physics Education 
7. VMEC Program, BAE Systems Semiconductor Technology Center, May 2011 - August 2011

Quantitative Analysis and Semiconductor Engineering with Stewart Ocheltree and Craig Hill at the BAE STC in Manassas, VA

- Quality assurance testing and statistical analysis on etching and ashing machines.

8. Monroe Scholar Grant for Mathematics Research, November 2009 - June 2011

Linear Algebra and Quantum Information with Ilya Spitkovsky, Professor of Mathematics at William \& Mary

- Published work characterizing the numerical range of companion matrices.

\section{Undergraduate Research}

9. NOvA Experiment at Fermilab, May 2010 - August 2010

Neutrino Physics, Detector Physics, and Data Analysis

under Patricia Vahle, Assistant Professor of Physics at William \& Mary

10. REU in Atomic-Optical Lab at William \& Mary, May 2009 - August 2009

Atomic-Optical Physics, Electronics, and Quantum Information

under Irina Novikova, Assistant Professor of Physics at William \& Mary

11. SULI Program at Hall B Jefferson Lab, May 2008 - August 2008

Detector Physics and Electronics

under Stepan Stepanyan, Staff Scientist at Jefferson Lab Experimental Hall B

12. JLab Free Electron Laser at Jefferson Lab, October 2006 - July 2007

Accelerator Physics and Programming

under David Douglas, Staff Scientist at Jefferson Lab Free Electron Laser 